REPORT ON THE U.S. GEOLOGICAL SURVEY'S EVALUATION PROGRAM FOR STANDARD REFERENCE SAMPLES DISTRIBUTED IN APRIL 1994:

T-129 (TRACE CONSTITUENTS), M-130 (MAJOR CONSTITUENTS), N-42 (NUTRIENTS), P-22 (LOW IONIC STRENGTH), AND $\mathrm{Hg}-18$ (MERCURY)

by H. Keith Long and Jerry W. Farrar

U.S. GEOLOGICAL SURVEY

Open-File Report 94-369

Golden, Colorado

1994 


\section{DEPARTMENT OF INTERIOR \\ BRUCE BABBITT, Secretary}

US.S GEOLOGICAL SURVEY

Gordon P. Eaton, Director

For additional information write to:

William J. Shampine

U.S. Geological Survey

Water Resources Division, CR

Box 25046. Mail Stop 401

Denver Federal Center

Denver, CO 80225-0046
Copies of this report can be purchased from:

U.S. Geological Survey

Books and Open-File Reports

Federal Center, Bldg. 810

Box 25425

Denver, CO 80225-0425 


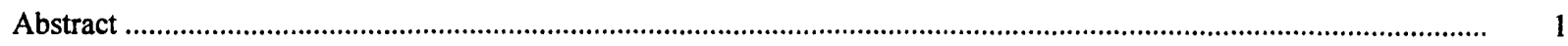

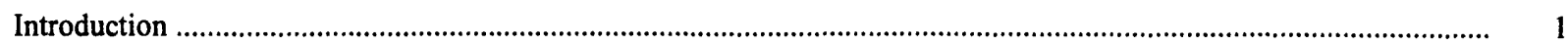

Purpose and scope

Preparation of standard reference water samples ..............................................................................................

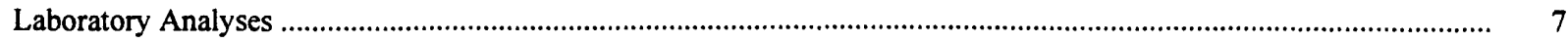

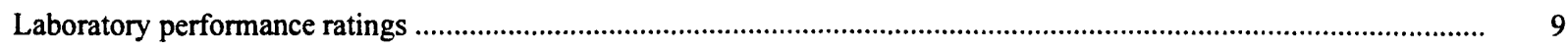

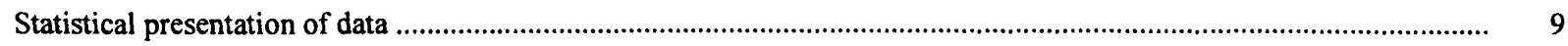

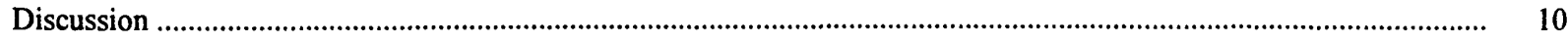

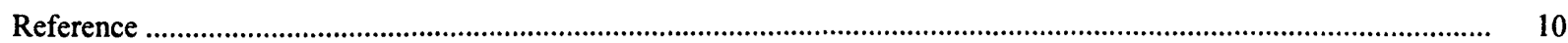

\section{FIGURE}

Figure 1. Statistical parameters shown on reported-data graphs

\section{TABLES}

Table 1. Laboratory participants in the analyses of standard reference samples distributed in April 1994 ................... 3

2. Analytes determined in standard reference samples distributed in April 1994........................................... 7

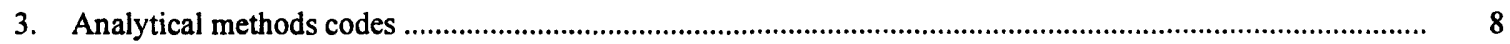

4. Overall laboratory performance ratings for standard reference water samples distributed in April $1994 \ldots \ldots . . . \quad 11$

5. Laboratory performance ratings for standard reference water sample $\mathrm{T}-129$ (trace constituents) ......................

6. Laboratory performance ratings for standard reference water sample $\mathrm{M}-130$ (major constituents) ................. 21

7. Laboratory performance ratings for standard reference water sample $\mathrm{N}-42$ (nutrients) .................................... 27

8. Laboratory performance ratings for standard reference water sample P-22 (low ionic strength) ...................... 29

9. Laboratory performance ratings for standard reference water sample $\mathrm{Hg}-18$ (mercury) ................................. 31

10. Statistical summary of reported data for standard reference water sample T-129 (trace constituents) ............... 32

11. Statistical summary of reported data for standard reference water sample $M-130$ (major constituents) ........... 59

12. Statistical summary of reported data for standard reference water sample $\mathrm{N}-42$ (nutrients) ............................. 76

13. Statistical summary of reported data for standard reference water sample P-22 (low ionic strength) ............... 87

14. Statistical summary of reported data for standard reference water sample Hg-18 (mercury) ............................ 99

15. Most probable values for constituents and properties in standard reference samples distributed in April 1994 


\title{
REPORT ON THE U.S. GEOLOGICAL SURVEY'S EVALUATION PROGRAM FOR STANDARD REFERENCE SAMPLES DISTRIBUTED IN APRIL 1994: T-129 (TRACE CONSTITUENTS), M-130 (MAJOR CONSTITUENTS), N-42 (NUTRIENTS), P-22 (LOW IONIC STRENGTH),AND Hg-18 (MERCURY)
}

\author{
By H. Keith Long and Jerry W. Farrar
}

\begin{abstract}
This report presents the results of the U.S. Geological Survey's analytical evaluation program for 5 standard reference samples--T-129 (trace constituents), M-130 (major constituents), N-42 (nutrients), P-22 (low ionic strength), and Hg-18 (mercury)--that were distributed in April 1994 to 157 laboratories registered in the U.S. Geological Survey sponsored interlaboratory testing program. Analytical data that were received from 133 of the laboratories were evaluated with respect to: overall laboratory performance and relative laboratory performance for each analyte in the five reference samples. Results of these evaluations are presented in tabular form. Also presented are tables and graphs summarizing the analytical data provided by each laboratory for each analyte in the five standard reference samples. The most probable value for each analyte was determined using nonparametric statistics.
\end{abstract}

\section{INTRODUCTION}

The U.S. Geological Survey (USGS) conducts an interlaboratory evaluation program semiannually. This program provides a variety of reference materials to accomplish quality assurance testing of laboratories and to provide an adequate supply of samples that contribute to quality control programs of participating laboratories. Natural-matrix reference materials are preferred for use in this interlaboratory evaluation program. A series of samples are prepared and distributed each spring and fall. Occasionally, sediment samples are provided.

The program began in 1962 with a single sample containing major constituents that was prepared from distilled water and reagent grade chemicals. Twenty-three USGS laboratories participated in the 1962 determinations of six analytes in the major standard reference sample (SRS). Since that time, objectives of the program have been to:

(1) evaluate and improve the performance of USGS and other participating laboratories;

(2) provide a library of carefully prepared, homogeneous, stable reference materials for use in the quality control programs of laboratories;

(3) identify analytical problem areas;

(4) identify quality assurance needs with respect to environmental analyses and develop new reference materials to meet these needs; and

(5) ascertain the accuracy and precision of analytical methods. 
One hundred eighty-five USGS and non-USGS laboratories are registered in the program, which can currently provide eight standard reference sample types:

1. Trace constituents.

2. Major constituents.

3. Nutrients.

4. Low ionic strength.

5. Mercury.

6. Whole water (water with suspended sediment).

7. Acid mine drainage.

8. Sediment (bed material) for major and trace constituents.

When sufficient data are available, a most probable value is statistically determined for each analyte in the SRS.

Though this is not a laboratory certification program, participation in this continuing quality assurance program is mandatory for all laboratories providing water-analyses data for USGS data storage or use (publications). Federal, State, municipal, and university laboratories can participate even though they do not provide data to the USGS. Analyses of these SRS provides the means to alert participating laboratories of possible deficiencies in their analytical operations, and also provides reference materials for in-house quality control programs. Participating laboratories are identifed only by a confidential code number.

A library of SRS, from previous evaluations, are available on request. Participating laboratories can request previous SRS for further testing, continuing quality assurance, and quality control programs by contacting:

Chief Laboratory Section, BQA

U.S. Geological Survey

Branch of Quality Assurance

Denver Federal Center

Box 25046 MS 401

Denver, CO 80225-0046

\section{Purpose and Scope}

This report summarizes the analytical results submitted by 133 (table 1) of the 157 laboratories that requested and were shipped SRS for the April 1994 evaluation. Not all SRS are requested, nor necessarily analyzed by all the laboratories; nor do all laboratories enrolled in the program participate in each evaluation. Analytical results for the following, which were mailed the week of April 25, 1994, are presented in this report:

$\begin{array}{ll}\text { T-129 } & \text { Trace constituents } \\ \text { M-130 } & \text { Major constituents } \\ \text { N-42 } & \text { Nutrients } \\ \text { P-22 } & \text { Low ionic strength (precipitation) } \\ \text { Hg-18 } & \text { Mercury }\end{array}$

The USGS requested that analytical results be returned by June 17, 1994 for evaluation and preparation of this report. Each participating laboratory is requested to perform those determinations routinely made on the respective SRS for USGS investigations and to indicate the 
analytical method used to determine the concentration of each analyte. When analytical-method information was provided, it has been included in the respective data table. The analytical data are presented in ways that allow participants to evaluate data distribution, scatter, outliers, central tendency, bias, skewness, and method relationships.

Table 1.--Laboratory participants in the analyses of standard reference samples distributed in April 1994

\begin{tabular}{|c|c|c|}
\hline State & City & Participating Laboratory \\
\hline Alabama & Tuscaloosa & Geological Survey of Alabama \\
\hline \multirow{3}{*}{ Arizona } & Phoenix & Arizona Department of Health Services \\
\hline & Phoenix & Nestech Laboratories, Inc. \\
\hline & Yuma & Burns and Roe Services Corporation \\
\hline \multirow[t]{3}{*}{ Arkansas } & Arkadelphia & Ouachita Baptist University \\
\hline & Fayetteville & University of $\mathbf{A r}$ \\
\hline & Little Rock & Arkansas Department of Pollution Control and Ecology \\
\hline \multirow[t]{16}{*}{ California } & Castiac & Castaic Chemical Laboratory, Department of Water Resources \\
\hline & Davis & University of California - Davis \\
\hline & La Verne & Metropolitan Water District of Southern California \\
\hline & Lakeside & Helix Water District \\
\hline & Martinez & Central Contra Costa Sanitary District \\
\hline & Oakland & East Bay Muncipal Utility District \\
\hline & Riverside & University of California - Riverside \\
\hline & Sacramento & Anlab \\
\hline & Sacramento & US Bureau of Reclamation \\
\hline & Sacramento & USGS WRD \\
\hline & San Diego & USGS WRD \\
\hline & Santa Barbara & University of California - Santa Barbara \\
\hline & Santa Fe Springs & West Coast Analytical Service, Inc. \\
\hline & Three Rivers & Southern Sierra Research Center \\
\hline & West Sacramento & California Department of Water Resources \\
\hline & West Sacramento & Enseco - CALLAB \\
\hline \multirow[t]{16}{*}{ Colorado } & Alamosa & US Bureau of Reclamation \\
\hline & Arvada & Enseco - Rocky Mountain Analytical Laboratory \\
\hline & Arvada & USGS National Water Quality Laboratory \\
\hline & Aurora & Core Laboratories, Inc. \\
\hline & Denver & US Bureau of Reclamation \\
\hline & Denver & Denver Water Department \\
\hline & Denver & Metro Wastewater Reclamation \\
\hline & Denver & USGS - Hydrologic Research Unit \\
\hline & Denver & USGS \\
\hline & Denver & USGS WRD \\
\hline & Fort Collins & City of Fort Collins - Water Quality \\
\hline & Fort Collins & USDA US Forest Service \\
\hline & Golden & EG \& G Rocky Flats \\
\hline & Northglenn & Northglenn Water Treatment Plant \\
\hline & Pueblo & Pueblo Board of Water Works \\
\hline & Westminster & City of Westminster \\
\hline \multirow[t]{9}{*}{ Florida } & Brooksville & SW Florida Water Management District \\
\hline & Ft. Lauderdale & Spectrum Laboratories, Inc. \\
\hline & Ocala & USGS WRD \\
\hline & Orlando & Post, Bucklye, Schuh, and Jernigan, Inc. \\
\hline & Ormand Beach & Environmental Laboratory \\
\hline & Palatka & St. John's River Management District \\
\hline & Tallahassee & City of Tallahassee \\
\hline & Tallahassee & Florida Department of Environmental Regulations \\
\hline & Tallahassee & Savannah Laboratories \\
\hline
\end{tabular}


Table 1.--Laboratory participants in the analyses of standard reference samples distributed in April 1994--Continued

\begin{tabular}{|c|c|c|}
\hline State & City & Participating Laboratory \\
\hline \multirow[t]{2}{*}{ Florida } & Tampa & Hillsborough County Environmental Protection Commission \\
\hline & West Palm Beach & South Florida Water Management District \\
\hline \multirow[t]{5}{*}{ Georgia } & Athens & University of Georgia \\
\hline & Atlanta & Georgia Department of Natural Resources \\
\hline & Atlanta & USGS WRD \\
\hline & Decatur & Dekalb County Water Quality Laboratory \\
\hline & Tifton & USDA - Agriculture Research Station \\
\hline \multirow[t]{2}{*}{ Hawaii } & Honolulu & University of Hawaii - SOEST Analytical Services \\
\hline & & Atomic Spectroscopy Laboratory \\
\hline Idaho & Boise & US Bureau of Reclamation \\
\hline \multirow[t]{2}{*}{ Illinois } & Champaign & Hazardous Waste Research Center \\
\hline & Chicago & Illinois Environmental Protection Agency \\
\hline \multirow[t]{2}{*}{ Indiana } & Indianapolis & Indianapolis Department of Public Works \\
\hline & Valparaiso & Coast to Coast Analytical Services, Inc. \\
\hline Iowa & Des Moines & University Hygienic Laboratory, Des Moines Branch \\
\hline \multirow[t]{3}{*}{ Kansas } & Lawrence & Kansas Geological Survey \\
\hline & Topeka & City of Topeka \\
\hline & Topeka & Kansas Department of Health and Environment \\
\hline \multirow[t]{3}{*}{ Kentucky } & Frankfort & Division of Environmental Services \\
\hline & Lexington & Kentucky Geological Survey \\
\hline & Louisville & Metropolitan Sewer District \\
\hline \multirow[t]{2}{*}{ Maine } & Orono & Sawyer Environmental Center, University of Maine \\
\hline & Orono & University of Maine \\
\hline \multirow[t]{2}{*}{ Maryland } & Baltimore & Martel Laboratory Services, Inc. \\
\hline & Baltimore & Maryland Department of Health and Mental Hygiene \\
\hline Massachusetts & Wellesley Hills & Massachusetts Highway Department \\
\hline \multirow[t]{2}{*}{ Michigan } & Ann Arbor & University of Michigan - Department of Geological Science \\
\hline & Detroit & Detroit Water and Sewerage Department \\
\hline \multirow[t]{3}{*}{ Minnesota } & Minneapolis & Braun Intertec Environmental, Inc. \\
\hline & Minneapolis & University of Minnesota, Department of Geology and Geophysics \\
\hline & St. Paul & Metropolitan Waste Control Commission \\
\hline Missouri & Jefferson City & Missouri Department of Health \\
\hline Montana & Helena & Department of Health and Environment \\
\hline \multirow[t]{5}{*}{ Nevada } & Boulder City & US Bureau of Reclamation \\
\hline & Las Vegas & University of Nevada - Las Vegas \\
\hline & Reno & Desert Research Institute \\
\hline & Reno & Nevada State Health Laboratory \\
\hline & Reno & Reno-Sparks Wastewater Treatment \\
\hline \multirow[t]{2}{*}{ New Jersey } & Lydnhurst & Hackesack Meadowlands Center \\
\hline & Trenton & New Jersey Department of Health \\
\hline New Mexico & Albuquerque & City of Albuquerque \\
\hline \multirow[t]{12}{*}{ New York } & Albany & USGS WRD \\
\hline & Brockport & State University of New York - Brockport \\
\hline & Buffalo & Erie County Laboratory \\
\hline & Grahamsville & New York City Department of Environmental Protection \\
\hline & Hempstead & Nassau County Department of Health \\
\hline & Milbrook & Institute of Ecosystem Studies \\
\hline & Oakdale & Suffolk County Water Authority \\
\hline & Port Washington & New York Test Environmental, Inc. \\
\hline & Rochester & Monroe County \\
\hline & Shokan & New York City Department of Environmental Protection \\
\hline & Syracuse & Onondaga County Department of Drainage and Sanitation \\
\hline & Syracuse & State University of New York - Syracuse \\
\hline
\end{tabular}


Table 1.--Laboratory participants in the analyses of standard reference samples distributed in April 1994-Continued

\begin{tabular}{|c|c|c|}
\hline State & City & Participating Laboratory \\
\hline \multirow[t]{2}{*}{ New York } & Valhalla & Department of Environmental Protection \\
\hline & Wantaugh & Cedar Creek Projects Laboratory \\
\hline \multirow[t]{4}{*}{ North Carolina } & Charlotte & Mecklenburg County - Department of Environmental Protection \\
\hline & Durham & Department of Water Resources \\
\hline & Durham & Duke University \\
\hline & Greensboro & City of Greensboro \\
\hline \multirow{3}{*}{ North Dakota } & Bismarck & North Dakota State Health Department \\
\hline & Bismarck & North Dakota State Water Commission \\
\hline & Bismarck & US Bureau of Reclamation \\
\hline \multirow[t]{3}{*}{ Ohio } & Columbus & City of Columbus \\
\hline & Medina & Medina County Sanitary Engineering \\
\hline & Tiffin & Heidelberg College \\
\hline Oklahoma & Norman & Oklahoma Geological Survey \\
\hline \multirow[t]{2}{*}{ Oregon } & Corvallis & US Department of Agriculture \\
\hline & Tigard & Unified Sewerage Agency \\
\hline \multirow[t]{2}{*}{ Pennsylvania } & Harrisburg & Pennsylvania Department of Environmental Resources \\
\hline & Somerset & Geochemical Testing \\
\hline Puerto Rico & San Juan & Department of Natural Resources \\
\hline \multirow[t]{3}{*}{ South Dakota } & Brookings & Northern Great Plains Laboratory \\
\hline & Brookings & SDSU - Water Quality Laboratory \\
\hline & Vermillion & South Dakota Geologiical Survey \\
\hline Tennessee & Chattanooga & Tennessee Valley Authority, Environmental Chemistry \\
\hline \multirow[t]{2}{*}{ Texas } & San Antonio & Pollution Control Services \\
\hline & Tyler & Analytical Testing Laboratories \\
\hline Vermont & Waterbury & Vermont Agency of Natural Resources \\
\hline \multirow[t]{5}{*}{ Virginia } & Culpepper & ESS Laboratories \\
\hline & Manassas & Occoquan Watershed Monitoring Laboratory \\
\hline & Reston & USGS WRD \\
\hline & Richmond & Consolidated Laboratory Services \\
\hline & Virginia Beach & Hampton Road Sanitation District \\
\hline \multirow[t]{2}{*}{ Washington } & Richland & Battelle - Pacific Northwest \\
\hline & Seattle & Brooks-Rand, Ltd. \\
\hline West Virginia & Morgantown & University of West Virginia \\
\hline \multirow[t]{3}{*}{ Wisconsin } & Green Bay & Green Bay Metro Sewerage District \\
\hline & Madison & State Laboratory of Hygiene \\
\hline & Milwaukee & Milwaukee Metro Sewerage District \\
\hline Wyoming & Laramie & Wyoming Department of Agriculture \\
\hline
\end{tabular}

\section{Preparation of Standard Reference Samples}

All of the SRS used in this evaluation were prepared by personnel of the USGS in Golden, Colo. and were analyzed for analyte concentrations and physical property values prior to mailing.

Trace constituent sample T-129 was prepared using water collected from the Clear Creek near Idaho Springs, Colorado. The water was pumped through $2-$ and $0.1-\mu \mathrm{m}$ filters, in series, into a $1300-\mathrm{L}$ polypropylene drum. The water was continuously circulated and passed through a $0.1-\mu \mathrm{m}$ filter and ultraviolet sterilizer for 24 hours. Following this circulation, the water was acidified to $\mathrm{pH} 1.5$ with nitric acid and chlorinated to 5-ppm free chlorine with sodium hypochlorite. The sample was circulated an additional 24 hours prior to bottling. During bottling the sample was pumped through an ultraviolet sterilizer and a $0.1-\mu \mathrm{m}$ filter. The $500-\mathrm{mL}$ polypropylene bottles 
used were acid leached, deionized-water rinsed, and autoclave sterilized. Bottles not mailed for this SRS evaluation are stored until requested for use.

Major constituent sample M-130 was prepared using water collected from the Clear Creek near Idaho Springs, Colorado. The water was pumped through $2-$ and $0.1-\mu \mathrm{m}$ filters, in series, into a 1300-L polypropylene drum. The water was chlorinated to 5-ppm free chlorine with sodium hypochlorite, continuously circulated, and passed through a $0.1-\mu \mathrm{m}$ filter and ultraviolet sterilizer for 24 hours prior to bottling. During bottling the sample was pumped through an ultraviolet sterilizer and a $0.1-\mu \mathrm{m}$ filter. The $500-\mathrm{mL}$ polypropylene bottles used were acid leached, deionized-water rinsed, and autoclave sterilized. Bottles not mailed for this SRS evaluation are stored until requested for use.

Nutrient sample N-42 was prepared using water collected from the Fall River near Idaho Springs, Colorado. These samples were prepared the week prior to the mailing for this SRS evaluation. The water was pumped through $2-$ and $0.1-\mu \mathrm{m}$ filters, in series, into a $600-\mathrm{L}$ polypropylene drum and continuously circulated and passed through a $0.1-\mu \mathrm{m}$ filter for 48 hours. The desired nutrient concentrations were obtained by adding reagent-grade chemicals. The sample was circulated an additional 24 hours. A number of nonpreserved samples were bottled from this solution and refrigerated at $4{ }^{\circ} \mathrm{C}$. The remaining sample was preserved with mercuric chloride, to a concentration of $50 \mathrm{mg} / \mathrm{L}$, and with sodium chloride, to a concentration of $450 \mathrm{mg} / \mathrm{L}$. The preserved sample was continuously circulated for 24 hours prior to being bottled. The $250-\mathrm{mL}$ polyethylene bottles used were new, amber, acid leached, and deionized-water rinsed. Bottles not mailed for this SRS evaluation are refrigerated at $4{ }^{\circ} \mathrm{C}$ until requested for use.

Sample P-22 was prepared in a 400-L polypropylene drum using snow collected at Mt. Evans near Summit Lake in Colorado. The collected snow was allowed to melt; then it was pumped into the drum through 2- and 0.1- $\mu \mathrm{m}$ filters in series. Desired phosphate and fluoride concentrations were obtained by adding reagent-grade chemicals. Prior to bottling, the sample was continuously mixed for 48 hours while being circulated through a $0.1-\mu \mathrm{m}$ filter and an ultraviolet sterlizer. During bottling the sample was pumped through an ultraviolet sterilizer and a $0.1-\mu \mathrm{m}$ filter. The $500-\mathrm{mL}$ polypropylene bottles used were acid leached, deionized-water rinsed, and autoclave sterilized. Bottles not mailed for this SRS evaluation are stored until requested for use.

Sample Hg-18 was prepared using water collected from the Fall River near Idaho Springs, Colorado. The sample was prepared in a $190-\mathrm{L}$ polypropylene drum. The river water was pumped into this drum through $2-$ and $0.1-\mu \mathrm{m}$ filters in series. The water was continuously circulated and passed through a $0.1-\mu \mathrm{m}$ filter and ultraviolet sterilizer for 72 hours. Nitric acid (5-percent, v/v) and dichromate ion ( 0.05 -percent, $w / w)$ were added to stabilize the sample. The desired mercury concentration was obtained by adding a mercury standard solution. Following an additional 24 hours of circulation, the sample was bottled. The $125-\mathrm{mL}$ glass bottles and tetrafluoroethylene fluorocarbon resin caps used were new, acid leached, and deionized-water rinsed. Bottles not mailed for this SRS evaluation are stored until requested for use. 


\section{LABORATORY ANALYSES}

The participating laboratories were asked to determine analytes which are summarized in table 2 . The number of analytes varied from 26 in T-129 (trace constituents) to 1 in $\mathrm{Hg}-18$ (mercury).

Table 2.--Analytes determined in standard reference samples distributed in April 1994

\begin{tabular}{|c|c|c|c|c|c|c|c|}
\hline \multicolumn{2}{|c|}{ Analyte or property } & Units & $T-129$ & $M-130$ & $\mathrm{~N}-42$ & $P-21$ & $\mathrm{Hg}-18$ \\
\hline Acidity & Acidity as $\mathrm{CaCO}_{3}$ & $\mathrm{mg} / \mathrm{L}$ & & & & $\mathrm{X}$ & \\
\hline Alk & Alkalinity as $\mathrm{CaCO}_{3}$ & $\mathrm{mg} / \mathrm{L}$ & & $\mathrm{x}$ & & & \\
\hline Ag & Silver & $\mu \mathrm{g} / \mathrm{L}$ & $\mathrm{X}$ & & & & \\
\hline Al & Aluminum & $\mu \mathrm{g} / \mathrm{L}$ & $\mathrm{x}$ & & & & \\
\hline As & Arsenic & Hg/L & $x$ & & & & \\
\hline B & Boron & $\mu \mathrm{g} / \mathrm{L}$ & $\mathrm{x}$ & $\mathrm{x}$ & & & \\
\hline $\mathbf{B a}$ & Barium & $\mu \mathrm{g} / \mathrm{L}$ & $\mathrm{x}$ & & & & \\
\hline $\mathrm{Be}$ & Beryllium & $\mu \mathrm{g} / \mathrm{L}$ & $\mathrm{x}$ & & & & \\
\hline $\mathrm{Ca}$ & Calcium & $\mathrm{mg} / \mathrm{L}$ & $\mathrm{x}$ & $\mathrm{x}$ & & $\mathrm{x}$ & \\
\hline $\mathrm{Cd}$ & Cadmium & $\mu \mathrm{g} / \mathrm{L}$ & $\mathrm{x}$ & & & & \\
\hline $\mathrm{Cl}$ & Chloride & $\mathrm{mg} / \mathrm{L}$ & & $\mathrm{x}$ & & $\mathrm{x}$ & \\
\hline Co & Cobalt & $\mu \mathrm{g} / \mathrm{L}$ & $\mathrm{x}$ & & & & \\
\hline $\mathrm{Cr}$ & Chromium, total & $\mu \mathrm{g} / \mathrm{L}$ & $\mathrm{x}$ & & & & \\
\hline $\mathrm{Cu}$ & Copper & $\mu \mathrm{g} / \mathrm{L}$ & $\mathrm{x}$ & & & & \\
\hline DSRD & Dissolved solids & $\mathrm{mg} / \mathrm{L}$ & & $x$ & & & \\
\hline $\mathbf{F}$ & Fluoride & $\mathrm{mg} / \mathrm{L}$ & & $x$ & & $\mathrm{x}$ & \\
\hline $\mathrm{Fe}$ & Iron & $\mu \mathrm{g} / \mathrm{L}$ & $\mathrm{x}$ & & & & \\
\hline Hg & Mercury & $\mu \mathrm{g} / \mathrm{L}$ & & & & & $\mathrm{X}$ \\
\hline $\mathbf{K}$ & Potassium & $\mathrm{mg} / \mathrm{L}$ & $\mathrm{x}$ & $\mathbf{X}$ & & $\mathrm{x}$ & \\
\hline $\mathrm{Li}$ & Lithium & $\mu \mathrm{g} / \mathrm{L}$ & $x$ & & & & \\
\hline Mg & Magnesium & $\mathrm{mg} / \mathrm{L}$ & $\mathbf{X}$ & $\mathrm{x}$ & & $\mathrm{x}$ & \\
\hline Mn & Manganese & $\mu \mathrm{g} / \mathrm{L}$ & $\mathrm{x}$ & & & & \\
\hline Mo & Molybdenum & $\mu \mathrm{g} / \mathrm{L}$ & $\mathrm{x}$ & & & & \\
\hline $\mathrm{Na}$ & Sodium & $\mathrm{mg} / \mathrm{L}$ & $\mathbf{X}$ & $\mathbf{X}$ & & $\mathrm{x}$ & \\
\hline $\mathrm{NH}_{3}$ as $\mathrm{N}$ & Ammonia & $\mathrm{mel}$ & & & $x$ & & \\
\hline $\mathrm{NH}_{3}+\mathrm{Org} \mathrm{N}$ as $\mathrm{N}$ & Ammonia + Organic $\mathbf{N}$ & $\mathrm{mg} / \mathrm{L}$ & & & $\mathrm{x}$ & & \\
\hline $\mathrm{Ni}$ & Nickel & $\mu \mathrm{g} / \mathrm{L}$ & $\mathrm{x}$ & & & & \\
\hline $\mathrm{NO}_{3}+\mathrm{NO}_{2}$ as $\mathrm{N}$ & Nitrate + Nitrite & $\mathrm{mg} / \mathrm{L}$ & & & $\mathrm{x}$ & $\mathbf{x}$ & \\
\hline $\mathrm{Pb}$ & Lead & $\mu \mathrm{g} / \mathrm{L}$ & $\mathrm{x}$ & & & & \\
\hline $\mathrm{pH}$ & & unit & & $x$ & & $x$ & \\
\hline $\mathrm{PO}_{4}$ as $\mathrm{P}$ & Orthophosphate & $\mathrm{mg} / \mathrm{L}$ & & & $x$ & $\mathrm{x}$ & \\
\hline total $P$ as $P$ & Phosphorus & $\mathrm{mg} / \mathrm{L}$ & & $\mathrm{X}$ & $\mathrm{x}$ & & \\
\hline $\mathrm{Sb}$ & Antimony & $\mu \mathrm{g} / \mathrm{L}$ & $\mathrm{x}$ & & & & \\
\hline $\mathrm{Se}$ & Selenium & $\mu \mathrm{g} / \mathrm{L}$ & $\mathrm{x}$ & & & & \\
\hline $\mathrm{SiO}_{2}$ & Silica & $\mathrm{mg} / \mathrm{L}$ & $x$ & $x$ & & & \\
\hline $\mathrm{SO}_{4}$ & Sulfate & $\mathrm{mg} / \mathrm{L}$ & & $x$ & & $\mathrm{x}$ & \\
\hline Sp Cond & Specific conductance & $\mu \mathrm{S} / \mathrm{cm}$ & & $\mathbf{x}$ & & $\mathrm{x}$ & \\
\hline $\mathrm{Sr}$ & Strontium & $\mu \mathrm{g} / \mathrm{L}$ & $\mathrm{x}$ & $\mathbf{X}$ & & & \\
\hline $\mathrm{v}$ & Vanadium & $\mu \mathrm{g} / \mathrm{L}$ & $\mathrm{x}$ & $\mathrm{x}$ & & & \\
\hline $\mathrm{Zn}$ & Zinc & ng/L & $x$ & & & & \\
\hline
\end{tabular}


Laboratories were requested to identify the method used for each analyte according to table 3 analytical method codes.

Table 3.--Analytical-method codes

\begin{tabular}{ll} 
Code & Method \\
\hline 0 & Other \\
1 & Atomic absorption: direct, air \\
2 & Atomic absorption: direct, nitrous oxide \\
3 & Atomic absorption: graphite furnace \\
4 & Inductively coupled argon plasma \\
5 & Direct current plasma \\
6 & Inductively coupled argon plasma/Mass spectrometry \\
7 & Ion chromatography \\
8 & Atomic absorption: cold vapor \\
10 & Atomic absorption: extraction [specify chelating agents ] \\
11 & Atomic absorption: hydride [specify reducing agent ] \\
12 & Flame emission \\
20 & Titration: colorimetric [specify color reagent ] \\
22 & Colorimetric: [specify reducing or oxidizing agent/color reagent ] \\
40 & Ion selective electrode \\
41 & Electrometric [pH and Specific Conductance] \\
50 & Gravimetric: [specify filtration, evaporation, and so forth] \\
51 & Turbidimetric
\end{tabular}

Participating laboratories were also asked to use the references listed below to further define the methods.

1. American Public Health Association and others, 1989, Standard methods for the examination of water and wastewater 17th ed: Washington, D.C., American Public Health Association, 1527p.

2. American Society for Testing and Materials, Annual book of ASTM standards:

Philadelphia, v. 11.01, and v. 11.02.

3. Kopp, J.F., and McKee, G.F., 1979, Methods for chemical analysis of water and wastes: Cincinnati, U.S. Environmental Protection Agency, EPA 600/4-79-020, rev. 1983, $460 \mathrm{p}$.

4. Fishman, M.J., and Friedman, L.C., eds., 1989. Methods for determination of inorganic substances in water and fluvial sediments ( $3 \mathrm{~d}$ ed.): U.S. Geological Survey Techniques of Water-Resources Investigations, Book 5, Chapter A1, 545 p.

5. Miscellaneous manufacturer's instrument manuals or references. 
To facilitate interlaboratory performance comparisons, laboratory performance ratings, based on the analyses reported for each SRS, are included in tables 4 through 12 in this report. Averages of the analyte ratings and the number of analyte values reported for each SRS are given for each participating laboratory. Laboratory performance for each analyte is rated on a scale 4 to 0 , based on the absolute Z-value, as listed below:

\begin{tabular}{ll}
\hline Rating & Absolute Z-value \\
4 (Excellent) & 0.00 to 0.50 \\
3 (Good) & 0.51 to 1.00 \\
2 (Satisfactory) & 1.01 to 1.50 \\
1 (Questionable) & 1.51 to 2.00 \\
0 (Poor) & Greater than 2.00 \\
\hline
\end{tabular}

Overall laboratory performance ratings greater than 2.4 are considered satisfactory. Overall laboratory performance ratings between 2.0 and 2.39 are considered marginal; those less than 2.0 are considered poor.

\section{STATISTICAL PRESENTATION OF DATA}

Data in this report have been evaluated using nonparametric statistics as described by Hoaglin and others (1983). This statistical approach is a resistant statistic because the median is not influenced by outliers as is the mean in traditional statistics.

Analytical data for each analyte are presented in tabular and graphical forms in tables 10 through 14. Tabulated data for each analyte include the laboratory code number, reported values, analytical method, most probable value (MPV), number of reported values $(N)$, less than values $(<)$, data range, $Z$-value, and the F-pseudosigma. (The $Z$-value is equivalent to the $Z$-score of traditional statistics, being the number of deviations the reported value is from the MPV. The F-pseudosigma is equivalent to the standard deviation $(\sigma)$ of traditional statistics when the data has a Gaussian distribution.) If an analyte has a sufficient number of determinations by a given method, usually 7 , the $\sigma$ for that analytical method is reported in the block of data listed for each analyte.

The median value is considered the MPV. Reported values of "less than" are not used in this report to establish the median or the data range. The median (midpoint) divides the ordered data into halves and is designated the MPV. The hinges include the middle 50-percent of the data and are the mid-values of the upper and lower halves of the data. (The hinges are similar to quartiles, but are not mathematically equivalent.) The range of data between the upper hinge $(\mathrm{Hu})$ and the lower hinge ( $\mathrm{HI})$, the hinge spread (H-spr), is used to calculate the F-pseudosigma, the laboratory performance rating, the upper warning level (UWL) and lower warning level (LWL), the upper control level (UCL) and the lower control level (LCL). The F-pseudosigma is calculated by comparison of the $\mathrm{H}$-spr value to the Gaussian distribution relation; 67.45 percent of the data "hinges" between plus and minus $1 \sigma$, resulting in a $\mathrm{H}$-spr of $2 \times 0.6745=1.349 \sigma$, This relation allows the calculation of the F-pseudosigma $=(\mathrm{H}$-spr $) / 1.349$. Laboratories reporting "less than" values are not performance rated unless their reported "less than" values are more than two Zvalues from the MPV. 
The graphical plot of the reported data is shown in figure 1. The upper and lower boundaries of the graphical plots generally are +3 and -3 F-pseudosigma deviations from the median. (Computer-program scaling constraints do not permit these boundaries to always be graphed at exactly these values.) The graphical plot is a box plot/control chart with reported values grouped by analytical method in ascending order of value. Lines designate the MPV, Hu, HI, and the (UWL) and (LWL) at +2 and -2 F-pseudosigma, respectively. "Less than" values are not plotted.

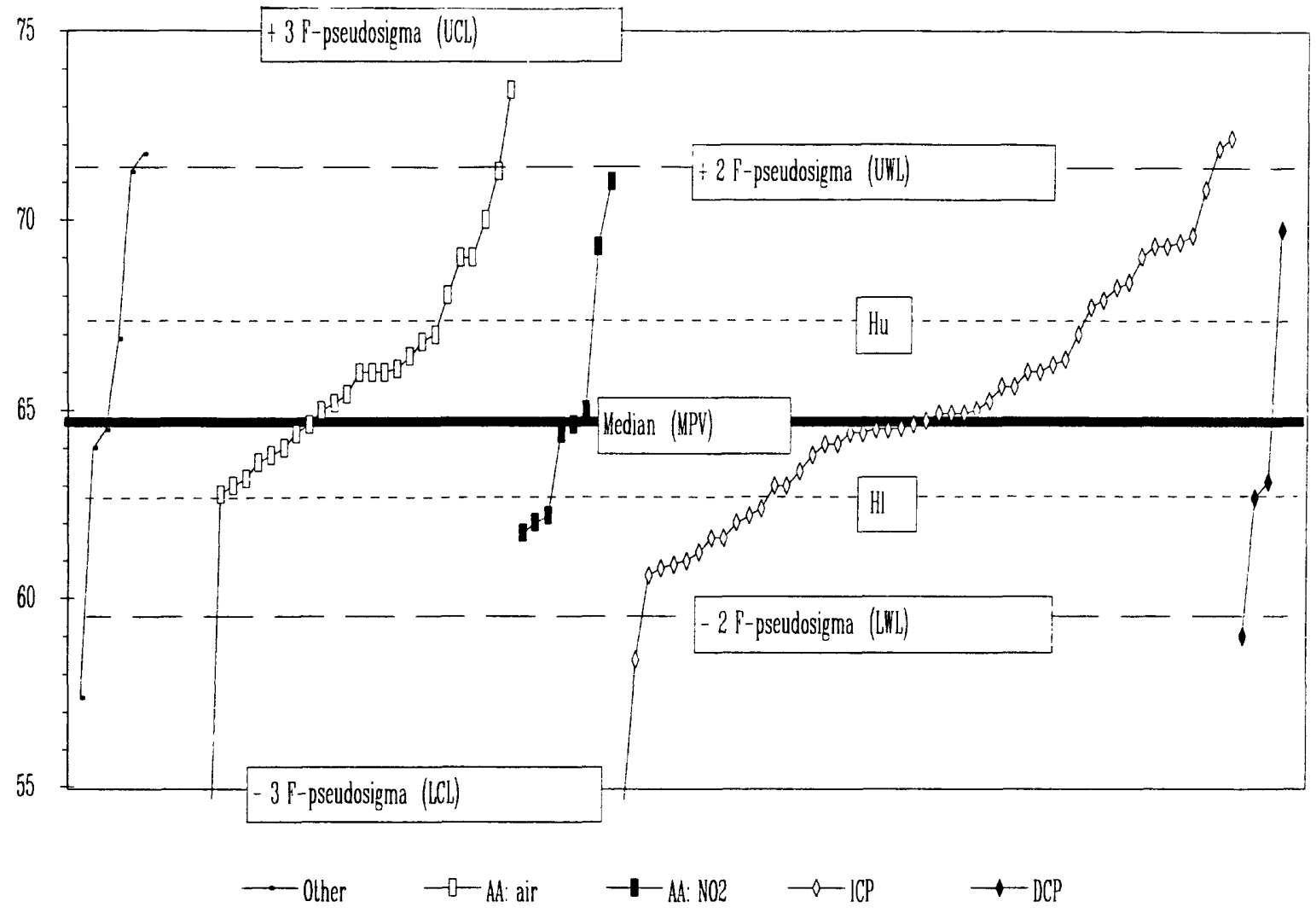

NOTE: vertical scale is the concentration value of the individual analyte in appropriate units (see table 2.) Methods shown are defined in Tables 3 and 10 through 14.

Figure 1.-Statistical parameters shown on reported-data graphs

\section{DISCUSSION}

In this report, less than values have not been used to establish MPV's for T-129 analytes. This sample has low concentrations for many of the trace analytes. Many of the laboratories do not routinely report concentrations at these low levels, therefore, the many less than values have not been incorporated into the statistics.

Users need to review the tabulated and graphical plots for individual analytes because these tables and plots give indications of the method and instrumentation precision, and help provide additional evidence as to the desirability of upgrading methods or equipment or both.

\section{REFERENCE}

Hoaglin, D.C., Mosteller, F., and Tukey, J.W., eds., 1983, Understanding Robust and Exploratory Data Analysis: John Wiley and Sons, Inc., 447p. 
Table 4. - Overall laboratory performance ratings for standard reference water samples distributed in April 1994

[Lab, laboratory number; OWR, overall weighted rating for all sample types; OLR, overall laboratory rating for raported values of a sample type;

V/63, number of raported values of 63 total possibla values from all sample types; V/26, V/15, V/10,V/11,V/1 are number of reportad

values possible for T-129, M-130, N-42, P-22, and $\mathrm{Hg}-18$ respectivaly]

\begin{tabular}{|c|c|c|c|c|c|c|c|c|c|c|c|c|}
\hline \multicolumn{3}{|c|}{ Standard reference sample = } & \multicolumn{2}{|c|}{$1-129$} & \multicolumn{2}{|c|}{$M-130$} & \multicolumn{2}{|c|}{$\mathrm{N}-42$} & \multicolumn{2}{|c|}{ P-22 } & \multicolumn{2}{|c|}{$\mathrm{Hg}-18$} \\
\hline Lab & OWR & V/63 & OLR & V/26 & OLR & V/15 & OLR & $v / 10$ & OLR & V/11 & OLR & $\mathrm{v} / 1$ \\
\hline 1 & 3.5 & 53 & 37 & 22 & 3.4 & 15 & 3.6 & 5 & 3.0 & 10 & 3 & 1 \\
\hline 2 & 1.9 & 7 & & & & & & & 1.9 & 7 & & \\
\hline 3 & 2.5 & 44 & 26 & 16 & 2.7 & 15 & 30 & 5 & 1.6 & 7 & 3 & 1 \\
\hline 4 & 1.8 & 17 & 12 & 9 & 2.5 & 8 & & & & & & \\
\hline 5 & 2.7 & 38 & 3,4 & 13 & 2.6 & 13 & 22 & 6 & 1.7 & 7 & & \\
\hline 6 & 2.3 & 20 & 24 & 9 & 2.4 & 7 & 16 & 4 & & & & \\
\hline 7 & 2.6 & 34 & 28 & 14 & 2.5 & 11 & 2.5 & 4 & 3.0 & 4 & 2 & 1 \\
\hline 9 & 2.7 & 22 & 26 & 8 & 2.7 & 9 & 32 & 5 & & & & \\
\hline 10 & 3.8 & 20 & 3.3 & 3 & 3.9 & 12 & 3.6 & b & & & & \\
\hline 11 & 2.3 & 52 & 24 & 17 & 2.5 & 15 & 12 & 10 & 2.9 & 9 & 4 & 1 \\
\hline 12 & 3.1 & 24 & 33 & 8 & 3.0 & 11 & 3.3 & 4 & & & 3 & 1 \\
\hline 13 & 2.7 & 27 & 21 & 9 & 3.0 & 13 & 40 & 4 & & & o & 1 \\
\hline 15 & 2.5 & 46 & 2,7 & 13 & 1.8 & 14 & 3.5 & 8 & 2.5 & 10 & 4 & 1 \\
\hline 19 & 2.5 & 21 & 27 & 7 & 2.3 & 10 & 28 & 4 & & & & \\
\hline 21 & 3.0 & 4 & 3.0 & 1 & & & 3.0 & 3 & & & & \\
\hline 22 & 4.0 & 1 & & & & & 4.0 & 1 & & & & \\
\hline 23 & 2.9 & 43 & 2.6 & 14 & 2.7 & 13 & 4.0 & 5 & 3.1 & 11 & & \\
\hline 24 & 3.2 & 37 & 32 & 24 & 3.0 & 12 & & & & & 4 & 1 \\
\hline 25 & 2.3 & 37 & 19 & 10 & 2.2 & 13 & 2.5 & 4 & 2.8 & 10 & & \\
\hline 26 & 1.8 & 22 & 20 & $\begin{array}{l}3 \\
\end{array}$ & 1.7 & 11 & 30 & 3 & 1.0 & 5 & & \\
\hline 30 & 3.2 & 14 & 3.6 & 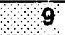 & 2.6 & 5 & & & & & & \\
\hline 32 & 2.8 & 34 & 24 & 16 & 2.9 & 14 & 40 & 3 & & & 4 & 1 \\
\hline 33 & 3.0 & 29 & 26 & 10 & 3.3 & 10 & 10 & 1 & 3.4 & 8 & & \\
\hline 34 & 3.7 & 3 & 3.5 & 2 & & & & & & & 4 & 1 \\
\hline 36 & 2.3 & 54 & 2.3 & 21 & 2.7 & 13 & 16 & 10 & 2.2 & 9 & 3 & 1 \\
\hline 38 & 3.5 & 23 & & & 3.3 & 10 & 32 & 5 & 4.0 & 8 & & \\
\hline 39 & 3.5 & 22 & 3.8 & 13 & 3.8 & 5 & & & 3.3 & 3 & 0 & 1 \\
\hline 40 & 3.3 & 13 & & $\mathbf{l}^{?}$ & 3.3 & 13 & & & & & & \\
\hline 43 & 3.3 & 18 & 3.8 & 6 & 3.3 & 11 & 1.0 & 1 & & & & \\
\hline 44 & 2.8 & 6 & - & & & & & & 2.8 & 6 & & \\
\hline 45 & 3.0 & 28 & 2.8 & 12 & 3.3 & 11 & 2.8 & 4 & & & 2 & 1 \\
\hline 46 & 3.6 & 38 & 37 & 11 & 3.7 & 13 & 36 & 5 & 3.4 & 8 & 4 & 1 \\
\hline 48 & 2.2 & 36 & 27 & 12 & 1.7 & 10 & 2.6 & 5 & 1.8 & 8 & 4 & 1 \\
\hline 50 & 2.9 & 17 & 20 & 4 & 3.3 & 12 & & & & & 1 & 1 \\
\hline 51 & 2.3 & 32 & 2,3 & 16 & 2.2 & 11 & 28 & 6 & & & 2 & 1 \\
\hline 52 & 2.8 & 47 & 26 & 16 & 2.4 & 13 & 3.8 & 10 & 3.0 & 7 & 1 & 1 \\
\hline 53 & 2.0 & 2 & & & & & 2.0 & 2 & & & & \\
\hline 54 & 3.3 & 15 & 28 & 4 & 3.7 & 10 & $1: 0$ & 1 & & & & \\
\hline 55 & 3.0 & 25 & 2.6 & 9 & 3.0 & 12 & 3.7 & $\mathbf{3}$ & & & 4 & 1 \\
\hline 56 & 1.7 & 13 & 26 & & 2.2 & 9 & 0.5 & 4 & & & & \\
\hline 58 & 1.5 & 54 & 1.8 & 24 & 0.8 & 13 & 18 & 5 & 1.5 & 11 & 3 & 1 \\
\hline 59 & 3.3 & 16 & 31 & 10 & & & 34 & 5 & & & 4 & 1 \\
\hline 61 & 2.6 & 26 & 2.7 & 10 & & & 2,4 & $\mathbf{b}$ & 2.6 & 10 & 3 & 1 \\
\hline 62 & 3.5 & 2 & & & & & & & 3.5 & 2 & & \\
\hline 63 & 2.5 & 42 & 22 & 10 & 2.4 & 12 & 2.6 & 10 & 2.6 & 9 & 3 & 1 \\
\hline 64 & 3.2 & 25 & 36 & 4 & 3.0 & 8 & 20 & 4 & 3.6 & 9 & & \\
\hline 68 & 2.8 & 33 & 2.8 & 16 & 3.1 & 11 & 2.6 & b & & & 1 & 1 \\
\hline 69 & 2.6 & 19 & 23 & 6 & 2.6 & 11 & 30 & 1 & & & 4 & 1 \\
\hline 70 & 3.1 & 29 & 3.4 & $\theta$ & 3.2 & 14 & 22 & E & & & 3 & 1 \\
\hline 72 & 2.0 & 22 & 07 & 7 & 2.8 & 10 & 22 & 5 & & & & म \\
\hline 73 & 3.0 & 5 & 3.0 & 6 & & & & & & & & \\
\hline 75 & 3.3 & 30 & 31 & 11 & 3.6 & 10 & 3.1 & $B$ & & & 4 & 1 \\
\hline 76 & 3.0 & 13 & & & 3.1 & 10 & 2.5 & 2 & & & 3 & 1 \\
\hline 78 & 2.7 & 55 & 32 & 21 & 2.1 & 13 & 23 & 10 & 2.8 & 10 & 4 & 1 \\
\hline 79 & 2.5 & 13 & 17 & 6 & 3.5 & 4 & 2.5 & 2 & & & 3 & 1 \\
\hline 80 & 1.9 & 13 & & & 2.3 & 10 & 0.7 & 3 & & & & \\
\hline 81 & 3.7 & 6 & & & & & 3.8 & 5 & & & 3 & 1 \\
\hline 84 & 3.2 & 14 & 33 & 4 & 3.3 & 7 & 3.0 & 3 & & & & \\
\hline 85 & 3.2 & 30 & 33 & 11 & 3.4 & 14 & 2.4 & 5 & & & & \\
\hline 86 & 3.0 & 22 & 36 & 11 & 2.5 & 10 & & 0 & & & 4 & 1 \\
\hline 87 & 2.3 & 33 & 10 & 15 & 2.2 & 12 & 38 & 5 & & & 3 & 1 \\
\hline 88 & 1.3 & 6 & & & & & 13 & 6 & & & & \\
\hline 90 & 2.3 & 24 & 14 & 12 & 2.8 & 6 & 3.8 & 5 & & & 3 & 1 \\
\hline 91 & 2.8 & 4 & 40 & 1 & & & 23 & 3 & & & & \\
\hline 92 & 2.1 & 35 & 15 & 13 & 3.2 & 12 & 17 & 7 & 3.0 & 2 & 0 & 1 \\
\hline 93 & 2.3 & 20 & & & 2.1 & 9 & 2.0 & 2 & 2.6 & 9 & & \\
\hline 94 & 3.1 & 36 & 3.1 & 11 & 3.4 & 13 & 2.5 & 4 & 3.0 & 8 & & \\
\hline 96 & 2.8 & 15 & 30 & 2 & 2.7 & 7 & 26 & 5 & & & 4 & 1 \\
\hline 97 & 2.7 & 39 & 25 & 14 & 2.6 & 14 & 31 & 10 & & & 4 & 1 \\
\hline 100 & 2.6 & 42 & 24 & 17 & 3.1 & 8 & 28 & 10 & 2.3 & 6 & 0 & 1 \\
\hline
\end{tabular}


Table 4. - Overall laboratory performance ratings for standard reference water samples distributed in April 1994 --Continued

\begin{tabular}{|c|c|c|c|c|c|c|c|c|c|c|c|c|}
\hline \multicolumn{3}{|c|}{ Standard reference sample = } & \multicolumn{2}{|c|}{ T 129} & \multicolumn{2}{|c|}{$M-130$} & \multicolumn{2}{|c|}{$\mathrm{N}+2$} & \multicolumn{2}{|c|}{ P-22 } & \multicolumn{2}{|c|}{ Hglo } \\
\hline Lab & OWR & V/63 & OLR & $v_{126}$ & OLR & $V / 15$ & OLR & v/10 & OLR & $V / 11$ & OLR & V/1 \\
\hline 101 & 2.3 & 30 & 2.2 & 15 & 2.4 & 8 & & & 2.6 & 7 & & \\
\hline 102 & 2.3 & 33 & 2.4 & 14 & 2.9 & 9 & 1,8 & 5 & 1.6 & 5 & & \\
\hline 103 & 3.4 & 21 & 3.4 & 14 & 3.6 & 7 & & & & & & \\
\hline 105 & 3.2 & 41 & 3.5 & 21 & 2.9 & 14 & 3.4 & 5 & & & 0 & 1 \\
\hline 107 & 2.9 & 36 & 2.5 & 12 & 2.8 & 11 & 2.3 & 4 & 3.9 & 9 & & \\
\hline 108 & 3.1 & 12 & 3.8 & 6 & 4.0 & 1 & 15 & 4 & & & 4 & G \\
\hline 109 & 3.2 & 27 & 3.3 & 13 & 3.0 & 13 & & & & & 4 & 1 \\
\hline 110 & 3.7 & 9 & & & & & & & 3.7 & 9 & & \\
\hline 111 & 2.2 & 27 & 1.8 & 6 & 1.7 & 10 & 2.3 & 3 & 3.0 & 8 & & \\
\hline 112 & 3.4 & 7 & & & & & & & 3.4 & 7 & & \\
\hline 113 & 2.6 & 39 & 23 & 14 & 2.9 & 14 & 3.3 & 4 & 2.8 & 6 & 0 & 7 \\
\hline 114 & 2.2 & 29 & 1,5 & 12 & 2.7 & 11 & 3.2 & 5 & & & 0 & 1 \\
\hline 116 & 3.2 & 17 & 31 & 9 & 3.4 & 8 & & & & & & \\
\hline 118 & 2.5 & 16 & 10 & 1 & 1.5 & 4 & 33 & 10 & & & 0 & 1 \\
\hline 119 & 3.2 & 40 & 3.3 & 15 & 3.3 & 14 & 2.9 & 10 & & & 4 & 1 \\
\hline 121 & 3.5 & 24 & 34 & 17 & 3.6 & 7 & & & & & & \\
\hline 122 & 3.0 & 22 & 0.0 & 1 & 2.8 & 11 & 3.4 & 10 & & & & \\
\hline 126 & 3.3 & 3 & 40 & 2 & & & 20 & 1 & & & & \\
\hline 127 & 3.2 & 35 & 2.9 & 15 & 3.3 & 14 & 3.8 & 5 & & & 3 & 1 \\
\hline 128 & 2.2 & 29 & 2,5 & 11 & 2.3 & 12 & 2.0 & 5 & & & 0 & 1 \\
\hline 129 & 2.8 & 22 & 0.0 & 2 & 2.6 & 10 & 3.6 & 10 & & & & \\
\hline 131 & 2.3 & 4 & & & 2.3 & 4 & & & & & & \\
\hline 133 & 2.6 & 14 & 3.0 & 4 & 2.3 & 4 & 2.6 & 5 & & & 2 & 1 \\
\hline 134 & 3.5 & 47 & 3,8 & 15 & 3.6 & 13 & 3.6 & 10 & 2.9 & 8 & 4 & 1 \\
\hline 136 & 2.1 & 36 & 19 & 14 & 2.4 & 10 & 3.0 & 3 & 1.8 & 9 & & \\
\hline 138 & 3.2 & 24 & & & 2.9 & 11 & 3.6 & 5 & 3.1 & 7 & 4 & 1 \\
\hline 140 & 2.9 & 39 & 3.2 & 13 & 3.1 & 13 & 20 & 5 & 2.8 & 8 & & \\
\hline 141 & 2.6 & 38 & 25 & 11 & 2.6 & 13 & 3,6 & 5 & 2.1 & 8 & 4 & 1 \\
\hline 142 & 3.0 & 43 & 3.3 & 25 & 3.1 & 12 & 2.0 & 5 & & & 2 & 1 \\
\hline 143 & 3.3 & 14 & & & 3.2 & 5 & 3.6 & 5 & 3.0 & 4 & & \\
\hline 145 & 3.1 & 40 & 3.5 & 10 & 2.8 & 12 & 30 & 10 & 3.3 & 7 & 2 & 1 \\
\hline 146 & 1.6 & 23 & 19 & 10 & 1.2 & 10 & 1,0 & 2 & & & 4 & 1 \\
\hline 149 & 2.8 & 11 & 2.8 & 6 & 2.5 & 4 & & & & & 4 & 1 \\
\hline 151 & 3.3 & 21 & 3.0 & 12 & 3.6 & 8 & & & & & 4 & 1 \\
\hline 153 & 2.9 & 10 & & & 2.9 & 10 & & & & & & \\
\hline 158 & 2.6 & 22 & 3.2 & 5 & 2.4 & 5 & 28 & 4 & 2.4 & 8 & & \\
\hline 179 & 2.2 & 27 & 14 & 8 & 2.1 & 9 & 3.0 & 10 & & & & \\
\hline 180 & 3.2 & 32 & 3,3 & 10 & 3.1 & 9 & 3.8 & 5 & 2.7 & 7 & 3 & 1 \\
\hline 182 & 3.5 & 2 & 3.5 & 2 & & & & & & & & \\
\hline 183 & 19 & 7 & & & 2.2 & 5 & 10 & 2 & & & & \\
\hline 190 & 2.2 & 33 & 2.5 & 10 & 2.2 & 11 & 2.7 & 3 & 1.9 & 9 & & \\
\hline 191 & 2.4 & 30 & 2,0 & 18 & 2.9 & 10 & 30 & 2 & & & & \\
\hline 193 & 2.6 & 10 & 23 & 8 & 2.7 & 3 & 4.0 & 1 & & & & \\
\hline 194 & 2.7 & 25 & 2,6 & 7 & 3.0 & 10 & 1,8 & 4 & 3.0 & 3 & 3 & 1 \\
\hline 196 & 3.0 & 42 & $\begin{array}{l}3.4 \\
\end{array}$ & 22 & 2.5 & 10 & 3.0 & 1 & 2.8 & 9 & & \\
\hline 197 & 4.0 & 2 & & & & & 4.0 & 2 & & & & \\
\hline 198 & 2.9 & 11 & 3.0 & 10 & & & & & & & 2 & 1 \\
\hline 203 & 2.5 & 22 & 3,4 & 8 & 2.2 & 6 & 13 & 4 & 2.3 & 4 & & $\$$ \\
\hline 204 & 2.5 & 31 & 21 & 9 & 2.3 & 9 & 3.3 & 4 & 2.6 & 8 & 3 & 1 \\
\hline 208 & 28 & 5 & & & 2.3 & 3 & 3.5 & 2 & & & & \\
\hline 209 & 3.0 & 16 & & & 2.6 & 9 & & & 3.6 & 7 & & \\
\hline 210 & 2.7 & 31 & 20 & 7 & 2.7 & 11 & 3.2 & 5 & 2.0 & 7 & 3 & 1 \\
\hline 211 & 1.9 & 38 & 11 & 15 & 2.3 & 12 & 2.8 & 10 & & & 0 & 1 \\
\hline 212 & 3.0 & 1 & & & 3.0 & 1 & & & & & & \\
\hline 213 & 3.2 & 16 & 3.6 & 10 & 1.5 & 2 & 3.0 & 4 & & & & \\
\hline 217 & 2.7 & 7 & 27 & 7 & & & & & & & & \\
\hline 220 & 3.0 & 25 & 3.4 & 9 & 3.3 & 8 & 2.1 & 1 & & & 2 & 1 \\
\hline 221 & 2.4 & 28 & 28 & 13 & 2.4 & 9 & 1,6 & 5 & & & 1 & 1 \\
\hline 224 & 2.3 & 49 & 27 & 15 & 2.0 & 13 & 1.8 & 10 & 2.6 & 11 & & \\
\hline 225 & 1.0 & 29 & 0.5 & $\begin{array}{l}6 \\
6\end{array}$ & 1.9 & 8 & 0,8 & 5 & 0.8 & 9 & 0 & 1 \\
\hline 227 & 3.5 & 2 & & & & & 3.5 & 2 & & & & \\
\hline 230 & 2.7 & 6 & & & 2.7 & 6 & & & & & & \\
\hline
\end{tabular}


Table 5. -Laboratory performance ratings for standard reference water sample T-129 (trace constituents)

-Continued

(MPV,most probable value; ugh, micrograms per liter; mg/L, milligrams per liter; Lab, laboratory number; OLR, overall leboratory rating for all reported values:

V/26, number of reportad valuas of 26 possible values; $R V$, reported value; $<$, less than)

\begin{tabular}{|llll|}
\hline Rating & Absolute Z-value & Rating & Absolute Z-value \\
4 (Excellent) & $0.00-0.50$ & 1 (Questionable) & $1.51-2.00$ \\
3 (Good) & $0.51-1.00$ & O (Poor) & greater than 2.00 \\
2 (Satisfactory) & $1.01-1.50$ & NR (Not Rated) & \\
\hline
\end{tabular}

2 (Satisfactory)

$1.01-1.50$

NR (Not Rated)

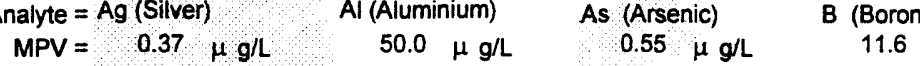

\begin{tabular}{|c|c|c|c|c|c|c|c|c|c|c|c|c|c|c|}
\hline \multicolumn{3}{|c|}{ F-pseudosigma $=$} & \multirow{2}{*}{$\begin{array}{r}140 \\
R V\end{array}$} & \multicolumn{3}{|c|}{11.9} & \multicolumn{2}{|l|}{1.14} & \multicolumn{2}{|l|}{2.8} & \multicolumn{2}{|l|}{1.9} & \multicolumn{2}{|l|}{0.13} \\
\hline Lab & OLR & $V / 26$ & & Rating & RV & Rating & RV & Rating & RV & Rating & $R V$ & Rating & RV & Rating \\
\hline 1 & 3.7 & 22 & 0.11 & 4 & 45.8 & 4 & $<1$ & NR & 8.9 & 3 & 33.0 & 3 & $<0.5$ & NR \\
\hline 3 & 2.6 & 16 & 2,10 & 2 & 59.0 & 3 & $<1$ & NR & 10.0 & 4 & 34.3 & 4 & 0.30 & 2 \\
\hline 4 & 1.2 & 9 & & & & & & & $<10$ & NR & 36.0 & 2 & $<5$ & NR \\
\hline 5 & 3.4 & 13 & $<0.1$ & NR & 48.0 & 4 & $<1$ & NR & & & 32.6 & 3 & $<.5$ & NR \\
\hline 6 & 2.4 & 9 & $<0.5$ & NR & 80.0 & 0 & $<1$ & NR & $<250$ & NR & 34.6 & 4 & $<0.5$ & NR \\
\hline 7 & 2.8 & 14 & $<0.3$ & NR & 56.3 & 3 & $<12$ & NR & & & 33.4 & 4 & $<1$ & NR \\
\hline
\end{tabular}

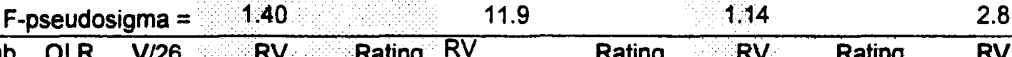

\begin{tabular}{|c|c|c|c|c|c|c|c|c|c|c|c|c|c|c|}
\hline 23 & 2.6 & 14 & 0.03 & 4 & 37.4 & 2 & $<1$ & NR & & & 692 & 0 & $<0.1$ & NR \\
\hline 24 & 3.2 & 24 & 100 & 4 & 81.0 & 0 & 0.50 & 4 & & & 34.3 & 4 & 0.09 & 4 \\
\hline 25 & 1.9 & 10. & $<6$ & NR & $<19$ & NR & $<50$ & NR & $<23$ & NR & 29.0 & 0 & $<2.4$ & NR \\
\hline
\end{tabular}

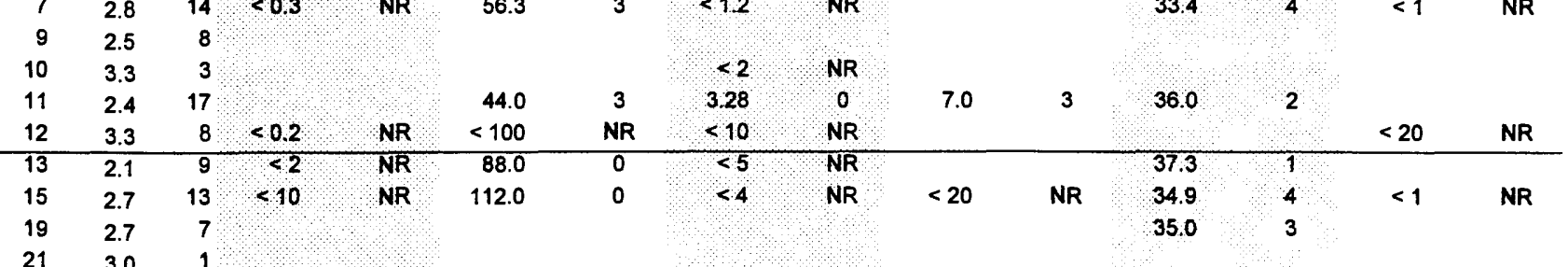

\begin{tabular}{|c|c|c|c|c|c|c|c|c|c|c|c|c|c|c|}
\hline 30 & 3.6 & 9 & & & & & & & & & 35.5 & 3 & & \\
\hline 32 & 2.4 & 16 & $<01$ & NR & 40.5 & 3 & $<0.2$ & NR & 7.0 & 3 & 29.2 & 0 & $<0.2$ & NR \\
\hline
\end{tabular}

$\begin{array}{lllllllll}26 & 2.0 & 3 & <0.3 \quad \text { NR } & <1 & \end{array}$

\begin{tabular}{|c|c|c|c|c|c|c|c|c|c|c|c|c|c|c|}
\hline $\begin{array}{l}36 \\
39\end{array}$ & $\begin{array}{l}2.3 \\
3.8\end{array}$ & $\begin{array}{l}21 \\
13\end{array}$ & $\begin{array}{l}0.00 \\
0.10\end{array}$ & $\begin{array}{l}\mathrm{nr} \\
4\end{array}$ & 75.4 & 0 & $\begin{array}{r}020 \\
<1\end{array}$ & $\begin{array}{c}4 \\
N R\end{array}$ & $\begin{array}{r}167.0 \\
<10\end{array}$ & $\begin{array}{c}0 \\
N R\end{array}$ & $\begin{array}{r}32.1 \\
34.0\end{array}$ & $\begin{array}{l}3 \\
4\end{array}$ & $\begin{array}{r}0.04 \\
<1\end{array}$ & $\begin{array}{c}3 \\
\text { NR }\end{array}$ \\
\hline 43 & 3.8 & 6 & & & & & & & & & & & & \\
\hline 45 & 2.8 & 12 & $<2$ & NR & 38.1 & 3 & $<3$ & NR & & & 34.4 & 4 & & \\
\hline 46 & 3.7 & 11 & & & 48.2 & 4 & 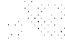 & & 11.6 & 4 & 34.0 & 4 & & \\
\hline 48 & 2.7 & 12 & $<0.2$ & NR & 49.5 & 4 & $<1$ & NR & $<10$ & NR & 47.0 & 0 & $<0.2$ & NR \\
\hline 50 & 2.0 & 4 & $<2$ & NR & 63.0 & 2 & $<1$ & $\mathrm{NR}$ & & & $<50$ & NR & & \\
\hline 51 & 2.3 & 15 & & & & & 0.20 & 4 & & & & & & \\
\hline 52 & 2.6 & 16 & $<1$ & NR & 48.4 & 4 & $<2$ & NR & $<100$ & NR & 31.5 & 2 & $<0.2$ & NR \\
\hline 54 & 2.8 & 4 & & & & & & & & & & & & \\
\hline
\end{tabular}

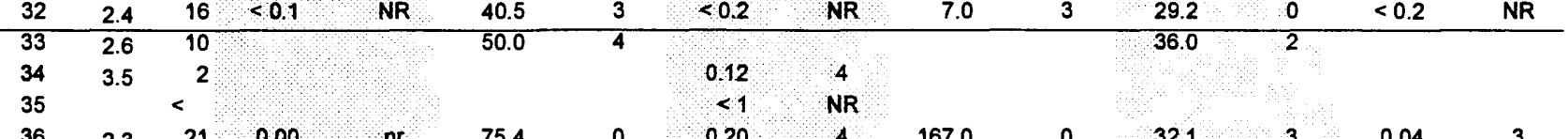

$\begin{array}{llllll}55 & 2.6 & 9 & & 2.8 & 32\end{array}$

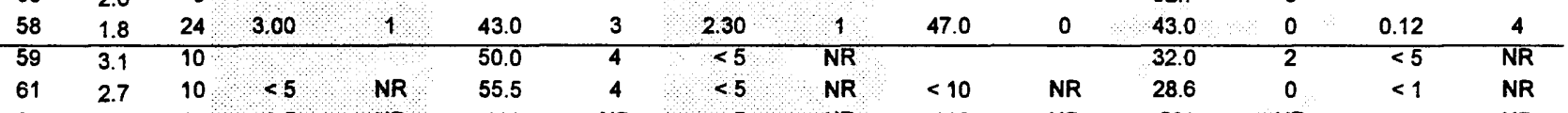

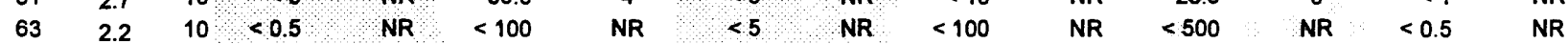

\begin{tabular}{|c|c|c|c|c|c|c|c|c|c|c|c|c|c|c|}
\hline 68 & 2.8 & 16 & 5.50 & 0 & 265.0 & 0 & 0.50 & 4 & & & 31.5 & 2 & $<1$ & NR \\
\hline 69 & 2.3 & 6 & $<2$ & NR & $<100$ & NR & $<5$ & NR & & & & & $<1$ & NR \\
\hline 70 & 3.4 & 9 & $<5$ & NR & $<100$ & NR & $<5$ & NR & $<50$ & NR & 34.6 & 4 & $<2$ & NR \\
\hline 72 & 0.7 & 7 & $<1$ & NR & & & $<1$ & NR & & & 74.3 & 0 & $<1$ & NR \\
\hline 73 & 3.0 & 5 & & & 36.0 & 2 & & & & & & & & \\
\hline 75 & 3.1 & 11 & $<0.1$ & NR & 41.8 & 3 & $<50$ & NR & & & 32.2 & 3 & $<1$ & NR \\
\hline 78 & 3.2 & 21 & 0.60 & 4 & 47.3 & 4 & 1.10 & 4 & & & 42.7 & 0 & 0.10 & 4 \\
\hline 79 & 1.7 & 6 & 0.30 & 4 & & & & & & & & & $<5$ & NR \\
\hline 84 & 3.3 & 4 & & & & & & & & & & & & \\
\hline 85 & 3.3 & 11 & $<5$ & NR & $<100$ & NR & $<2$ & NR & 12.0 & 4 & 32.8 & 3 & $<10$ & NR \\
\hline 86 & 3.5 & 11 & & & 50.1 & 4 & & & 13.5 & 4 & 33.3 & 4 & & \\
\hline 87 & 1.9 & 15 & 2.00 & 2 & & & 2.00 & 3 & & & 112.0 & 0 & & \\
\hline 90 & 1.4 & 12 & 0,32 & 4 & 373.0 & 0 & 780 & 0 & & & 50.3 & 0 & & \\
\hline 91 & 4.0 & 1 & & & & & & & & & & & & \\
\hline 92 & 1.5 & 13 & & & & & & & & & & & & \\
\hline 94 & 3.1 & 11 & 3.00 & 1 & 54.0 & 4 & $<5$ & NR & $<10$ & NR & 34.0 & 4 & $<1$ & NR \\
\hline
\end{tabular}

$\begin{array}{lll}64 & 3.8 & 4 \\ 68 & 2.8 & 16\end{array}$


Table 5. -Laboratory performance ratings for standard reference water sample T-129 (trace constituents) -Continued

Analyte $=\mathrm{Ag}$ (Silver) MPV $=0.37 \mu \mathrm{g} / \mathrm{L}$

Al (Aluminium) As (Arsenic) B (Boron) $50.0 \mu \mathrm{g} / \mathrm{L} \quad 0.55 \mu \mathrm{g} / \mathrm{L} \quad 11.6 \mu \mathrm{g} / \mathrm{L}$

Ba (Barium)

F-pseudosigma $11.9 \mathrm{H} \quad 1.14$

1.14 H.8

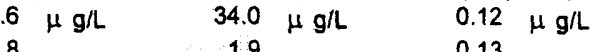

\begin{tabular}{rrrrrrrrrrr} 
Lab & OLR & V/26 & RV & Rating RV & Rating & RV & Rating & RV & Rating \\
\hline 96 & 3.0 & 2 & $<5$ & NR & & & $<5$ & NR & &
\end{tabular}

$100 \quad 2.4$

$101 \quad 2.2$

17

$\begin{array}{rrrr}49.5 & 4 & <0.36 & N R \\ <40 & N R & <2\end{array} \quad N R$

215.0

$\begin{array}{lllllllllll}102 & 2.4 & 14 & <1 & \text { NR } & 39.0 & 3 & <5 & \text { NR } & & \\ 103 & 3.4 & 14 & <5 & \text { NR } & 50.0 & 4 & & & 6.0 & 2\end{array}$

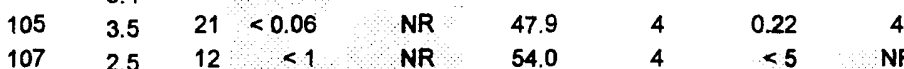

$108 \quad 3.8$

$\begin{array}{lll}109 & 3.3 & 13 \\ 111 & 1.8 & 6\end{array}$

\begin{tabular}{|c|c|c|c|c|c|c|c|c|c|c|c|}
\hline 1.8 & 6 & & & 41.6 & 3 & & & & & & \\
\hline 2.3 & 14 & $<0.5$ & NR & 44.6 & 4 & $<1.5$ & NR & 37.0 & 1 & $<0.2$ & NR \\
\hline 1.5 & 12 & $<5$ & NR & 94.0 & 0 & 0.50 & 4 & & & 1.50 & 0 \\
\hline
\end{tabular}

\begin{tabular}{|c|c|c|c|c|c|c|c|c|c|c|c|c|}
\hline 118 & 1.0 & 1 & $<0.5$ & NR & & $<4$ & NR & & & & & \\
\hline 119 & 3.3 & 15 & & 37.0 & 2 & & 7.0 & 3 & 35.0 & 3 & 0.05 & 3 \\
\hline 121 & 3.4 & 17 & & & & & 13.0 & 4 & 35.0 & 3 & & \\
\hline 122 & 0.0 & 1 & & 94.2 & 0 & & & & & & & \\
\hline
\end{tabular}

$-12$

264.0

2

$\begin{array}{lll}127 & 2.9 & 15 \\ 128 & 2.5 & 11\end{array}$

$129 \quad 0.0$

$133 \quad 3.0 \quad 4 \quad<6 \quad 15$

$134 \quad 3.6 \quad 15<1<1<$

$136 \quad 1.9 \quad 14$

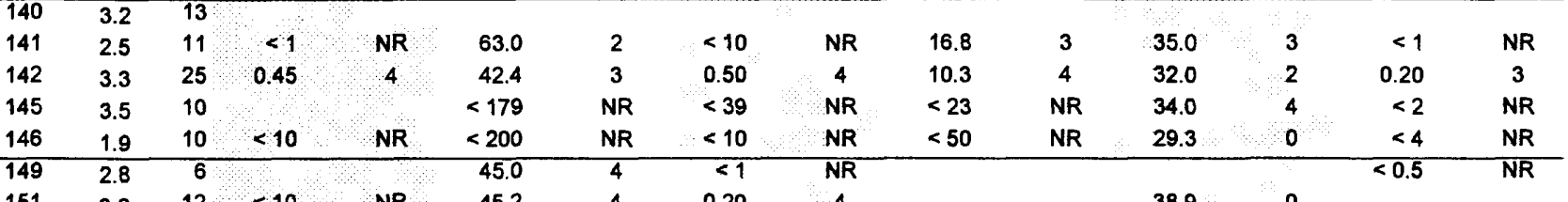

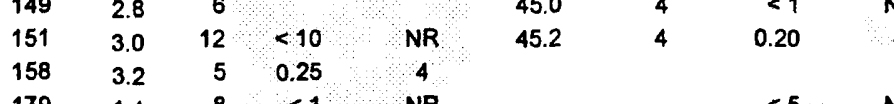

\begin{tabular}{|c|c|c|c|c|c|c|c|c|c|c|c|c|c|}
\hline 179 & 8 & $<1$ & NR & & & $<5$ & NR & & & & & $<.5$ & NR \\
\hline 180 & 10 & $<6.3$ & NR & 53.3 & 4 & $<25.8$ & NR & 16.6 & 3 & 34.5 & 4 & $<.4$ & NR \\
\hline
\end{tabular}

$190 \quad 2.5 \quad 10$

$\begin{array}{llllll}191 & 2.0 & 18 & 18 & 53.40\end{array}$

\begin{tabular}{|c|c|c|c|c|c|c|c|c|c|c|c|c|c|}
\hline 191 & 18 & & & 53.0 & 4 & 82.40 & 0 & & & 32.8 & 3 & & \\
\hline 193 & 6 & $<1$ & NR & & & $<5$ & NR & & & 37.1 & 1 & & \\
\hline $\begin{array}{l}2.0 \\
2.6\end{array}$ & 7 & $<1$ & NR & $<500$ & NR & $<10$ & NR & $<100$ & NR & $<100$ & NR & $<.5$ & NR \\
\hline 3.4 & 22 & 002 & 4 & 514 & 4 & 019 & 4 & & & 347 & 4 & 0.03 & 3 \\
\hline
\end{tabular}

$\begin{array}{rrrrrrrrrrrrrrr}198 & 3.0 & 10<0.001 & \text { NR } & 43.6 & 3 & <0.005 & \text { NR } & 33.8 & 4 & 0.0005 & \text { NR } \\ 203 & 3.4 & 8 & <2 & \text { NR } & 45.0 & 4 & <5 & \text { NR } & 36.6 & 2 & & 4 & \end{array}$

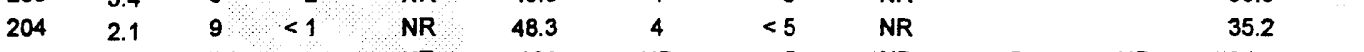

\begin{tabular}{|c|c|c|c|c|c|c|c|c|c|c|c|c|c|c|}
\hline 210 & 2.9 & 7 & $<2$ & NR & $<300$ & NR & $<5$ & NR & $<50$ & NR & 34.6 & 4 & $<0.5$ & NR \\
\hline 211 & 1.1 & 15 & $<0.7$ & NR & 310.0 & 0 & $<2$ & NR & 30.0 & 0 & 30.0 & 0 & $<2$ & NR \\
\hline 213 & 3.6 & 10 & 0.37 & 4 & & & 1.50 & 3 & & & & & 0.12 & 4 \\
\hline 217 & 2.7 & 7 & $<1$ & NR & $<100$ & NR & $<1$ & NR & $<100$ & NR & 34.7 & 4 & $<2$ & NR \\
\hline
\end{tabular}

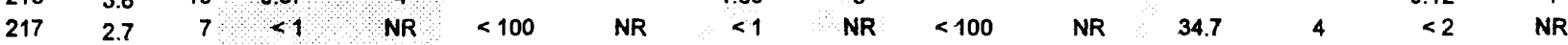

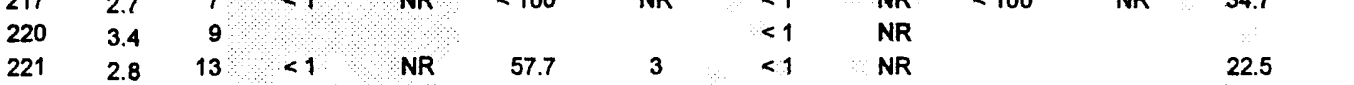

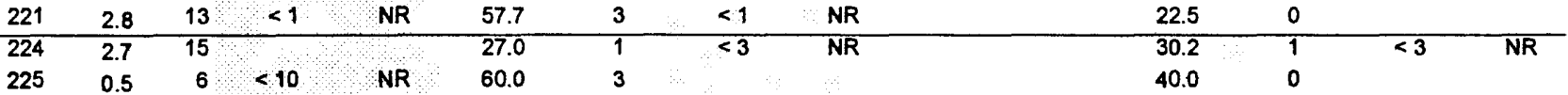


Table 5. -Laboratory performance ratings for standard reference water sample $T-129$ (trace constituents)

--Continued

(MPV,most probable value; ug/h, micrograms per liter; mg/L, milligrams per liter; Lab, laboratory number; OLR, overall laboratory rating for all raported values;

V/26, number of reported values of 26 possible values; RV, reported value; $<$, less than)

\begin{tabular}{|c|c|c|c|}
\hline Rating & Absolute Z-value & Rating & Absolute Z-value \\
\hline 4 (Excellent) & $0.00-0.50$ & 1 (Questionable) & $1.51-2.00$ \\
\hline 3 (Good) & $0.51-1.00$ & $O$ (Poor) & greater than 2.00 \\
\hline 2 (Satisfactory) & $1.01-1.50$ & NR (Not Rated) & \\
\hline $\mathrm{Ca}$ (Calcium) & Cd (Cadmium) & Co (Cobalt) & $\mathrm{Cr}$ (Chromium) \\
\hline $21.1 \mathrm{~m} \mathrm{~g} / \mathrm{L}$ & $0.34 \mu \mathrm{g} / \mathrm{L}$ & $0.74 \mu \mathrm{g} / \mathrm{L}$ & $0.68 \mu \mathrm{g} / \mathrm{L}$ \\
\hline
\end{tabular}

$\begin{aligned} \text { Analyte } & =\mathrm{Ca}(\text { Calcium }) \\ \text { MPV } & =21.1 \mathrm{mg} / \mathrm{L}\end{aligned}$

F-pseudosigma $=$

$0.15 \mu / 20$

Cu (Copper)

$2.7+\mathrm{g} / \mathrm{L}$ 1.4
Fe (Iron)

$10.4 \mu \mathrm{g} / \mathrm{L}$

K (Potassium)

$3.00 \mathrm{~m} \mathrm{~g} / \mathrm{L}$

\begin{tabular}{|c|c|c|c|c|c|c|c|c|c|c|c|c|c|c|}
\hline Lab & $\mathrm{RV}$ & Rating & RV & Rating & RV & Rating & RV & Rating & RV & Rating & RV & Rating & RV & Rating \\
\hline 1 & 21.3 & 4 & 0.32 & 4 & 0.12 & 3 & 0.60 & 4 & 2.7 & 4 & 5.1 & 3 & 2.92 & 4 \\
\hline 3 & 22.5 & 2 & 0.41 & 4 & $<5$ & NR & $<1$ & NR & $<1$ & NR & $<10$ & NR & 4.00 & 0 \\
\hline 4 & 24.0 & 0 & $<10$ & NR & $<10$ & NR & $<15$ & NR & $<6$ & NR & $<30$ & NR & & \\
\hline 5 & 207 & 4 & 0.30 & 4 & $<3$ & NR & $<10$ & NR & $<5$ & NR & & & 321 & 2 \\
\hline 6 & & & 0.25 & 3 & & & 0.52 & 4 & $<50$ & NR & & & & \\
\hline 7 & 22.1 & 2 & $<0.1$ & NR & $<5$ & NR & $<5$ & NR & 3.0 & 4 & 14.9 & 3 & 3.05 & 4 \\
\hline 9 & 19.4 & 1 & & & & & & & 1.7 & 2 & & & 320 & 3 \\
\hline 10 & & & $<1$ & NR & & & $<2$ & NR & 2.0 & 3 & $<20$ & NR & & \\
\hline 11 & 23.3 & 0 & 0.40 & 4 & & & & & & & 6.0 & 3 & 3.05 & 4 \\
\hline 12 & 21.0 & 4 & 0.30 & 4 & & & $<20$ & NR & 2.0 & 3 & $<50$ & NR & 3.00 & 4 \\
\hline 13 & 20.3 & 3 & $<1$ & NR & & & $<5$ & NR & $<20$ & NR & $<20$ & NR & 3.16 & 3 \\
\hline 15 & 227 & 1 & $<10$ & NR & $<20$ & NR & $<1$ & NR & 2.0 & 3 & $<30$ & NR & 2.93 & 4 \\
\hline 19 & 224 & 2 & & & & & & & 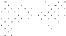 & 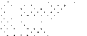 & & & 2.88 & 3 \\
\hline
\end{tabular}

\begin{tabular}{|c|c|c|c|c|c|c|c|c|c|c|c|c|c|c|}
\hline $\begin{array}{l}21 \\
23\end{array}$ & 20.6 & 3 & 0.32 & 4 & & & 0.54 & 4 & 2.1 & 3 & 5.0 & 3 & 2.74 & 2 \\
\hline 24 & 20.9 & 4 & 0.50 & 2 & 0.70 & 4 & 0.90 & 4 & 3.3 & 3 & 5.6 & 3 & 2.91 & 4 \\
\hline 25 & 20.0 & 2 & $<6$ & NR & $<12$ & NR & $<8$ & NR & $<7$ & NR & $<6$ & NR & 2.79 & 2 \\
\hline 26 & & & $<0.3$ & NR & & & $<5$ & NR & $<5$ & NR & $<20$ & NR & & \\
\hline 30 & & & 0.51 & 2 & 0.21 & 4 & 0.30 & 4 & 2.5 & 4 & & & $\because$ & \\
\hline 32 & 21.1 & 4 & 0.41 & 4 & $<0.1$ & NR & $<0.3$ & NR & 1.5 & 1 & 60.5 & 0 & 2.70 & 1 \\
\hline 33 & 22.3 & 2 & & & & & & & $\mathrm{~s}$ & & 10.0 & 4 & 286 & 3 \\
\hline 34 & & & & & & & & & & & & & & \\
\hline 35 & & & & & & & & & & & $<10$ & NR & & \\
\hline 36 : & 19.3 & 1 & 0.22 & 3 & 0.00 & NR & 0.65 & 4 & 7.0 & 0 & 13.5 & 4 & 2.93 & 4 \\
\hline $39^{\circ}$ & 21.1 & 4 & & & $<0.2$ & NR & & & 3.0 & 4 & & & & \\
\hline 43 & 21.0 & 4 & & & & & & & & & $<10$ & NR & 3.00 & 4 \\
\hline 45 & 21.6 & 3 & 0.40 & 4 & & & $<5$ & NR & & & & & 2.96 & 4 \\
\hline 46 & 21.0 & 4 & 0.34 & 4 & & $\therefore$ & & & & & & & 2.95 & 4 \\
\hline 48 & 224 & 2 & 0.30 & 4 & $<50$ & NR & $<1$ & NR & 2.2 & 3 & $<30$ & NR & 3.18 & 3 \\
\hline 50 & & & $<2$ & NR & $<2$ & NR & $<2$ & NR & $<2$ & NR & $<2$ & NR & & 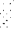 \\
\hline 51 & 20.2 & 3 & 0.30 & 4 & 210 & 2 & 0.10 & 4 & 2.7 & 0 & & & 3.48 & 0 \\
\hline 52 & 20.5 & 3 & 0.30 & 4 & $<1$ & NR & 0.76 & 4 & 13 & 1 & 18.8 & 2 & 2.88 & 3 \\
\hline 54 & 21.5 & 4 & & & & & & & & & & & 1.23 & 0 \\
\hline
\end{tabular}

\begin{tabular}{|c|c|c|c|c|c|c|c|c|c|c|c|c|c|c|}
\hline 58 & 16.9 & 0 & 0.20 & 3 & 0.15 & 4 & 0.40 & 4 & 2.0 & 3 & 30.0 & 0 & 3.39 & 1 \\
\hline 59 & 210 & 4 & $<5$ & NR & & & $<5$ & NR & 8.0 & 0 & $<5$ & NR & 2.95 & 4 \\
\hline 61 & 20.4 & 3 & $<4$ & NR & $<11$ & NR & $<9$ & NR & $<5$ & NR & $<30$ & NR & 3.13 & 3 \\
\hline 63 & 20.1 & 2 & 0.70 & 0 & $<10$ & NR & $<5$ & NR & $<2$ & NR & $<10$ & NR & 3.14 & 3 \\
\hline 64 & 20.8 & 4 & & & & & & & & & & & 3.01 & 4 \\
\hline 68 & 21.5 & 4 & $<2$ & NR & $<5$ & NR & $<5$ & NR & 4.0 & 1 & $<10$ & NR & 2.95 & 4 \\
\hline 69 & 20.7 & 4 & $<1$ & NR & & & $<5$ & NR & $<50$ & NR & $<50$ & NR & 3.35 & 1 \\
\hline 70 & 22.3 & 2 & $<1$ & NR & $<50$ & NR & $<1$ & NR & $<10$ & NR & $<20$ & NR & 3.15 & 3 \\
\hline 72 & 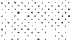 & & $<1$ & NR & 1.40 & 3 & $<1$ & NR & 4.7 & 0 & & & & 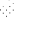 \\
\hline 73 & & & & & & & 3.20 & 1 & 2.4 & 4 & & & & \\
\hline 75 & 21.8 & 3 & 0.26 & 3 & $<5$ & NR & $<2$ & NR & $<3$ & NR & $<5$ & NR & 2.88 & 3 \\
\hline 78 & 20.3 & 3 & 0.40 & 4 & & & 0.60 & 4 & 2.1 & 3 & 7.8 & 4 & 3.08 & 4 \\
\hline 79 & & & $<5$ & NR & & & $<5$ & NR & 6.1 & 0 & & & & \\
\hline 84 & 21.2 & 4 & & & & & & & 3.5 & 2 & & & & \\
\hline 85 & 20.6 & 3 & $<5$ & NR & $<10$ & NR & $<10$ & NR & $<5$ & NR & $<10$ & NR & 3.52 & 0 \\
\hline 86 & 21,3 & 4 & & & & & & & & & 20.9 & 2 & 3.00 & 4 \\
\hline 87 & 200 & 2 & 2.00 & 0 & & & 15.00 & 0 & 5.0 & 0 & $<40$ & NR & 2.98 & 4 \\
\hline 90 & & & 0.36 & 4 & & & 0.09 & 4 & & & & & & \\
\hline 91 & & & & & & & & & & & $<20$ & NR & & \\
\hline 92 & 110 & 0 & 1.50 & 0 & 250 & 2 & 10.00 & 0 & 4.0 & 1 & & & 2.90 & 4 \\
\hline 94 & 221 & 2 & $<3$ & NR & $<5$ & NR & $<5$ & NR & $<5$ & NR & $<30$ & NR & 3.00 & 4 \\
\hline
\end{tabular}


Table 5. -Laboratory performance ratings for standard reference water sample T-129 (trace constituents) -Continued

Analyte $=\mathrm{Ca}$ (Calcium)

$M P V=21.1 \mathrm{mg} / \mathrm{L}$

Cd (Cadmium) $0.34 \mu \mathrm{g} / \mathrm{L}$
Cr (Chromium)

$0.68 \mu \mathrm{g} / \mathrm{L}$
Cu (Copper)
$2.7 \mu \mathrm{g} / \mathrm{L}$

1.4
Fe (Iron)

$10.4 \mu \mathrm{g} / \mathrm{L}$
K (Potassium) $3.00 \mathrm{~m} \mathrm{~g} / \mathrm{L}$ 0.20

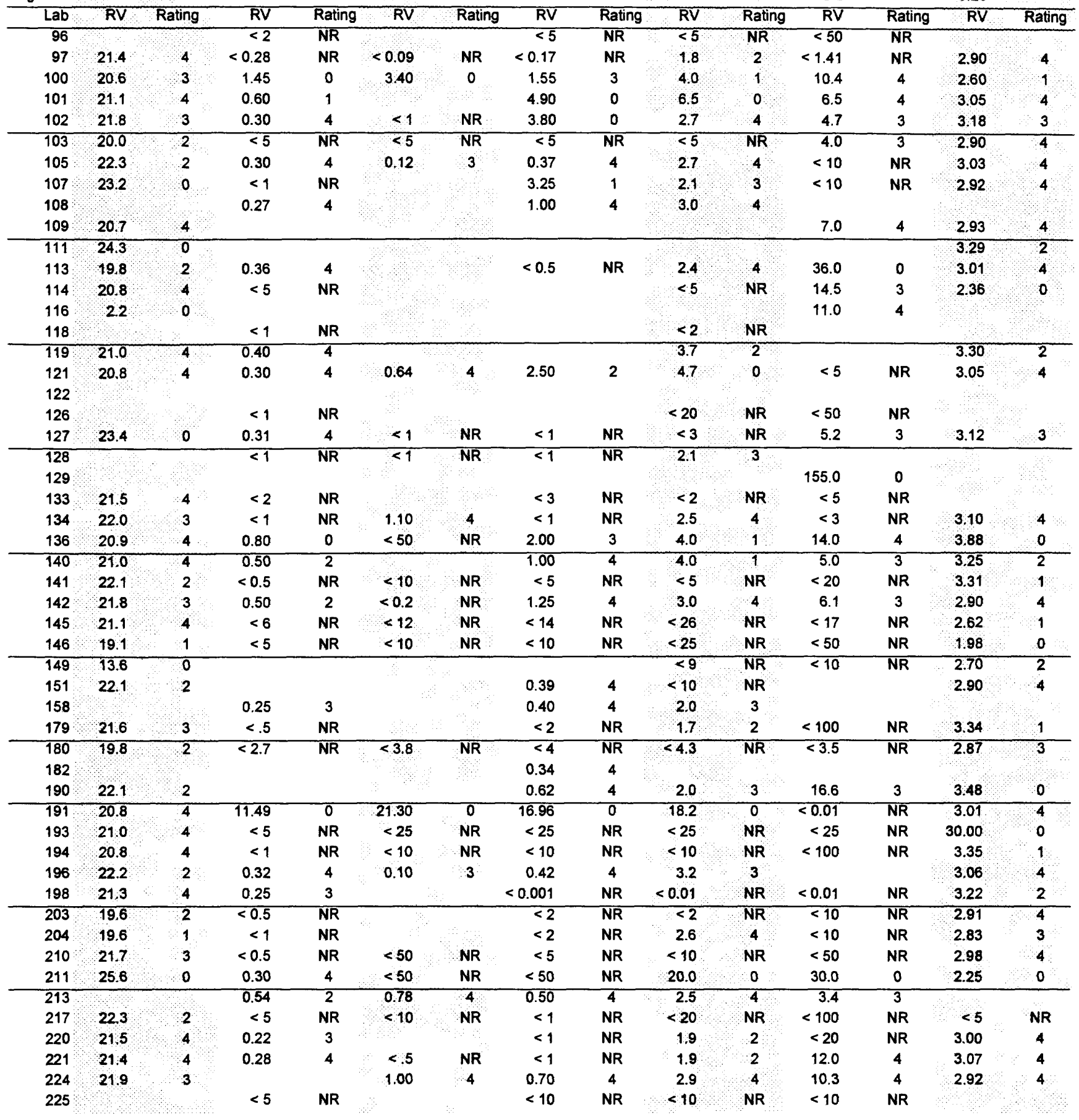


Table 5. - Laboratory performance ratings for standard reference water sample T-129 (trace constituents)

--Continued

(MPV,most probable value; ug/L, micrograms per liter; $m g / L$, milligrams per liter; Lab, laboratory number; OLR, overall laboratory rating for all reported values; V/26, number of reported values of 26 possible values; $R V$, reported value; <, less than)

\begin{tabular}{|llll|}
\hline Rating & Absolute Z-value & Rating & Absolute Z-value \\
4 (Excellent) & $0.00-0.50$ & 1 (Questionable) & $1.51-2.00$ \\
3 (Good) & $0.51-1.00$ & $O$ (Poor) & greater than 2.00 \\
2 (Satisfactory) & $1.01-1.50$ & NR (Not Rated) & \\
\hline
\end{tabular}

Analyte $=\mathrm{Li}$ (Lithium) Mg (Magnesium) Mn (Manganese) Mo (Molybdenum) Na $5.83 \mathrm{~m} \mathrm{~g} / \mathrm{L} \quad 25.2 \mu \mathrm{g} / \mathrm{L} \quad 20.3 \mu \mathrm{g} / \mathrm{L}$

Na (Sodium)

$\mathrm{Ni}$ (Nickel)

$35.5 \mathrm{~m} \mathrm{~g} / \mathrm{L} \quad 1.7 \mu \mathrm{g} / \mathrm{L}$

$\mathrm{Pb}$ (Lead)

$\begin{array}{llllll}0.25 & 2.2 & 2.1 & 1.5 & 1.7 & 1.37\end{array}$

\begin{tabular}{|c|c|c|c|c|c|c|c|c|c|c|c|c|c|c|}
\hline Lab & $\mathrm{RV}$ & Rating & RV & Rating & $\mathrm{RV}$ & Rating & RV & Rating & RV & Rating & RV & Rating & RV & Rating \\
\hline 1 & 17.0 & 4 & 5.92 & 4 & 24.2 & 4 & 19.2 & 3 & 35.0 & 4 & 1.6 & 4 & 0.03 & 3 \\
\hline 3 & 20.0 & 3 & 6.20 & 2 & 25.6 & 4 & 18.0 & 2 & 29.2 & 0 & $<5$ & NR & $<1$ & NR \\
\hline 4 & 14:0 & 1 & 6.20 & 2 & $27: 0$ & 3 & $<50$ & NR & 40.0 & 0 & $<30$ & NR & $<60$ & NR \\
\hline 5 & 17.5 & 4 & 5.82 & 4 & 25.5 & 4 & 13.3 & 0 & 36.0 & 4 & & & $<1$ & NR \\
\hline 6 & & & & & 26.7 & 3 & 24.5 & 1 & & & 0.9 & 4 & $<1$ & NR \\
\hline 7 & & & 6.20 & 2 & 26.7 & 3 & 25.4 & 0 & 37.6 & 2 & $<15$ & NR & $<1$ & NR \\
\hline 9 & & & 5.58 & 3 & 27.3 & 3 & & & 36.0 & 4 & & & & \\
\hline 10 & & & & & 27.0 & 3 & & & & & & & $<2$ & NR \\
\hline 11 & & & 6.25 & 1 & 25.0 & 4 & 16.0 & 0 & 37.2 & 2 & & & 0.40 & 4 \\
\hline 12 & & & 6.00 & 3 & 20.0 & 0 & $<30$ & NR & 35.0 & 4 & $<20$ & NR & $<10$ & NR \\
\hline 13 & & & 4.59 & 0 & 32.0 & 0 & & & 36.2 & 4 & $<20$ & NR & $<5$ & NR \\
\hline 15 & 29.2 & 0 & 5.86 & 4 & 26.0 & 4 & 22.3 & 3 & 36.6 & 3 & $<5$ & NR & $<1$ & NR \\
\hline 19 & & & 6.22 & 1 & 26.0 & 4 & & & 36.0 & 4 & & & & \\
\hline \multicolumn{15}{|l|}{21} \\
\hline 23 & & & 27.00 & 0 & & & 24.0 & 1 & 34.6 & 3 & & & $<0.5$ & NR \\
\hline 24 & 20.0 & 3 & 5.81 & 4 & 26.2 & 4 & 20.0 & 4 & 34.5 & 3 & 4.2 & 2 & 0.20 & 3 \\
\hline 25 & 15.0 & 2 & 5.60 & 3 & 18.0 & 0 & & & 33.9 & 2 & $<49$ & NR & $<71$ & NR \\
\hline 26 & & & & & 26.0 & 4 & & & & & $<5$ & NR & $<5$ & NR \\
\hline 30 & & & & & 23.2 & 3 & 20.3 & 4 & & $\%$ & 1.7 & 4 & & \\
\hline 32 & 15.6 & 3 & 5.68 & 3 & 22.4 & 2 & 17.5 & 2 & 35.0 & 4 & $<0.8$ & NR & $<0.1$ & NR \\
\hline 33 & & & 5.81 & 4 & 20.0 & 0 & & & 34.1 & 3 & & & & \\
\hline \multicolumn{15}{|l|}{34} \\
\hline \multicolumn{15}{|l|}{35} \\
\hline 36 & & & 6.06 & 3 & 37.1 & 0 & 18.1 & 2 & 37.4 & 2 & 3.3 & 3 & 0.55 & 4 \\
\hline 39 & 19.0 & 4 & 6.01 & 3 & 24.0 & 3 & 21.0 & 4 & 35.5 & 4 & & & & \\
\hline 43 & & & 5.80 & 4 & 26.0 & 4 & & & 35.3 & 4 & & & & \\
\hline 45 & & & 5.80 & 4 & 31.5 & 0 & 27.0 & 0 & 36.7 & 3 & & & $<2$ & NR \\
\hline 46 & & & 5.96 & 3 & 24.7 & 4 & & & 36.6 & 3 & & & & \\
\hline 48 & & & 6.17 & 2 & 30.0 & 0 & $<100$ & NR & 34.8 & 4 & 1.6 & 4 & $<1$ & NR \\
\hline 50 & $<50$ & NR & & & 28.0 & 2 & 20.0 & 4 & & & $<2$ & NR & $<2$ & NR \\
\hline 51 & & & 6.00 & 3 & 37.2 & 0 & & & 35.0 & 4 & 2.6 & 3 & 2.00 & 3 \\
\hline 52 & & & 5.76 & 4 & 23.9 & 3 & 20.9 & 4 & 34.0 & 2 & 8.1 & 0 & $<2$ & NR \\
\hline 54 & & & 6.01 & 3 & & & & & 35.4 & 4 & & & & \\
\hline 55 & 22.0 & 1 & 5.69 & 3 & 21.9 & 1 & & & & & & & 1.30 & 4 \\
\hline 58 & & & 4.76 & 0 & 30.0 & 0 & 20.0 & 4 & 70.0 & 0 & 1.0 & 4 & 1.00 & 4 \\
\hline 59 & & & 5.80 & 4 & 24.0 & 3 & & & 36.0 & 4 & $<5$ & NR & $<5$ & NR \\
\hline 61 & & & 5.68 & 3 & 25.0 & 4 & 19.2 & 3 & 34.3 & 3 & $<23$ & NR & $<5$ & NR \\
\hline 63 & 26.0 & 0 & 5.54 & 2 & 26.0 & 4 & 22.0 & 3 & 33.9 & 2 & $<5$ & NR & $<5$ & NR \\
\hline 64 & & & & & & & & & 36.2 & 4 & & & & \\
\hline 68 & 17.0 & 4 & 5.75 & 4 & 25.0 & 4 & 22.0 & 3 & 34.5 & 3 & $<7$ & NR & 1,40 & 4 \\
\hline 69 & $<50$ & NR & 5.45 & 2 & 20.0 & 0 & & & 34.7 & 3 & $<50$ & NR & $<5$ & NR \\
\hline 70 & & & 6.04 & 3 & 25.1 & 4 & $<50$ & NR & 35.8 & 4 & $<20$ & NR & $<1$ & NR \\
\hline 72 & & & & & 47.6 & 0 & 33.6 & 0 & & & 4.2 & 2 & $<2$ & NR \\
\hline 73 & & & & & 26.0 & 4 & & & & & & & & \\
\hline 75 & 15.9 & 3 & 6.10 & 2 & 23.8 & 3 & 20.0 & 4 & 36.8 & 3 & $<5$ & NR & $<3$ & NR \\
\hline 78 & & & 4.30 & 0 & 24.8 & 4 & 21.6 & 3 & 21.9 & 0 & 1.8 & 4 & 2.10 & 3 \\
\hline 79 & & & & & 31.3 & 0 & 20.1 & 4 & 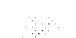 & & $<5$ & NR & & \\
\hline 84 & & & 5.68 & 3 & & & & & 36.1 & 4 & & & & \\
\hline 85 & 19.0 & 4 & 5.78 & 4 & 23.6 & 3 & 21.2 & 4 & 35.0 & 4 & $<10$ & NR & $<50$ & NR \\
\hline 86 & & & 6.08 & 2 & 24.0 & 3 & 21.6 & 3 & 36.0 & 4 & & & & \\
\hline 87 & & & 5.43 & 1 & 24.0 & 3 & 22.5 & 2 & 33.2 & 1 & $<10$ & NR & 20 & NR \\
\hline 90 & & & & & 30.0 & 0 & & & 32.3 & 0 & 1.9 & 4 & 3.26 & 1 \\
\hline 91 & & & & & 25.3 & 4 & & & & & & & & \\
\hline 92 & & & 2.75 & 0 & 24.0 & 3 & & & 34.0 & 2 & 6.0 & 0 & 6.50 & 0 \\
\hline 94 & & & 5.70 & 4 & 25.0 & 4 & 18.0 & 2 & 35.6 & 4 & $<15$ & NR & $<2$ & NR \\
\hline
\end{tabular}


Table 5. - Laboratory performance ratings for standard reference water sample $T-129$ (trace constituents) --Continued

Analyte $=$ Li (Lithium) $M P V=18,0 \mu g / L$

Mg (Magnesium) Mn (Manganese) Mo (Molybdenum) Na (Sodium) $5.83 \mathrm{~m} \mathrm{~g} / \mathrm{L}$ $25.2 \mu \mathrm{g} / \mathrm{L}$ $20.3 \mu \mathrm{g} / \mathrm{L}$ $35.5 \mathrm{~m} \mathrm{~g} / \mathrm{L}$

Ni (Nickel) $\mathrm{Pb}$ (Lead) F-pseudosigma $=$\begin{tabular}{lllllll}
2.6 & 0.25 & 2.2 & 2.1 & 1.5 & 1.7 & $\mu \mathrm{g} / \mathrm{g}$ \\
\hline
\end{tabular}

\begin{tabular}{|c|c|c|c|c|c|c|c|c|c|c|c|c|c|c|}
\hline Lab & RV & Rating & RV & Rating & RV & Rating & RV & Rating & RV & Rating & RV & Ratino & RV & Rating \\
\hline 96 & & & & & 25.6 & 4 & & & & & & & $\frac{k v}{<5}$ & $\frac{\text { Katung }}{\text { NR }}$ \\
\hline 97 & & & 5.80 & 4 & 23.0 & 2 & 20.5 & 4 & 34.9 & 4 & $<0.44$ & NR & 0.66 & 4 \\
\hline 100 & 170 & 4 & 5.90 & 4 & 25.5 & 4 & $<50$ & NR & 35.6 & 4 & 2.2 & 4 & $<2$ & NR \\
\hline 101 & & & 6.00 & 3 & 24.8 & 4 & & & 36.5 & 3 & 0.3 & 3 & 5.20 & 0 \\
\hline 102 & & & 5.00 & 0 & 23.8 & 3 & & & 39.2 & 0 & $<1$ & NR & $<3$ & NR \\
\hline 103 & 16.0 & 3 & 5.70 & 4 & 26.0 & 4 & 20.0 & 4 & 370 & 2 & $<5$ & NR & $<20$ & NR \\
\hline 105 & 18.0 & 4 & 5.87 & 4 & 24.0 & 3 & 20.8 & 4 & 370 & 2 & 1.1 & 4 & 0.10 & 3 \\
\hline 107 & & & 5.79 & 4 & 30.0 & 0 & & & 33.5 & 2 & 1.9 & 4 & $<2$ & NR \\
\hline 108 & & & & & & & & & & & 1.0 & 4 & 0.25 & 3 \\
\hline 109 & 177 & 4 & 6.00 & 3 & 2B. & 2 & 22.0 & 3 & 34,5 & 3 & & & 0.00 & NR \\
\hline 111 & & & 5.99 & 3 & & & & & 34.7 & 3 & & & & \\
\hline 113 & & & 6.04 & 3 & 25.4 & 4 & & & 3.6 & 0 & 1.2 & 4 & $<0.5$ & NR \\
\hline 114 & & & 6.16 & 2 & 28.5 & 2 & & & 44.7 & 0 & & & 5.00 & 0 \\
\hline 116 & & & 5.98 & 3 & 27.0 & 3 & & & 36.0 & 4 & & & & \\
\hline 118 & & & & & & & & & & & $<8$ & NR & $<4$ & NR \\
\hline 119 & & & 5.80 & 4 & 25.0 & 4 & & & 35.3 & 4 & & & & \\
\hline 121 & & & 5.80 & 4 & 26.0 & 4 & 20.0 & 4 & 35.7 & 4 & 4.0 & 2 & 1.20 & 4 \\
\hline \multicolumn{15}{|l|}{122} \\
\hline 126 & & & & & & & & & 35.4 & 4 & & & & \\
\hline 127 & 18.0 & 4 & 5.83 & 4 & 25.1 & 4 & 23.0 & 2 & 36.5 & 3 & $<3$ & NR & $<1$ & NR \\
\hline 128 & 122 & 0 & & & 21.7 & 1 & 19.0 & 3 & & & 1.0 & 4 & $<1$ & NR \\
\hline 129 & & & & & 100 & 0 & & & & & & & 3 & \\
\hline 133 & & & 5.99 & 3 & & & & & & & $<5$ & NR & $<20$ & NR \\
\hline 134 & 22.0 & 1 & 5.78 & 4 & 25.0 & 4 & 20.0 & 4 & 36.0 & 4 & 1.4 & 4 & $<1$ & NR \\
\hline 136 & & & 5.79 & 4 & 34.0 & 0 & & & 37.0 & 2 & 1.0 & 4 & & \\
\hline 140 & & & 5.80 & 4 & 23.0 & 2 & & & 36.5 & 3 & 1.0 & 4 & 0.50 & 4 \\
\hline 141 & & & 6.32 & 1 & 27.0 & 3 & 19.0 & 3 & 37.2 & 2 & $<10$ & NR & $<5$ & NR \\
\hline 142 & 20.0 & 3 & 6.14 & 2 & 25.3 & 4 & 38.5 & 0 & 35.3 & 4 & 1.0 & 4 & 1.00 & 4 \\
\hline 145 & $<20$ & NR & 5.76 & 4 & 24.0 & 3 & 20.0 & 4 & 34.8 & 4 & $<22$ & NR & $<84$ & NR \\
\hline 146 & & & 5.60 & 3 & 23.2 & 3 & 17.8 & 2 & 34.5 & 3 & $<40$ & NR & $<5$ & NR \\
\hline 149 & & & & & 26.0 & 4 & & & 35.0 & 4 & $<1$ & NR & $<2$ & NR \\
\hline 151 & & & 5.85 & 4 & 37.2 & 0 & 18.8 & 3 & 35.0 & 4 & 1.8 & 4 & & 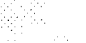 \\
\hline 158 & & & & & & & & & & & & & $<2$ & NR \\
\hline 179 & & & 5.60 & 3 & 18.0 & 0 & 31.7 & 0 & 37.4 & 2 & $<3$ & NR & $<5$ & NR \\
\hline 180 & & & 5.95 & 4 & 24.4 & 4 & 18.7 & 3 & 33.9 & 2 & $<7.3$ & NR & $<32.7$ & NR \\
\hline 182 & & & & & & & 21.5 & 3 & & & & & & \\
\hline 190 & & & 6.15 & 2 & 23.4 & 3 & & & 33.1 & 1 & & & 0.15 & 3 \\
\hline 191 & & & 5.81 & 4 & 24.0 & 3 & & & 36.0 & 4 & 56.2 & 0 & 30.70 & 0 \\
\hline 193 & & & 5.60 & 3 & & & & & 34.0 & 2 & $<25$ & NR & $<5$ & NR \\
\hline 194 & & & 5.50 & 2 & $<50$ & NR & 23.0 & 2 & 35.7 & 4 & $<100$ & NR & $<5$ & NR \\
\hline 196 & 18.8 & 4 & 6.12 & 2 & 28.2 & 2 & 21.0 & 4 & 37.3 & 2 & 1.5 & 4 & 0.06 & 3 \\
\hline 198 & & & 6.04 & 3 & 24.1 & 3 & $<0.05$ & 0 & 36.1 & 4 & $<0.02$ & NR & $<0.005$ & NR \\
\hline 203 & & & 5.72 & 4 & 26.0 & 4 & & & 36.1 & 4 & $<20$ & NR & $<2$ & NR \\
\hline 204 & & & 6.42 & 0 & $<10$ & 0 & & & 33.0 & 1 & & & $<5$ & NR \\
\hline 210 & $<1000$ & NR & 6.20 & 2 & $<50$ & NR & $<50$ & NR & 38.8 & 0 & $<10$ & NR & $<2$ & NR \\
\hline 211 & & & 5.70 & 4 & 40.0 & 0 & $<100$ & NR & 31.1 & 0 & 30.0 & 0 & 1.00 & 4 \\
\hline 213 & & & & & & & & & & & $<2$ & NR & 1.40 & 4 \\
\hline 217 & $<50$ & NR & 6.31 & 1 & 26.5 & 3 & 20.0 & 4 & 36.6 & 3 & $<40$ & NR & $<1$ & NR \\
\hline 220 & & & 5.91 & 4 & 27.0 & 3 & & & 34.1 & 3 & & & & \\
\hline 221 & & & 12.30 & 0 & 23.8 & 3 & 14.8 & 0 & 36.0 & 4 & $<2$ & NR & 1.00 & 4 \\
\hline 224 & & & 6.28 & 1 & 22.0 & 2 & 22.0 & 3 & 34.3 & 3 & & & $<3$ & NR \\
\hline 225 & & & 4.93 & 0 & 20.0 & 0 & $<20$ & NR & & & $<20$ & NR & $<20$ & NR \\
\hline
\end{tabular}


Table 5. - Laboratory performance ratings for standard reference water sample T-129 (trace constituents) --Continued

(MPV,most probable value; uglh, micrograms per liter, mg/L, milligrams per liter; Lab, laboratory number; OLR, overall laboratory rating for all reported values: V/26, number of reported values of 26 possible values; RV, reported value; <. less than)

\begin{tabular}{|llll|}
\hline Rating & Absolute Z-value & Rating & Absolute Z-value \\
4 (Excellent) & $0.00-0.50$ & 1 (Questionable) & $1.51-2.00$ \\
3 (Good) & $0.51-1.00$ & $O$ (Poor) & greater than 2.00 \\
2 (Satisfactory) & $1.01-1.50$ & NR (Not Reted) & \\
\hline
\end{tabular}

Analyte $=\mathrm{Sb}$ (Antimony) $\quad \mathrm{Se}$ (Selenium) SiO2 (Silica) Sr (Strontium) V Nanadium) $\quad \mathrm{Zn}$ (Zinc)

MPV $=\begin{array}{llllllll}0.55 \mu \mathrm{g} / \mathrm{L} & 1.60 \mu \mathrm{g} / \mathrm{L} & 9.15 \mathrm{~m} \mathrm{~g} / \mathrm{L} & 181 \mu \mathrm{g} / \mathrm{L} & 1.0 \mu \mathrm{g} / \mathrm{L} & 72.0 \mu \mathrm{g} / \mathrm{L}\end{array}$

F-pseudosigma $=\begin{array}{llllllll}0.87 & 1.59 & 0.83 & 11 & 2.4 & 4.8\end{array}$

\begin{tabular}{|c|c|c|c|c|c|c|c|c|c|c|c|c|}
\hline Lab & RV & Rating & RV & Rating & $\mathrm{RV}$ & Rating & RV & Rating & RV & Rating & RV & Rating \\
\hline 1 & 0.22 & 4 & $<1$ & NR & 9.31 & 4 & 177 & 4 & $<6$ & NR & 67.7 & 3 \\
\hline 3 & $<2$ & NR & $<1$ & NR & 8.74 & 4 & 188 & 3 & $<5$ & NR & 77.7 & 2 \\
\hline 4 & & & & & 10.00 & 2 & 200 & 1 & $<10$ & NR & 55.0 & 0 \\
\hline 5 & & & $<2$ & NR & 9.60 & 3 & 185 & 4 & & & 72.6 & 4 \\
\hline 6 & $<3$ & NR & 7.65 & 0 & & & & & & & 68.0 & 3 \\
\hline 7 & $<26$ & NR & 1.90 & 4 & 10.26 & 2 & 184 & 4 & $<4$ & NR & 77.1 & 2 \\
\hline 9 & & & & & & & 222 & 0 & & & 70.0 & 4 \\
\hline 10 & & & $<2$ & NR & & & & & & & 70.0 & 4 \\
\hline 11 & & & 2.29 & 4 & 4.18 & 0 & 190 & 3 & & & 75.0 & 3 \\
\hline 12 & $<100$ & NR & $<2$ & NR & & & & & 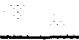 & & 70.0 & 4 \\
\hline 13 & & & $<5$ & NR & 9.18 & 4 & & & & & 72.0 & 4 \\
\hline 15 & $<50$ & NR & $<1$ & NR & 8.46 & 3 & 173 & 3 & $<10$ & NR & 75.4 & 3 \\
\hline 19 & & & & & & & & & & & 77.0 & 2 \\
\hline 21 & & & & & & & & & & & & \\
\hline 23 & $<2$ & NR & $<1$ & NR & 9.61 & 3 & & & 0.9 & 4 & 70.5 & 4 \\
\hline 24 & 1.00 & 3 & 0.40 & 3 & 4.57 & 0 & 178 & 4 & & & 72.5 & 4 \\
\hline 25 & $<51$ & NR & $<129$ & NR & 9.07 & 4 & 176 & 4 & $<3$ & NR & 49.0 & 0 \\
\hline 26 & & $\therefore \quad \therefore$ & $<1$ & NR & & & & & & & 78.0 & 2 \\
\hline 30 & 0.50 & 4 & & & & & & & & & & \\
\hline 32 & $<0.3$ & NR & $<10$ & NR & 8.56 & 3 & 173 & 3 & $<0.2$ & NR & 67.9 & 3 \\
\hline 33 & & & & & 9.61 & 3 & 202 & 1 & & & & \\
\hline 34 & & & 0.15 & 3 & & & & & & & & \\
\hline 35 & & & $<1$ & NR & & & & & & & & \\
\hline 36 & 0.22 & 4 & 1.70 & 4 & 18.00 & 0 & & & & & 64.1 & 1 \\
\hline 39 & 0.20 & 4 & & & 9.12 & 4 & 181 & 4 & $<2$ & NR & 76.0 & 3 \\
\hline 43 & & & & & 9.70 & 3 & & & & & & \\
\hline 45 & $<5$ & NR & 0.36 & 3 & 9.45 & 4 & & & & & 65.2 & 2 \\
\hline 46 & & & & & & & 191 & 3 & & & 72.5 & 4 \\
\hline 48 & $<3$ & NR & $<2$ & NR & & & & & 4.0 & 2 & 70.0 & 4 \\
\hline 50 & & & $<1$ & NR & & . & & & $<5$ & NR & 88.0 & 0 \\
\hline 51 & & & & & 6.90 & 0 & & & 0.3 & 4 & 59.0 & 0 \\
\hline 52 & $<6$ & NR & $<5$ & NR & 6.53 & 0 & 174 & 3 & $<2$ & NR & 66.8 & 2 \\
\hline 54 & & & & & $\therefore$ & & & & & & & \\
\hline 55 & & & & & 9.29 & 4 & 172 & 3 & & & 82.0 & 0 \\
\hline 58 & 11.00 & 0 & 6.00 & 0 & 5.18 & 0 & & & 3.0 & 3 & 70.0 & 4 \\
\hline 59 & & & & & & & 178 & 4 & & & 65.0 & 2 \\
\hline 61 & $<26$ & NR & $<5$ & NR & 4.44 & 0 & & & $<9.1$ & NR & 72.5 & 4 \\
\hline 63 & $<5$ & NR & $<5$ & NR & 8.89 & 4 & & & $<10$ & NR & 66.0 & 2 \\
\hline 64 & & & & & 8.50 & 3 & & & & & & \\
\hline 68 & $<0.5$ & NR & 1.20 & 4 & & & 170 & 3 & $<3$ & NR & 91.0 & 0 \\
\hline 69 & $<5$ & NR & $<5$ & NR & & & & & & & 70.0 & 4 \\
\hline 70 & $<5$ & NR & $<5$ & NR & 9.47 & 4 & 183 & 4 & $<50$ & NR & 74.5 & 3 \\
\hline 72 & & & & & & & & & & & 142.0 & 0 \\
\hline 73 & & & & & & & & & & & 73.0 & 4 \\
\hline 75 & $<50$ & NR & $<1$ & NR & & & & & $<5$ & NR & 71.7 & 4 \\
\hline 78 & 130 & 3 & 1.30 & 4 & 9.42 & 4 & & & $<1$ & NR & 71.0 & 4 \\
\hline 79 & & & 5.20 & 0 & & & & & & & 78.7 & 2 \\
\hline \multicolumn{13}{|l|}{84} \\
\hline 85 & $<100$ & NR & $<2$ & NR & & & 174 & 3 & $<20$ & NR & 74.2 & 4 \\
\hline 86 & & & & & & & & & & & 71.2 & 4 \\
\hline 87 & & & 2.00 & 4 & 9.70 & 3 & & & & & 72.0 & 4 \\
\hline 90 & & & 9.45 & 0 & & & & & & & 62.0 & 0 \\
\hline \multicolumn{13}{|l|}{91} \\
\hline 92 & & & & & 898 & 4 & & & & & 68.0 & 3 \\
\hline 94 & & & $<5$ & NR & & & 177 & 4 & $<5$ & NR & 80.0 & 1 \\
\hline
\end{tabular}


Table 5. - Laboratory performance ratings for standard reference water sample T-129 (trace constituents) --Continued

Analyte $=\mathrm{Sb}$ (Antimony)

MPV $=0.55 \mu \mathrm{g} / \mathrm{L}$

Se (Selenium) $1.60 \mu \mathrm{g} / \mathrm{L}$
$\mathrm{SiO} 2$ (Silica) $9.15 \mathrm{~m} \mathrm{~g} / \mathrm{L}$
Sr (Strontium)

$181 \mu \mathrm{g} / \mathrm{L}$
$\mathrm{V}$ (Vanadium) $10 \mu \mathrm{g} / \mathrm{L}$
Zn (Zinc) $72.0 \mu \mathrm{g} / \mathrm{L}$

F-pseudosigma $=\quad 0.87$ $\begin{array}{lll}0.83 & 11 & 2.4\end{array}$

\begin{tabular}{|c|c|c|c|c|c|c|c|c|c|c|c|c|}
\hline Lab & $\mathbf{R V}$ & Rating & RV & Rating & $\mathbf{R V}$ & Rating & RV & Rating & RV & Rating & RV & Rating \\
\hline 96 & & & $<5$ & NR & & & & & & & 77.0 & 2 \\
\hline 97 & & & $<0.23$ & NR & 10.80 & 1 & 166 & 2 & 7.4 & 0 & 48.0 & 0 \\
\hline 100 & $<2$ & NR & $<2$ & NR & 10.60 & 1 & 165 & 2 & $<10$ & NR & 74.4 & 4 \\
\hline 101 & & & & & 4.53 & 0 & & & & & 76.0 & 3 \\
\hline 102 & $<1$ & NR & $<1$ & NR & 6.98 & 0 & 181 & 4 & $<1$ & NR & 67.8 & 3 \\
\hline 103 & & & & & 9.20 & 4 & 186 & 4 & $<5$ & NR & 73.0 & 4 \\
\hline 105 & 0.23 & 4 & 0.09 & 3 & 10.19 & 2 & 180 & 4 & $<20$ & NR & 74.0 & 4 \\
\hline 107 & & & $<5$ & NR & 9.28 & 4 & & & & & 74.0 & 4 \\
\hline 108 & & & & & & & & & & & 74.0 & 4 \\
\hline 109 & & & 0.40 & 3 & 8.97 & 4 & 201 & 1 & & & & \\
\hline 111 & & & & & 212 & 0 & & & & & & \\
\hline 113 & $\begin{array}{r}<2 \\
\end{array}$ & NR & $<1$ & NR & 10.03 & 2 & 225 & 0 & & & 83.0 & 0 \\
\hline 114 & 179.50 & 0 & & & & & & & & & 67.5 & 3 \\
\hline 116 & & & & & 8.88 & 4 & 181 & 4 & & & 79.0 & 2 \\
\hline 118 & & & $<5$ & NR & & & & & & & 63.0 & 1 \\
\hline 119 & 0.60 & 4 & 0.60 & 3 & 9.81 & 3 & & & & & 73.0 & 4 \\
\hline 121 & & & & & 9.60 & 3 & 181 & 4 & & & 73.0 & 4 \\
\hline \multicolumn{13}{|l|}{122} \\
\hline 126 & & & $<1$ & NR & & & & & & & 71.0 & 4 \\
\hline 127 & $<2$ & NR & $<3$ & NR & 9.09 & 4 & 187 & 3 & $<4$ & NR & 75.4 & 3 \\
\hline 128 & $<1$ & NR & 2.55 & 3 & & & 186 & 4 & $<1$ & NR & 75.0 & 3 \\
\hline \multicolumn{13}{|l|}{129} \\
\hline 133 & & & $<5$ & NR & & & & & & & 66.4 & 2 \\
\hline 134 & & & $<1$ & NR & 9.49 & 4 & 190 & 3 & $<1$ & NR & 73.0 & 4 \\
\hline 136 & & & $<10$ & NR & & & & & $<10$ & NR & 96.0 & 0 \\
\hline 140 & & & & & 9.56 & 4 & & & & & 72.0 & 4 \\
\hline 141 & $<3$ & NR & $<2$ & NR & 8.82 & 4 & & & $<10$ & NR & 68.0 & 3 \\
\hline 142 & 1.50 & 2 & 1.60 & 4 & 8.97 & 4 & 189 & 3 & 1.0 & 4 & 73.6 & 4 \\
\hline 145 & & & & & 8.99 & 4 & 170 & 3 & $<18$ & NR & 74.0 & 4 \\
\hline 146 & $<50$ & NR & $<10$ & NR & 8.32 & 3 & 170 & 3 & $<10$ & NR & 63.6 & 1 \\
\hline 149 & $<3$ & NR & $<2$ & NR & & & 170 & 3 & & & & \\
\hline 151 & & & $<1$ & NR & & & & & & & 69.3 & 3 \\
\hline 158 & & & & & & & & & & & 65.0 & 2 \\
\hline 179 & $<<5$ & NR & $<5$ & NR & & & & & & & 52.0 & 0 \\
\hline 180 & $<27: 1$ & NR & $<45$ & NR & & & & & $<4.7$ & NR & 73.5 & 4 \\
\hline \multicolumn{13}{|l|}{182} \\
\hline 190 & & & & & & & & & & & 73.1 & 4 \\
\hline 191 & & & 11.20 & 0 & 9.53 & 4 & 173 & 3 & & & 69.0 & 3 \\
\hline 193 & & & $<5$ & NR & & & & & & . & 72.0 & 4 \\
\hline 194 & $<5$ & NR & $<5$ & NR & & & 180 & 4 & $<10$ & NR & 80.0 & 1 \\
\hline 196 & $\begin{array}{r}0,23 \\
\end{array}$ & 4 & 0.20 & 3 & & & 191 & 3 & 0.1 & 4 & & \\
\hline 198 & $<0.01$ & NR & $<0.01$ & NR & & 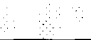 & & & & & 74.4 & 4 \\
\hline 203 & & & $<5$ & NR & & & & & & & 68.0 & 3 \\
\hline 204 & & & $<5$ & NR & & & & & & & 69.0 & 3 \\
\hline 210 & $\begin{array}{r}<5 \\
\end{array}$ & NR & $<5$ & NR & 9.88 & 3 & $<500$ & NR & $<50$ & NR & 74.4 & 4 \\
\hline 211 & $<2$ & NR & $<2$ & NR & 6.28 & 0 & & & $<2$ & NR & 70.0 & 4 \\
\hline 213 & & & & & & & & & & & 70.0 & 4 \\
\hline 217 & $<1$ & NR & $<1$ & NR & & & & & $<10$ & NR & 77.1 & 2 \\
\hline 220 & & & 1.00 & 4 & & & & & & & 72.0 & 4 \\
\hline 221 & & & $<1$ & NR & & & & & & & 74.0 & 4 \\
\hline 224 & & & & & 3.54 & 0 & & & 0.1 & 4 & 65.7 & 2 \\
\hline 225 & & & & & & & 154 & 0 & & & 60.0 & 0 \\
\hline
\end{tabular}


Table 6. - Laboratory performance ratings for standard reference water sample M-130 (major constituents) -Continued

\begin{tabular}{|c|c|c|c|c|c|c|c|c|c|c|c|c|}
\hline \multicolumn{5}{|c|}{ Analyte $=$ Akalinity } & \multicolumn{2}{|c|}{ B (Boron) } & \multicolumn{2}{|l|}{$\mathrm{Ca}$ (Calcium) } & \multicolumn{2}{|l|}{ Cl (Chloride) } & \multicolumn{2}{|l|}{ DSRD } \\
\hline & & MPV $=$ & $60.0 \mathrm{mg} / \mathrm{L}$ & & 8.87 & $\mu \mathrm{g} / \mathrm{L}$ & $212 \mathrm{mg} / \mathrm{L}$ & & $21.4 \mathrm{mg} / \mathrm{L}$ & & $200 \mathrm{mg} / \mathrm{L}$ & \\
\hline & -pseudo & igma $=$ & 19 & & 8.23 & & 1.0 & & 1.9 & & 13 ? & \\
\hline Lab & OLR & V/15 & $\mathrm{RV}$ & Rating & RV & Rating & RV & Rating & RV & Rating & RV & Rating \\
\hline 113 & 2.9 & 14 & 55.9 & 0 & & & 197 & 1 & 20.4 & 3 & 208 & 3 \\
\hline 114 & 2.7 & 11 & 640 & 0 & & & 19.9 & 2 & 21.1 & 4 & 237 & 0 \\
\hline 116 & 3.4 & 6 & 59.9 & 4 & & & 21.4 & 4 & 21.0 & 4 & & \\
\hline 118 & 1.5 & 4 & 617 & 3 & & & & & & & 228 & 0 \\
\hline 119 & 3.3 & 14 & 58.0 & 2 & 10.00 & 4 & 21.0 & 4 & 20.0 & 3 & 200 & 4 \\
\hline 121 & 3.6 & 7 & & & 20.00 & 2 & 208 & 4 & & & & \\
\hline 122 & 2.8 & 11 & 616 & 3 & & & 20.7 & 3 & 18.7 & 2 & 175 & 1 \\
\hline 127 & 3.3 & 14 & 607 & 4 & 9.34 & 4 & 228 & 1 & 21.0 & 4 & 192 & 3 \\
\hline 128 & 2.3 & 12 & $56: 9$ & 1 & 34.60 & 0 & 222 & 2 & 21.4 & 4 & & \\
\hline 129 & 2.6 & 10 & & & & & 192 & 0 & 22.7 & 3 & 225 & 1 \\
\hline 131 & 2.3 & 4 & 570 & 1 & & & & & 20.1 & 3 & & \\
\hline 133 & 2.3 & 4 & 61,3 & 3 & $<20$ & NR & 20.5 & 3 & & & & \\
\hline 134 & 3.6 & 13 & 60.0 & 4 & & & 22.0 & 3 & 21.8 & 4 & 201 & 4 \\
\hline 136 & 2.4 & 10 & 53,5 & 0 & 6.80 & 4 & 20.6 & 3 & 23.0 & 3 & & \\
\hline 138 & 2.9 & 11 & & & & & 22.8 & 1 & 21.4 & 4 & 205 & 4 \\
\hline 140 & 3.1 & 13 & 574 & 2 & 19.60 & 2 & 210 & 4 & 22.2 & 4 & 199 & 4 \\
\hline 141 & 2.6 & 13 & 610 & 3 & 8.05 & 4 & 21.9 & 3 & 20.9 & 4 & 173 & 1 \\
\hline 142 & 3.1 & 12 & & & & & 22,4 & 2 & 22.8 & 3 & 198 & 4 \\
\hline 143 & 3.2 & 5 & 570 & 1 & $<23$ & NR & & & 22.6 & 3 & & \\
\hline 145 & 2.8 & 12 & 59,0 & 3 & $<50$ & NR & 21.0 & 4 & 23.5 & 2 & & \\
\hline 146 & 1.2 & 10 & & & & & 18.6 & 0 & 25.5 & 0 & 190 & 3 \\
\hline 149 & 2.5 & 4 & 61,0 & 3 & & & 23.6 & 0 & & & & \\
\hline 151 & 3.6 & 8 & 60.4 & 4 & & & 210 & 4 & & & & \\
\hline 153 & 2.9 & 10 & 59,0 & 3 & & & 23.0 & 1 & 19.8 & 3 & & \\
\hline 156 & 2.4 & 5 & & & & & & & 21.1 & 4 & 218 & 2 \\
\hline 179 & 2.1 & 9 & 610 & 3 & $<11.6$ & NR & 228 & 1 & 20.0 & 3 & & \\
\hline 180 & 3.1 & 9 & & & & & 20.3 & 3 & 20.2 & 3 & & \\
\hline 183 & 2.2 & 5 & 59,3 & 4 & & & & & 23.5 & 2 & & \\
\hline 190 & 2.2 & 11 & 711 & 0 & & & 24.6 & 0 & 22.1 & 4 & 205 & 4 \\
\hline 191 & 2.9 & 10 & & & & & 201 & 2 & 22.0 & 4 & & \\
\hline 193 & 2.7 & 3 & & & $<100$ & NR & & & 0.0 & 0 & & \\
\hline 194 & 3.0 & 10 & & & & & 21,6 & 4 & 20.5 & 4 & 199 & 4 \\
\hline 196 & 2.5 & 10 & 533 & 0 & & & 217 & 3 & 22.4 & 3 & & \\
\hline 203 & 2.2 & 6 & 560 & 0 & & & & & 20.6 & 4 & & \\
\hline 204 & 2.3 & 9 & & & & & 210 & 3 & 19.3 & 2 & & \\
\hline 208 & 2.3 & 3 & & & & & & & 20.1 & 3 & & \\
\hline 209 & 2.6 & 9 & 61,0 & 3 & $<50$ & NR & 217 & 4 & 20.9 & 4 & & \\
\hline 210 & 2.7 & 11 & 60.8 & 4 & $<40$ & NR & 22.0 & 3 & 21.8 & 4 & & \\
\hline 211 & 2.3 & 12 & & & & & 20.3 & 3 & 21.7 & 4 & 212 & 3 \\
\hline 212 & 3.0 & 1 & 59,0 & 3 & & & & & & & & \\
\hline 213 & 1.5 & 2 & & & & & & & 17.5 & 0 & & \\
\hline 220 & 3.3 & 8 & & & & & 21.2 & 4 & 23.3 & 2 & & \\
\hline 221 & 2.4 & 9 & 60.0 & 4 & & & 21.5 & 4 & 19.8 & 3 & 228 & 0 \\
\hline 224 & 2.0 & 13 & 58,0 & 2 & & & 213 & 4 & 22.1 & 4 & 237 & 0 \\
\hline 225 & 1.9 & 8 & & & & & 210 & 4 & 24.0 & 2 & & \\
\hline 230 & 2.7 & 6 & & & & & 21.4 & 4 & 22.1 & 4 & & \\
\hline
\end{tabular}


Table 6. -Laboratory performance ratings for standard reference water sample $M-130$ (major constituents)

--Continued

(MPV,most probable value; ug/L, micrograms per liter; mg/L, milligrams per liter; Lab, laboratory number; OLR, overall laboratory rating for all reported values; V/15, number of reported values of 16 possible values; $R V$, reported value; <, less than)

\begin{tabular}{|llll|}
\hline Rating & Absolute Z-value & Rating & Absolute Z-value \\
4 (Excellent) & $0.00-0.50$ & 1 (Questionable) & $1.51-2.00$ \\
3 (Good) & $0.51-1.00$ & O (Poor) & greater than 2.00 \\
2 (Satisfactory) & $1.01-1.50$ & NR (Not Rated) & \\
\hline
\end{tabular}

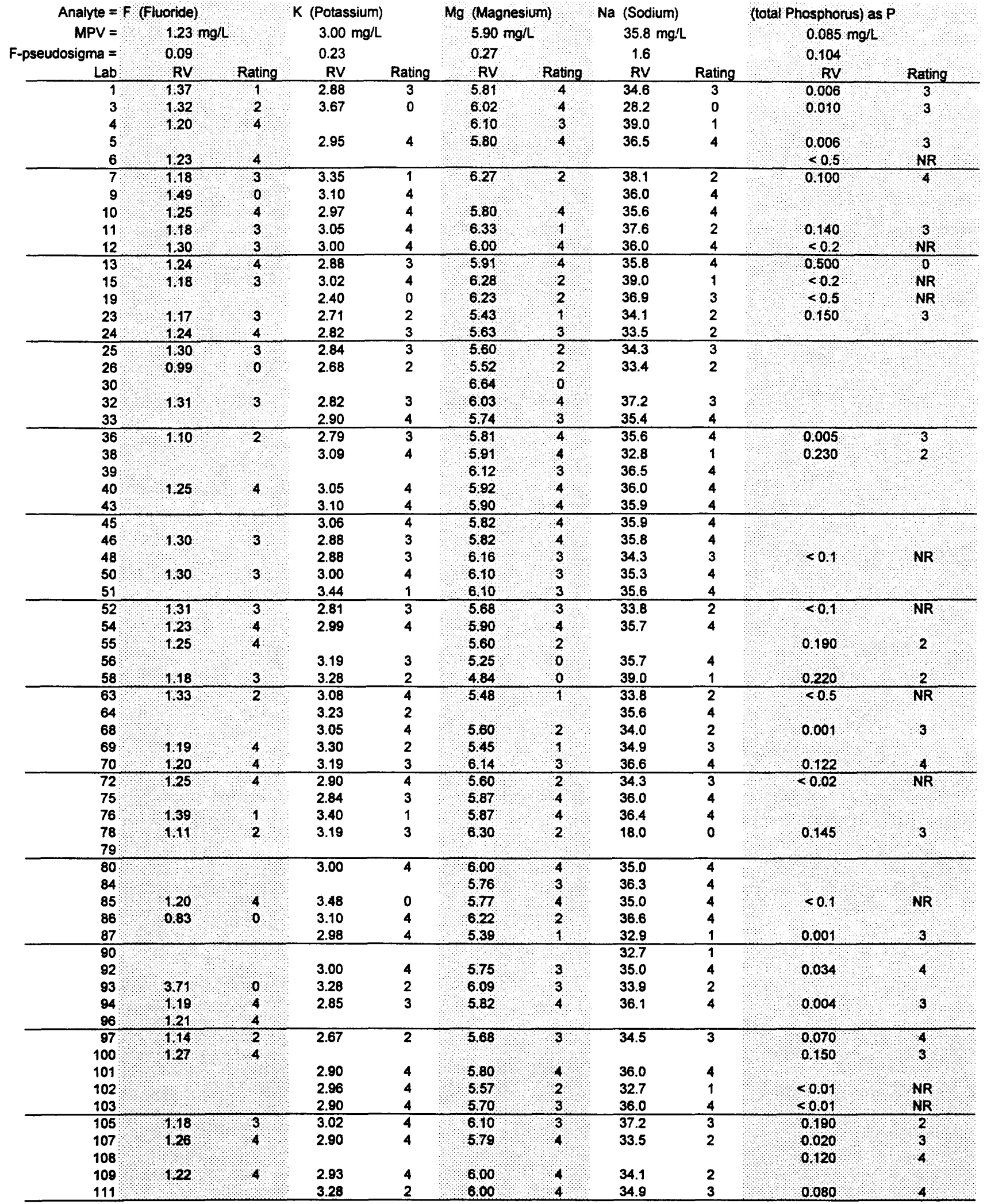


Table 6. -Laboratory performance ratings for standard reference water sample $M-130$ (major constituents) --Continued

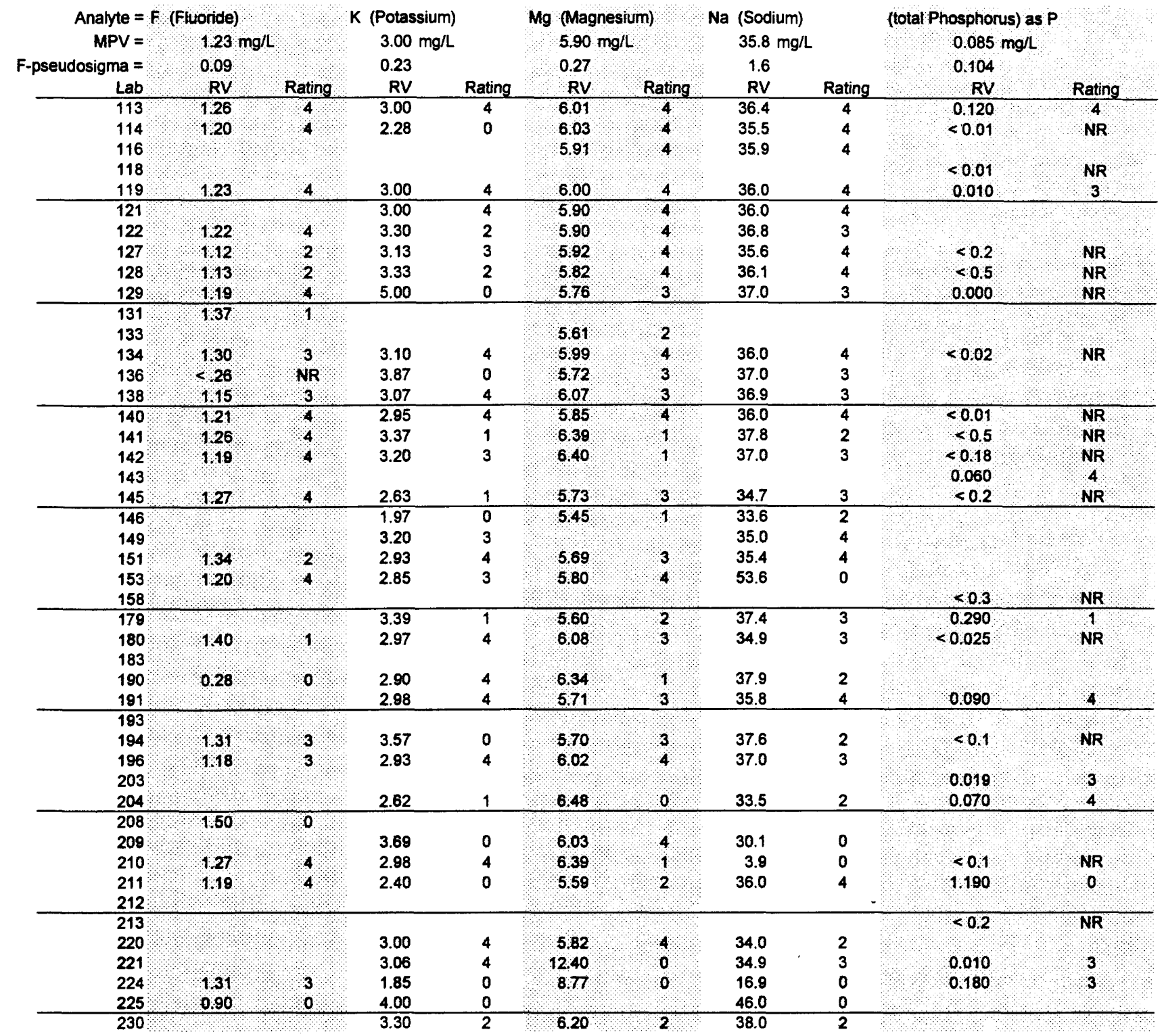


Table 6. -Laboratory performance ratings for standard reference water sample $M-130$ (major constituents)

-Continued

(MPV,most probable value; ug/L, micrograms per liter; mg/L, milligrams per liter; Lab, laboratory number; OLR, overall laboratory rating

for all reportad values; V/15, number of reported values of 16 possible values; RV, reported value; <, less than)

for all reportad values; V/15, number of reported values of 16 possible values; RV, reported value
\begin{tabular}{|llll|} 
Rating & Absolute Z-value & Rating & Absolute Z-value \\
4 (Excellent) & $0.00-0.50$ & 1 (Questionable) & $1.51-2.00$ \\
3 (Good) & $0.51-1.00$ & $O$ (Poor) & greatar than 2.00 \\
2 (Satisfactory) & $1.01-1.50$ & NR (Not Rated) & \\
\hline
\end{tabular}

\begin{tabular}{|c|c|c|c|c|c|c|c|c|c|c|c|c|}
\hline Anaiyte $=$ & & & $\mathrm{SiO} 2$ (Si & & SO4 KSu & & Sp Cond & & Sr (Stror & (um) & V Nanac & \\
\hline MPV $=$ & 802 & & 9.20 & & $\begin{array}{r}58.0 \\
\end{array}$ & & 335 & $\mu$ S.cm & $\begin{array}{r}180 \\
\end{array}$ & $\mu g / L$ & & $g / L$ \\
\hline F-pseudosigma $=$ & 0.14 & & 0.55 & & 2.6 & & 10 & & 10 & & insufficier & \\
\hline Lab & RV & Rating & RV & Rating & RV & Rating & $\mathrm{RV}$ & Rating & RV & Rating & RV & Rating \\
\hline 1 & 788 & 3 & 9.52 & 3 & 579 & 4 & 336 & 4 & 177. & 4 & 0.83 & NR \\
\hline 3 & 8.06 & 4 & 8.48 & 2 & 572 & 4 & 339 & 4 & 183 & 4 & $<5$ & NR \\
\hline 4 & & & 10.00 & 2 & 59.0 & 4 & & & 193 & 2 & $<10$ & NR \\
\hline 5 & 8.03 & 4 & 9.57 & 3 & 56.4 & 3 & 37 & 0 & 193 & 2 & $<4$ & NR \\
\hline 6 & $7 \vartheta 4$ & 3 & & & 58.0 & 4 & 350 & 2 & & & & \\
\hline 7 & 6.97 & 0 & & & 56.5 & 3 & 329 & 3 & 186 & 3 & $<4$ & NR \\
\hline 9 & & & 10.00 & 2 & 55.8 & 3 & 308 & 0 & & & & \\
\hline 10 & $8: 05$ & 4 & 9.20 & 4 & 59.0 & 4 & 337 & 4 & & & & \\
\hline 11 & 799 & 4 & 4.25 & 0 & 59.7 & 3 & 332 & 4 & 190 & 3 & & \\
\hline 12 & 8.00 & 4 & & & 60.0 & 3 & 342 & 3 & & & & \\
\hline 13 & 812 & 3 & 9.13 & 4 & 543 & 2 & 337 & 4 & & & & \\
\hline 15 & 739 & 0 & 8.86 & 3 & 510 & 0 & 346 & 2 & 171 & 3 & $<10$ & NR \\
\hline 19 & 8.02 & 4 & & & 56.3 & 3 & 314 & 1 & & & & \\
\hline 23 & 8,10 & 3 & 9.34 & 4 & 577 & 4 & 336 & 4 & & & 0.84 & NR \\
\hline 24 & 795 & 4 & 4.55 & 0 & 590 & 4 & 329 & 3 & 173 & 3 & & \\
\hline 25 & 787 & 2 & 8.90 & 3 & 68.4 & 0 & 343 & 3 & 175 & 4 & $<4$ & NR \\
\hline 26 & 730 & 0 & & & 558 & 3 & 354 & 1 & & & & \\
\hline 30 & 8.02 & 4 & & & 57.8 & 4 & & & & & & \\
\hline 32 & 8,13 & 3 & 8.70 & 3 & 57.6 & 4 & 308 & 0 & 177 & 4 & $<0.4$ & NR \\
\hline 33 & 790 & 3 & 9.63 & 3 & 56.0 & 3 & 318 & 1 & & & & \\
\hline 36 & 810 & 3 & 19.00 & 0 & 46.0 & 0 & 343 & 3 & & & & \\
\hline 38 & 8,10 & 3 & 9.31 & 4 & & & 343 & 3 & & & & \\
\hline 39 & & & 9.46 & 4 & & & & & 185 & 4 & $<2$ & NR \\
\hline 40 & 793 & 3 & 9.00 & 4 & 55.0 & 2 & 338 & 4 & 170 & 3 & & \\
\hline 43 & 780 & 1 & 9.80 & 2 & 570 & 4 & 338 & 4 & & & & \\
\hline 45 & 8.14 & 3 & 9.66 & 3 & 59.8 & 3 & 348 & 2 & & & & \\
\hline 46 & 789 & 3 & 9.37 & 4 & 58.3 & 4 & 332 & 4 & 183 & 4 & & \\
\hline 48 & 6.80 & 0 & 3.00 & 0 & 340.0 & 0 & & & & & $<4$ & NR \\
\hline 50 & 806 & 4 & 9.90 & 2 & 58.0 & 4 & 337 & 4 & & & & \\
\hline 51 & 782 & 2 & 9.58 & 3 & 61.7 & 2 & 327 & 3 & & & 0.20 & NR \\
\hline 52 & 8.10 & 3 & 4.82 & 0 & 57.6 & 4 & 316 & 1 & 160 & 1 & $<2$ & NR \\
\hline 54 & 8,03 & 4 & & & 610 & 2 & 328 & 3 & & & & \\
\hline 55 & 8,12 & 3 & 9.20 & 4 & 57.4 & 4 & 349 & 2 & 172 & 3 & & \\
\hline 56 & 793 & 3 & & & 59.8 & 3 & 328 & 3 & & & & \\
\hline 58 & 763 & 0 & 4.95 & 0 & 49,6 & 0 & 306 & 0 & & & & \\
\hline 63 & 8.04 & 4 & 8.88 & 3 & 43.9 & 0 & 280 & 0 & & & $<10$ & NR \\
\hline 64 & 8,05 & 4 & 9.00 & 4 & 620 & 1 & 346 & 2 & & & & \\
\hline 68 & 8.05 & 4 & 9.39 & 4 & & & 336 & 4 & 170 & 3 & $<3$ & NR \\
\hline 69 & 8.26 & 1 & & & 57.0 & 4 & 345 & 3 & & & & \\
\hline 70 & 784 & 2 & 9.11 & 4 & 58.7 & 4 & 322 & 2 & 187 & 3 & $<50$ & NR \\
\hline 72 & 8.04 & 4 & & & 51.3 & 0 & & & & & & \\
\hline 75 & 796 & 4 & & & 55.8 & 3 & 338 & 4 & & & & \\
\hline 76 & 703 & 3 & & & 58.5 & 4 & 334 & 4 & & & & \\
\hline 78 & 8.05 & 4 & 9.20 & 4 & 46.0 & 0 & 303 & 0 & & & 0.70 & NR \\
\hline 79 & 810 & 3 & & & & & 330 & 4 & & & & \\
\hline 80 & 780 & 1 & & & 610 & 2 & 333 & 4 & & & & \\
\hline 84 & 810 & 3 & & & & & 340 & 4 & & & & \\
\hline 85 & 812 & 3 & 9.20 & 4 & 55.5 & 3 & 331 & 4 & 180 & 4 & $<20$ & NR \\
\hline 86 & 8.10 & 3 & & & 65.4 & 0 & 326 & 3 & & & & \\
\hline 87 & 8.02 & 4 & 10.20 & 1 & 54.0 & 1 & 317 & 1 & & & & \\
\hline 90 & 789 & 4 & & & & & 320 & 2 & & & & \\
\hline 92 & 8.01 & 4 & 9.01 & 4 & 60.0 & 3 & 364 & 0 & & & & \\
\hline 93 & 804 & 4 & & & 60.7 & 2 & 302 & 0 & & & & \\
\hline 94 & 789 & 3 & & & 58.0 & 4 & 336 & 4 & 178 & 4 & $<5$ & NR \\
\hline 96 & 819 & 2 & & & 620 & 1 & 354 & 1 & & & & \\
\hline 97 & 815 & 3 & 8.83 & 3 & 30.3 & 0 & 340 & 4 & 143 & 0 & $<2.25$ & NR \\
\hline 100 & 821 & 2 & & & 574 & 4 & 331 & 4 & & & & \\
\hline 101 & 6.93 & 0 & 4.51 & 0 & & & 323 & 2 & & & & \\
\hline 102 & & & 9.00 & 4 & 58.0 & 4 & 361 & 0 & 182 & 4 & & \\
\hline 103 & & & 9.20 & 4 & & & & & 185 & 4 & $<5$ & NR \\
\hline 105 & 8.02 & 4 & 9.46 & 4 & 60.6 & 3 & 341 & 3 & 193 & 2 & $<20$ & NR \\
\hline 107 & 788 & 3 & 8.72 & 3 & & & 338 & 4 & & & & \\
\hline 108 & & & & & & & & & & & & \\
\hline 109 & 8.07 & 4 & 9.22 & 4 & 53.9 & 1 & 337 & 4 & & & & \\
\hline 111 & 8,30 & 1 & 2.05 & 0 & 85,2 & 0 & 328 & 3 & & & & \\
\hline
\end{tabular}


Table 6. -Laboratory performance ratings for standard reference water sample M-130 (major constituents)

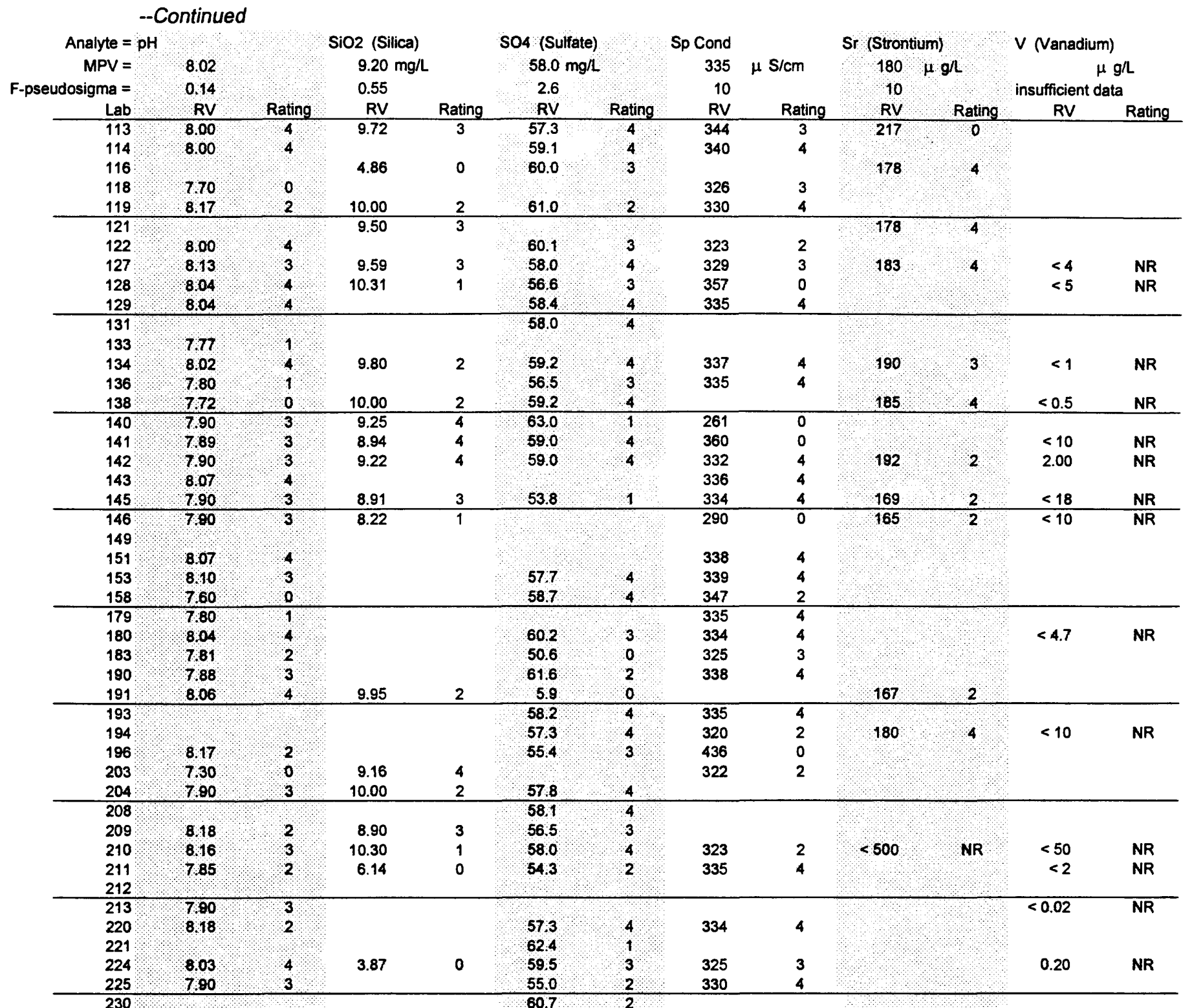


Table 7. -Laboratory performance ratings for standard reference water sample N-42 (preserved nutrients)

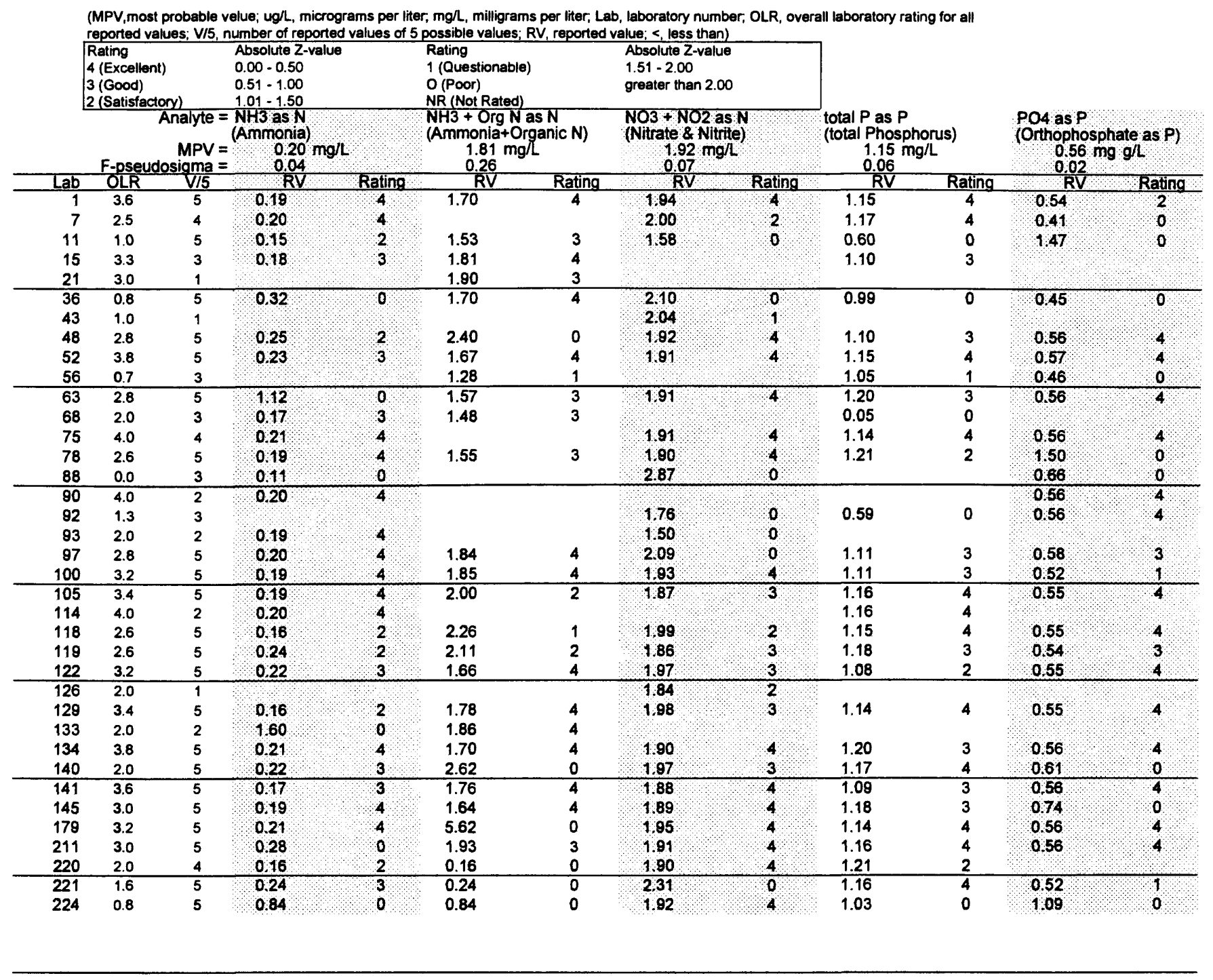


Table 7. -Laboratory performance ratings for standard reference water sample $\mathrm{N}-42$ (nonpreserved nutrients) -Continued

$\begin{array}{lllll}\text { Analyte }= & \mathrm{NH3} \text { as N } \\ \text { (Anmonia) }\end{array}$

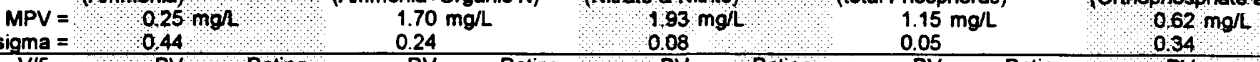

\begin{tabular}{|c|c|c|c|c|}
\hline & F-pseu & igma = & $\begin{array}{l}0.44 \\
\end{array}$ & \\
\hline Lab & OLR & V/5 & RV & Rating \\
\hline 3 & 3.0 & 5 & 0.41 & 4 \\
\hline 5 & 2.2 & 5 & 0.98 & 1 \\
\hline 6 & 1.5 & 4 & 0.74 & 2 \\
\hline 9 & 3.2 & 5 & 0.28 & 4 \\
\hline 10 & 3.6 & 5 & 023 & 4 \\
\hline 19 & 1.4 & 5 & 0.24 & 4 \\
\hline 12 & 3.3 & 4 & $<02$ & NR \\
\hline 13 & 4.0 & 4 & 0.41 & 4 \\
\hline 15 & 3.6 & 5 & 0,16 & 4 \\
\hline 19 & 2.8 & 4 & 0.25 & 4 \\
\hline 21 & 3.0 & 2 & & \\
\hline 22 & 4.0 & 1 & & \\
\hline 23 & 4.0 & 5 & 0.17 & 4 \\
\hline 25 & 2.5 & 4 & 034 & 4 \\
\hline 26 & 3.3 & 3 & 0.20 & 4 \\
\hline 32 & 4.0 & 3 & 0.23 & 4 \\
\hline 33 & 1.0 & 1 & 104 & 1 \\
\hline 36 & 2.4 & 5 & 0.36 & 4 \\
\hline 38 & 3.2 & 5 & 021 & 4 \\
\hline 45 & 2.8 & 4 & & \\
\hline 46 & 3.6 & 5 & 084 & 2 \\
\hline 51 & 2.8 & 5 & 0.21 & 4 \\
\hline 52 & 3.8 & 5 & 0,42 & 4 \\
\hline 53 & 2.0 & 2 & n & \\
\hline 54 & 1.0 & 1 & 0,99 & 1 \\
\hline 55 & 3.7 & 3 & & \\
\hline 56 & 0.0 & 1 & & \\
\hline 58 & 1.8 & 5 & 025 & 4 \\
\hline 59 & 3.4 & 5 & 0.19 & 4 \\
\hline 61 & 2.4 & 5 & 022 & 4 \\
\hline 63 & 2.4 & 5 & 0.22 & 4 \\
\hline 64 & 2.0 & 4 & 027 & 4 \\
\hline 68 & 3.5 & 2 & 0.40 & 4 \\
\hline 69 & 3.0 & 1 & & \\
\hline 70 & 2.2 & 5 & 0.20 & 4 \\
\hline 72 & 2.2 & 5 & 0.22 & 4 \\
\hline 75 & 2.3 & 4 & 0.96 & 1 \\
\hline 76 & 2.5 & 2 & 0.95 & 1 \\
\hline 78 & 2.0 & 5 & 0.23 & 4 \\
\hline 79 & 2.5 & 2 & & \\
\hline 80 & 0.7 & 3 & 1.13 & 1 \\
\hline 81 & 3.8 & 5 & 0.23 & 4 \\
\hline 84 & 3.0 & 3 & 0.30 & 4 \\
\hline 85 & 2.4 & 5 & 100 & 1 \\
\hline 87 & 3.8 & 5 & 0.42 & 4 \\
\hline 88 & 2.7 & 3 & 0,16 & 4 \\
\hline 90 & 3.7 & 3 & & \\
\hline 91 & 2.3 & 3 & 0,19 & 4 \\
\hline 92 & 2.0 & 4 & 0.20 & 4 \\
\hline 94 & 2.5 & 4 & 1.03 & 1 \\
\hline 98 & 2.6 & 5 & 0.98 & $\mathrm{~T}$ \\
\hline 97 & 3.4 & 5 & 0.21 & 4 \\
\hline 100 & 2.4 & 5 & 0.98 & 1 \\
\hline 102 & 1.8 & 5 & 1.50 & 0 \\
\hline 107 & 2.3 & 4 & 0,18 & 4 \\
\hline 108 & 1.5 & 4 & 0.46 & 4 \\
\hline 111 & 2.3 & 3 & 0.99 & 1 \\
\hline 113 & 3.3 & 4 & & \\
\hline 114 & 2.7 & 3 & 0.47 & 3 \\
\hline 118 & 4.0 & 5 & 0.18 & 4 \\
\hline 119 & 3.2 & 5 & 0.25 & 4 \\
\hline 122 & 3.6 & 5 & $0.1 \mathrm{~B}$ & 4 \\
\hline 127 & 3.8 & 5 & 0.35 & 4 \\
\hline 128 & 2.0 & 5 & 1.04 & 1 \\
\hline 129 & 3.8 & 5 & 0.23 & 4 \\
\hline 133 & 3.0 & 3 & & \\
\hline 134 & 3.4 & 5 & 021 & 4 \\
\hline 136 & 3.0 & 3 & 0.18 & 4 \\
\hline 138 & 3.6 & 5 & 020 & 4 \\
\hline 142 & 2.0 & 5 & 0.24 & 4 \\
\hline 143 & 3.6 & 5 & 0.19 & 4 \\
\hline 145 & 3.0 & 5 & 094 & 1 \\
\hline 146 & 1.0 & 2 & & \\
\hline 158 & 2.8 & 4 & 0.98 & 1 \\
\hline 179 & 2.8 & 5 & 0.68 & 3 \\
\hline 180 & 3.8 & 5 & 020 & 4 \\
\hline 183 & 1.0 & 2 & & \\
\hline 190 & 2.7 & 3 & 025 & 4 \\
\hline $\begin{array}{l}191 \\
193\end{array}$ & $\begin{array}{l}3.0 \\
4.0\end{array}$ & 2 & & \\
\hline$\frac{193}{194}$ & $\frac{4.0}{1.8}$ & $\frac{1}{4}$ & 0.18 & 4 \\
\hline $\begin{array}{l}194 \\
196\end{array}$ & 3.0 & & & \\
\hline 197 & 4.0 & 2 & 020 & 4 \\
\hline 203 & 1.3 & 4 & 0.22 & 4 \\
\hline 204 & 3.3 & 4 & 0.19 & 4 \\
\hline & 0.0 & 1 & & \\
\hline 208 & 3.5 & 2 & & \\
\hline 210 & 3.2 & 5 & 020 & 4 \\
\hline 211 & 2.6 & 5 & 0.97 & 1 \\
\hline 213 & 3.0 & 4 & 0.25 & 4 \\
\hline & 2.3 & & 0.20 & \\
\hline 224 & 2.8 & 5 & 019 & 4 \\
\hline $\begin{array}{l}225 \\
227\end{array}$ & $\begin{array}{l}0.8 \\
3.5\end{array}$ & $\begin{array}{l}5 \\
2\end{array}$ & 134 & 0 \\
\hline
\end{tabular}


Table 8. - Laboratory performance ratings for standard reference water sample P-22 (low ionic strength)

(MPV,most probable value; ug/L, micrograms per liter; mg/L, milligrams per liter, Lab, laboratory number; OLR, overall laboratory rating for

for all reported values; V/11, number of reported values of 11 possible values; RV, reported value; <, lass than)

\begin{tabular}{|llll|}
\hline Rating & Absolute Z-value & Rating & Absolute Z-value \\
4 (Excellent) & $0.00-0.50$ & 1 (Questionable) & $1.51-2.00$ \\
3 (Good) & $0.51-1.00$ & $O$ (Poor) & greater than 2.00 \\
2 (Satisfactory) & $1.01-1.50$ & NR (Not Rated) & \\
\hline
\end{tabular}

2 (Satisfactory) $\quad 1.01-1.50$

NR (Not Rated)

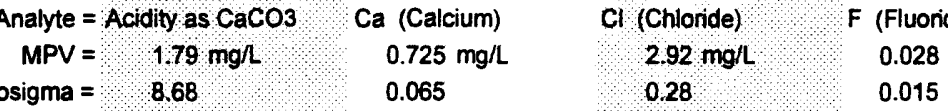

$0.725 \mathrm{mg} / \mathrm{L}$

Cl (Chloride)

0.065

$2.92 \mathrm{mg} / \mathrm{L}$

0.015

F (Fluoride)

F-pseudosigma $=$

K (Potassium) $0.203 \mathrm{mgh}$ 0.027
Mg (Magnesium) $0.098 \mathrm{mg} / \mathrm{L}$ 0.006

\begin{tabular}{|c|c|c|c|c|c|c|c|c|c|c|c|c|c|c|}
\hline Lab & OLR & $\mathrm{V} / 11$ & $\mathrm{RV}$ & Rating & RV & Rating & RV & Rating & RV & Rating & $\mathrm{RV}$ & Rating & RV & Rating \\
\hline 1 & 3.0 & 10 & 112 & 4 . & 0.730 & 4 & 2.96 & 4 & 0.051 & 1 & 0.232 & 2 & 0.099 & 4 \\
\hline 2 & 1.9 & 7 & & & 1.148 & 0 & & & & & 0.207 & 4 & 0.117 & 0 \\
\hline 3 & 1.6 & 7 & $\begin{array}{l}<10 \\
\end{array}$ & NR & 0.580 & 0 & 216 & 0 & $<0.1$ & NR & 0,150 & 1 & 0.094 & 3 \\
\hline 5 & 1.7 & 7 & & & 0.719 & 4 & 080 & 0 & & & $<1$ & NR & 0.100 & 4 \\
\hline 7 & 3.0 & 4 & & & & & 2.50 & 2 & $<0.5$ & NR & & & & \\
\hline 11 & 2.9 & 9 & 126 & 4 & 0.770 & 3 & 2.62 & 2 & & & 0,230 & 3 & 0.100 & 4 \\
\hline 15 & 2.5 & 10 & 176 & 4 & 0.802 & 2 & 3.47 & 1 & 0.029 & 4 & 0.202 & 4 & 0.098 & 4 \\
\hline 23 & 3.1 & 11 & 157 & 4 & 0.767 & 3 & 3.02 & 4 & 0.021 & 4 & 0,104 & 4 & 0.096 & 4 \\
\hline 25 & 2.8 & 10 & 260 & 4 & 0.636 & 2 & 2.92 & 4 & 0.026 & 4 & - 121 & $\mathbf{N R}$ & 0.088 & 1 \\
\hline 26 & 1.0 & 5 & & & 0.960 & 0 & 2.87 & 4 & $<0.1$ & NR & 0,260 & 0 & 0.110 & 1 \\
\hline 33 & 3.4 & 8 & & & 0.682 & 3 & 287 & 4 & & & 0.217 & 3 & 0.092 & 2 \\
\hline 36 & 2.2 & 9 & 110 & 4 & 0.672 & 3 & 470 & 0 & 0.020 & 3 & 0.202 & 4 & 0.160 & 0 \\
\hline 38 & 4.0 & 8 & 179 & 4 & 0.730 & 4 & & & & & 0210 & 4 & 0.101 & 4 \\
\hline 39 & 3.3 & 3 & & & 0.688 & 3 & & & & & & & 0.099 & 4 \\
\hline 44 & 2.8 & 6 & & & 0.735 & 4 & 3.43 & 1 & & & 0.215 & 4 & 0.097 & 4 \\
\hline 46 & 3.4 & 8 & & & 0.723 & 4 & 3.51 & 0 & & & 01194 & 4 & 0.096 & 4 \\
\hline 48 & 1.8 & 8 & & & 0.820 & 2 & 3.00 & 4 & & & 0.220 & 3 & 0.060 & 0 \\
\hline 52 & 3.0 & 7 & & & 0.721 & 4 & 311 & 3 & $<0.05$ & NR & $<0.5$ & NR & 0.112 & 0 \\
\hline 58 & 1.5 & 11 & 10,60 & 2 & 0.500 & 0 & 2.33 & 0 & 0.007 & 2 & 0,110 & 0 & 0.093 & 3 \\
\hline 61 & 2.6 & 10 & 0.40 & 4 & 0.626 & 1 & 3.00 & 4 & 0.030 & 4 & 0.100 & 0 & 0.093 & 3 \\
\hline 62 & 3.5 & 2 & & & & & & & & & & & & \\
\hline 63 & 2.6 & 9 & 100 & 4 & 0.943 & 0 & 300 & 4 & $<0.2$ & NR & 0.230 & 3 & 0.163 & 0 \\
\hline 64 & 3.6 & 9 & & & 0.670 & 3 & 2.97 & 4 & & & 0.200 & 4 & 0.100 & 4 \\
\hline 78 & 2.8 & 10 & 200 & 4 & 0.750 & 4 & 2.50 & 2 & 0.041 & 3 & 0,180 & 3 & 0.100 & 4 \\
\hline 92 & 3.0 & 2 & & & & & 3.00 & 4 & & & & & & \\
\hline 93 & 2.6 & 9 & & & 0.638 & 2 & 326 & 2 & 0.016 & 3 & 0.215 & 4 & 0.060 & 0 \\
\hline 94 & 3.0 & 8 & 24.50 & 0 & 0.725 & 4 & 280 & 4 & $<0.1$ & NR & < 1 & NR & 0.103 & 3 \\
\hline 100 & 2.3 & 6 & & & & & 418 & 0 & 0.032 & 4 & & & & \\
\hline 101 & 2.6 & 7 & & & 0.747 & 4 & 130 & 0 & & & 02210 & 4 & 0.105 & 2 \\
\hline 102 & 1.6 & 5 & & & 0.710 & 4 & $<10$ & NR & & & 0,007 & 0 & 0.087 & 1 \\
\hline 107 & 3.9 & 9 & & & 0.720 & 4 & 3.00 & 4 & 0.016 & 3 & 0,190 & 4 & 0.100 & 4 \\
\hline 110 & 3.7 & 9 & & & 0.760 & 3 & 290 & 4 & 0.020 & 4 & 0,210 & 4 & 0.100 & 4 \\
\hline 111 & 3.0 & 8 & & & 0.710 & 4 & 2.95 & 4 & & & 0.220 & 3 & 0.090 & 2 \\
\hline 112 & 3.4 & 7 & & & 0.672 & 3 & 282 & 4 & & & 0.194 & 4 & 0.091 & 2 \\
\hline 113 & 2.8 & 6 & & & $<1$ & NR & 3.19 & 3 & 0.024 & 4 & 0.280 & 0 & $<0.2$ & NR \\
\hline 134 & 2.9 & 8 & & & 0.760 & 3 & 316 & 3 & $<0.1$ & NR & 0.203 & 4 & 0.090 & 2 \\
\hline 136 & 1.8 & 9 & 15.20 & 1 & 0.840 & 1 & 353 & 0 & $<0.26$ & NR & 0,150 & 1 & 0.168 & 0 \\
\hline 138 & 3.1 & 7 & & & 0.740 & 4 & 270 & 3 & & & 0.210 & 4 & 0.099 & 4 \\
\hline 140 & 2.8 & 8 & & & 0.725 & 4 & 292 & 4 & 0.012 & 2 & 0.220 & 3 & 0.092 & 2 \\
\hline 141 & 2.1 & 8 & 2230 & 0 & 0.614 & 1 & 258 & 2 & $<0.1$ & NR & 0.211 & 4 & 0.094 & 3 \\
\hline 143 & 3.0 & 4 & & & & & 2.45 & 1 & & & & & & \\
\hline 145 & 3.3 & 7 & 200 & 4 & 0.700 & 4 & 2.87 & 4 & $<0.2$ & NR & $<0707$ & NR & $<0.19$ & NR \\
\hline 158 & 2.4 & 8 & & & 0.950 & 0 & 3.07 & 3 & & & $0.0 B 0$ & 0 & & \\
\hline 180 & 2.7 & 7 & 150 & 4 & 0.642 & 2 & 2.72 & 3 & $<0.1$ & NR & $<12$ & NR & 0.093 & 3 \\
\hline 190 & 1.9 & 9 & & & 1.120 & 0 & 2.82 & 4 & 0.035 & 3 & 0.176 & 3 & 0.119 & 0 \\
\hline 194 & 3.0 & 3 & & & $<5$ & NR & 2.50 & 2 & $<0.2$ & NR & $<0.5$ & NR & $<1$ & NR \\
\hline 196 & 2.8 & 9 & 105 & 4 & 0.758 & 3 & 2.81 & 4 & $<0.5$ & NR & 0.190 & 4 & 0.107 & 2 \\
\hline 203 & 2.3 & 4 & & & & & 4,10 & 0 & & & & & & \\
\hline 204 & 2.6 & 8 & & & 0.565 & 0 & 278 & 4 & & & 0,162 & 2 & 0.100 & 4 \\
\hline 209 & 3.6 & 7 & & & 0.694 & 4 & 281 & 4 & & & 0.171 & 2 & 0.096 & 4 \\
\hline 210 & 2.0 & 7 & $\begin{array}{l}<2 \\
\end{array}$ & NR & 0.545 & 0 & 2.58 & 2 & 0.040 & 3 & $<1$ & $\mathrm{NR}$ & 0.095 & 3 \\
\hline 224 & 2.6 & 11 & 2730 & 0 & 0.728 & 4 & 300 & 4 & 0.060 & 0 & 0,185 & 3 & 0.088 & 1 \\
\hline 225 & 0.8 & 9 & 20,00 & 0 & 0.800 & 2 & 700 & 0 & 0.130 & 0 & 0.500 & 0 & & \\
\hline
\end{tabular}


Table 8. -Laboratory performance ratings for standard reference water sample P-22 (low ionic strength)

-Continued

(MPV,most probable value; ug/L, micrograms per liter; mg/L, milligrams per liter; Lab, laboratory number; OLR, overall laboratory rating for all reported values; V/11, number of reported values of 11 possible values; RV, reported value; <, less than)

\begin{tabular}{|llll} 
Rating & Absolute Z-value & Rating & Absolute Z-value \\
4 (Excellent) & $0.00-0.50$ & 1 (Questionable) & $1.51-2.00$ \\
3 (Good) & $0.51-1.00$ & $O$ (Poor) & greater than 2.00 \\
2 (Satisfactory) & $1.01-1.50$ & NR (Not Rated) & \\
\hline
\end{tabular}

Analyte $=\mathrm{Na}(\mathrm{Sodium}), \mathrm{pH}$

MPV =

$170 \mathrm{mg} / \mathrm{l}$

5.8

F-pseudosigma $=$

010

0.19

PO4 as

$0.008 \mathrm{mg} / \mathrm{L}$

SO4 (Sulfate)

0.006

$0.728 \mathrm{mg}$ 'L

Specific Conductance

$17.0 \mu \mathrm{S} / \mathrm{cm}$

\begin{tabular}{|c|c|c|c|c|c|c|c|c|c|c|}
\hline Lab & RV & Rating & RV & Rating & RV & Rating & RV & Rating & RV & Rating \\
\hline 1 & 178 & 3 & 7.27 & 0 & $<0.001$ & NR & 0.688 & 4 & 17.5 & 4 \\
\hline 2 & 1,88 & 1 & 5.89 & 4 & & & 0.728 & 4 & 13.5 & 0 \\
\hline 3 & 110 & 0 & 5.93 & 3 & $<0.01$ & NR & $<1$ & NR & 16.7 & 4 \\
\hline 5 & 182 & 2 & 6.07 & 2 & $<0.005$ & NR & 2.890 & 0 & 18.8 & 0 \\
\hline 7 & & & 5.94 & 3 & $<0.01$ & NR & 0.650 & 4 & 16.1 & 3 \\
\hline 11 & 173 & 4 & 7.85 & 0 & & & 1.030 & 3 & 16.3 & 3 \\
\hline 15 & 1.84 & 2 & 4.85 & 0 & $<0.02$ & NR & 1.220 & 2 & 18.8 & 2 \\
\hline 23 & 1.67 & 4 & 5.86 & 4 & 0,900 & 0 & 1.580 & 0 & 16.3 & 3 \\
\hline 25 & 1.67 & 4 & 5.77 & 4 & 0.008 & 4 & 2.590 & 0 & 19.0 & 1 \\
\hline 26 & 107 & 0 & & & $<0,7$ & NR & $<0.5$ & NR & & \\
\hline 33 & 176 & 3 & 5.73 & 4 & $<0.01$ & NR & 0.670 & 4 & 16.5 & 4 \\
\hline 36 & 1.59 & 2 & 5.90 & 4 & 0.000 & NR & 0.000 & NR & 19.8 & 0 \\
\hline 38 & 168 & 4 & 5.90 & 4 & 0.009 & 4 & & & 17.2 & 4 \\
\hline 39 & 165 & 3 & & & & & & & & \\
\hline 44 & 1.49 & 0 & & & & & 0.677 & 4 & & \\
\hline 46 & 172 & 4 & 5.73 & 4 & & & 0.410 & 3 & 17.1 & 4 \\
\hline 48 & 202 & 0 & 7.00 & 0 & $<0.005$ & NR & 0.057 & 1 & 16.5 & 4 \\
\hline 52 & 1.67 & 4 & 5.94 & 3 & $<0.005$ & NR & 0.950 & 3 & 16.4 & 4 \\
\hline 58 & 110 & 0 & 4.17 & 0 & 0.004 & 3 & 1.240 & 2 & 16.7 & 4 \\
\hline 61 & 1.62 & 3 & 5.68 & 3 & $<0.01$ & NR & 3.000 & 0 & 17.6 & 4 \\
\hline 62 & & & 5.84 & 4 & & & & & 16.0 & 3 \\
\hline 63 & 165 & 3 & 5.88 & 4 & $<0.05$ & NR & 0.456 & 3 & 15.7 & 2 \\
\hline 64 & 1.65 & 3 & 5.86 & 4 & 0.002 & 2 & 0.700 & 4 & 17.0 & 4 \\
\hline 78 & 180 & 2 & 6.03 & 2 & 0.008 & 4 & $<1$ & NR & 28.0 & 0 \\
\hline 92 & & & 6.04 & 2 & & & $<5$ & NR & & \\
\hline 93 & 168 & 4 & 5.69 & 3 & & & 1.180 & 2 & 15.8 & 3 \\
\hline 94 & 1.78 & 3 & 5.70 & 3 & & & 1.000 & 3 & 16.8 & 4 \\
\hline 100 & & & 5.96 & 3 & 0.010 & 4 & 3.000 & 0 & 18.1 & 3 \\
\hline 101 & 170 & 4 & 4.64 & 0 & & & & & 17.0 & 4 \\
\hline 102 & 1,61 & 3 & & & $<0,005$ & NR & $<10$ & NR & 13.0 & 0 \\
\hline 107 & 170 & 4 & 5.81 & 4 & 0.010 & 4 & & & 16.9 & 4 \\
\hline 110 & 170 & 4 & 5.60 & 2 & & & 0.785 & 4 & 17.0 & 4 \\
\hline 111 & 1.71 & 4 & 6.48 & 0 & $<0.001$ & NR & 0.672 & 4 & 16.1 & 3 \\
\hline 112 & 161 & 3 & 5.77 & 4 & & & 0.690 & 4 & & \\
\hline 113 & 1.80 & 2 & 5.81 & 4 & $<0.004$ & NR & $<2$ & NR & 17.4 & 4 \\
\hline 134 & 180 & 2 & 5.50 & 1 & $<0.01$ & NR & 0.920 & 4 & 176 & 4 \\
\hline 136 & 1.70 & 4 & 5.60 & 2 & & & 0.750 & 4 & 18.0 & 3 \\
\hline 138 & 177 & 3 & 5.27 & 0 & $<0.02$ & NR & 0.680 & 4 & & \\
\hline 140 & 172 & 4 & 5.66 & 3 & $<0.01$ & NR & $<2$ & NR & 13.1 & 0 \\
\hline 141 & 176 & 3 & 5.76 & 4 & $<0.05$ & NR & $<10$ & NR & 33.5 & 0 \\
\hline 143 & & & 5.72 & 4 & 0.003 & 3 & & & 16.5 & 4 \\
\hline 145 & 1,65 & 3 & 5.50 & 1 & $<0.01$ & NR & 0.600 & 4 & 18.0 & 3 \\
\hline 158 & 1.66 & 4 & 5.72 & 4 & 0.110 & 0 & 0.600 & 4 & 17.4 & 4 \\
\hline 180 & 162 & 3 & 5.81 & 4 & $<0.025$ & NR & $<2.5$ & NR & 20.0 & 0 \\
\hline 190 & 183 & 2 & 5.60 & 2 & 0.000 & NR & 1.990 & 0 & 18.0 & 3 \\
\hline 194 & $<5$ & NR & 5.86 & 4 & & & $<10$ & NR & 160 & 3 \\
\hline 196 & 177 & 3 & 5.44 & 1 & $<0.1$ & NR & 0.700 & 4 & 20.8 & 0 \\
\hline 203 & & & 5.60 & 2 & 0.010 & 4 & & & 16.1 & 3 \\
\hline 204 & 1.53 & 1 & 5.75 & 4 & 0.002 & 2 & 0.910 & 4 & & \\
\hline 209 & 1.63 & 3 & 5.74 & 4 & & & 0.710 & 4 & & \\
\hline 210 & 198 & 0 & 5.99 & 3 & $<0.05$ & NR & $<5$ & NR & 16.2 & 3 \\
\hline 224 & 1669 & 4 & 5.95 & 3 & 0.005 & 3 & 0.680 & 4 & 18.0 & 3 \\
\hline 225 & 3.50 & 0 & 6.20 & 1 & & & 2.000 & 0 & 17.0 & 4 \\
\hline
\end{tabular}


Table 9. -Laboratory performance ratings for standard reference water sample $\mathrm{Hg}-18$ (mercury)

(MPV, most probable value; ugl, micrograms per liter, Lab, laboratory number VII number of reported values of 1 value; RV, reported value; <, less than)

\begin{tabular}{|llll|}
\hline Rating & Absoluta Z-value & Rating & Absolute Z-valua \\
4 (Excellent) & $0.00-0.50$ & 1 (Questionable) & $1.51-2.00$ \\
3 (Good) & $0.51-1.00$ & $O$ (Poor) & greater than 2.00 \\
2 (Satisfactory) & $1.01-1.50$ & NR (Not Ratad) & \\
\hline
\end{tabular}

2 (Satisfactory) $1.01-150$ NR (Not Ratad)

Analyte $=\mathrm{Hg}$ (Mercuny)

MPV $=0.70 \mu \mathrm{g} / \mathrm{L}$

F-pseudosigma $=0.12$

$\begin{array}{ccc}\text { Lab } & \text { V } / 1 \\ 1 & \mathrm{RV}, \mathrm{Rating}\end{array}$

\begin{tabular}{|c|c|c|}
\hline 1 & 077 & 3 \\
\hline 3 & 078 & 3 \\
\hline 7 & 0,53 & 2 \\
\hline 11 & 0.66 & 4 \\
\hline 12 & 080 & 3 \\
\hline 13 & 1.07 & 0 \\
\hline 15 & 0,67 & 4 \\
\hline 24 & 0,70 & 4 \\
\hline 32 & 075 & 4 \\
\hline 34 & 0.66 & 4 \\
\hline 36 & 0.64 & 3 \\
\hline 39 & 100 & 0 \\
\hline 45 & 0.86 & 2 \\
\hline 46 & 0.71 & 4 \\
\hline 48 & 0.65 & 4 \\
\hline 50 & 090 & 1 \\
\hline 51 & 0,53 & 2 \\
\hline 52 & 0.51 & 1 \\
\hline 55 & 0,69 & 4 \\
\hline 58 & 0.80 & 3 \\
\hline 59 & 0.70 & 4 \\
\hline 61 & 0.76 & 3 \\
\hline 63 & 0.80 & 3 \\
\hline 68 & 0.52 & 1 \\
\hline 69 & 0.65 & 4 \\
\hline 70 & 0.60 & 3 \\
\hline 75 & 0.65 & 4 \\
\hline 76 & 0.62 & 3 \\
\hline 78 & 071 & 4 \\
\hline 79 & 0.76 & 3 \\
\hline 81 & 0.60 & 3 \\
\hline 86 & 0.66 & 4 \\
\hline 87 & 0.60 & 3 \\
\hline 90 & 0.63 & 3 \\
\hline 92 & 3.00 & 0 \\
\hline 96 & 0.70 & 4 \\
\hline 97 & 0.68 & 4 \\
\hline 100 & 106 & 0 \\
\hline 105 & 105 & 0 \\
\hline 108 & 0.66 & 4 \\
\hline 109 & 0.67 & 4 \\
\hline 113 & 120 & o \\
\hline 114 & 127 & 0 \\
\hline 118 & 170 & 0 \\
\hline 119 & 070 & 4 \\
\hline 127 & 079 & 3 \\
\hline 128 & 100 & 0 \\
\hline 133 & 0.55 & 2 \\
\hline 134 & 072 & 4 \\
\hline 138 & 0.68 & 4 \\
\hline 141 & 070 & 4 \\
\hline 142 & 0.84 & 2 \\
\hline 145 & 0.53 & 2 \\
\hline 146 & 0.66 & 4 \\
\hline 149 & 065 & 4 \\
\hline 151 & 0.67 & 4 \\
\hline 180 & 0,60 & 3 \\
\hline 194 & 0,80 & 3 \\
\hline 198 & 0883 & 2 \\
\hline 204 & 0.80 & 3 \\
\hline 210 & 081 & 3 \\
\hline 211 & $<0,3$ & 0 \\
\hline 220 & 0,57 & 2 \\
\hline 221 & 0,90 & 1 \\
\hline 225 & 200 & 0 \\
\hline
\end{tabular}


Definition of analytical methods, abbreviations, and symbols.

Analytical methods

0. Other/Not reported

1. AA: direct air

atomic absorption: direct, air

2. AA: direct $\mathrm{N} 2 \mathrm{O}$

atomic absorption: direct, nitrous oxide

3. AA: graphite furnace

atomic absorption: graphite fumace

4. ICP

5. DCP

6. ICPMS

11. AA: hydride

inductively coupled plasma

12. Flame emission

22: Color

direct current plasma

inductively coupled plasma/mass spectrometry

atomic absorption: hydride [reducing agent specified]

colorimetric [color reagent specified]

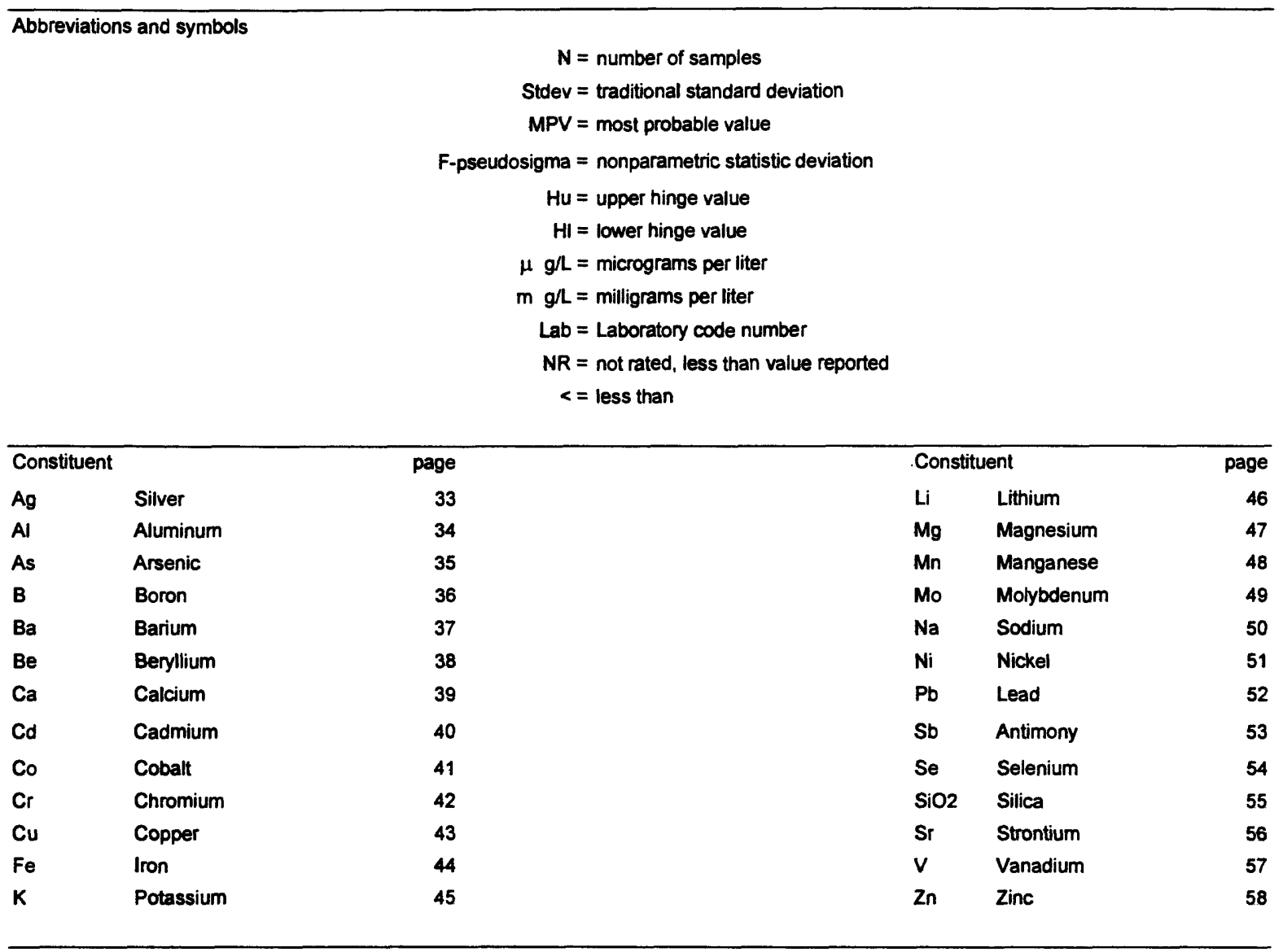


Table 10. -Statistical summary of reported data for standard reference water sample $T-129$ (trace constituents)--Continued Ag (Silver) $\mu \mathrm{g} / \mathrm{L}$
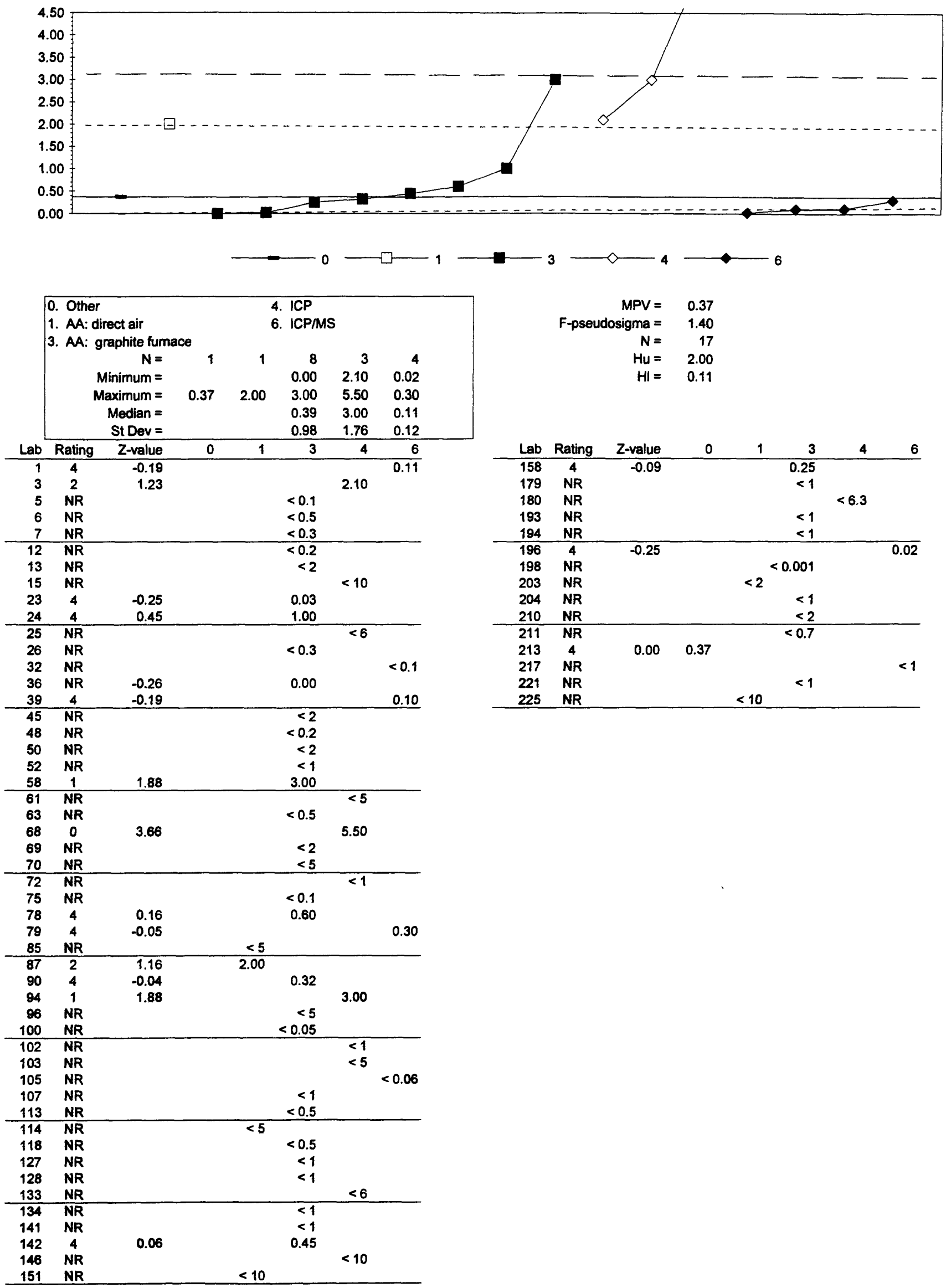
Table 10. -Statistical summary of reported data for standard reference water sample T-129 (trace constituents)--Continued Al (Aluminum) $\mu \mathrm{g} / \mathrm{L}$

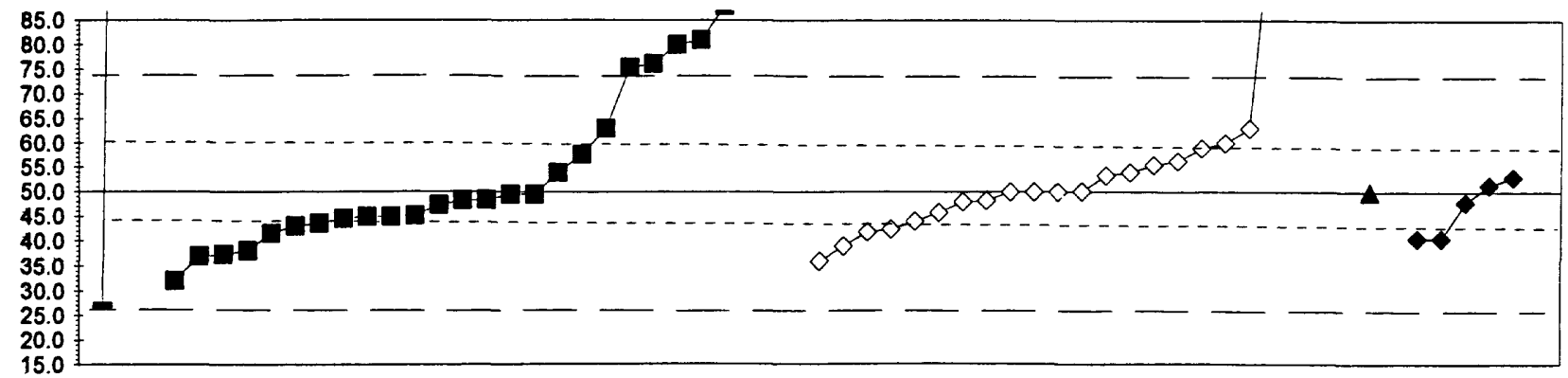

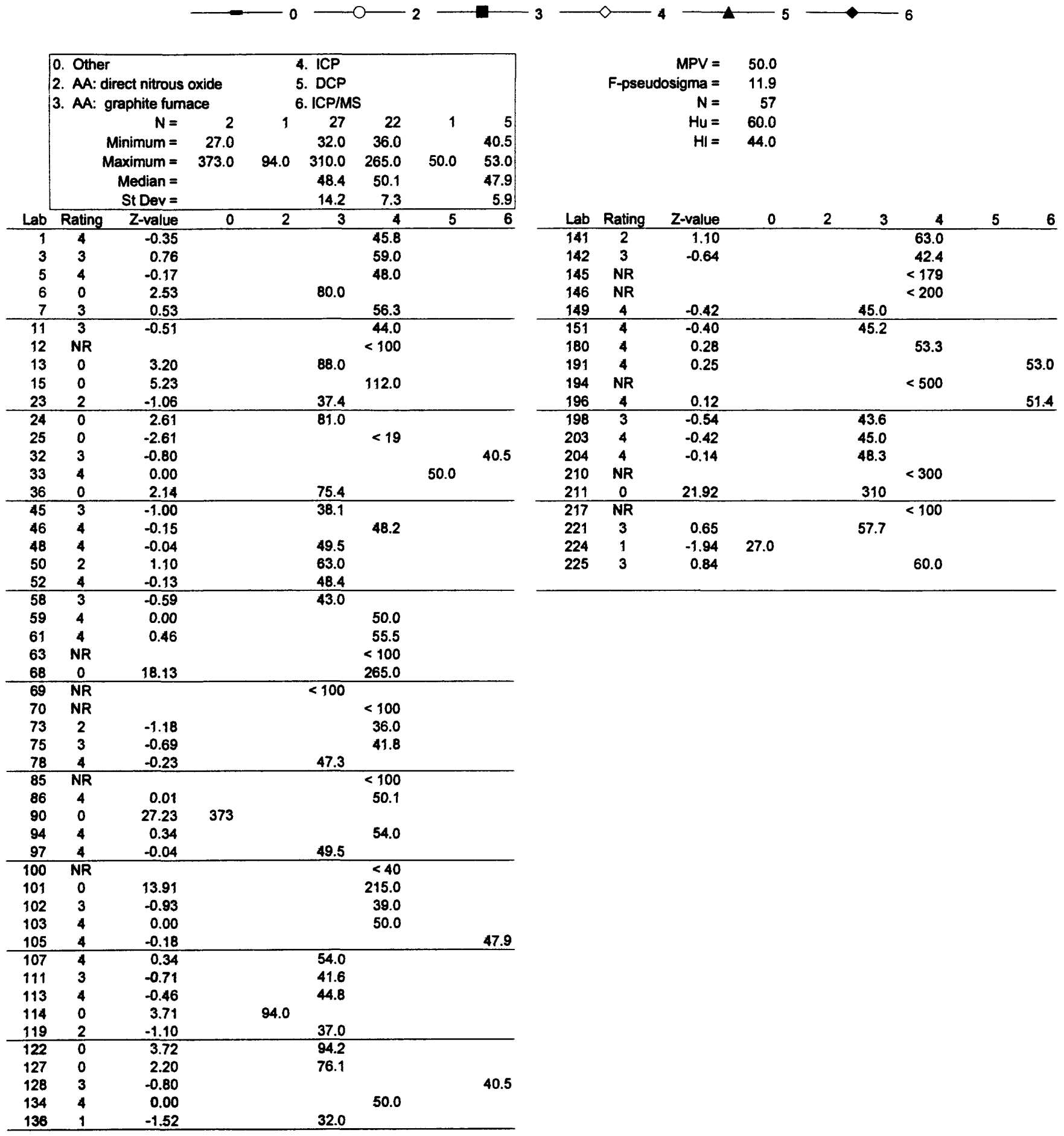


Table 10. -Statistical summary of reported data for standard reference water sample T-129 (trace constituents)--Continued As (Arsenic)

$\mu \mathrm{g} / \mathrm{L}$
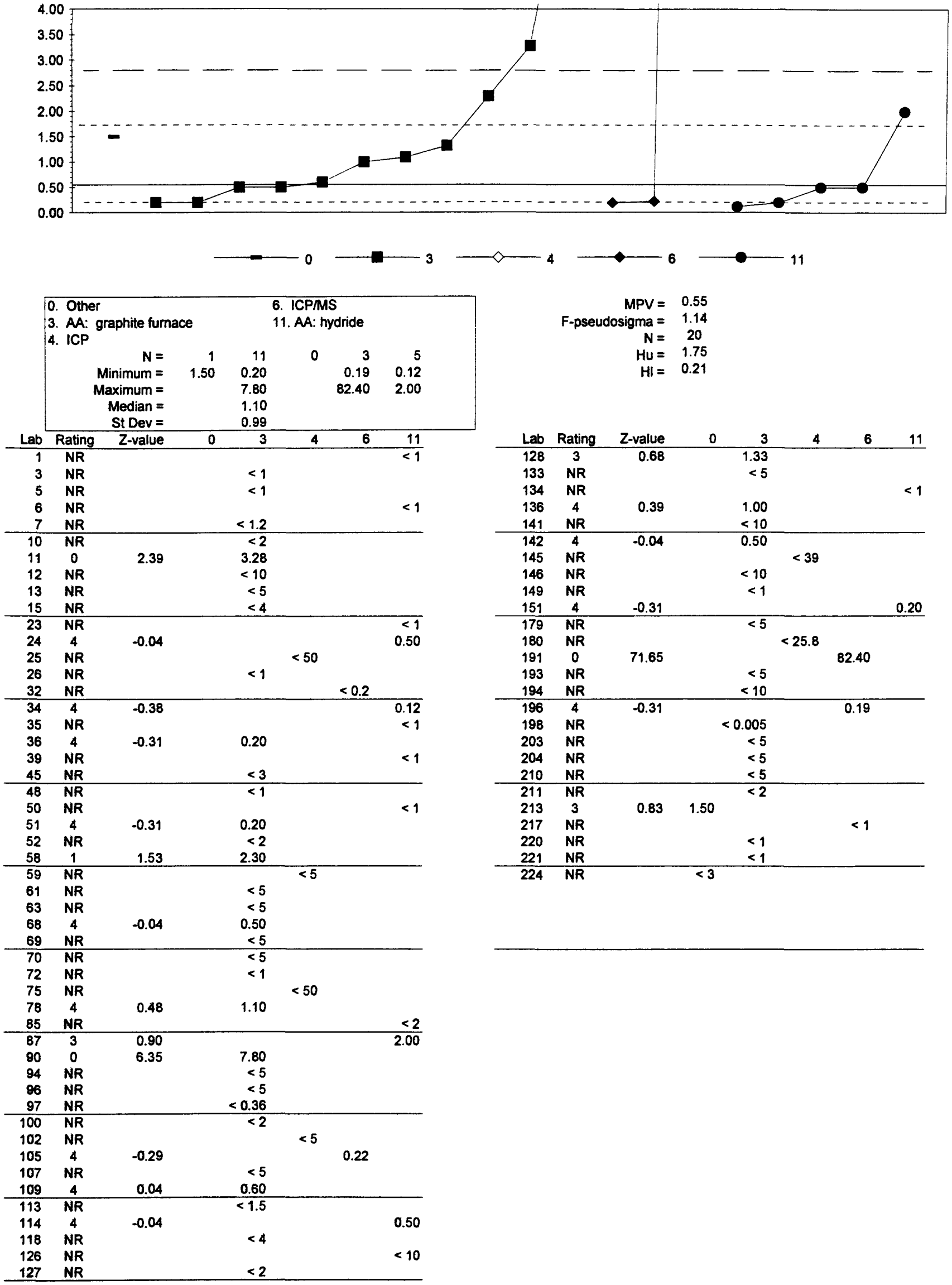
Table 10. -Statistical summary of reported data for standard reference water sample $T-129$ (trace constituents)--Continued B (Boron) $\mu \mathrm{g} / \mathrm{L}$
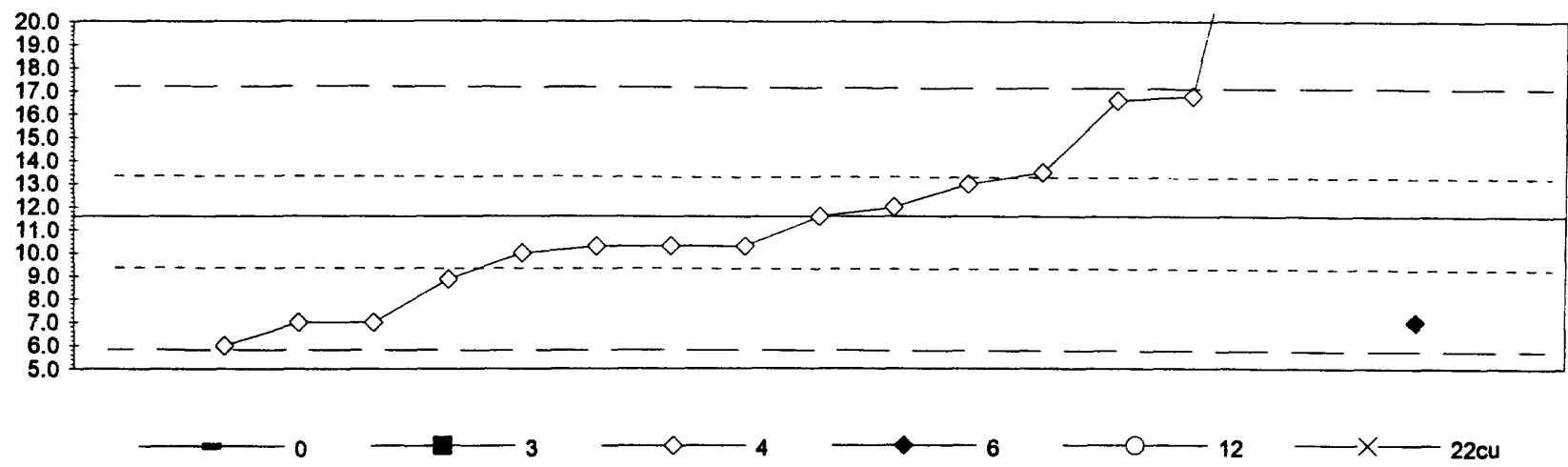

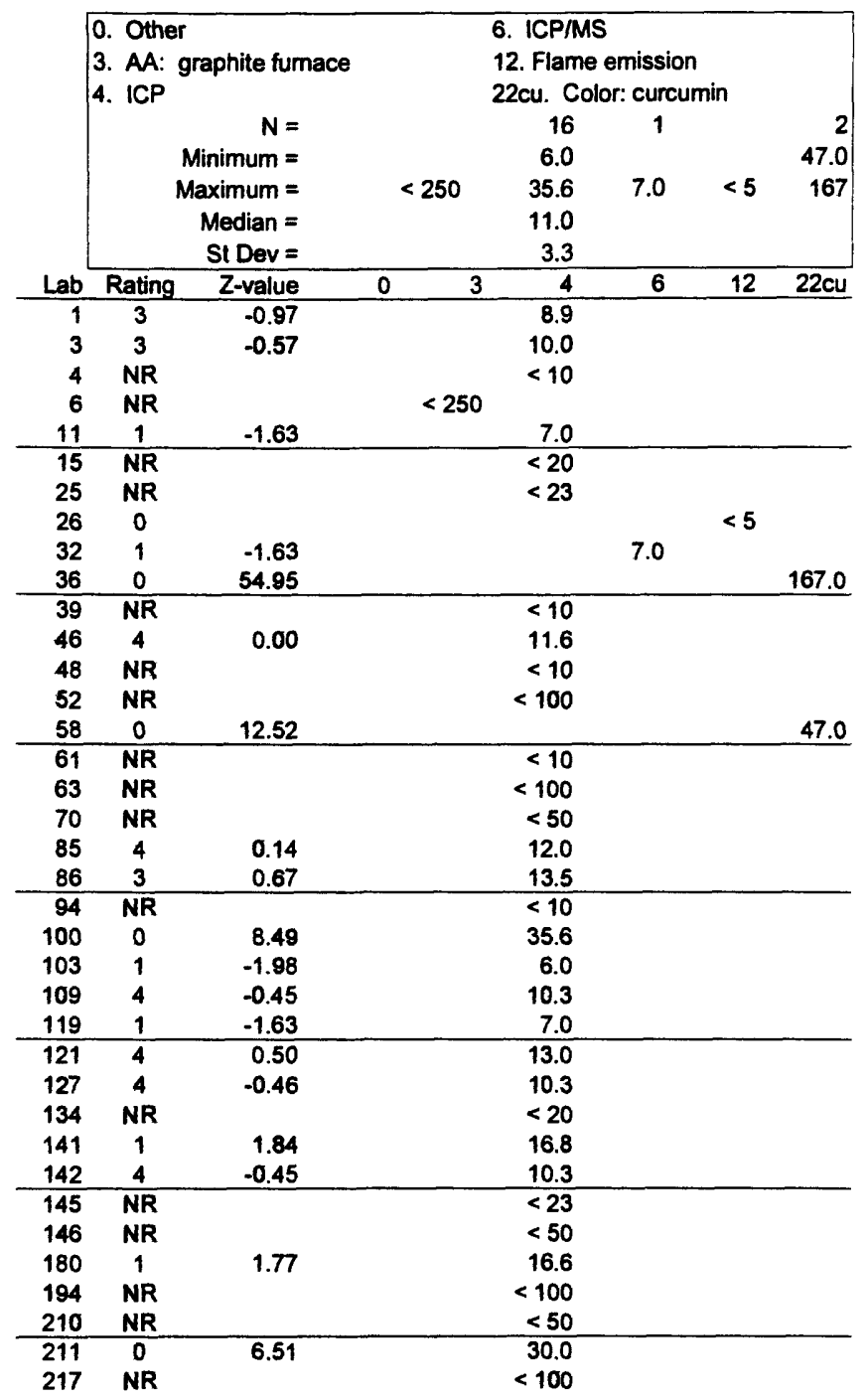


Table 10. -Statistical summary of reported data for standard reference water sample $T-129$ (trace constituents)--Continued Ba (Barium) $\mu \mathrm{g} / \mathrm{L}$

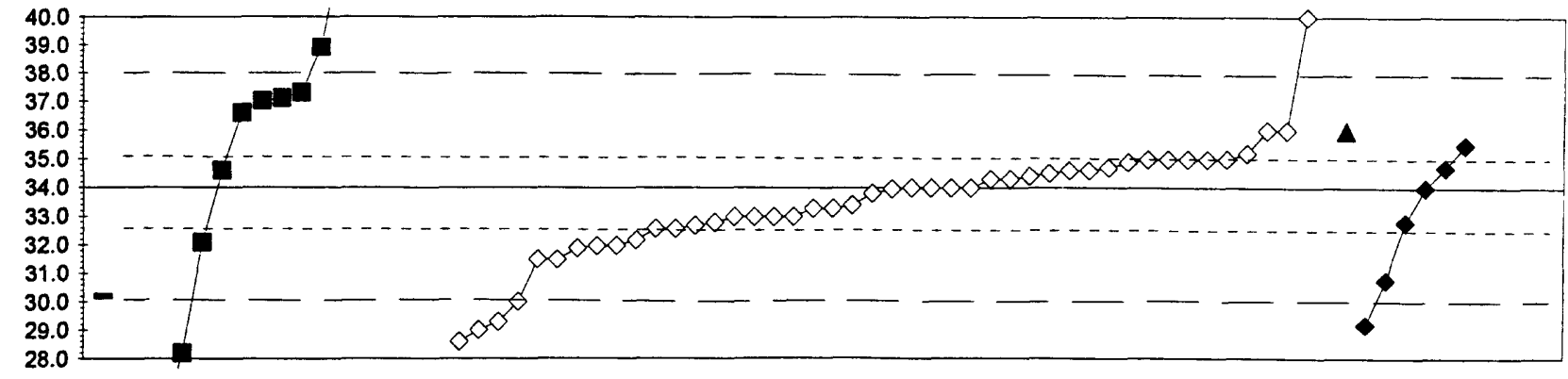

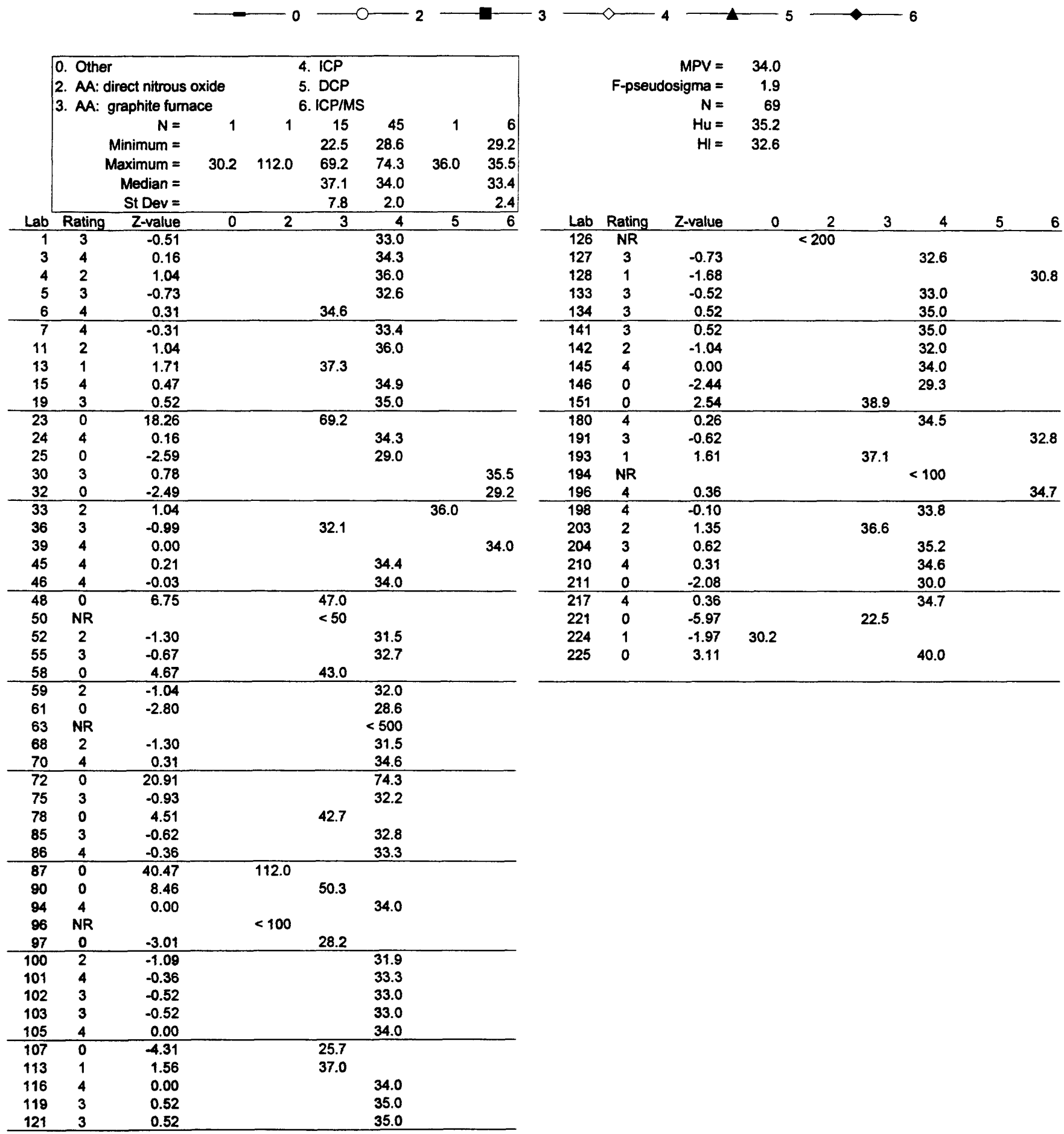


Table 10. -Statistical summary of reported data for standard reference water sample T-129 (trace constituents)--Continued Be (Beryllium) $\mu \mathrm{g} / \mathrm{L}$
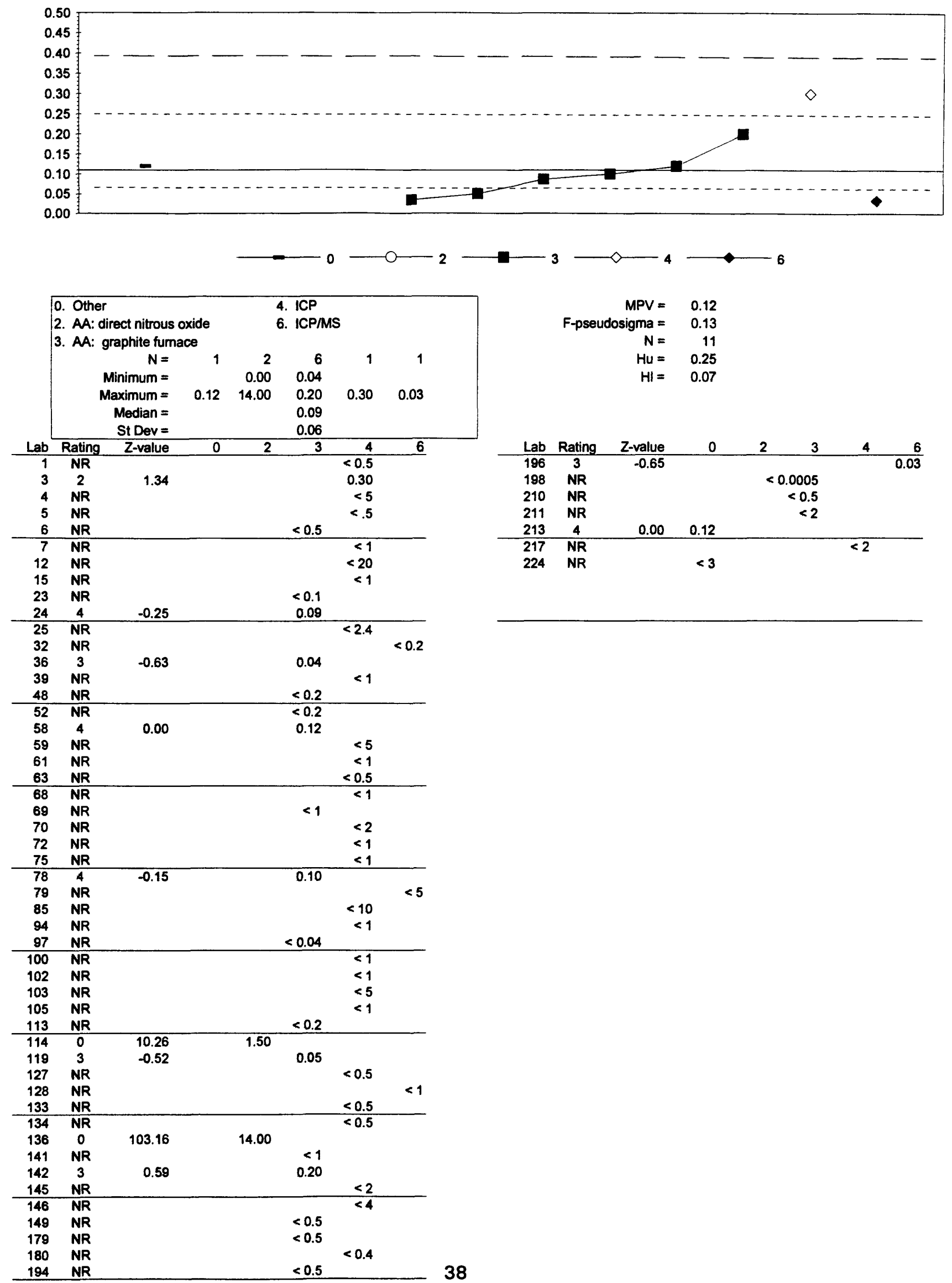
Table 10. -Statistical summary of reported data for standard reference water sample T-129 (trace constituents)--Continued Ca (Calcium) $\mathrm{mg} / \mathrm{L}$

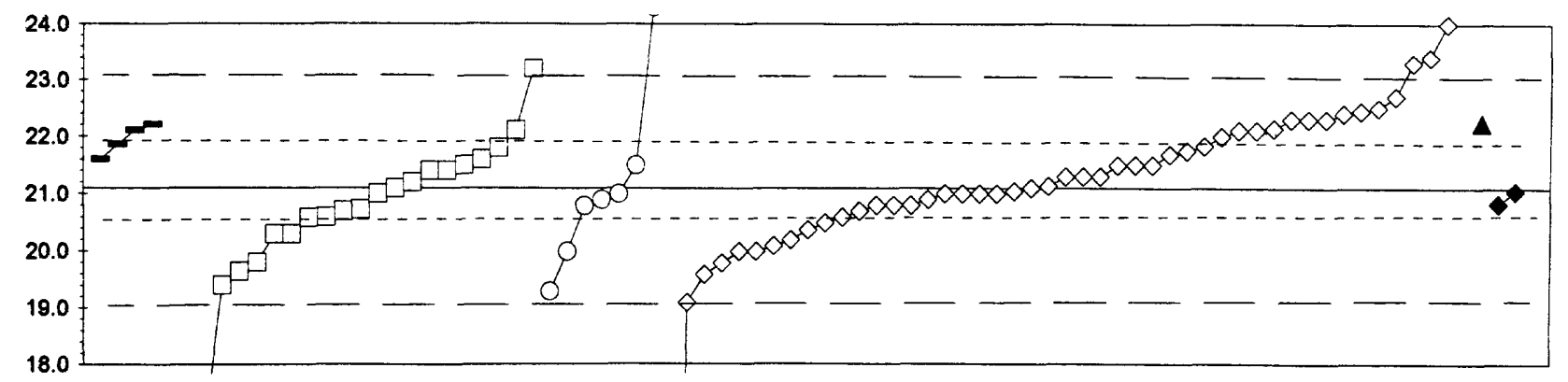

\begin{tabular}{|c|c|c|c|c|c|c|c|c|}
\hline & $\begin{array}{l}\text { 0. Other } \\
\text { 1. AA: d } \\
\text { 2. AA: d }\end{array}$ & $\begin{array}{l}\text { direct air } \\
\text { direct nitrous }\end{array}$ & xide & & $\begin{array}{l}\text { ICP } \\
\text { DCP } \\
\text { ICP/M }\end{array}$ & & & \\
\hline & & $\mathbf{N}=$ & 4 & 22 & 7 & 47 & 1 & 2 \\
\hline & & Minimum = & 21.6 & 11.0 & 19.3 & 2.2 & & 20.8 \\
\hline & & Maximum = & 22.2 & 23.2 & 24.3 & 25.6 & 22.3 & 21.1 \\
\hline & & Median = & 21.1 & 20.7 & 20.9 & 21.3 & & \\
\hline & & St Dev = & 0.3 & 1.3 & 1.6 & 1.0 & & \\
\hline Lab & Rating & Z-value & 0 & 1 & 2 & 4 & 5 & 6 \\
\hline 1 & 4 & 0.20 & & & & 21.3 & & \\
\hline 3 & 2 & 1.42 & & & & 22.5 & & \\
\hline 4 & 0 & 2.93 & & & & 24.0 & & \\
\hline 5 & 4 & -0.40 & & & & 20.7 & & \\
\hline 7 & 2 & 1.05 & & & & 22.1 & & \\
\hline 9 & 1 & -1.72 & & 19.4 & & & & \\
\hline 11 & 0 & 2.22 & & & & 23.3 & & \\
\hline 12 & 4 & -0.10 & & & & 21.0 & & \\
\hline 13 & 3 & -0.81 & & 20.3 & & & & \\
\hline 15 & 1 & 1.62 & & & & 22.7 & & \\
\hline 19 & 2 & 1.36 & & & & 22.4 & & \\
\hline 23 & 3 & -0.52 & & 20.6 & & & & \\
\hline 24 & 4 & -0.20 & & & & 20.9 & & \\
\hline 25 & 2 & -1.11 & & & & 20.0 & & \\
\hline 32 & 4 & -0.05 & & & & & & 21.1 \\
\hline 33 & 2 & 1.16 & & & & & 22.3 & \\
\hline 36 & 1 & -1.82 & & & 19.3 & & & \\
\hline 39 & 4 & 0.00 & & & & 21.1 & & \\
\hline 43 & 4 & -0.10 & & & & 21.0 & & \\
\hline 45 & 3 & 0.51 & 21.6 & & & & & \\
\hline 46 & 4 & -0.06 & & & & 21.0 & & \\
\hline 48 & 2 & 1.31 & & & & 22.4 & & \\
\hline 51 & 3 & -0.91 & & & & 20.2 & & \\
\hline 52 & 3 & -0.61 & & & & 20.5 & & \\
\hline 54 & 4 & 0.41 & & 21.5 & & & & \\
\hline 55 & 4 & 0.40 & & & & 21.5 & & \\
\hline 58 & 0 & -4.25 & & 16.9 & & & & \\
\hline 59 & 4 & -0.10 & & & & 21.0 & & \\
\hline 61 & 3 & -0.73 & & & & 20.4 & & \\
\hline 63 & 2 & -1.01 & & & & 20.1 & & \\
\hline 64 & 4 & -0.30 & & & & 20.8 & & \\
\hline 68 & 4 & 0.40 & & & & 21.5 & & \\
\hline 69 & 4 & -0.40 & & 20.7 & & & & \\
\hline 70 & 2 & 1.21 & & & & 22.3 & & \\
\hline 75 & 3 & 0.71 & & 21.8 & & & & \\
\hline 78 & 3 & -0.81 & & 20.3 & & & & \\
\hline 84 & 4 & 0.10 & & 21.2 & & & & \\
\hline 85 & 3 & -0.51 & & 20.6 & & & & \\
\hline 86 & 4 & 0.20 & & & & 21.3 & & \\
\hline 87 & 2 & -1.11 & & & 20.0 & & & \\
\hline 92 & 0 & -10.21 & & 11.0 & & & & \\
\hline 94 & 2 & 1.01 & & & & 22.1 & & \\
\hline 97 & 4 & 0.30 & & 21.4 & & & & \\
\hline 100 & 3 & -0.51 & & & & 20.6 & & \\
\hline 101 & 4 & 0.00 & & 21.1 & & & & \\
\hline 102 & 3 & 0.66 & & & & 21.8 & & \\
\hline 103 & 2 & -1.11 & & & & 20.0 & & \\
\hline 105 & 2 & 1.21 & & & & 22.3 & & \\
\hline 107 & 0 & 2.12 & & 23.2 & & & & \\
\hline 109 & 4 & -0.37 & & 20.7 & & & & \\
\hline
\end{tabular}

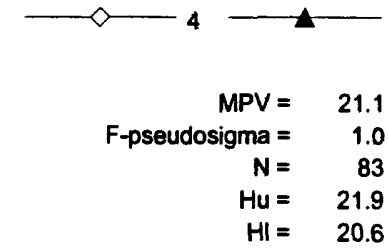

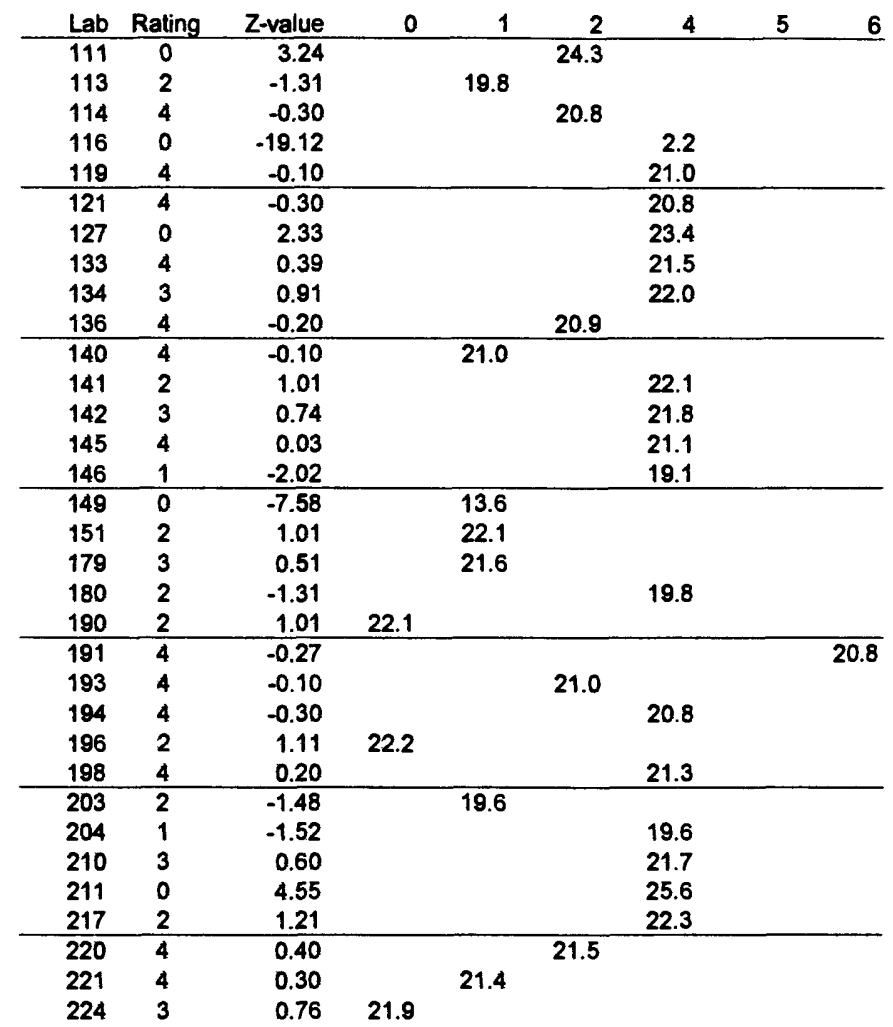


Table 10. -Statistical summary of reported data for standard reference water sample T-129 (trace constituents)--Continued Cd (Cadmium) $\mu \mathrm{g} / \mathrm{L}$

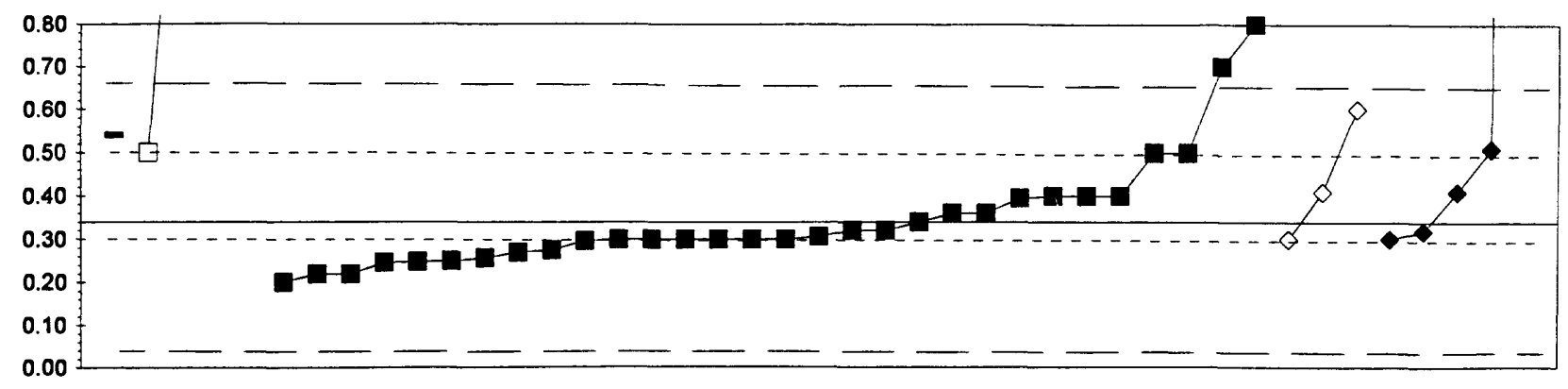

\begin{tabular}{|c|c|c|c|c|c|c|c|c|c|c|c|c|c|c|c|}
\hline \multirow[t]{2}{*}{ Lab } & \multicolumn{2}{|c|}{$\begin{array}{r}\text { 0. Other } \\
\text { 1. AA: direct air } \\
\text { 3. AA: graphite fum } \\
\text { N }= \\
\text { Minimum }= \\
\text { Maximum }= \\
\text { Median }= \\
\text { St Dev }=\end{array}$} & 1 & $\begin{array}{r}4 \\
0.50 \\
2.00\end{array}$ & $\begin{array}{r}30 \\
0.20 \\
0.80 \\
0.30 \\
0.13\end{array}$ & $\begin{array}{r}3 \\
0.30 \\
0.60\end{array}$ & \multirow[t]{2}{*}{$\begin{array}{r}5 \\
0.30 \\
11.49\end{array}$} & \multirow[b]{2}{*}{ Lab } & \multirow{2}{*}{\multicolumn{2}{|c|}{$\begin{array}{r}M P V= \\
\text { F-pseudosigma }= \\
N= \\
H u= \\
H I= \\
\text { Rating Z-value }\end{array}$}} & \multirow[t]{2}{*}{$\begin{array}{r}0.34 \\
0.15 \\
43 \\
0.50 \\
0.30 \\
\\
\\
\\
0\end{array}$} & \multirow[b]{2}{*}{1} & \multirow[b]{2}{*}{3} & \multirow[b]{2}{*}{4} & \multirow[b]{2}{*}{6} \\
\hline & Rating & Z-value & 0 & 1 & 3 & 4 & & & & & & & & & \\
\hline 1 & 4 & -0.13 & & & 0.32 & & & 114 & NR & & & $<5$ & & & \\
\hline 3 & 4 & 0.47 & & & & 0.41 & & 118 & NR & & & & $<1$ & & \\
\hline 4 & NR & & & & & $<10$ & & 119 & 4 & 0.40 & & & 0.40 & & \\
\hline 5 & 4 & -0.27 & & & 0.30 & & & 121 & 4 & -0.27 & & & 0.30 & & \\
\hline 6 & 3 & -0.63 & & & 0.25 & & & 126 & NR & & & & $<1$ & & \\
\hline 7 & NR & & & & $<0.1$ & & & 127 & 4 & -0.23 & & & 0.31 & & \\
\hline 10 & NR & & & & $<1$ & & & 128 & NR & & & & & & $<1$ \\
\hline 11 & 4 & 0.40 & & & 0.40 & & & 133 & NR & & & & & $<2$ & \\
\hline 12 & 4 & -0.27 & & & 0.30 & & & 134 & NR & & & & $<1$ & & \\
\hline 13 & NR & & & & $<1$ & & & 136 & 0 & 3.10 & & & 0.80 & & \\
\hline 15 & NR & & & & & $<10$ & & 140 & 2 & 1.08 & & 0.50 & & & \\
\hline 23 & 4 & -0.13 & & & 0.32 & & & 141 & NR & & & & $<0.5$ & & \\
\hline 24 & 2 & 1.08 & & & 0.50 & & & 142 & 2 & 1.08 & & & 0.50 & & \\
\hline 25 & NR & & & & & $<6$ & & 145 & & & & & & $<6$ & \\
\hline 26 & NR & & & & $<0.3$ & & & 146 & NR & & & & & $<5$ & \\
\hline 30 & 2 & 1.15 & & & & & 0.51 & 158 & 3 & -0.61 & & & 0.25 & & \\
\hline 32 & 4 & 0.47 & & & & & 0.41 & 179 & NR & & & & $<.5$ & & \\
\hline 36 & 3 & -0.81 & & & 0.22 & & & 180 & NR & & & & & $<2.7$ & \\
\hline 45 & 4 & 0.37 & & & 0.40 & & & 191 & 0 & 75.21 & & & & & 11.49 \\
\hline 46 & 4 & 0.00 & & & 0.34 & & & 193 & NR & & & & $<5$ & & \\
\hline 46 & 4 & -0.27 & & & 0.30 & & & 194 & NR & & & & $<1$ & & \\
\hline 50 & NR & & & & $<2$ & & & 196 & 4 & -0.15 & & & & & 0.32 \\
\hline 51 & 0 & -0.26 & & & 0.30 & & & 198 & 3 & -0.62 & & & 0.25 & & \\
\hline 52 & 4 & -0.30 & & & 0.30 & & & 203 & NR & & & & $<0.5$ & & \\
\hline 58 & 3 & -0.94 & & & 0.20 & & & 204 & NR & & & & $<1$ & & \\
\hline 59 & NR & & & & & $<5$ & & 210 & NR & & & & $<0.5$ & & \\
\hline 61 & NR & & & & & $<4$ & & 211 & 4 & -0.27 & & & 0.30 & & \\
\hline 63 & 0 & 2.43 & & & 0.70 & & & 213 & 2 & 1.35 & 0.54 & & & & \\
\hline 68 & NR & & & & & $<2$ & & 217 & NR & & & & & $<5$ & \\
\hline 69 & NR & & & & $<1$ & & & 220 & 3 & -0.81 & & & 0.22 & & \\
\hline 70 & NR & & & & $<1$ & & & 221 & 4 & -0.44 & & & 0.28 & & \\
\hline 72 & NR & & & & & $<1$ & & 225 & NR & & & & & $<5$ & \\
\hline 75 & 3 & -0.56 & & & 0.26 & & & & & & & & & & \\
\hline 76 & 4 & 0.40 & & & 0.40 & & & & & & & & & & \\
\hline 79 & NR & & & & & & $<5$ & & & & & & & & \\
\hline 85 & NR & & & $<5$ & & & & & & & & & & & \\
\hline 87 & 0 & 11.20 & & 2.00 & & & & & & & & & & & \\
\hline 90 & 4 & 0.13 & & & 0.36 & & & & & & & & & & \\
\hline 92 & 0 & 7.82 & & 1.50 & & & & & & & & & & & \\
\hline 94 & NR & & & & & $<3$ & & & & & & & & & \\
\hline 96 & NR & & & & $<2$ & & & & & & & & & & \\
\hline 97 & NR & & & & $<0.28$ & & & & & & & & & & \\
\hline 100 & 0 & 7.49 & & 1.45 & & & & & & & & & & & \\
\hline 101 & 1 & 1.75 & & & & 0.60 & & & & & & & & & \\
\hline 102 & 4 & -0.27 & & & & 0.30 & & & & & & & & & \\
\hline 103 & NR & & & & & $<5$ & & & & & & & & & \\
\hline 105 & 4 & -0.26 & & & & & 0.30 & & & & & & & & \\
\hline 107 & NR & & & & $<1$ & & & & & & & & & & \\
\hline 108 & 4 & -0.47 & & & 0.27 & & & & & & & & & & \\
\hline 113 & 4 & 0.13 & & & 0.36 & & & & & & & & & & \\
\hline
\end{tabular}


Table 10. -Statistical summary of reported data for standard reference water sample T-129 (trace constituents)--Continued Co (Cobalt) $\mu \mathrm{g} / \mathrm{L}$
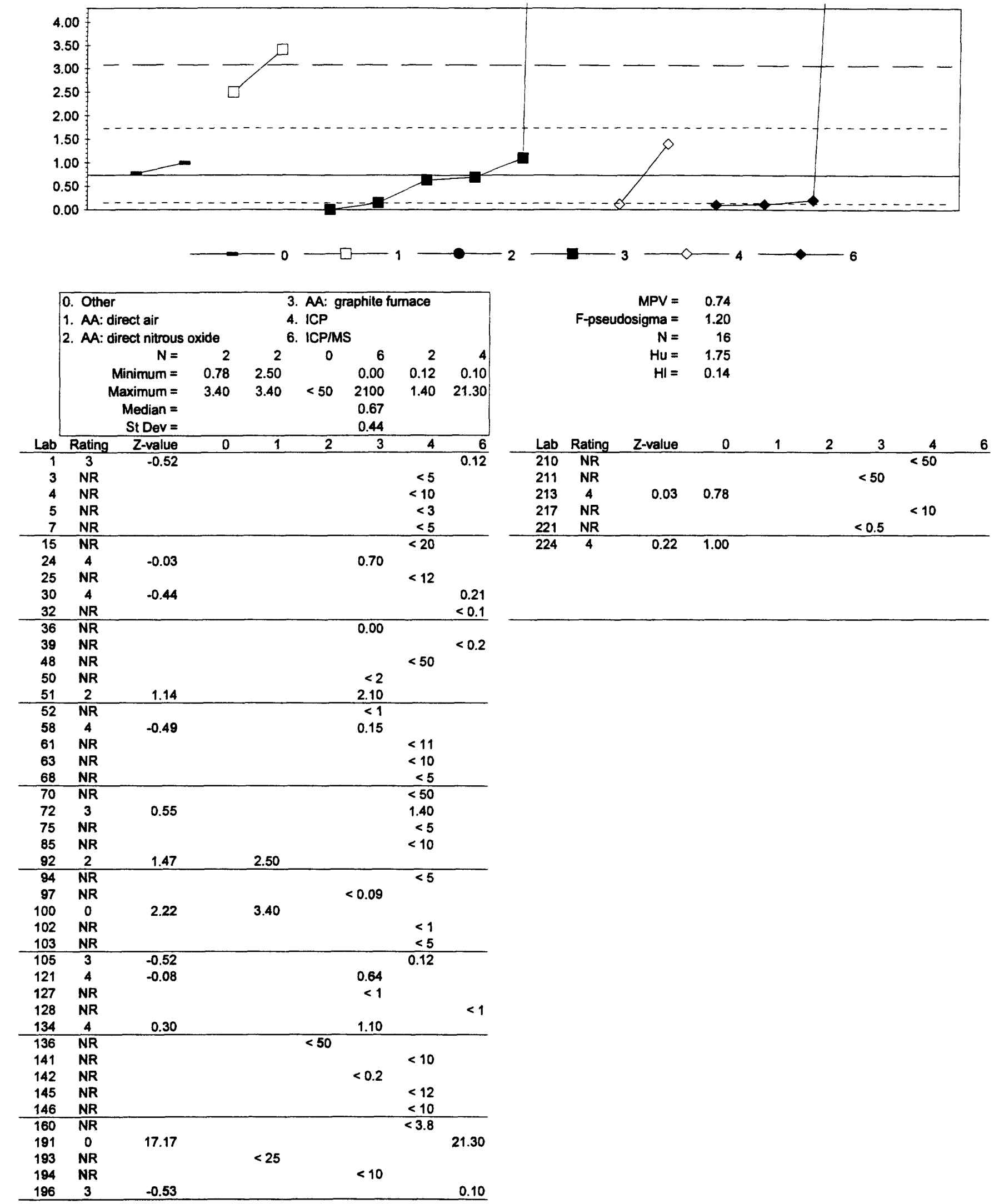
Table 10. -Statistical summary of reported data for standard reference water sample T-129 (trace constituents)--Continued Cr total (Chromium) $\mu \mathrm{g} / \mathrm{L}$
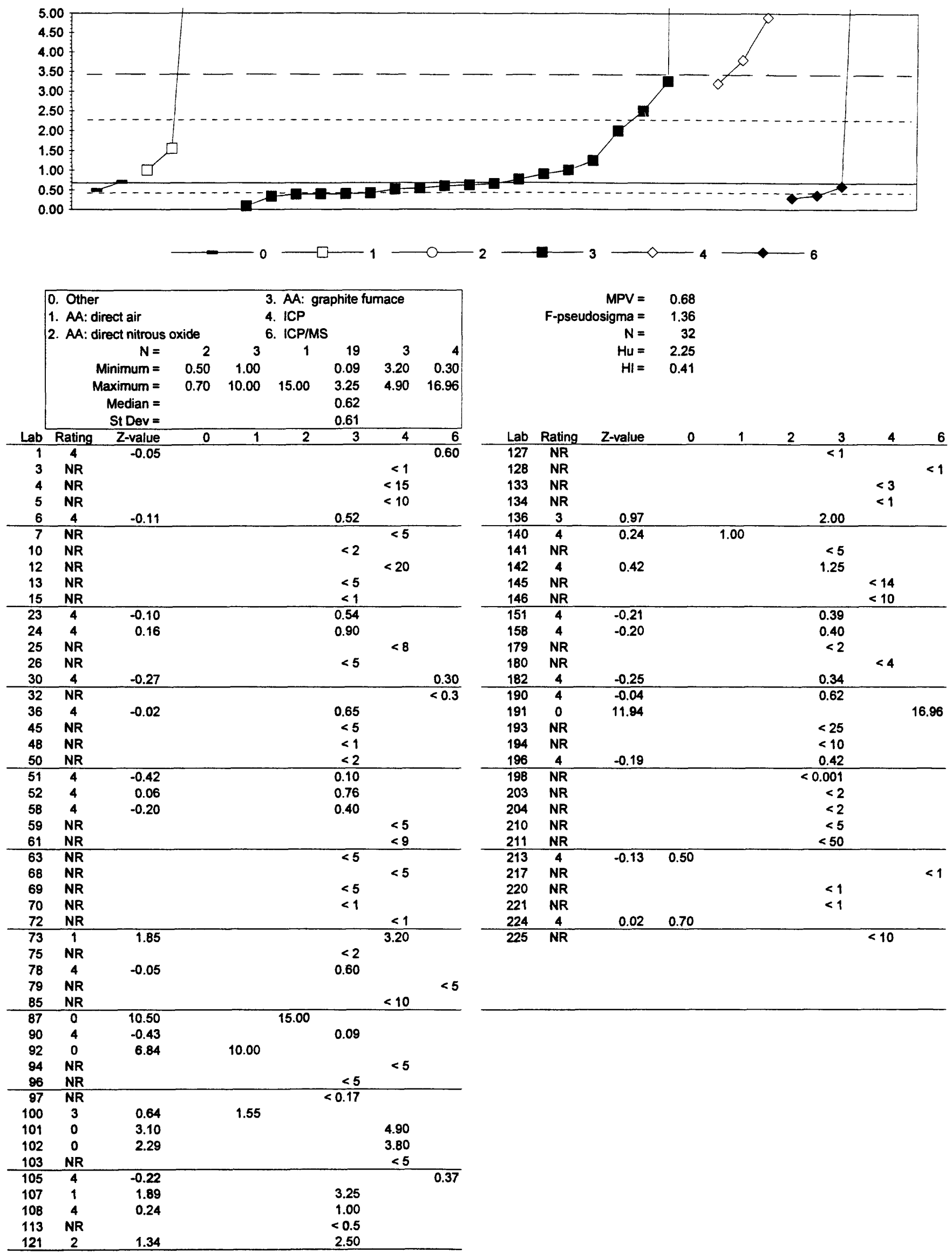
Table 10. -Statistical summary of reported data for standard reference water sample T-129 (trace constituents)--Continued Cu (Copper) $\mu \mathrm{g} / \mathrm{L}$

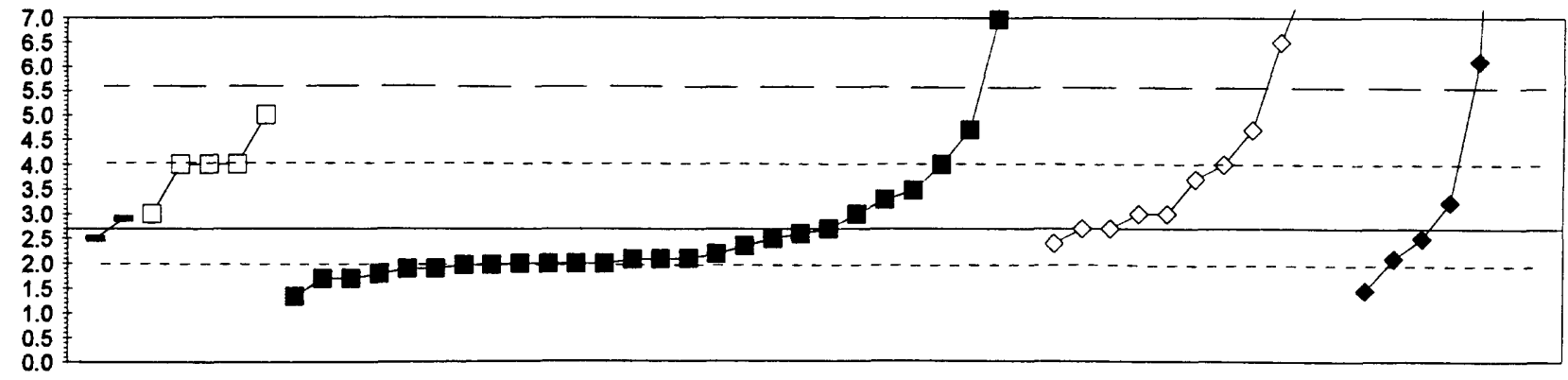

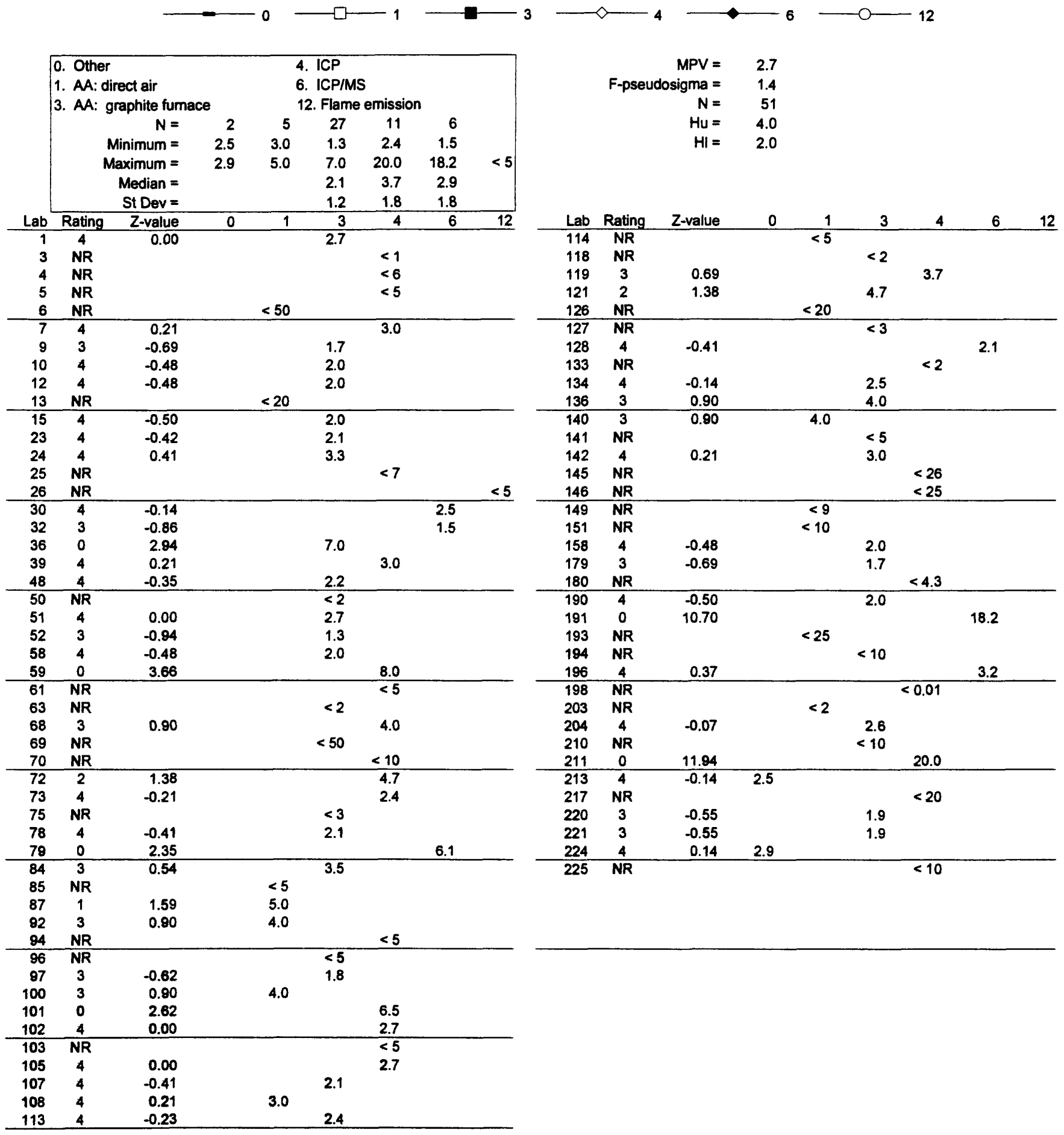


Table 10. -Statistical summary of reported data for standard reference water sample T-129 (trace constituents)-Continued Fe (Iron) $\mu \mathrm{g} / \mathrm{L}$

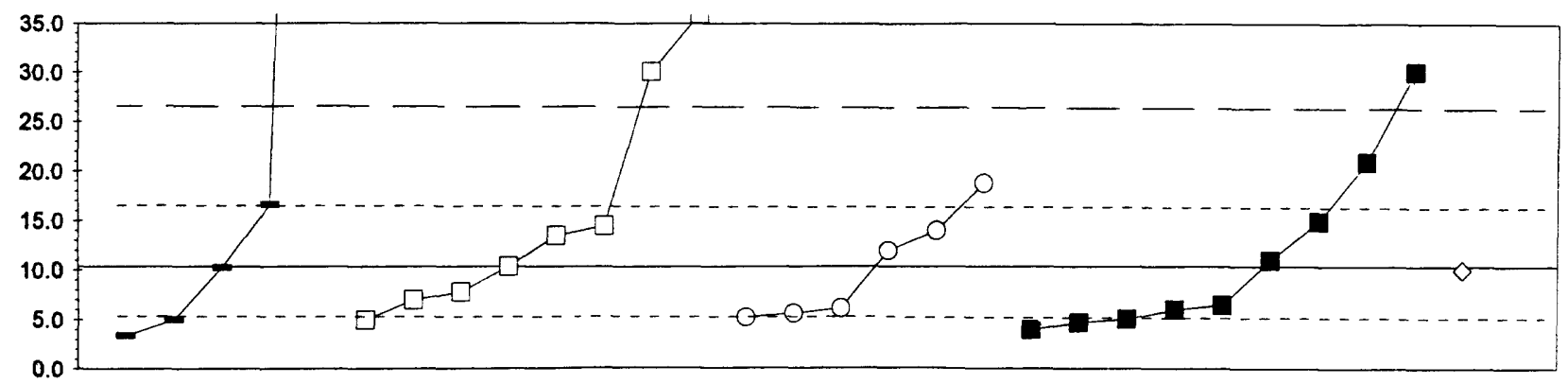

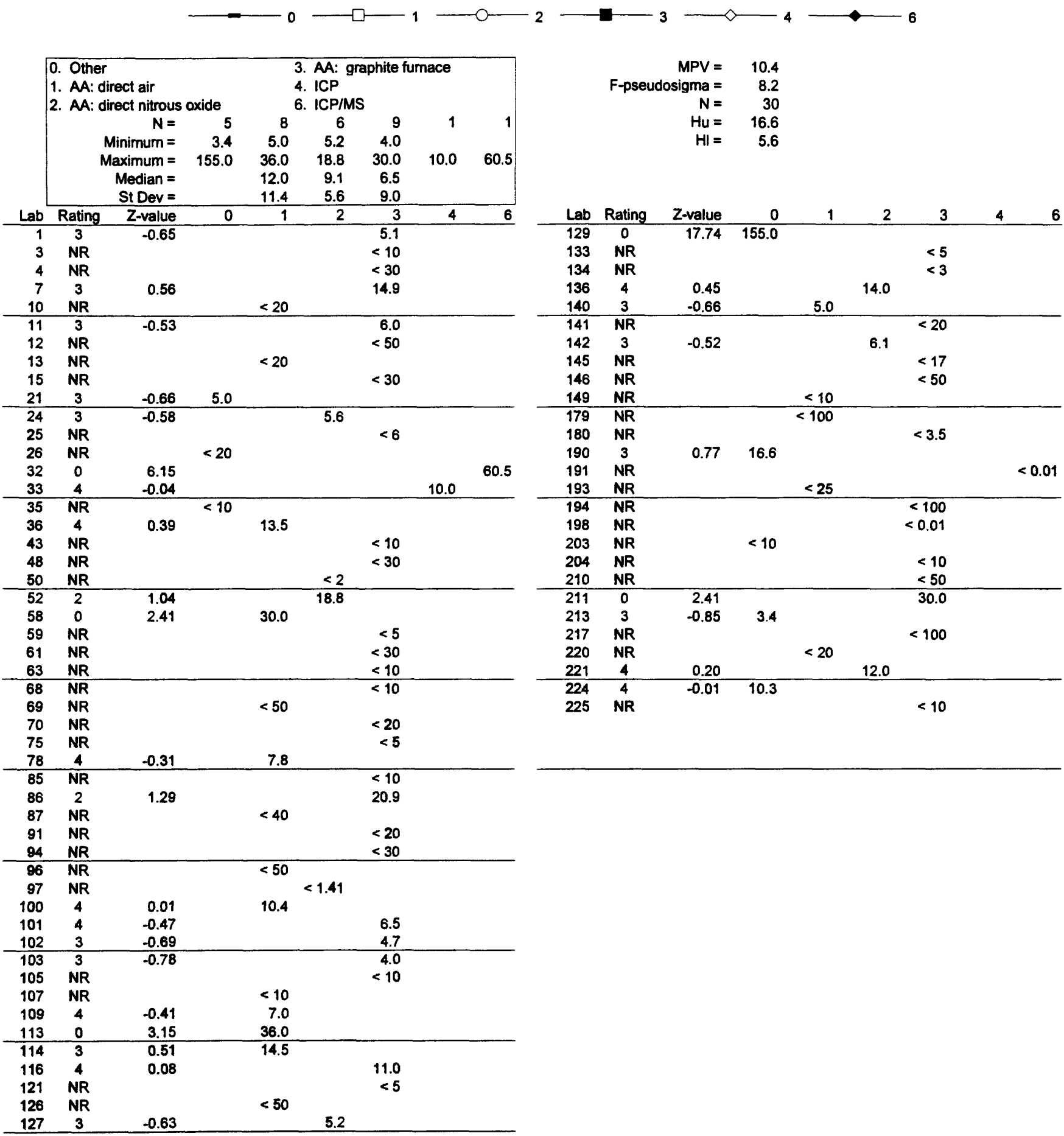


Table 10. -Statistical summary of reported data for standard reference water sample T-129 (trace constituents)--Continued K (Potassium) $\mathrm{mg} / \mathrm{L}$

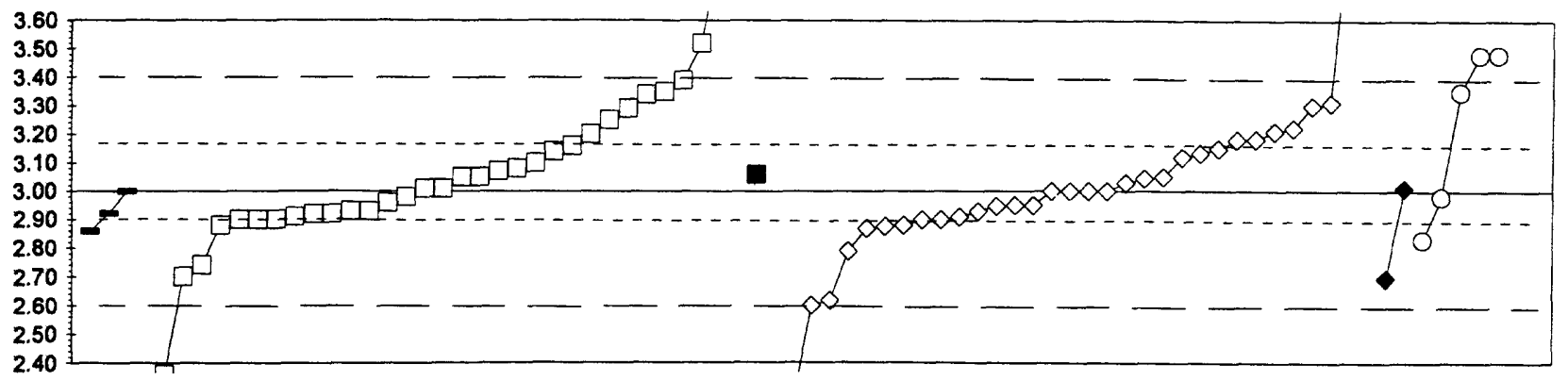

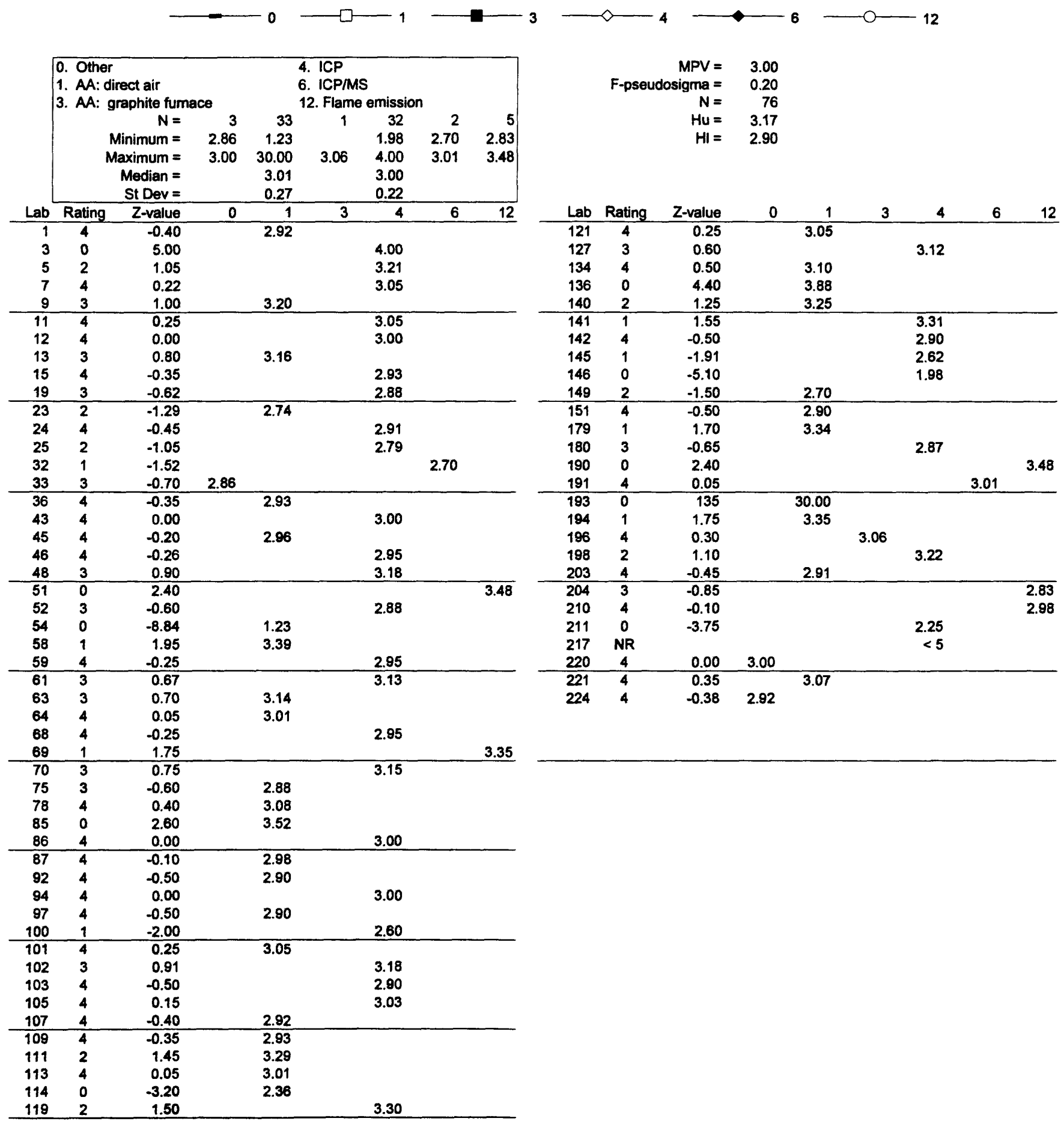


Table 10. -Statistical summary of reported data for standard reference water sample T-129 (trace constituents)--Continued Li (Lithium)

$\mu \mathrm{g} / \mathrm{L}$

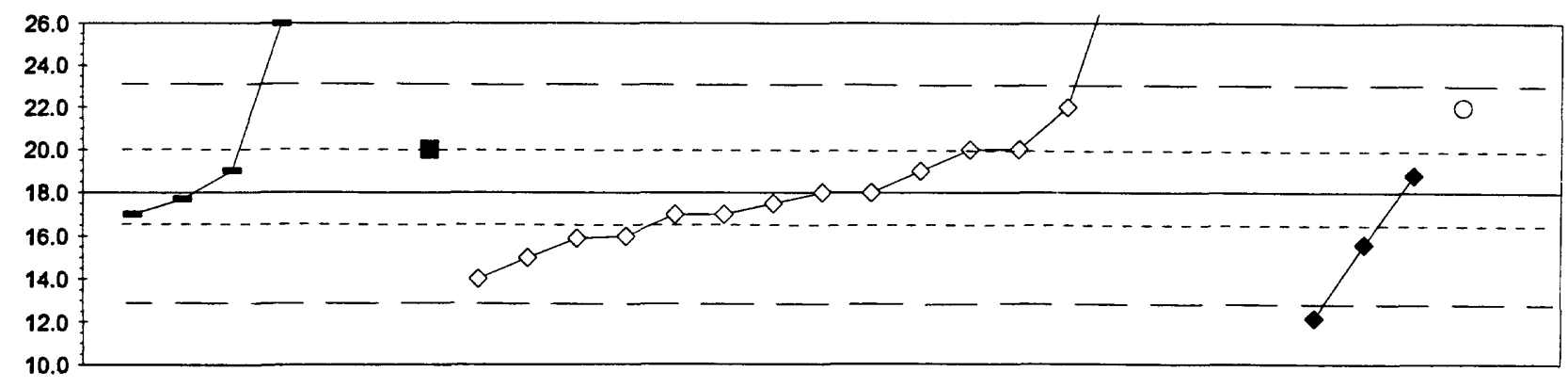

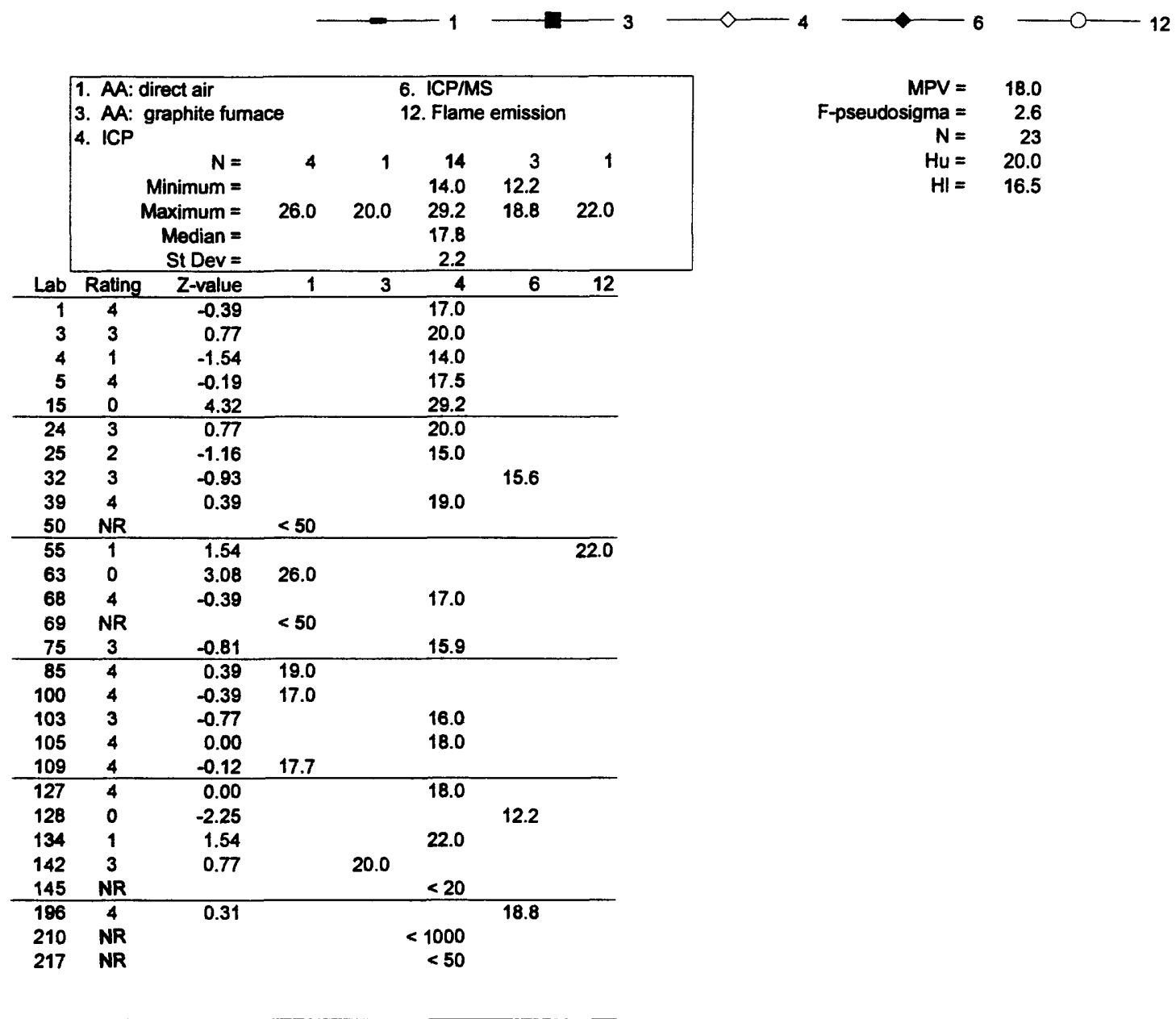


Table 10. -Statistical summary of reported data for standard reference water sample T-129 (trace constituents)-Continued Mg (Magnesium) $\mathrm{mg} / \mathrm{L}$

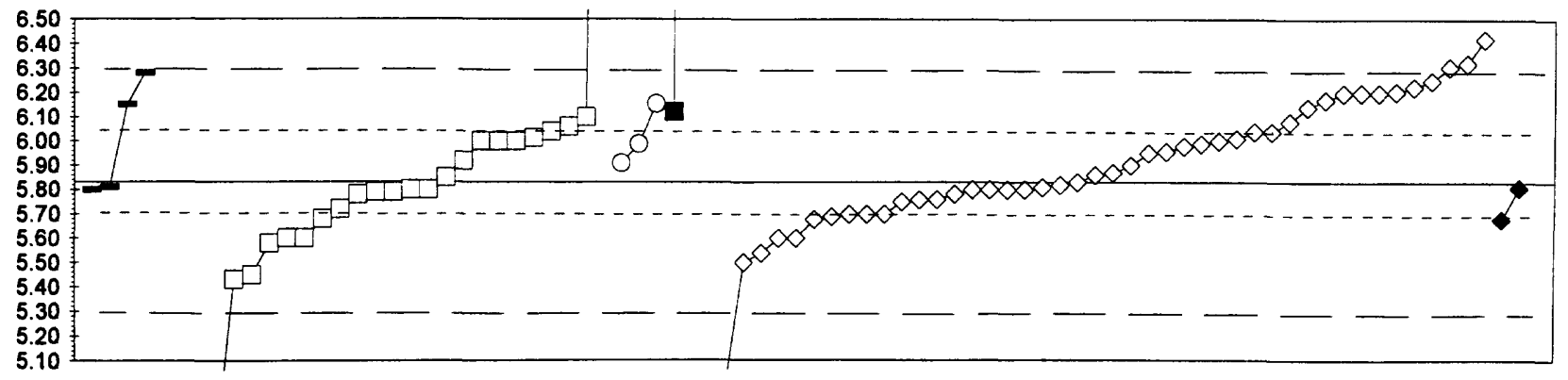

\begin{tabular}{|c|c|c|c|c|c|c|c|c|c|c|c|c|c|c|c|c|c|}
\hline \multirow[t]{2}{*}{ Lab } & \multicolumn{2}{|c|}{$\begin{array}{r}\text { 0. Other } \\
\text { 1. AA: direct air } \\
\text { 2. AA: direct nitrous } \\
\mathbb{N}= \\
\text { Minimum }= \\
\text { Maximum }= \\
\text { Median }= \\
\text { St Dev }=\end{array}$} & $\begin{array}{r}\text { xide } \\
4 \\
5.80 \\
6.28\end{array}$ & $\begin{array}{r}26 \\
2.75 \\
12.30 \\
5.79 \\
0.66 \\
\end{array}$ & $\begin{array}{l}\text { AA: } 9 \\
\text { ICP } \\
\text { ICP/M } \\
3 \\
5.91 \\
6.16\end{array}$ & $\begin{array}{r}\text { raphite f } \\
\text { S } \\
2 \\
6.12 \\
27.00\end{array}$ & $\begin{array}{r} \\
45 \\
4.93 \\
6.42 \\
5.86 \\
0.31 \\
\end{array}$ & $\begin{array}{r}2 \\
5.68 \\
5.81\end{array}$ & \multirow[b]{2}{*}{ Lab } & \multicolumn{2}{|c|}{$\begin{aligned} M P V & = \\
\text { F-pseudosigma } & = \\
N & = \\
H u & = \\
H I & =\end{aligned}$} & \multirow[t]{2}{*}{$\begin{array}{r}5.83 \\
0.25 \\
82 \\
6.04 \\
5.70 \\
\\
0\end{array}$} & \multirow[b]{2}{*}{1} & \multirow[b]{2}{*}{2} & \multirow[b]{2}{*}{3} & \multirow[b]{2}{*}{4} & \multirow[b]{2}{*}{6} \\
\hline & Rating & Z-value & 0 & 1 & 2 & 3 & 4 & 6 & & Rating & Z-value & & & & & & \\
\hline 1 & 4 & 0.38 & & 5.92 & & & & & 113 & 3 & 0.85 & & 6.04 & & & & \\
\hline 3 & 2 & 1.49 & & & & & 6.20 & & 114 & 2 & 1.31 & & & 6.16 & & & \\
\hline 4 & 2 & 1.49 & & & & & 6.20 & & 116 & 3 & 0.61 & & & & & 5.98 & \\
\hline 5 & 4 & -0.02 & & & & & 5.82 & & 119 & 4 & -0.10 & & & & & 5.80 & \\
\hline 7 & 2 & 1.50 & & & & & 6.20 & & 121 & 4 & -0.10 & & & & & 5.80 & \\
\hline 9 & 3 & -0.97 & & 5.58 & & & & & 127 & 4 & 0.02 & & & & & 5.83 & \\
\hline 11 & 1 & 1.69 & & & & & 6.25 & & 133 & 3 & 0.65 & & & & & 5.99 & \\
\hline 12 & 3 & 0.69 & & & & & 6.00 & & 134 & 4 & -0.17 & & & & & 5.78 & \\
\hline 13 & 0 & -4.90 & & 4.59 & & & & & 136 & 4 & -0.14 & & 5.79 & & & & \\
\hline 15 & 4 & 0.14 & & & & & 5.86 & & 140 & 4 & -0.10 & & 5.80 & & & & \\
\hline 19 & 1 & 1.58 & & & & & 6.22 & & 141 & 1 & 1.96 & & & & & 6.32 & \\
\hline 23 & 0 & 84.01 & & & & 27.00 & & & 142 & 2 & 1.25 & & & & & 6.14 & \\
\hline 24 & 4 & -0.06 & & & & & 5.81 & & 145 & 4 & -0.27 & & & & & 5.76 & \\
\hline 25 & 3 & -0.89 & & & & & 5.60 & & 146 & 3 & -0.89 & & & & & 5.60 & \\
\hline 32 & 3 & -0.58 & & & & & & 5.68 & 151 & 4 & 0.10 & & 5.85 & & & & \\
\hline 33 & 4 & -0.06 & 5.81 & & & & & & 179 & 3 & -0.89 & & 5.60 & & & & \\
\hline 36 & 3 & 0.93 & & 6.06 & & & & & 180 & 4 & 0.50 & & & & & 5.95 & \\
\hline 39 & 3 & 0.73 & & & & & 6.01 & & 190 & 2 & 1.29 & 6.15 & & & & & \\
\hline 43 & 4 & -0.10 & & & & & 5.80 & & 191 & 4 & -0.06 & & & & & & 5.81 \\
\hline 45 & 4 & -0.10 & 5.80 & & & & & & 193 & 3 & -0.89 & & 5.60 & & & & \\
\hline 46 & 3 & 0.52 & & & & & 5.96 & & 194 & 2 & -1.29 & & & & & 5.50 & \\
\hline 48 & 2 & 1.37 & & & & & 6.17 & & 196 & 2 & 1.17 & & & & 6.12 & & \\
\hline 51 & 3 & 0.69 & & 6.00 & & & & & 198 & 3 & 0.85 & & & & & 6.04 & \\
\hline 52 & 4 & -0.26 & & & & & 5.76 & & 203 & 4 & -0.42 & & 5.72 & & & & \\
\hline 54 & 3 & 0.75 & & 6.01 & & & & & 204 & 0 & 2.36 & & & & & 6.42 & \\
\hline 55 & 3 & -0.54 & & & & & 5.69 & & 210 & 2 & 1.48 & & & & & 6.20 & \\
\hline 58 & 0 & -4.23 & & 4.76 & & & & & 211 & 4 & -0.50 & & & & & 5.70 & \\
\hline 59 & 4 & -0.10 & & & & & 5.80 & & 217 & 1 & 1.91 & & & & & 6.31 & \\
\hline 61 & 3 & -0.58 & & & & & 5.68 & & 220 & 4 & 0.34 & & & 5.91 & & & \\
\hline 63 & 2 & -1.13 & & & & & 5.54 & & 221 & 0 & 25.69 & & 12.30 & & & & \\
\hline 68 & 4 & -0.30 & & & & & 5.75 & & 224 & 1 & 1.81 & 6.28 & & & & & \\
\hline 69 & 2 & -1.49 & & 5.45 & & & & & 225 & 0 & -3.55 & & & & & 4.93 & \\
\hline 70 & 3 & 0.85 & & & & & 6.04 & & & & & & & & & & \\
\hline 75 & 2 & 1.09 & & 6.10 & & & & & & & & & & & & & \\
\hline 78 & 0 & -6.05 & & 4.30 & & & & & & & & & & & & & \\
\hline 84 & 3 & -0.58 & & 5.68 & & & & & & & & & & & & & \\
\hline 85 & 4 & -0.18 & & 5.78 & & & & & & & & & & & & & \\
\hline 86 & 2 & 1.01 & & & & & 6.08 & & & & & & & & & & \\
\hline 87 & 1 & -1.57 & & 5.43 & & & & & & & & & & & & & \\
\hline 92 & 0 & -12.20 & & 2.75 & & & & & & & & & & & & & \\
\hline 94 & 4 & -0.50 & & & & & 5.70 & & & & & & & & & & \\
\hline 87 & 4 & -0.10 & & 5.80 & & & & & & & & & & & & & \\
\hline 100 & 4 & 0.30 & & & & & 5.90 & & & & & & & & & & \\
\hline 101 & 3 & 0.69 & & 6.00 & & & & & & & & & & & & & \\
\hline 102 & 0 & -3.27 & & & & & 5.00 & & & & & & & & & & \\
\hline 103 & 4 & -0.50 & & & & & 5.70 & & & & & & & & & & \\
\hline 105 & 4 & 0.18 & & & & & 5.87 & & & & & & & & & & \\
\hline 107 & 4 & -0.14 & & 5.79 & & & & & & & & & & & & & \\
\hline 109 & 3 & 0.69 & & 6.00 & & & & & & & & & & & & & \\
\hline 111 & 3 & 0.65 & & & 5.99 & & & & & & & & & & & & \\
\hline
\end{tabular}


Table 10. -Statistical summary of reported data for standard reference water sample $T-129$ (trace constituents)--Continued $\mathrm{Mn}$ (Manganese) $\mu \mathrm{g} / \mathrm{L}$
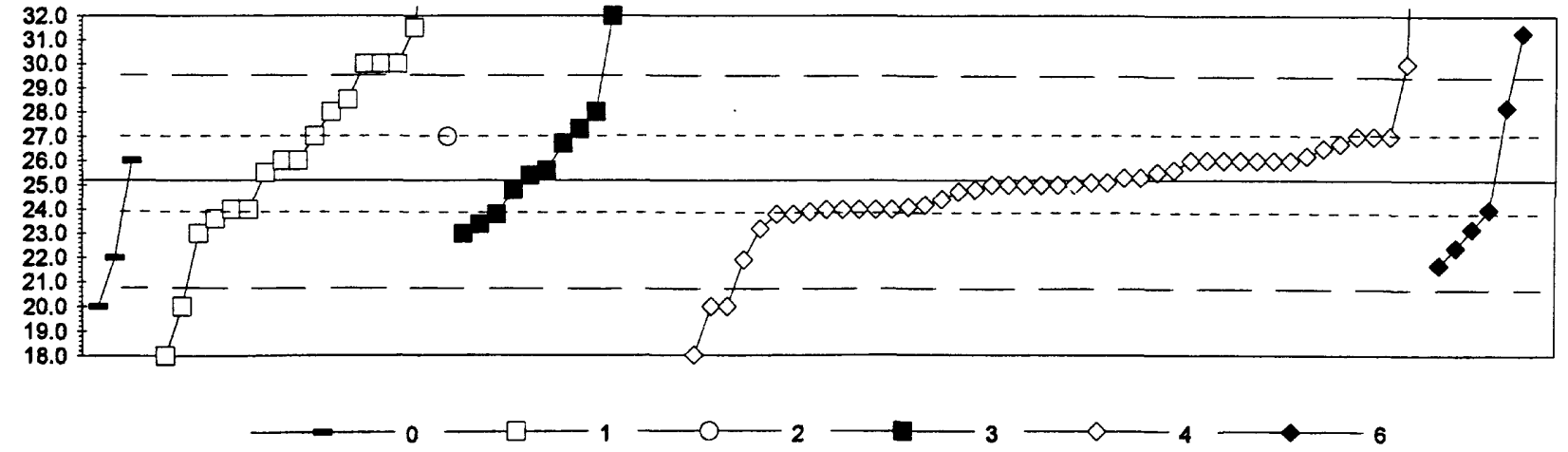

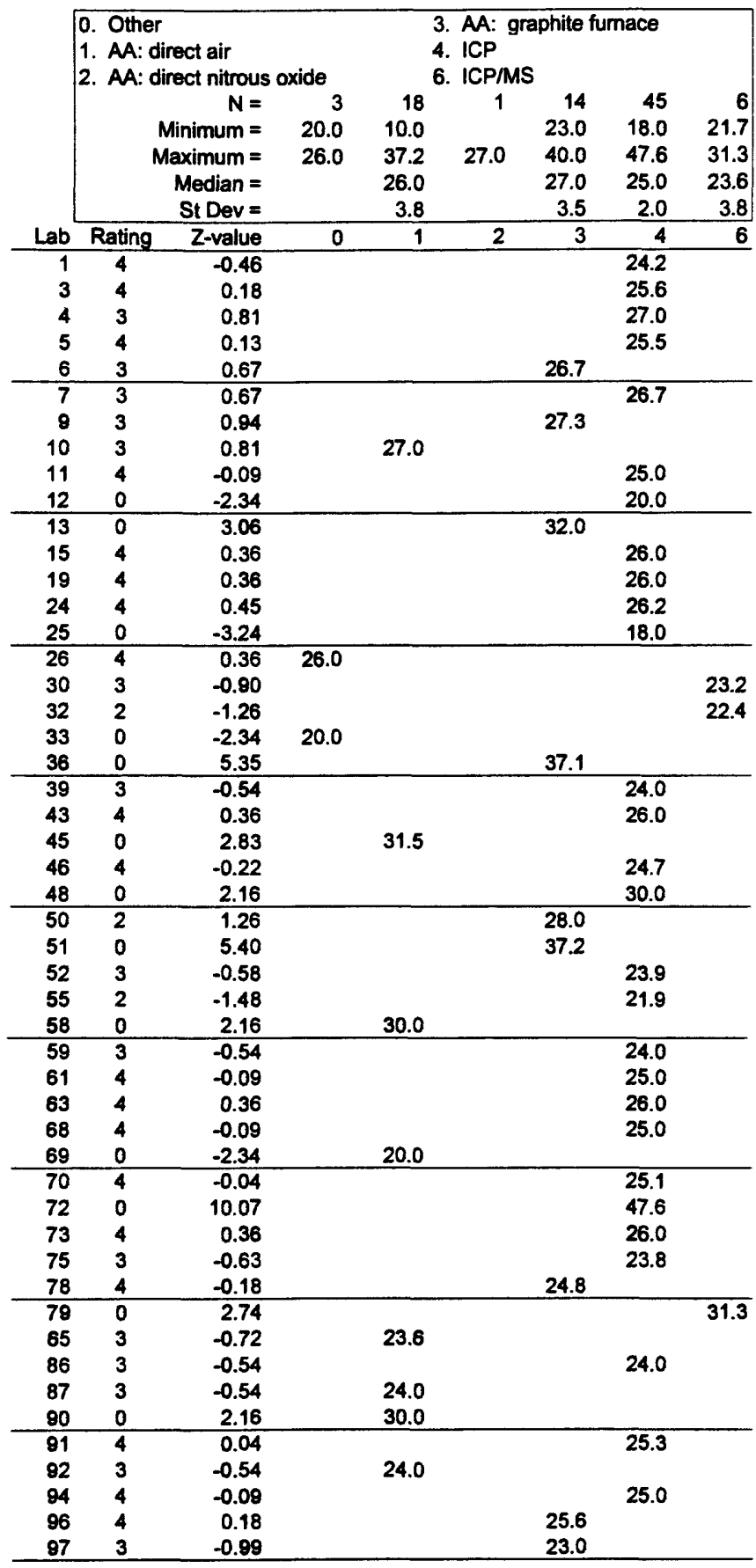

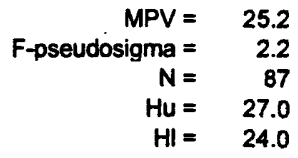

\begin{tabular}{rrrrrrrrr} 
Lab & Rating & Z-value & 0 & 1 & 2 & 3 & 4 & 6 \\
\hline 100 & 4 & 0.13 & & 25.5 & & & \\
101 & 4 & -0.18 & & & & 24.8 \\
102 & 3 & -0.63 & & & & 23.8 \\
103 & 4 & 0.36 & & & & 26.0 \\
105 & 3 & -0.54 & & & & 24.0 & \\
\hline 107 & 0 & 2.16 & & 30.0 & & &
\end{tabular}

109

1119

$\begin{array}{lll}109 & 2 & 1.26 \\ 113 & 4 & 0.09\end{array}$

\begin{tabular}{rrrrr}
114 & 2 & 1.48 & 28.5 & \\
116 & 3 & 0.81 & & 27.0 \\
\hline 119 & 4 & -0.09 & & 25.0 \\
121 & 4 & 0.36 & & 26.0
\end{tabular}

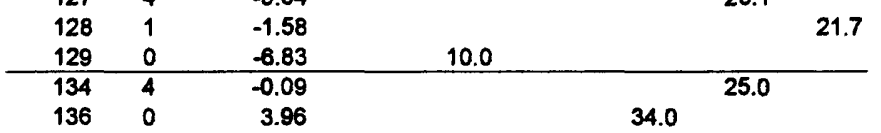

\begin{tabular}{l}
141 \\
142 \\
\hline 145
\end{tabular}

\begin{tabular}{|c|c|c|c|c|}
\hline 142 & $\begin{array}{l}3 \\
4\end{array}$ & $\begin{array}{l}0.81 \\
0.04\end{array}$ & & $\begin{array}{l}27.0 \\
25.3\end{array}$ \\
\hline 145 & 3 & -0.54 & & 24.0 \\
\hline 146 & 3 & -0.90 & & 23.2 \\
\hline 149 & 4 & 0.36 & 26.0 & \\
\hline
\end{tabular}

$\begin{array}{lll}149 & 4 & 0.36 \\ 151 & 0 & 5.40\end{array}$

\begin{tabular}{lll}
179 & 0 & -3.24 \\
\hline 180 & 4 & -0.36
\end{tabular}

18.0

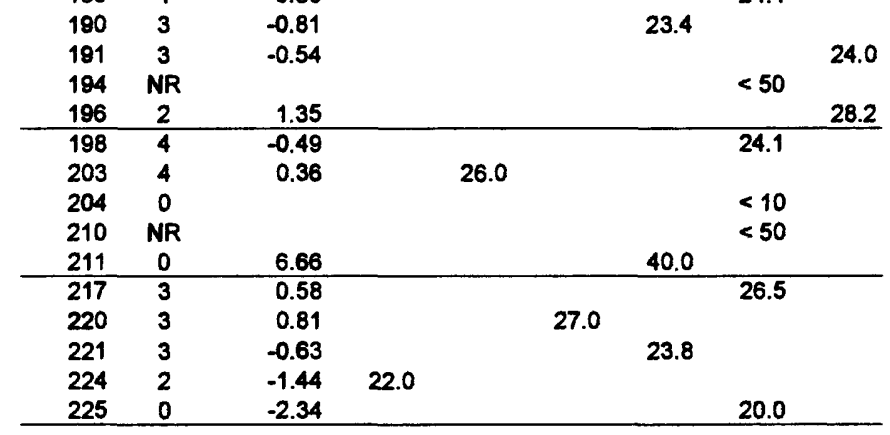


Table 10. -Statistical summary of reported data for standard reference water sample T-129 (trace constituents)--Continued Mo (Molybdenum) $\mu \mathrm{g} / \mathrm{L}$

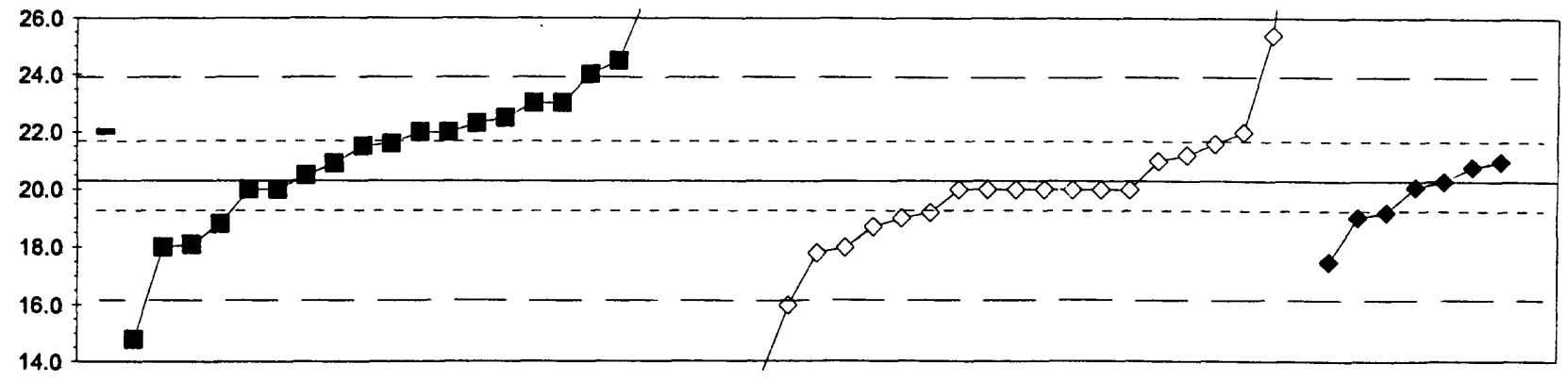

\begin{tabular}{|c|c|c|c|c|c|c|}
\hline \multirow[b]{2}{*}{ Lab } & $\begin{array}{l}\text { 0. Other } \\
\text { 3. AA: } 9 \\
\text { 4. ICP }\end{array}$ & 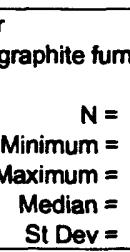 & $\begin{array}{r}1 \\
22.0\end{array}$ & $\begin{array}{r}21 \\
14.8 \\
38.5 \\
22.0 \\
2.7 \\
\end{array}$ & $\begin{array}{r}20 \\
13.3 \\
33.6 \\
20.0 \\
2.0 \\
\end{array}$ & $\begin{array}{r}7 \\
17.5 \\
21.0 \\
20.1 \\
1.2\end{array}$ \\
\hline & Rating & Z-value & 0 & 3 & 4 & 6 \\
\hline 1 & 3 & -0.53 & & & & 19.2 \\
\hline 3 & 2 & -1.11 & & & 18.0 & \\
\hline 4 & NR & & & & $<50$ & \\
\hline 5 & 0 & -3.37 & & & 13.3 & \\
\hline 6 & 1 & 2.02 & & 24.5 & & \\
\hline 7 & 0 & 2.46 & & & 25.4 & \\
\hline 11 & 0 & -2.07 & & & 16.0 & \\
\hline 12 & NR & & & & $<30$ & \\
\hline 15 & 3 & 0.96 & & 22.3 & & \\
\hline 23 & 1 & 1.78 & & 24.0 & & \\
\hline 24 & 4 & -0.14 & & & 20.0 & \\
\hline 30 & 4 & 0.00 & & & & 20.3 \\
\hline 32 & 2 & -1.35 & & & & 17.5 \\
\hline 36 & 2 & -1.06 & & 18.1 & & \\
\hline 39 & 4 & 0.34 & & & 21.0 & \\
\hline 45 & 0 & 3.23 & & 27.0 & & \\
\hline 48 & NR & & & & $<100$ & \\
\hline 50 & 4 & -0.14 & & 20.0 & & \\
\hline 52 & 4 & 0.29 & & 20.9 & & \\
\hline 58 & 4 & -0.14 & & 20.0 & & \\
\hline 61 & 3 & -0.53 & & & 19.2 & \\
\hline 63 & 3 & 0.82 & & 22.0 & & \\
\hline 68 & 3 & 0.82 & & & 22.0 & \\
\hline 70 & NR & & & & $<50$ & \\
\hline 72 & 0 & 6.41 & & & 33.6 & \\
\hline 75 & 4 & -0.14 & & & 20.0 & \\
\hline 78 & 3 & 0.63 & & 21.6 & & \\
\hline 79 & 4 & -0.10 & & & & 20.1 \\
\hline 85 & 4 & 0.43 & & & 21.2 & \\
\hline 86 & 3 & 0.63 & & & 21.6 & \\
\hline 87 & 2 & 1.06 & & 22.5 & & \\
\hline 94 & 2 & -1.11 & & 18.0 & & \\
\hline 97 & 4 & 0.10 & & 20.5 & & \\
\hline 100 & NR & & & & $<\mathbf{5 0}$ & \\
\hline 103 & 4 & -0.14 & & & 20.0 & \\
\hline 105 & 4 & 0.24 & & & & 20.8 \\
\hline 109 & 3 & 0.82 & & 22.0 & & \\
\hline 121 & 4 & -0.14 & & & 20.0 & \\
\hline 127 & 2 & 1.30 & & 23.0 & & \\
\hline 128 & 3 & -0.61 & & & & 19.0 \\
\hline 134 & 4 & -0.14 & & & 20.0 & \\
\hline 141 & 3 & -0.63 & & & 19.0 & \\
\hline 142 & 0 & 8.77 & & 38.5 & & \\
\hline 145 & 4 & -0.14 & & & 20.0 & \\
\hline 146 & 2 & -1.20 & & & 17.8 & \\
\hline 151 & 3 & -0.72 & & 18.8 & & \\
\hline 179 & 0 & 5.49 & & 31.7 & & \\
\hline 180 & 3 & -0.77 & & & 18.7 & \\
\hline 182 & 3 & 0.58 & & 21.5 & & \\
\hline 194 & 2 & 1.30 & & 23.0 & & \\
\hline
\end{tabular}

\begin{tabular}{rrrrrrr} 
Lab & Rating & Z-value & 0 & 3 & 4 & 6 \\
\hline 196 & 4 & 0.34 & & & & 21.0 \\
198 & 0 & & & & $<0.05$ & \\
210 & NR & & & & $<50$ & \\
211 & NR & & & $<100$ & & \\
217 & 4 & -0.14 & & & 20.0 & \\
\hline 221 & 0 & -2.65 & & 14.8 & & \\
224 & 3 & 0.82 & 22.0 & & & \\
225 & NR & & & & $<20$ &
\end{tabular}


Table 10. -Statistical summary of reported data for standard reference water sample T-129 (trace constituents)-Continued $\mathrm{Na}$ (Sodium) $\mathrm{mg} / \mathrm{L}$

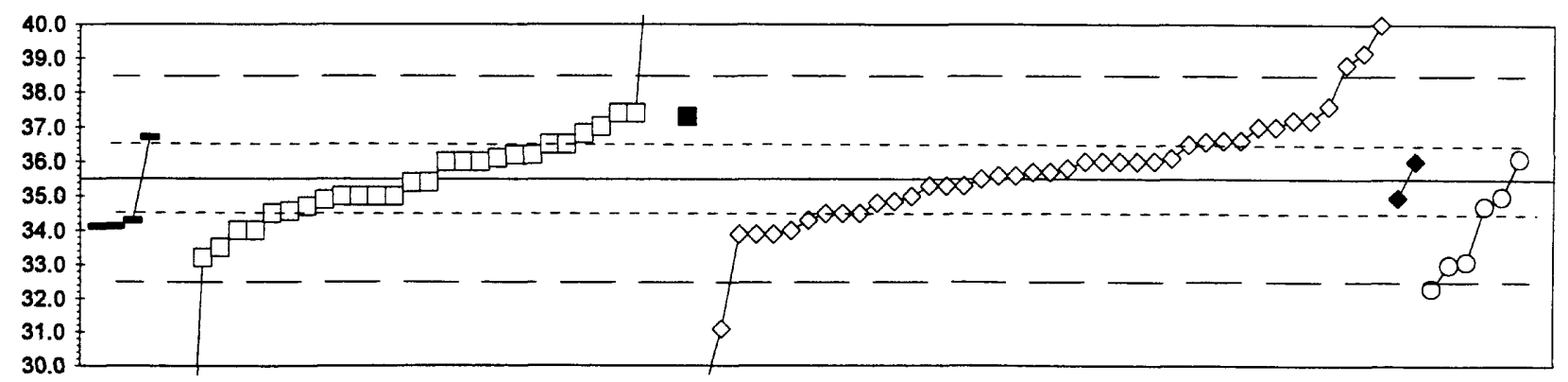

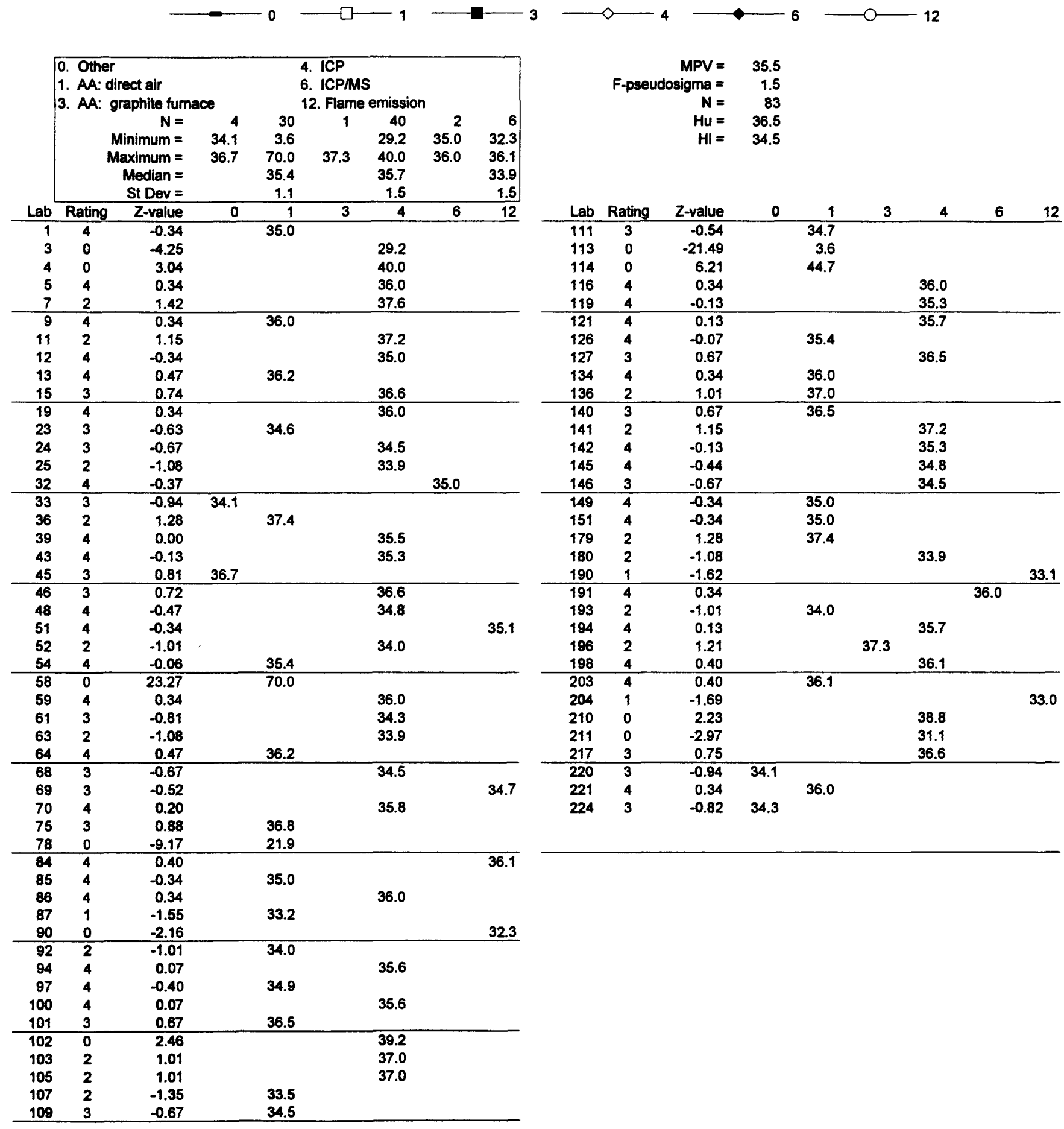


Table 10. -Statistical summary of reported data for standard reference water sample T-129 (trace constituents)--Continued $\mathrm{Ni}$ (Nickel) $\mu \mathrm{g} / \mathrm{L}$
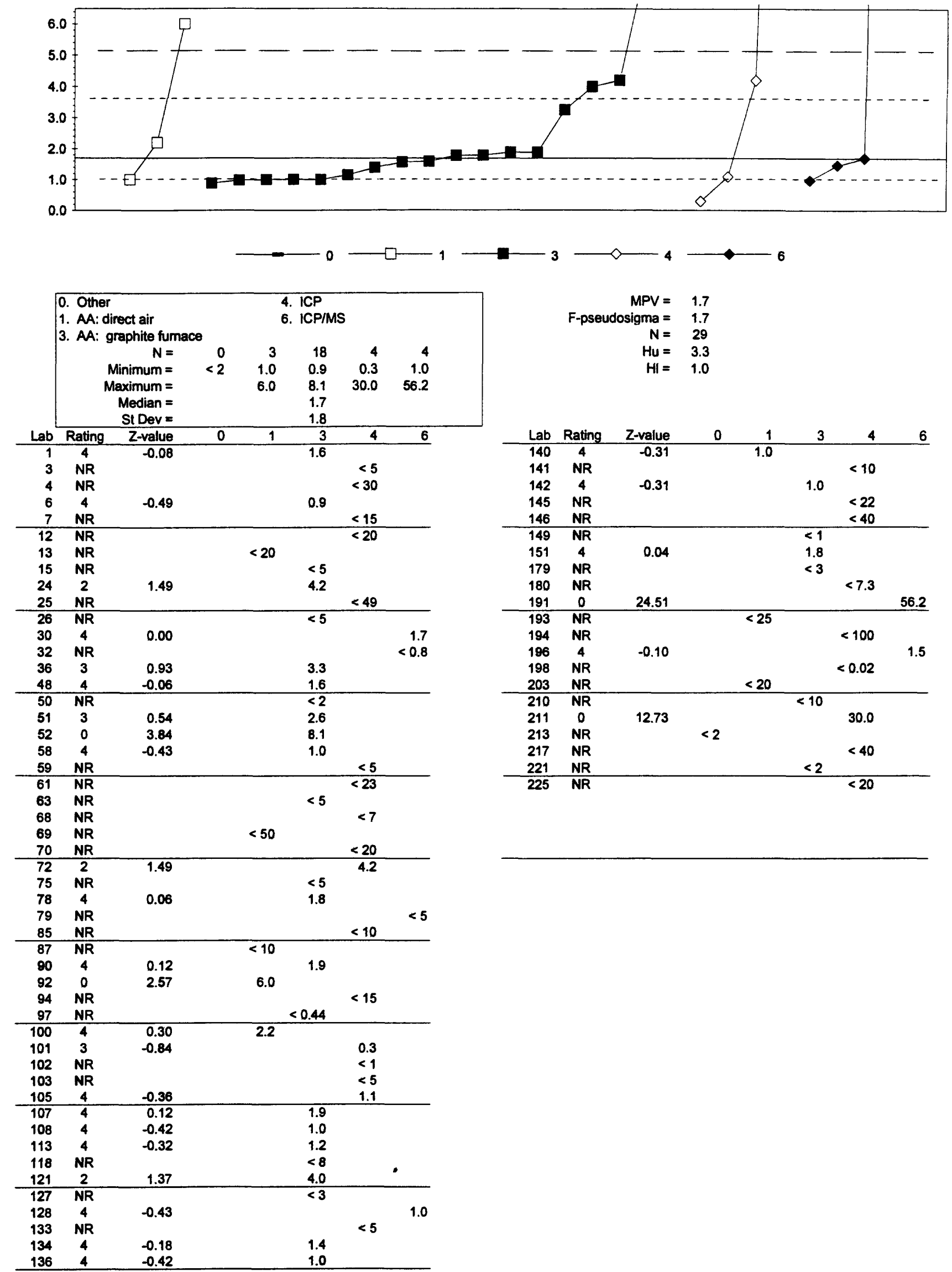
Table 10. -Statistical summary of reported data for standard reference water sample T-129 (trace constituents)-Continued $\mathrm{Pb}$ (Lead) $\mu \mathrm{g} / \mathrm{L}$

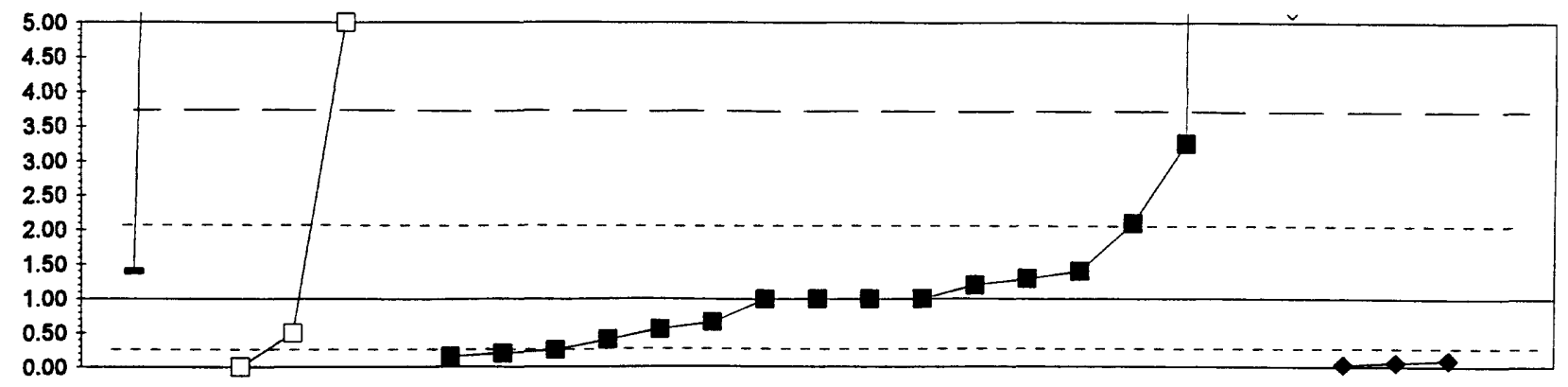

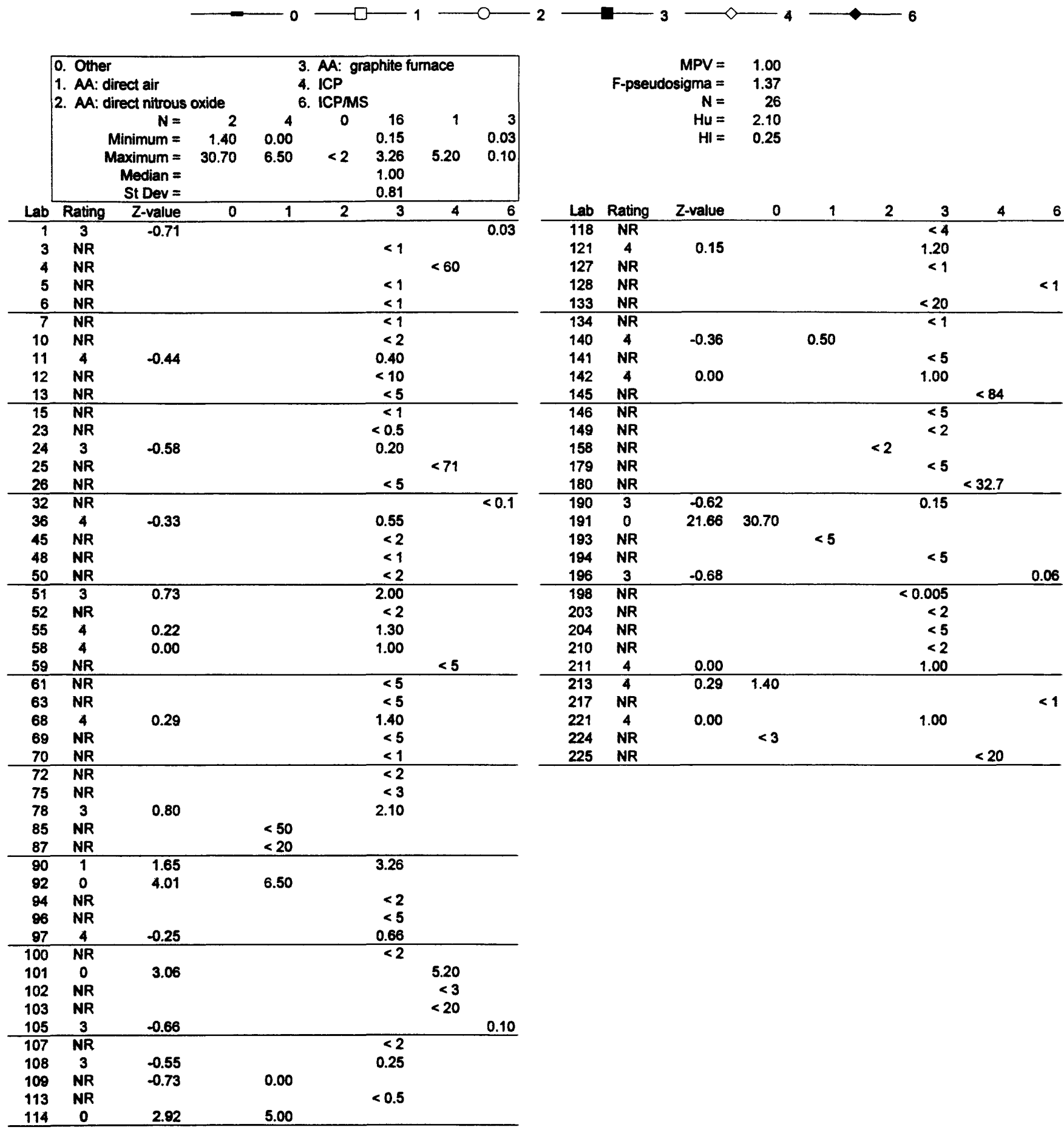


Table 10. -Statistical summary of reported data for standard reference water sample $T-129$ (trace constituents)--Continued $\mathrm{Sb}$ (Antimony) $\quad \mu \mathrm{g} / \mathrm{L}$
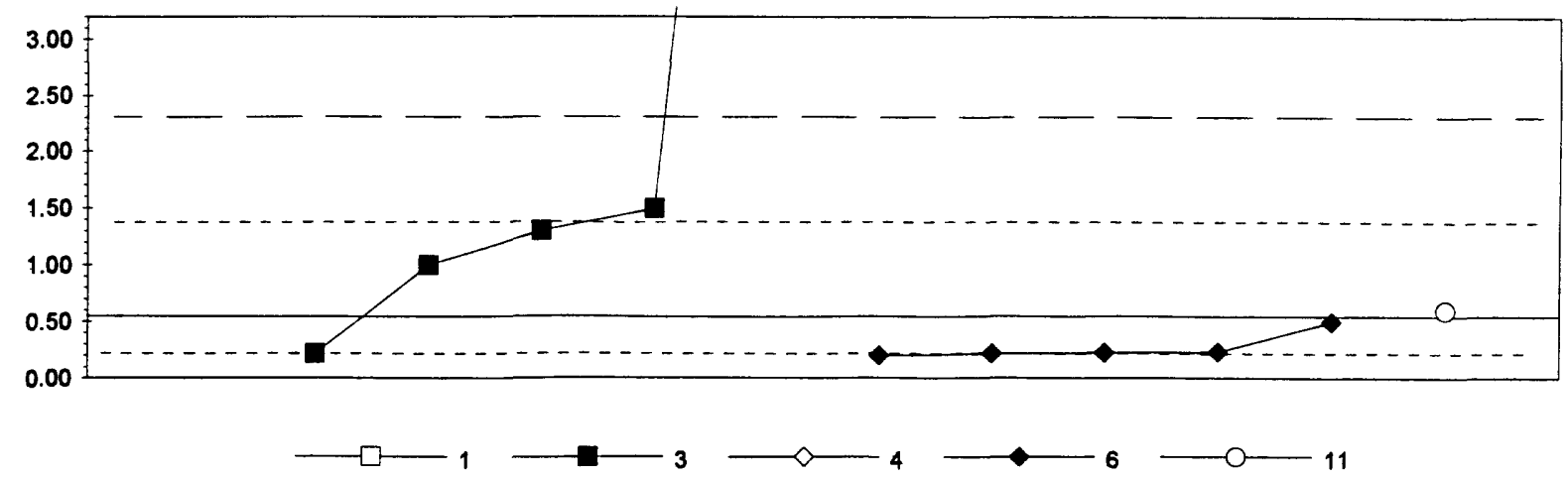

\begin{tabular}{|c|c|c|c|c|c|c|c|}
\hline \multirow[b]{3}{*}{ Lab } & \multicolumn{3}{|c|}{$\begin{array}{l}\text { 1. AA: direct air } \\
\text { 3. AA: graphite fumace } \\
\text { 4. ICP }\end{array}$} & \multicolumn{3}{|c|}{$\begin{array}{l}\text { 6. ICP/MS } \\
\text { 11. AA: hydride }\end{array}$} & \multirow[b]{2}{*}{$\begin{array}{r}1 \\
0.60\end{array}$} \\
\hline & $\left.\right|^{7} \cdot 1$ & $\begin{aligned} N & = \\
\text { Minimum } & = \\
\text { Maximum } & = \\
\text { Median } & = \\
\text { St Dev } & =\end{aligned}$ & $\begin{array}{r}1 \\
180\end{array}$ & $\begin{array}{r}5 \\
0.22 \\
11.00 \\
1.30 \\
0.56 \\
\end{array}$ & $\begin{array}{r}0 \\
<1 \\
<100\end{array}$ & $\begin{array}{r}5 \\
0.20 \\
0.50 \\
0.23 \\
0.13 \\
\end{array}$ & \\
\hline & Rating & Z-value & $\overline{1}$ & 3 & 4 & 6 & 11 \\
\hline 1 & 4 & -0.38 & & & & & \\
\hline 3 & NR & & & $<2$ & & & \\
\hline 6 & NR & & & $<3$ & & & \\
\hline 7 & NR & & & & $<26$ & & \\
\hline 12 & NR & & & & $<100$ & & \\
\hline 15 & NR & & & & $<50$ & & \\
\hline 23 & NR & & & $<2$ & & & \\
\hline 24 & 3 & 0.52 & & 1.00 & & & \\
\hline 25 & NR & & & & $<51$ & & \\
\hline 30 & 4 & -0.06 & & & & & \\
\hline 32 & NR & & & & & - & \\
\hline 36 & 4 & -0.38 & & 0.22 & & & \\
\hline 39 & 4 & -0.40 & & & & & \\
\hline 45 & NR & & & $<5$ & & & \\
\hline 48 & NR & & & $<3$ & & & \\
\hline 52 & NR & & & $<6$ & & & \\
\hline 58 & 0 & 11.99 & & 11.00 & & & \\
\hline 61 & NR & & & & $<26$ & & \\
\hline 63 & NR & & & $<5$ & & & \\
\hline 68 & NR & & & $<0.5$ & & & \\
\hline 69 & NR & & & $<5$ & & & \\
\hline 70 & NR & & & $<5$ & & & \\
\hline 75 & NR & & & & $<50$ & & \\
\hline 78 & 3 & 0.86 & & 1.30 & & & \\
\hline 85 & NR & & & & $<100$ & & \\
\hline 100 & NR & & & $<2$ & & & \\
\hline 102 & NR & & & & $<1$ & & \\
\hline 105 & 4 & -0.36 & & & & & \\
\hline 113 & NR & & & $<2.2$ & & & \\
\hline 114 & 0 & 205 & 180 & & & & \\
\hline 119 & 4 & 0.06 & & & & & \\
\hline 127 & NR & & & $<2$ & & & \\
\hline 128 & NR & & & & & & \\
\hline 141 & NR & & & $<3$ & & & \\
\hline 142 & 2 & 1.09 & & 1.50 & & & \\
\hline 146 & NR & & & & $<50$ & & \\
\hline 149 & NR & & & $<3$ & & & \\
\hline 179 & NR & & & $<5$ & & & \\
\hline 180 & NR & & & & $<27.1$ & & \\
\hline 194 & NR & & & $<5$ & & & \\
\hline 196 & 4 & -0.37 & & & & & \\
\hline 198 & NR & & & $<0.01$ & & & \\
\hline 210 & NR & & & $<5$ & & & \\
\hline 211 & NR & & & $<2$ & & & \\
\hline 217 & NR & & & & & & \\
\hline
\end{tabular}


Table 10. Statistical summary of reported data for standard reference water sample $T-129$ (trace constituents)-Continued Se (Selenium) $\mu \mathrm{g} / \mathrm{L}$
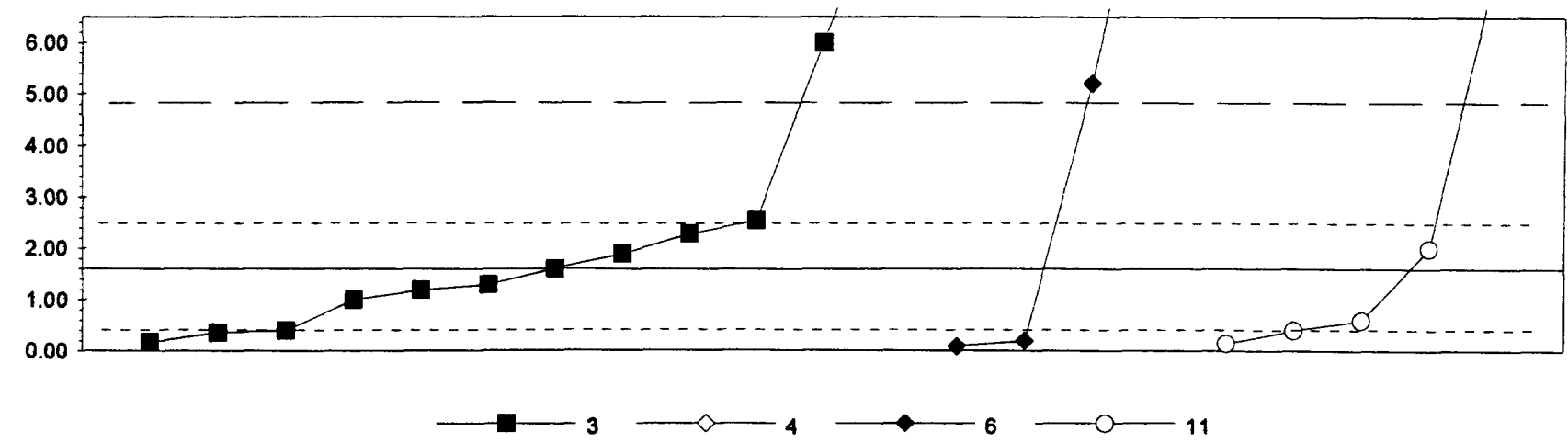

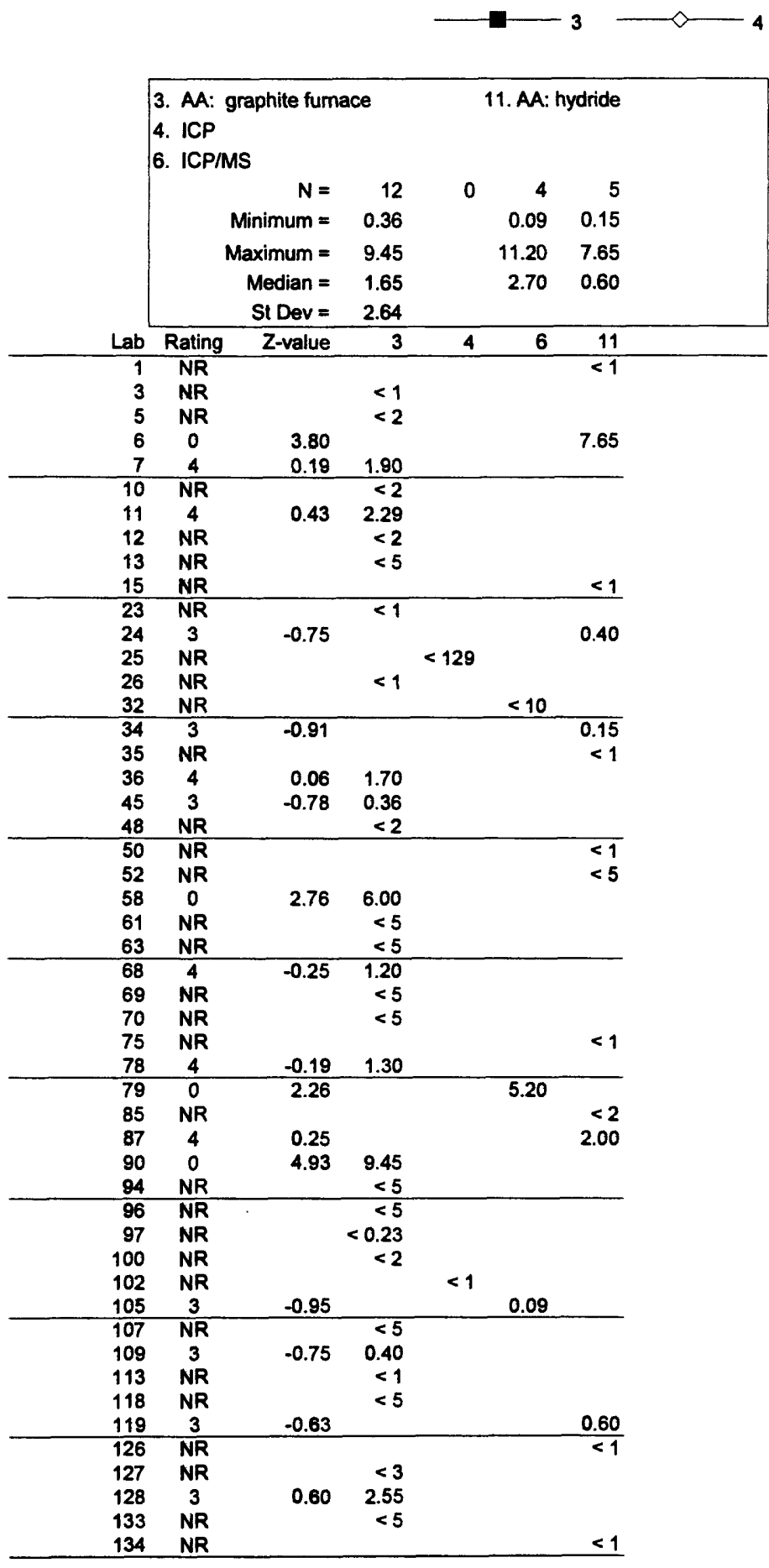

3. AA: graphite furnace

4. ICP

$11.20 \quad 7.65$
MPV $=\quad 1.60$

F-pseudosigma $=\quad 1.59$

$N=21$

$\mathrm{Hu}=2.55$

$H I=0.40$

\begin{tabular}{cccrrrr} 
Lab & Rating & Z-value & 3 & 4 & 6 & 11 \\
\hline 136 & NR & & $<10$ & & & $<2$ \\
141 & NR & & & & & \\
142 & 4 & 0.00 & 1.60 & & & \\
146 & NR & & $<10$ & & & \\
149 & NR & & $<2$ & & & \\
\hline 151 & NR & & & & & \\
179 & NR & & $<5$ & & & \\
180 & NR & & & $<45$ & \\
191 & 0 & 6.02 & & & 11.20 & \\
193 & NR & & $<5$ & & & \\
\hline 194 & NR & & $<5$ & & \\
196 & 3 & -0.88 & & & 0.20 & \\
198 & NR & & $<0.01$ & & \\
203 & NR & & $<5$ & & \\
204 & NR & & $<5$ & & \\
\hline 210 & NR & & $<5$ & & \\
211 & NR & & $<2$ & & \\
217 & NR & & & \\
220 & 4 & -0.38 & 1.00 & & \\
221 & NR & & $<1$ & \\
\hline
\end{tabular}


Table 10. -Statistical summary of reported data for standard reference water sample T-129 (trace constituents)--Continued $\mathrm{SiO} 2$ (Silica) $\mathrm{m} \mathbf{g} / \mathbf{L}$

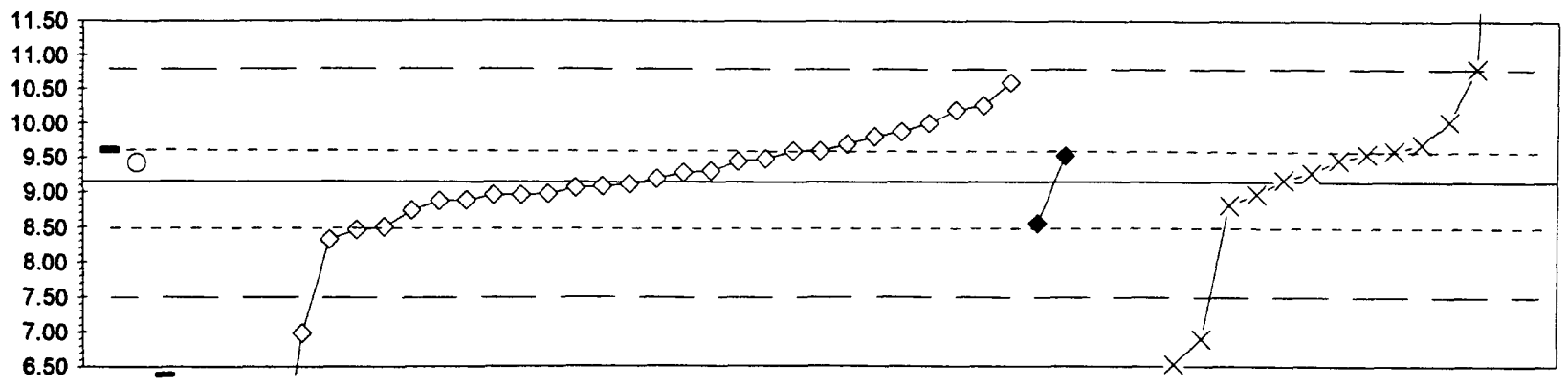

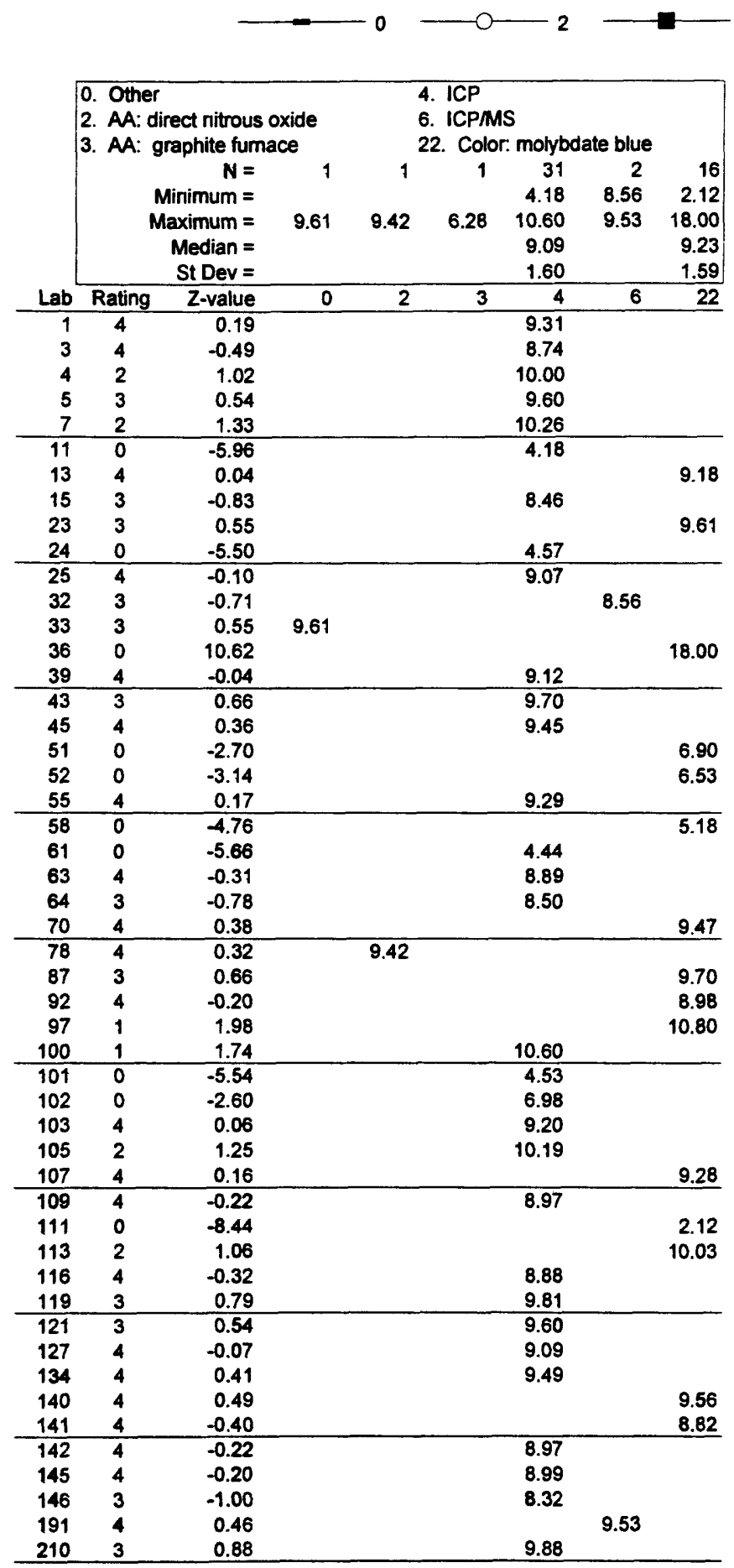
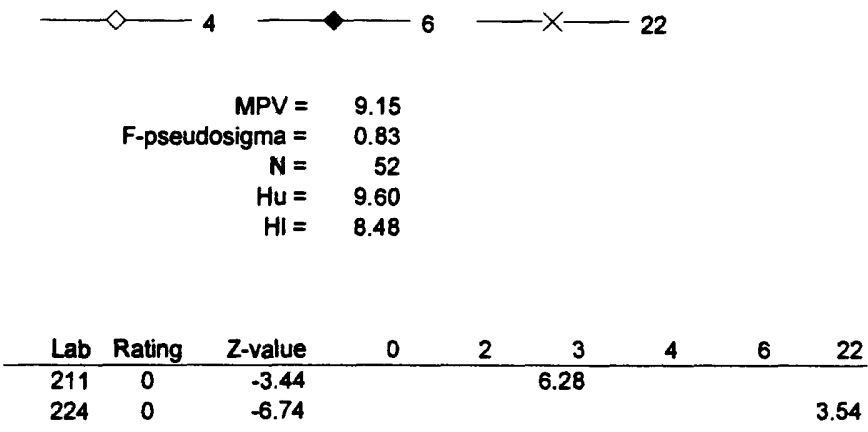

\begin{tabular}{rcrrrrrrr} 
Lab & Rating & Z-value & 0 & 2 & 3 & 4 & 6 & 22 \\
\hline 211 & 0 & -3.44 & & & 6.28 & & & \\
224 & 0 & -6.74 & & & & & & 3.54
\end{tabular}


Table 10. -Statistical summary of reported data for standard reference water sample T-129 (trace constituents)-Continued Sr (Strontium) $\mu \mathrm{g} / \mathrm{L}$

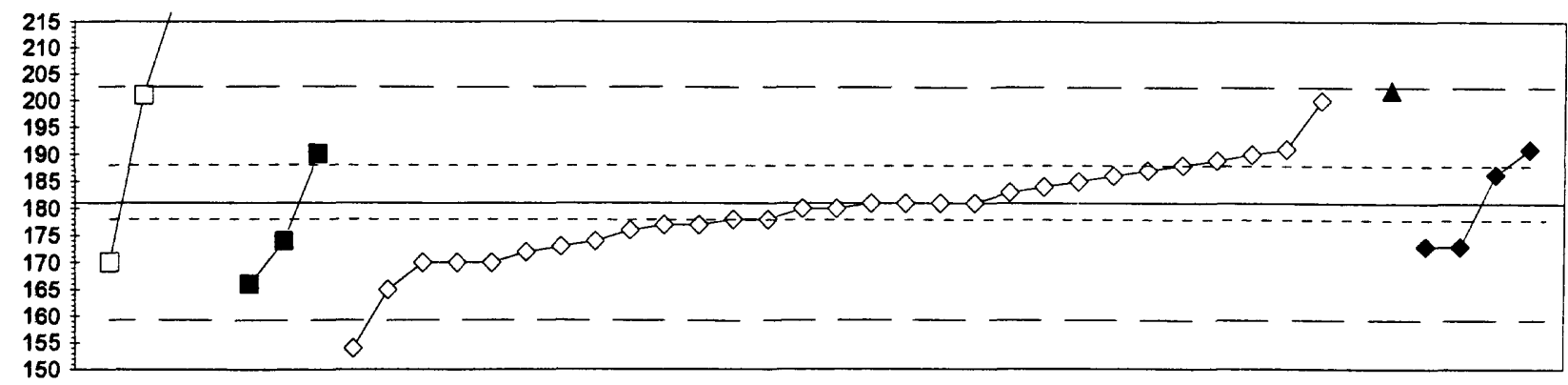

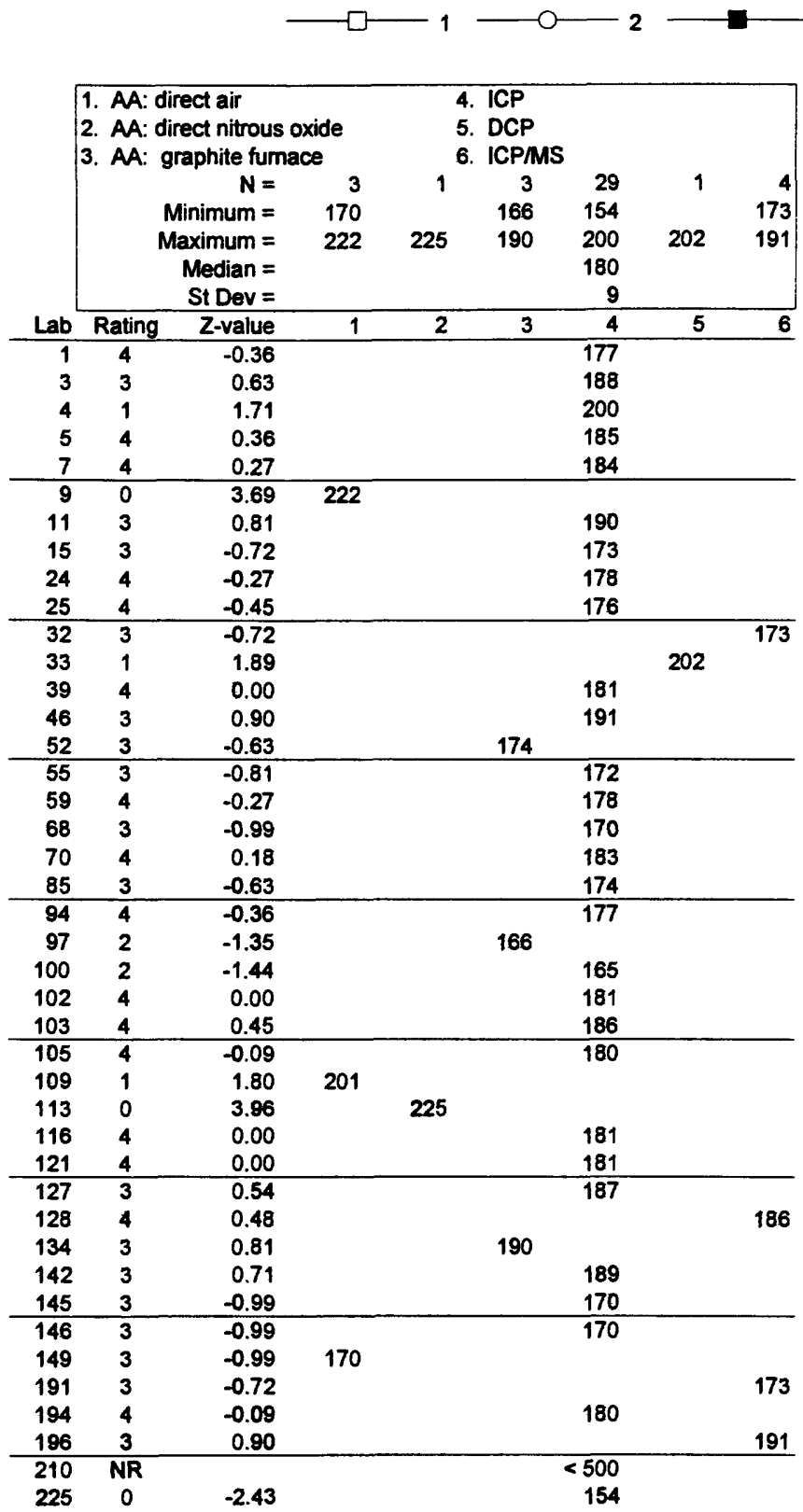


Table 10. -Statistical summary of reported data for standard reference water sample T-129 (trace constituents)--Continued V (Vanadium) $\mu \mathrm{g} / \mathrm{L}$

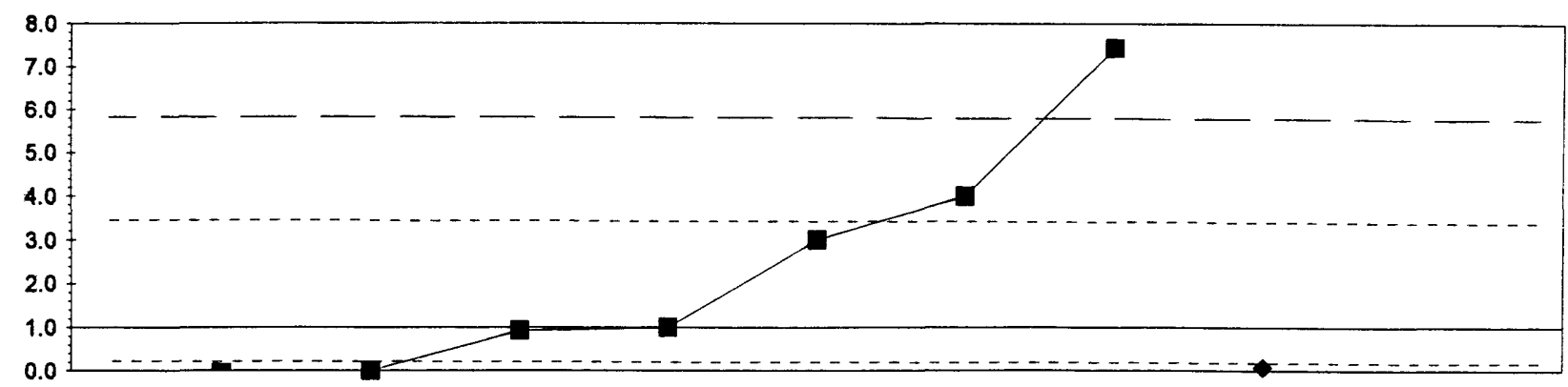

$\longrightarrow 0$

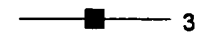

6. ICP/MS

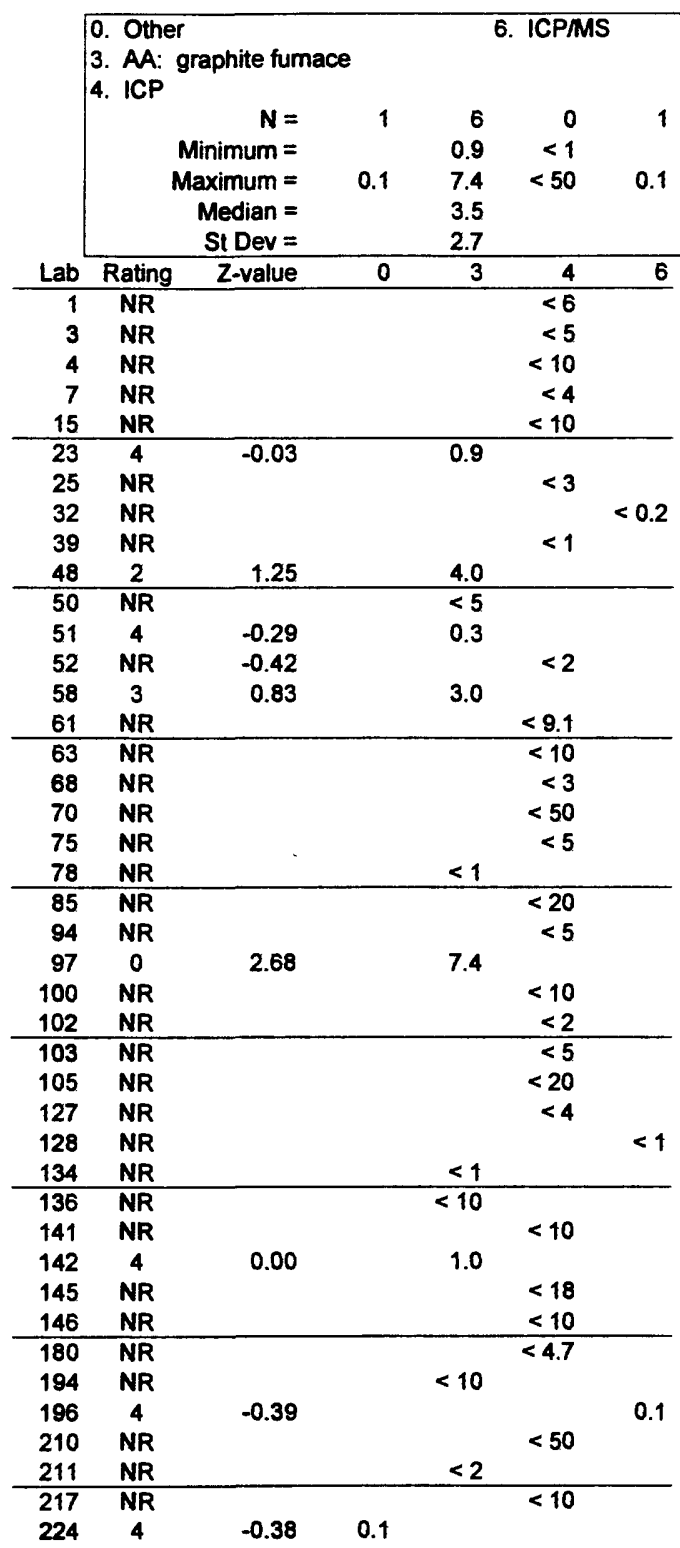


Table 10. -Statistical summary of reported data for standard reference water sample $T$-129 (trace constituents)-Continued Zn (Zinc) $\mu \quad g / L$

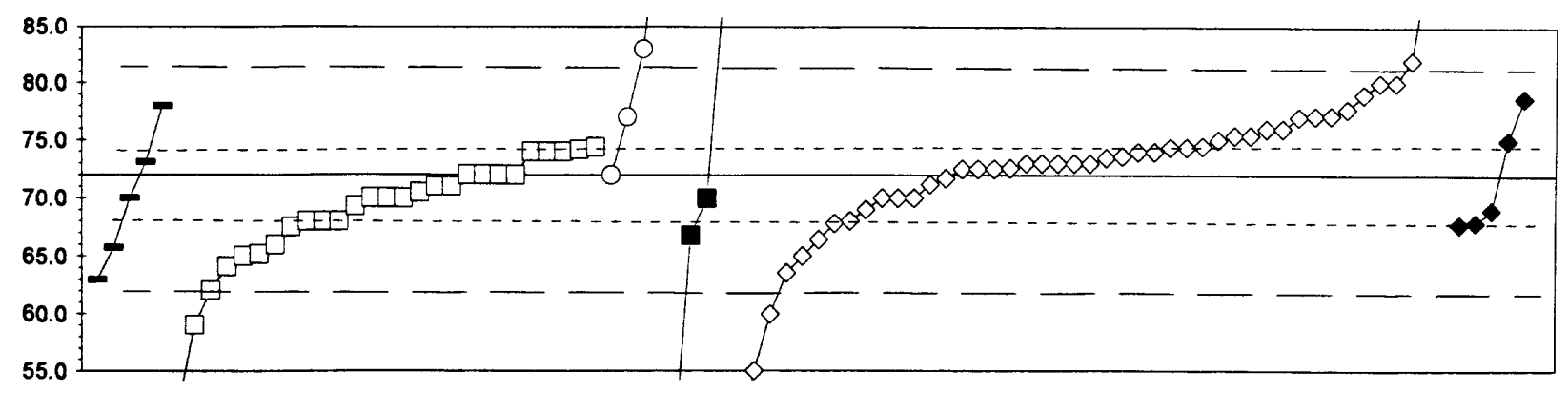

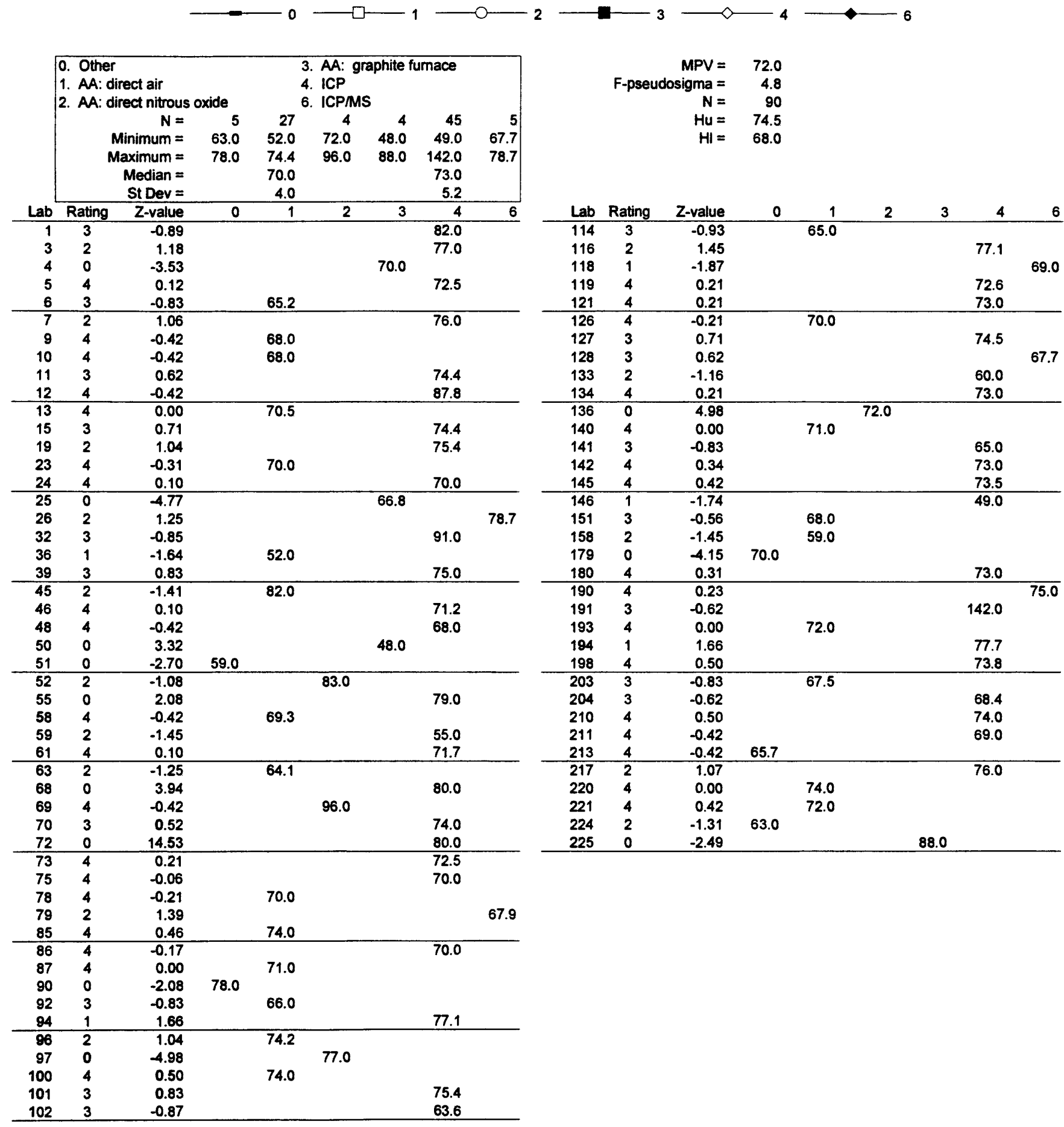


Table 11. -Statistical summary of reported data for standard reference water sample $M-130$ (major constituent

Definition of analytical methods, abbreviations, and symbols.

Analytical methods

0. Other/Not reported

1. AA: direct air

atomic absorption: direct, air

2. AA: direct $\mathrm{N} 2 \mathrm{O}$

atomic absorption: direct, nitrous oxide

3. AA: graphite furnace

atomic absorption: graphite furnace

4. ICP

inductively coupled plasma

5. DCP

direct current plasma

6. ICPMS

inductively coupled plasma/mass spectrometry

7. IC

ion chromatography

12. Flame emission

20. Titrate: color

titration: colorimetric [color reagent specified]

21. Titrate: electro

titration: electrometric

22: Color

colorimetric [color reagent specified]

40. Ion selective electrode

41. Electro

electrometric [direct reading instrument]

50. Gravimetric

gravimetric [precipitate specified]

51. Turbidimetric

Abbreviations and symbols

$$
\begin{aligned}
N & =\text { number of samples } \\
\text { Stdev } & =\text { traditional standard deviation } \\
M P V & =\text { most probable value }
\end{aligned}
$$

F-pseudosigma $=$ nonparametric statistic deviation

$\mathrm{Hu}=$ upper hinge value

$\mathrm{HI}=$ lower hinge value

$\mu g / L=$ micrograms per liter

$\mathrm{m} g / \mathrm{L}=$ milligrams par liter

$\mu \mathrm{cm}=$ microsiemens per centimeter at 25 degrees $C$

$\mathrm{Lab}=$ Laboratory code number

$N R=$ not rated, less than value reported

$<=$ less than

\begin{tabular}{llr}
\hline Constituent & & page \\
Alk & Acidity as CaCO3 & 60 \\
B & Boron & 61 \\
Ca & Calcium & 62 \\
CI & Chloride & 63 \\
DSRD & Dissolved solids & 64 \\
F & Fluoride & 65 \\
K & Potassium & 66 \\
Mg & Magnesium & 67 \\
Na & Sodium & 68 \\
total P & Phosphorus & 69 \\
PH & & 70 \\
SiO2 & Silica & 71 \\
SO4 & Sulfate & 72 \\
SpCond & Specific Conductance & 73 \\
Sr & Strontium & 74 \\
V & Vanadium & 75 \\
\hline
\end{tabular}


Table 11. -Statistical summary of reported data for standard reference water sample $M-130$ (major constituents)--Continued Alk (Alkalinity as calcium carbonate) $\mathrm{m} \mathbf{g} / \mathrm{L}$

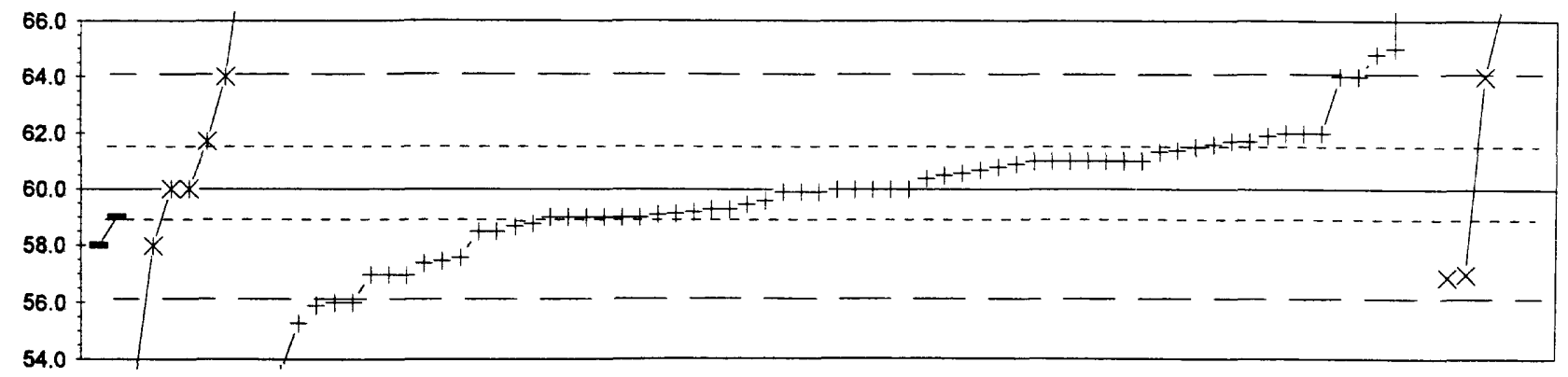

$\longrightarrow-0-20-21-x-22$

\begin{tabular}{|c|c|c|c|c|c|c|}
\hline \multirow[t]{2}{*}{ Lab } & \multicolumn{2}{|c|}{$\begin{array}{l}\text { 0. Other } \\
\text { 20. Titrate: colorimetric } \\
\text { 21. Titrate: electrometr } \\
\mathrm{N}= \\
\text { Minimum }= \\
\text { Maximum }= \\
\text { Median }= \\
\text { St Dev }=\end{array}$} & $\begin{array}{r}\text { ric } \\
2 \\
58.0 \\
59.0 \\
\end{array}$ & $\begin{array}{r}8 \\
53.3 \\
71.1 \\
60.9 \\
5.8\end{array}$ & $\begin{array}{r}65 \\
53.5 \\
285.0 \\
60.0 \\
2.2\end{array}$ & $\begin{array}{r}6 \\
56.9 \\
480.0 \\
\end{array}$ \\
\hline & Rating & Z-value & 0 & 20 & 21 & 22 \\
\hline 1 & 4 & -0.05 & & & 59.9 & \\
\hline 3 & 2 & 1.04 & & & 62.0 & \\
\hline 5 & 0 & 32.17 & & & 122.0 & \\
\hline 6 & 0 & 2.08 & & 64.0 & & \\
\hline 9 & 4 & 0.00 & & & 60.0 & \\
\hline 10 & 4 & 0.26 & & & 60.5 & \\
\hline 11 & 0 & 2.49 & & & 64.8 & \\
\hline 12 & 0 & 2.59 & & & 65.0 & \\
\hline 13 & 2 & -1.25 & & & 57.6 & \\
\hline 15 & 1 & -1.56 & & & 57.0 & \\
\hline 19 & 3 & 0.88 & & & 61.7 & \\
\hline 23 & 4 & -0.42 & & & 59.2 & \\
\hline 24 & 3 & -0.52 & & & 59.0 & \\
\hline 25 & 0 & 116.74 & & & 285.0 & \\
\hline 26 & 3 & -0.52 & 59.0 & & & \\
\hline 32 & 4 & 0.47 & & & 60.9 & \\
\hline 33 & 4 & -0.45 & & & 59.1 & \\
\hline 36 & 3 & -0.52 & & & 59.0 & \\
\hline 38 & 4 & 0.30 & & & 60.6 & \\
\hline 40 & 0 & -2.07 & & & 56.0 & \\
\hline 43 & 3 & -0.52 & & & 59.0 & \\
\hline 45 & 3 & -0.78 & & & 58.5 & \\
\hline 46 & 4 & -0.21 & & & 59.6 & \\
\hline 48 & 0 & 2.08 & & & & 64.0 \\
\hline 50 & 4 & 0.00 & & & 60.0 & \\
\hline 51 & 0 & 2.08 & & & 64.0 & \\
\hline 52 & 0 & 217.92 & & & & 480.0 \\
\hline 54 & 4 & 0.00 & & & 60.0 & \\
\hline 55 & 2 & 1.04 & & & 62.0 & \\
\hline 56 & 3 & 0.99 & & & 61.9 & \\
\hline 58 & 1 & -1.56 & & & 57.0 & \\
\hline 63 & 3 & -0.62 & & & 58.8 & \\
\hline 68 & 0 & 40.89 & & & & 139.0 \\
\hline 69 & 0 & 3.48 & & & & 66.7 \\
\hline 70 & 3 & -0.67 & & & 58.7 & \\
\hline 72 & 3 & 0.52 & & & 61.0 & \\
\hline 75 & 2 & -1.30 & & & 57.5 & \\
\hline 78 & 3 & -0.78 & & & 58.5 & \\
\hline 79 & 3 & 0.52 & & & 61.0 & \\
\hline 80 & 0 & 4.57 & & 68.8 & & \\
\hline 84 & 4 & 0.00 & & & 60.0 & \\
\hline 85 & 4 & -0.47 & & & 59.1 & \\
\hline 87 & 2 & 1.04 & & & 62.0 & \\
\hline 80 & 3 & 0.88 & & & 61.7 & \\
\hline 92 & 3 & 0.52 & & & 61.0 & \\
\hline 94 & 4 & 0.00 & & 60.0 & & \\
\hline 96 & 4 & -0.26 & & & 59.5 & \\
\hline 87 & 4 & -0.36 & & & 59.3 & \\
\hline 100 & 4 & -0.05 & & & 58.9 & \\
\hline 105 & 3 & 0.73 & & & 61.4 & \\
\hline
\end{tabular}

\begin{tabular}{|c|c|c|c|c|c|c|}
\hline Lab & Rating & Z-value & 0 & 20 & 21 & 22 \\
\hline 107 & 0 & -2.44 & & & 55.3 & \\
\hline 109 & 3 & 0.78 & & & 61.5 & \\
\hline 113 & 0 & -2.13 & & & 55.9 & \\
\hline 114 & 0 & 2.08 & & & 64.0 & \\
\hline 116 & 4 & -0.05 & & & 59.9 & \\
\hline 118 & 3 & 0.88 & & 61.7 & & \\
\hline 119 & 2 & -1.04 & & 58.0 & & \\
\hline 122 & 3 & 0.83 & & & 61.6 & \\
\hline 127 & 4 & 0.36 & & & 60.7 & \\
\hline 128 & 1 & -1.61 & & & & 56.9 \\
\hline 129 & 3 & 0.52 & 61.0 & & & \\
\hline 133 & 1 & -1.56 & & & 57.0 & \\
\hline 134 & 3 & 0.69 & & & 61.3 & \\
\hline 136 & 4 & 0.00 & & & 60.0 & \\
\hline 138 & 0 & -3.37 & & & 53.5 & \\
\hline 141 & 2 & -1.35 & & & 57.4 & \\
\hline 142 & 3 & 0.52 & & & 61.0 & \\
\hline 145 & 1 & -1.56 & & & & 57.0 \\
\hline 146 & 3 & -0.52 & & & 59.0 & \\
\hline 151 & 3 & 0.52 & & & 61.0 & \\
\hline 153 & 4 & 0.21 & & & 60.4 & \\
\hline 158 & 3 & -0.52 & & & 59.0 & \\
\hline 180 & 3 & 0.52 & & & 61.0 & \\
\hline 190 & 4 & -0.36 & & & 59.3 & \\
\hline 191 & 0 & 5.76 & & 71.1 & & \\
\hline 203 & 0 & -3.49 & & 53.3 & & \\
\hline 204 & 0 & -2.08 & & & 56.0 & \\
\hline 210 & 3 & 0.52 & & & 61.0 & \\
\hline 211 & 4 & 0.42 & & & 60.8 & \\
\hline 213 & 3 & -0.52 & & & 59.0 & \\
\hline 224 & 4 & 0.00 & & 60.0 & & \\
\hline \multirow[t]{2}{*}{225} & 2 & -1.04 & 58.0 & & & \\
\hline & & & & & & \\
\hline
\end{tabular}


Table 11. -Statistical summary of reported data for standard reference water sample $M-130$ (major constituents)--Continued B (Boron) $\mu \mathrm{g} / \mathrm{L}$
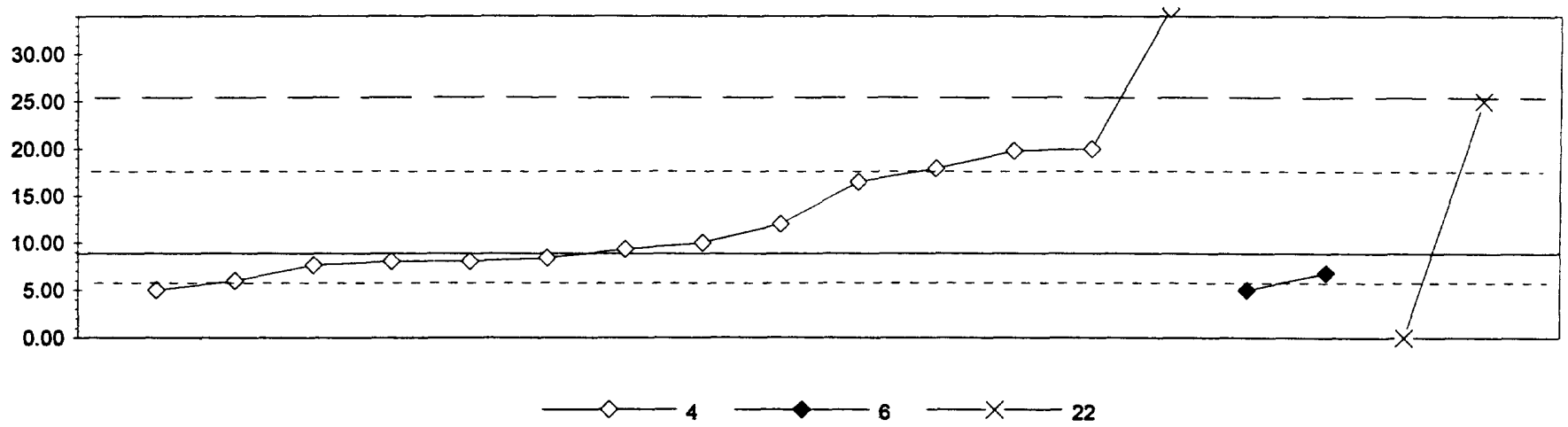

\begin{tabular}{|c|c|c|c|c|c|}
\hline \multirow[b]{2}{*}{ Lab } & \multicolumn{2}{|c|}{$\begin{aligned} \text { 4. ICP } \\
\text { 6. ICP/MS } \\
\text { 22. Colorimetric } \\
\mathrm{N}= \\
\text { Minimum }= \\
\text { Maximum }= \\
\text { Median }= \\
\text { St Dev }=\end{aligned}$} & $\begin{array}{r}14 \\
5.00 \\
34.80 \\
9.67 \\
8.05 \\
\end{array}$ & $\begin{array}{r}2 \\
5.00 \\
6.80\end{array}$ & $\begin{array}{r}2 \\
0.00 \\
25.00\end{array}$ \\
\hline & Rating & Z-value & 4 & 6 & 22 \\
\hline $\begin{array}{r}1 \\
3 \\
4 \\
10 \\
11 \\
\end{array}$ & $\begin{array}{c}4 \\
4 \\
\text { NR } \\
\text { NR } \\
4 \\
\end{array}$ & $\begin{array}{l}-0.15 \\
-0.06\end{array}$ & $\begin{array}{r}7.66 \\
8.40 \\
<5 \\
\\
5.00 \\
\end{array}$ & & $<50$ \\
\hline $\begin{array}{l}15 \\
25 \\
32 \\
36 \\
39\end{array}$ & $\begin{array}{c}2 \\
\text { NR } \\
4 \\
\text { NR } \\
\text { NR } \\
\end{array}$ & $\begin{array}{r}1.10 \\
-0.47 \\
-1.08\end{array}$ & $\begin{array}{r}17.90 \\
<23 \\
<10 \\
\end{array}$ & 5.00 & 0.00 \\
\hline $\begin{array}{l}46 \\
50 \\
52 \\
58 \\
63 \\
\end{array}$ & $\begin{array}{c}\text { NR } \\
\text { NR } \\
\text { NR } \\
1 \\
\text { NR } \\
\end{array}$ & 1.96 & $\begin{array}{r}<10 \\
<100 \\
<100 \\
\end{array}$ & & $\begin{array}{l}<100 \\
25.00\end{array}$ \\
\hline $\begin{array}{r}70 \\
85 \\
86 \\
94 \\
103 \\
\end{array}$ & $\begin{array}{c}\text { NR } \\
4 \\
3 \\
\text { NR } \\
4 \\
\end{array}$ & $\begin{array}{r}0.38 \\
0.93 \\
-0.35\end{array}$ & $\begin{array}{r}<50 \\
12.00 \\
16.50 \\
<10 \\
6.00 \\
\end{array}$ & & \\
\hline $\begin{array}{l}109 \\
119 \\
121 \\
127 \\
126\end{array}$ & $\begin{array}{l}4 \\
4 \\
2 \\
4 \\
0 \\
\end{array}$ & $\begin{array}{r}-0.10 \\
0.14 \\
1.35 \\
0.06 \\
3.15 \\
\end{array}$ & $\begin{array}{r}8.05 \\
10.00 \\
20.00 \\
9.34 \\
34.80 \\
\end{array}$ & & \\
\hline $\begin{array}{l}134 \\
138 \\
141 \\
142 \\
145\end{array}$ & $\begin{array}{c}\text { NR } \\
4 \\
2 \\
4 \\
\text { NR }\end{array}$ & $\begin{array}{r}-0.25 \\
1.33 \\
-0.10\end{array}$ & $\begin{array}{r}<20 \\
19.80 \\
8.05 \\
<23\end{array}$ & 6.80 & \\
\hline $\begin{array}{l}146 \\
180 \\
194 \\
210 \\
211\end{array}$ & $\begin{array}{l}\text { NR } \\
\text { NR } \\
\text { NR } \\
\text { NR } \\
\text { NR }\end{array}$ & & $\begin{array}{r}<50 \\
<11.8 \\
<100 \\
<50 \\
<40\end{array}$ & & \\
\hline
\end{tabular}


Table 11. -Statistical summary of reported data for standard reference water sample M-130 (major constituents)--Continued Ca (Calcium) $\mathrm{m} \mathrm{g} / \mathrm{L}$

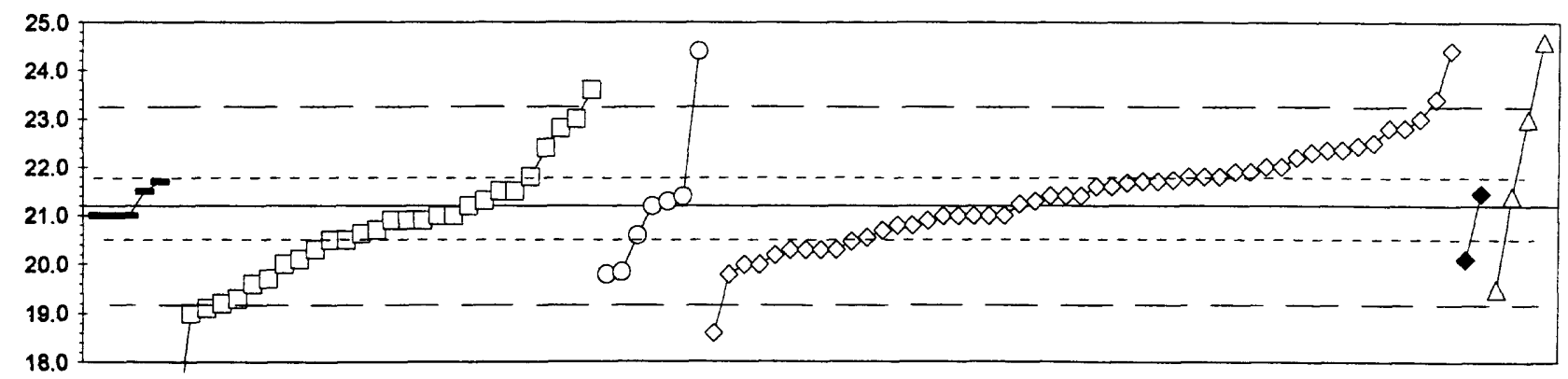

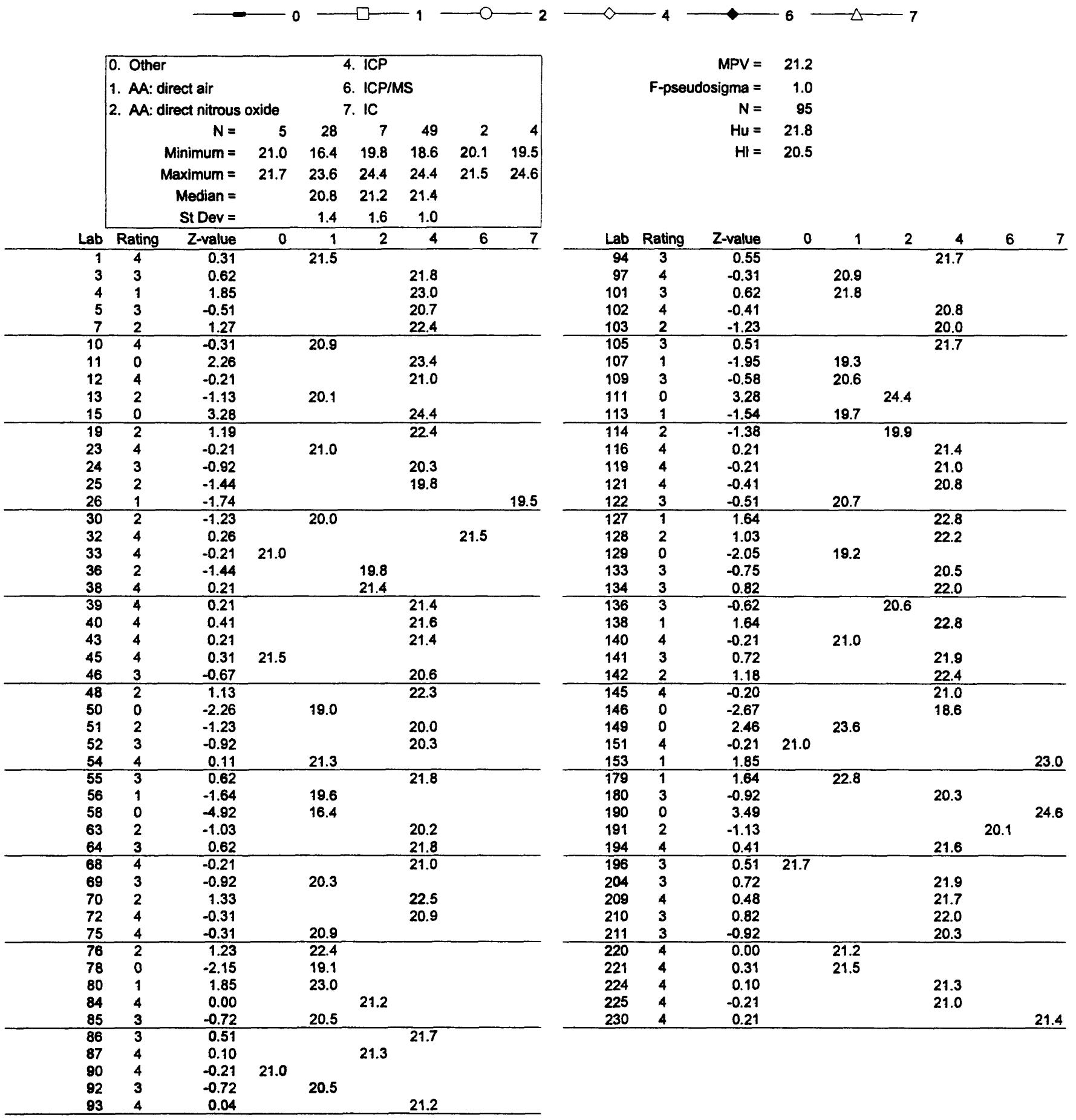


Table 11. -Statistical summary of reported data for standard reference water sample $M-130$ (major constituents)--Continued Cl (Chloride) $\mathrm{m} \mathbf{g} / \mathbf{L}$

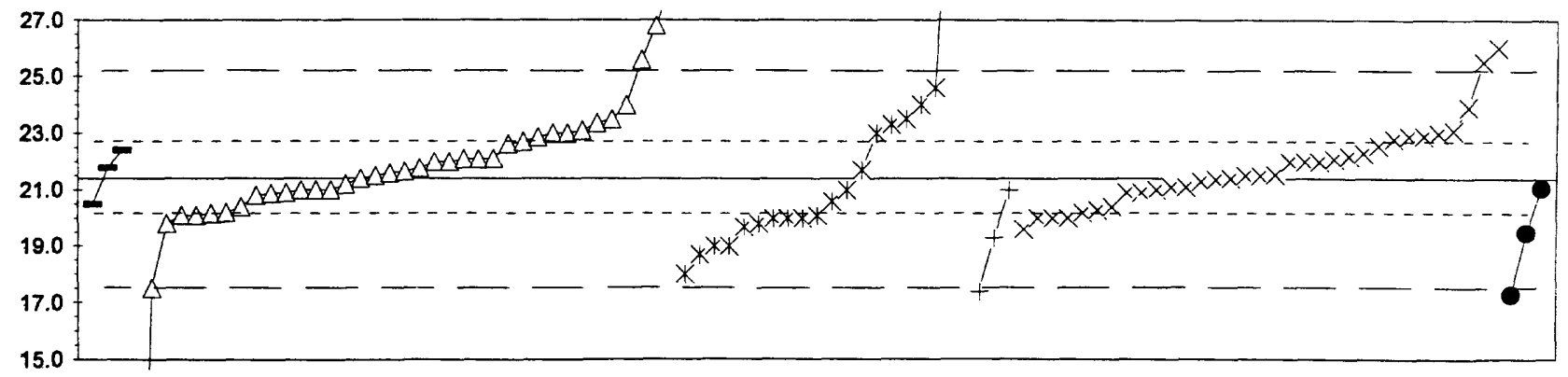

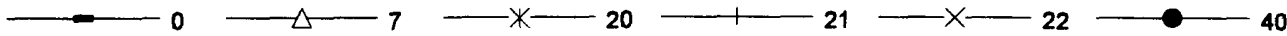

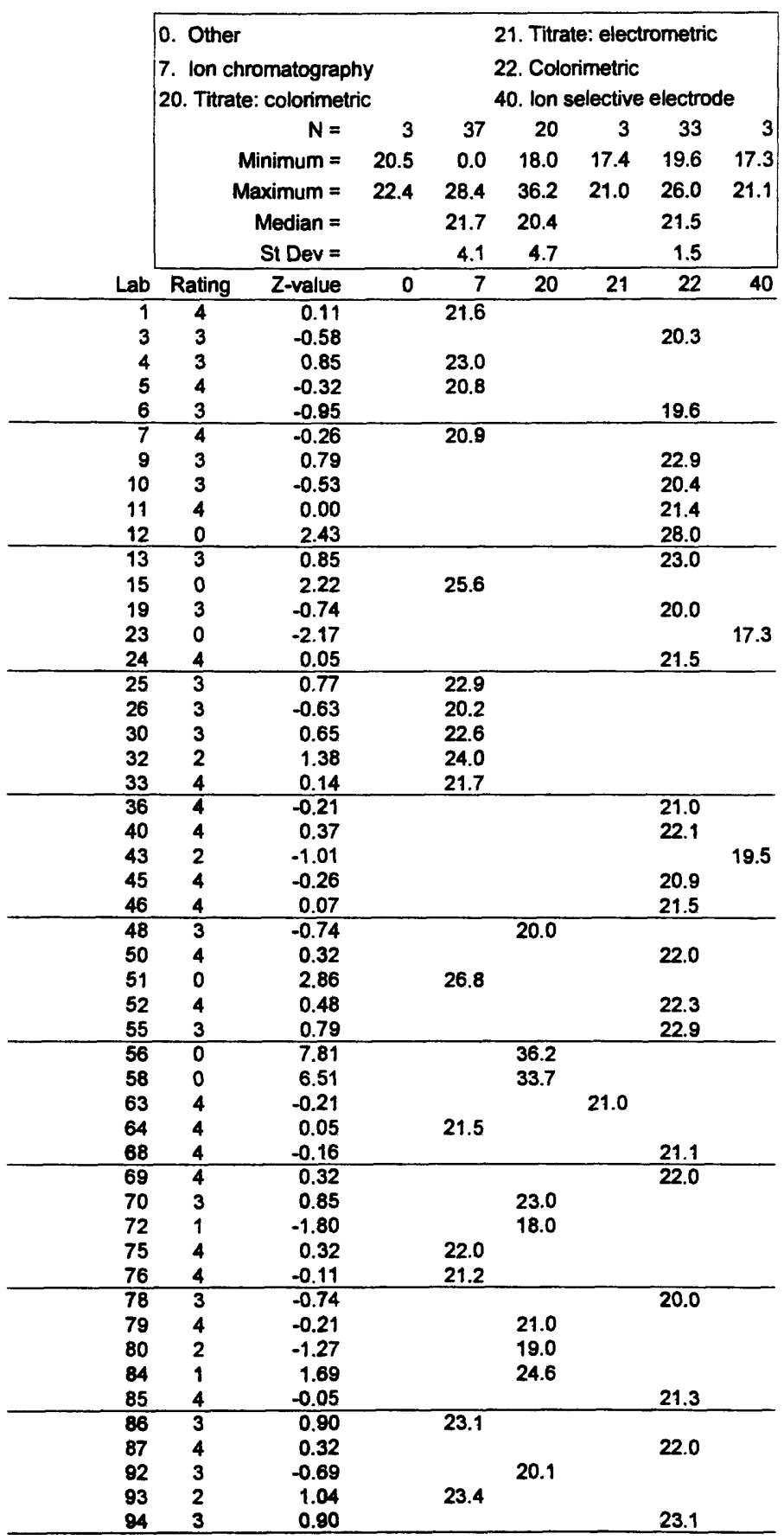

\begin{tabular}{|c|c|c|c|c|c|c|c|c|}
\hline Lab & Rating & Z-value & 0 & 7 & 20 & 21 & 22 & 40 \\
\hline 96 & 4 & 0.05 & & & & & 21.5 & \\
\hline 97 & 2 & 1.32 & & & & & 23.9 & \\
\hline 100 & 3 & -0.65 & & 20.2 & & & & \\
\hline 101 & 2 & -1.27 & & & 19.0 & & & \\
\hline 102 & 3 & -0.74 & & & & & 20.0 & \\
\hline 105 & 4 & -0.21 & & 21.0 & & & & \\
\hline 107 & 3 & -0.90 & & & 19.7 & & & \\
\hline 109 & 0 & -2.12 & & & & 17.4 & & \\
\hline 111 & 0 & 3.70 & & 28.4 & & & & \\
\hline 113 & 3 & -0.53 & & 20.4 & & & & \\
\hline 114 & 4 & -0.16 & & & & & & 21.1 \\
\hline 116 & 4 & -0.21 & & 21.0 & & & & \\
\hline 119 & 3 & -0.74 & & & 20.0 & & & \\
\hline 122 & 2 & -1.43 & & & 18.7 & & & \\
\hline 127 & 4 & -0.21 & & 21.0 & & & & \\
\hline 128 & 4 & -0.02 & & & & & 21.4 & \\
\hline 129 & 3 & 0.69 & & 22.7 & & & & \\
\hline 131 & 3 & -0.69 & & 20.1 & & & & \\
\hline 134 & 4 & 0.20 & & 21.8 & & & & \\
\hline 136 & 3 & 0.85 & & 23.0 & & & & \\
\hline 138 & 4 & 0.00 & & 21.4 & & & & \\
\hline 140 & 4 & 0.42 & & & & & 22.2 & \\
\hline 141 & 4 & -0.26 & & & & & 20.9 & \\
\hline 142 & 3 & 0.74 & & & & & 22.8 & \\
\hline 143 & 3 & 0.62 & & & & & 22.6 & \\
\hline 145 & 2 & 1.11 & & 23.5 & & & & \\
\hline 146 & 0 & 2.17 & & & & & 25.5 & \\
\hline 153 & 3 & -0.85 & & 19.8 & & & & \\
\hline 158 & 4 & -0.16 & & & & & 21.1 & \\
\hline 179 & 3 & -0.74 & & & 20.0 & & & \\
\hline 180 & 3 & -0.63 & & & & & 20.2 & \\
\hline 183 & 2 & 1.11 & & & 23.5 & & & \\
\hline 190 & 4 & 0.37 & & 22.1 & & & & \\
\hline 191 & 4 & 0.32 & & 22.0 & & & & \\
\hline 193 & 0 & -11.31 & & 0.0 & & & & \\
\hline 194 & 4 & -0.48 & 20.5 & & & & & \\
\hline 196 & 3 & 0.53 & 22.4 & & & & & \\
\hline 203 & 4 & -0.42 & & & 20.6 & & & \\
\hline 204 & 2 & -1.11 & & & & 19.3 & & \\
\hline 208 & 3 & -0.69 & & 20.1 & & & & \\
\hline 209 & 4 & -0.28 & & 20.9 & & & & \\
\hline 210 & 4 & 0.20 & 21.8 & & & & & \\
\hline 211 & 4 & 0.16 & & & 21.7 & & & \\
\hline 213 & 0 & -2.06 & & 17.5 & & & & \\
\hline 220 & 2 & 1.01 & & & 23.3 & & & \\
\hline 221 & 3 & -0.85 & & & 19.8 & & & \\
\hline 224 & 4 & 0.38 & & 22.1 & & & & \\
\hline 225 & 2 & 1.38 & & & 24.0 & & & \\
\hline 230 & 4 & 0.37 & & 22.1 & & & & \\
\hline
\end{tabular}


Table 11. -Statistical summary of reported data for standard reference water sample M-130 (major constituents)--Continued DSRD (Dissolved solids) $\mathrm{m}$ g/L

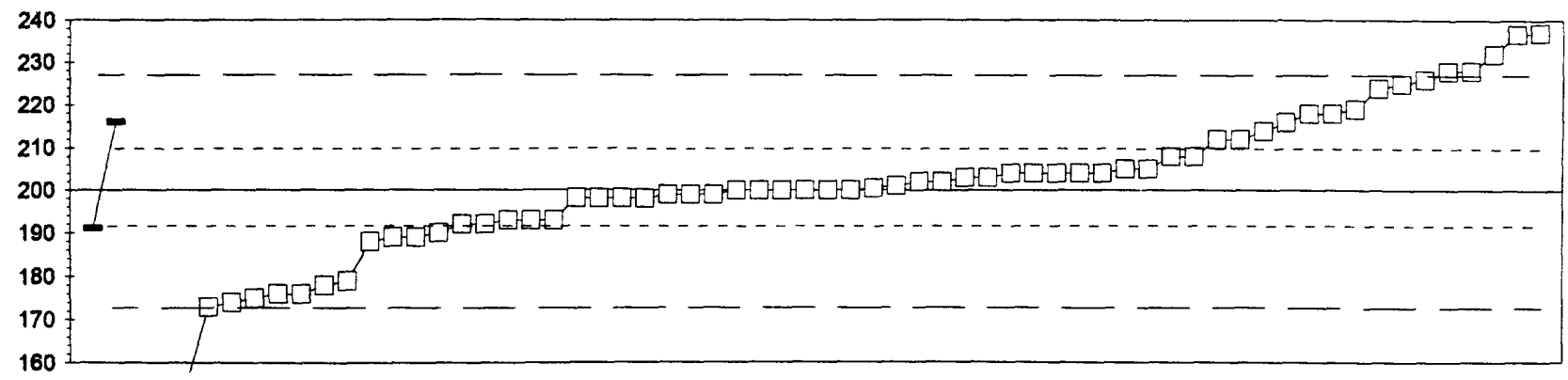

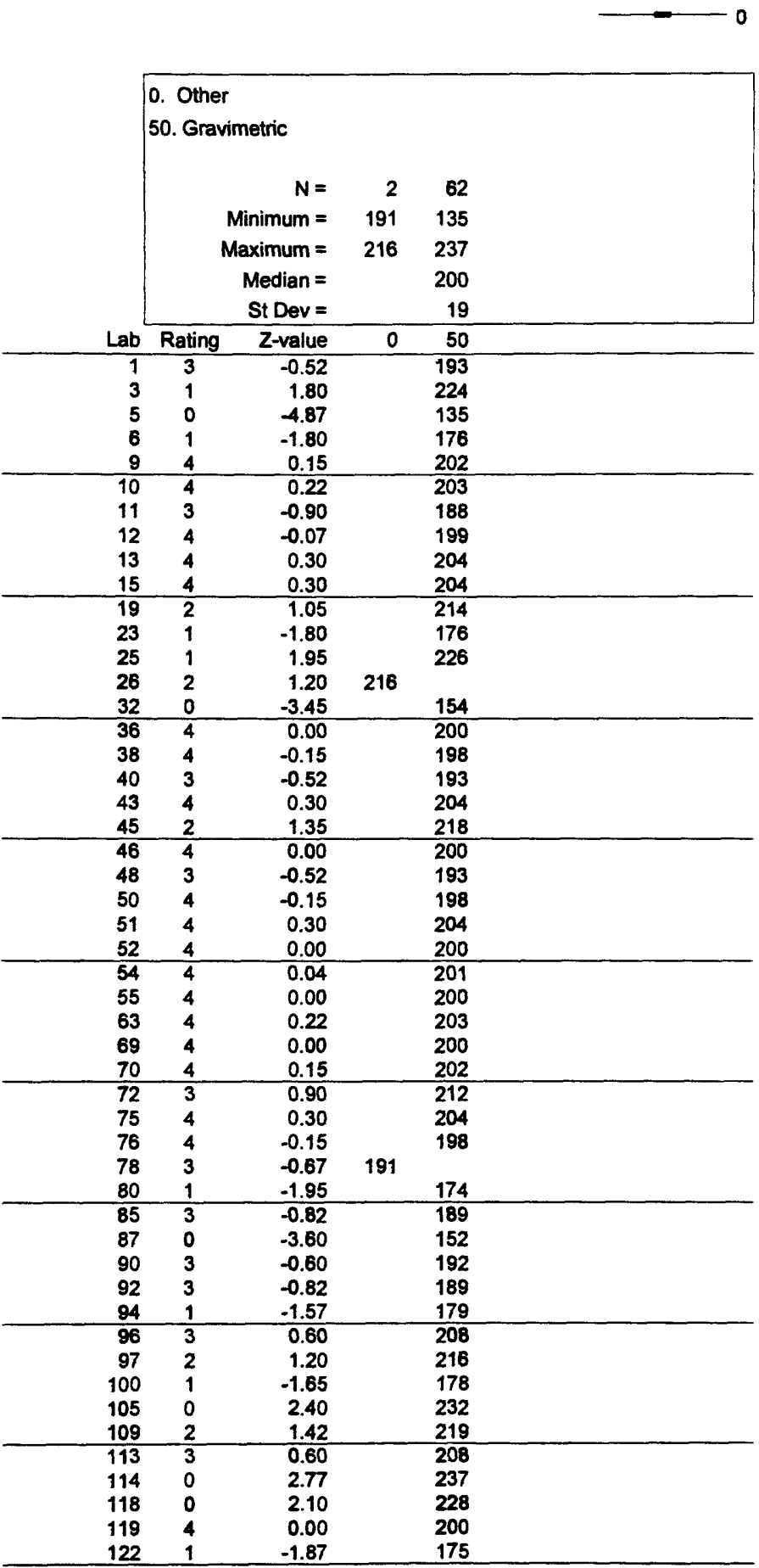

$\square-50$

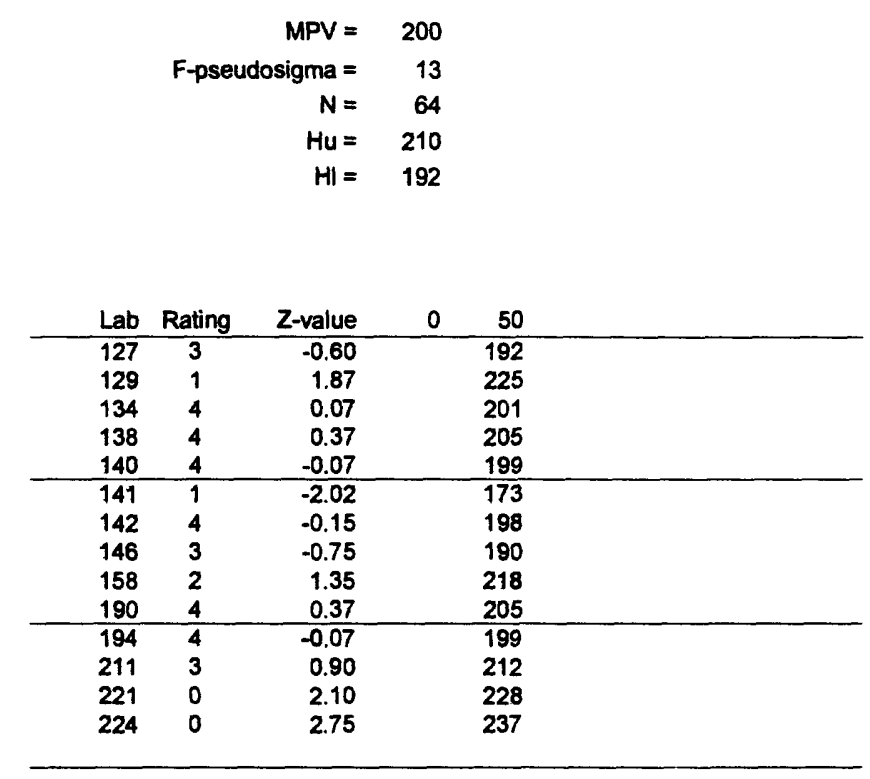


Table 11. -Statistical summary of reported data for standard reference water sample $M-130$ (major constituents)--Continued F (Fluoride) $\mathrm{m} \mathbf{g} / \mathbf{L}$

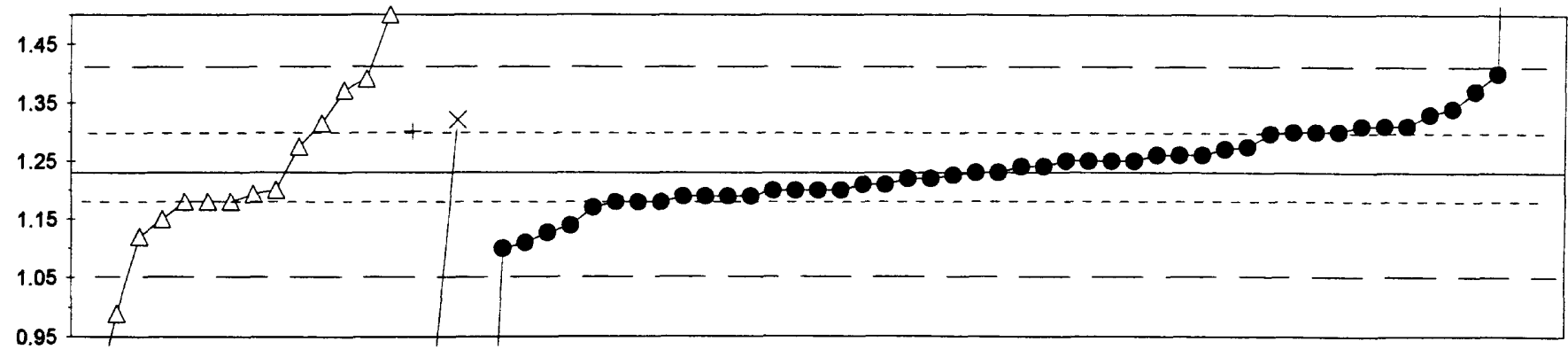

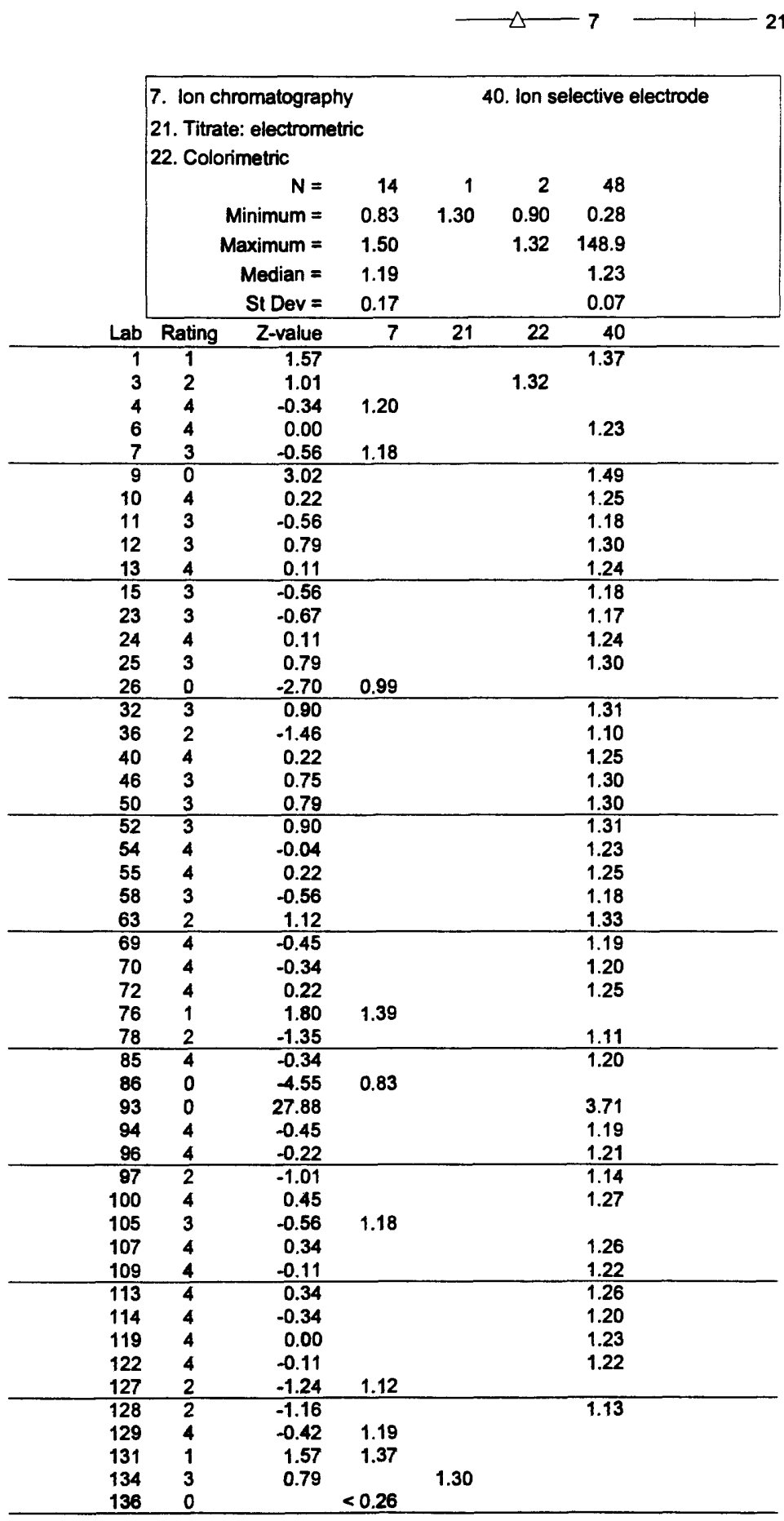

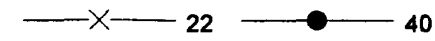

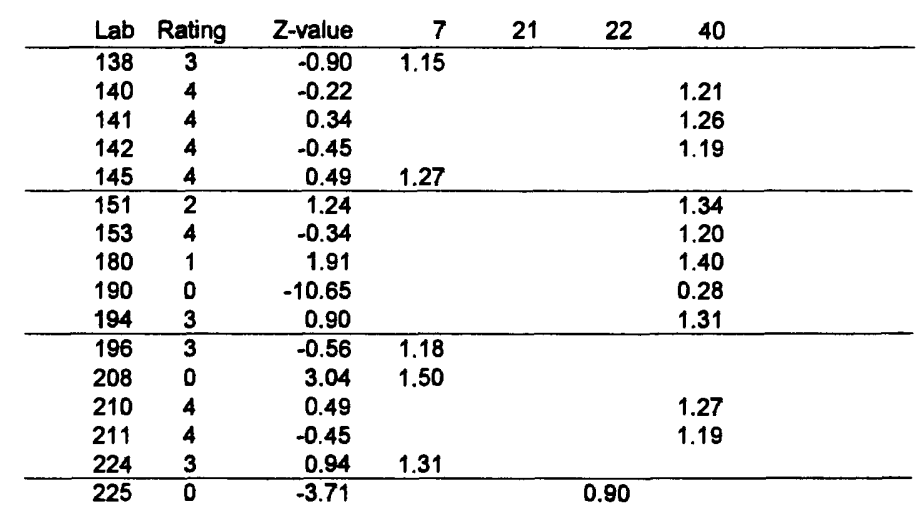


Table 11. -Statistical summary of reported data for standard reference water sample $M-130$ (major constituents)--Continued K (Potassium) $\mathrm{m} \mathrm{g} / \mathrm{L}$

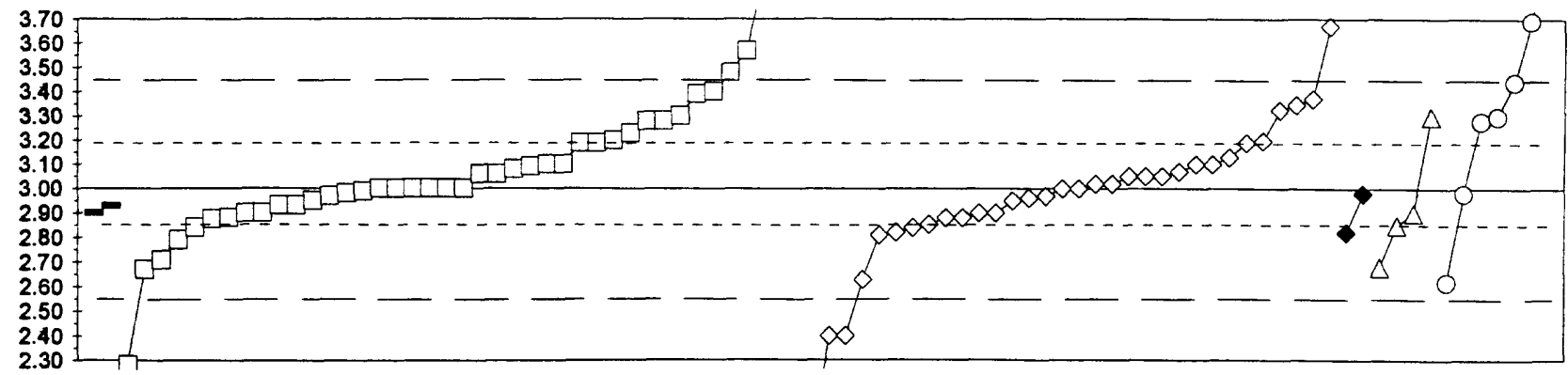

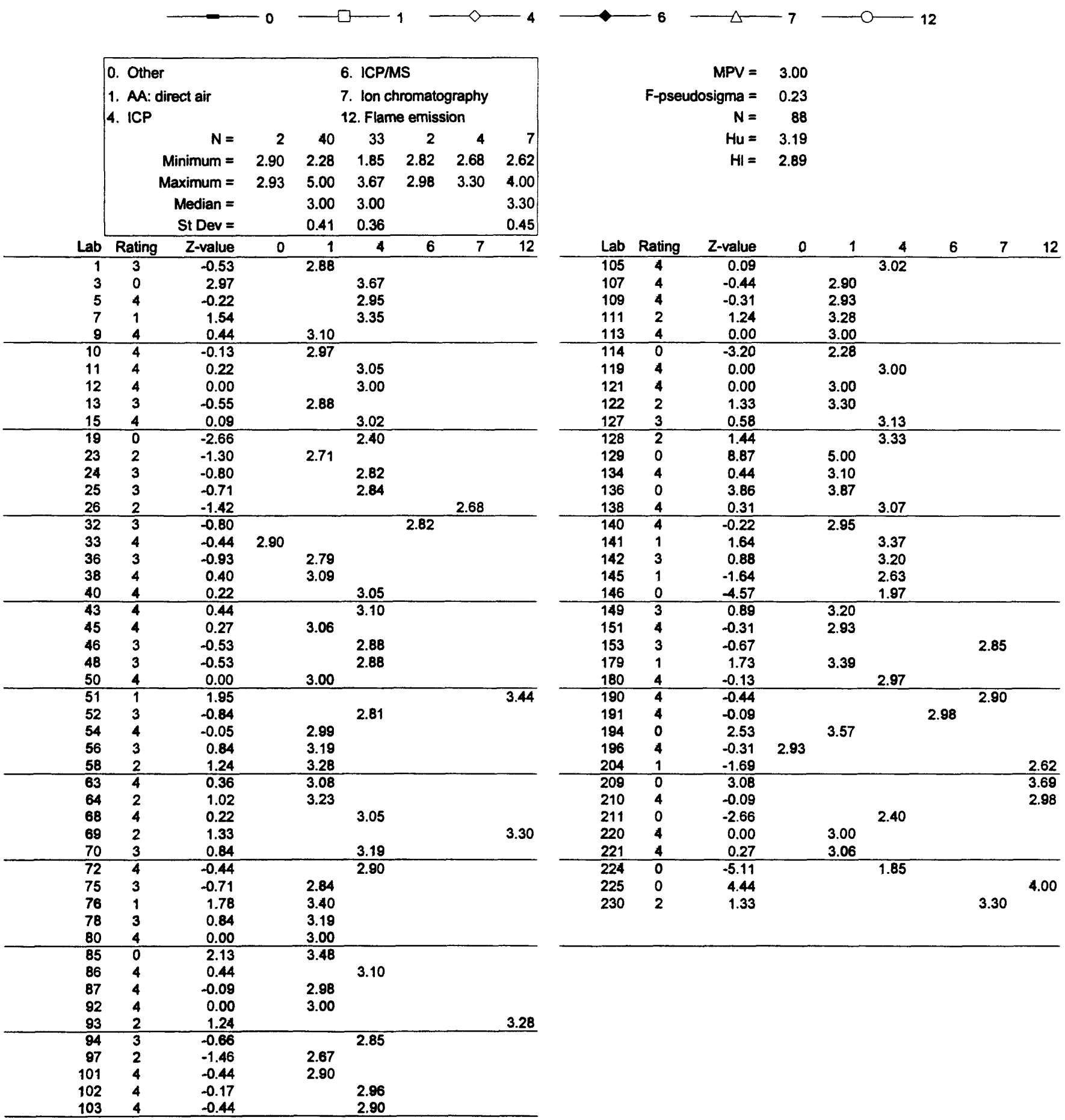


Table 11. -Statistical summary of reported data for standard reference water sample $M-130$ (major constituents)--Continued Mg (Magnesium) m g/L

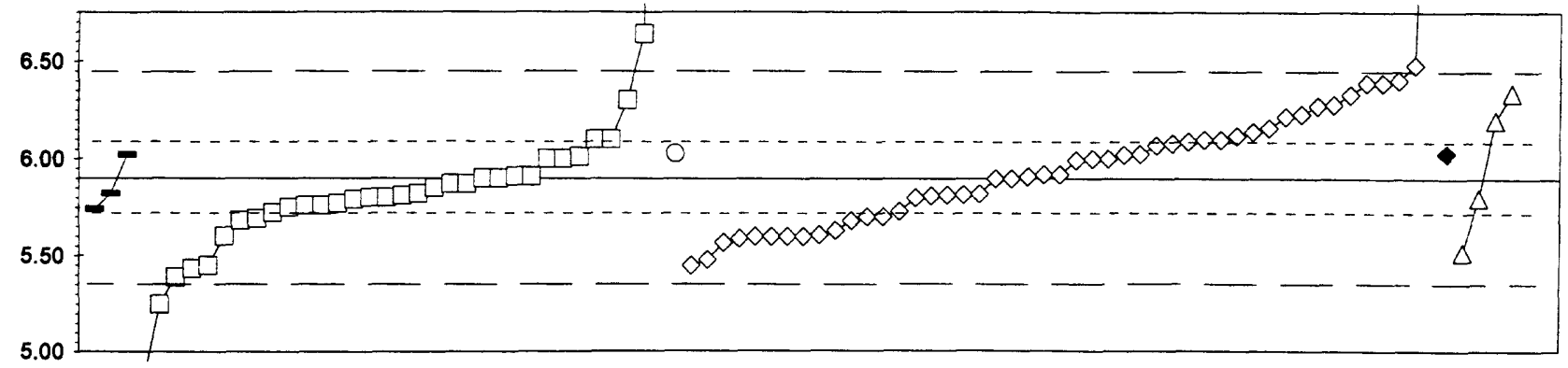

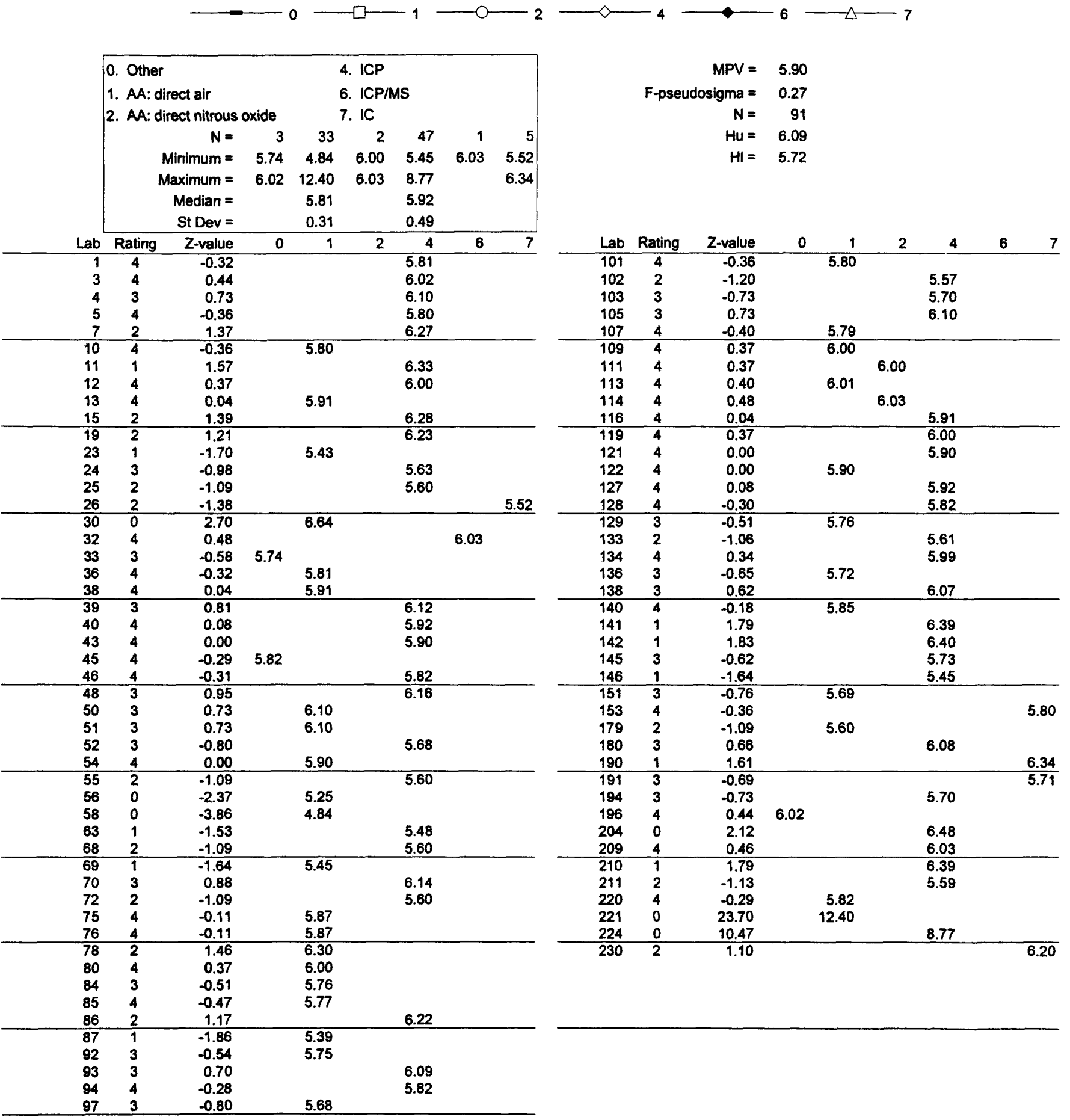


Table 11. -Statistical summary of reported data for standard reference water sample $M-130$ (major constituents)-Continued $\mathrm{Na}$ (Sodium) $\mathrm{m} \mathbf{g} / \mathbf{L}$

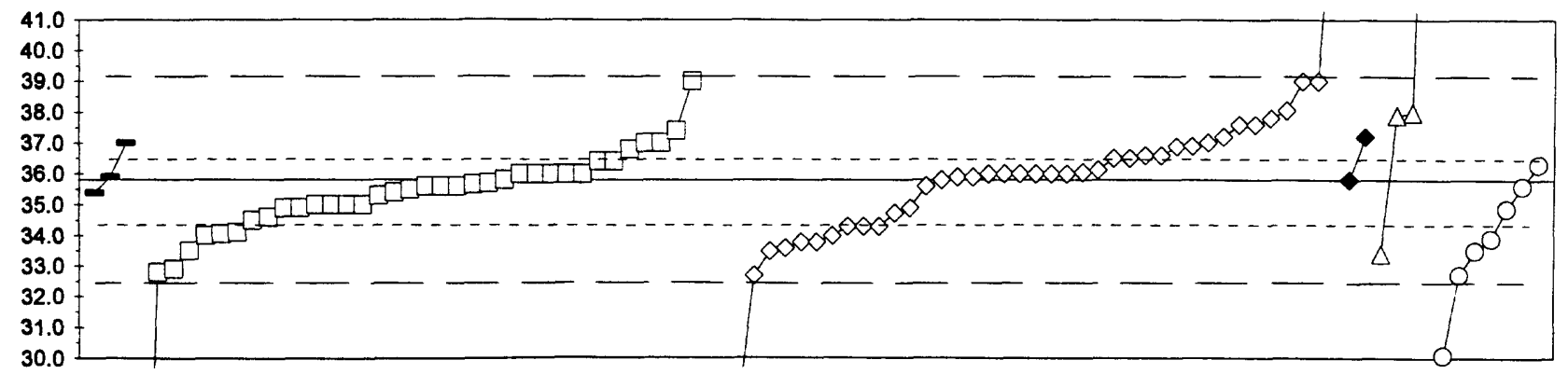

0. Other

1. AA: direct air

4. ICP
6. ICP/MS

7. Ion chromatography

12. Flame emission

$\begin{array}{lllllll}N= & 3 & 36 & 41 & 2 & 4 & 7\end{array}$

Minimum $=\quad \begin{array}{llllll}35.4 & 18.0 & 3.9 & 35.8 & 33.4 & 30.1\end{array}$

Maximum $=\quad \begin{array}{llllll}37.0 & 39.0 & 46.0 & 37.2 & 53.6 & 36.3\end{array}$

Median $=\quad 35.6 \quad 36.0 \quad 33.9$

St Dev = $\quad 3.2 \quad 2.0 \quad 2.1$

\begin{tabular}{rrrrrrrrr} 
Lab & Rating & Z-value & 0 & 1 & 4 & 6 & 7 & 12 \\
\hline 1 & 3 & -0.74 & & 34.6 & & & & \\
3 & 0 & -4.66 & & & 28.2 & & & \\
4 & 1 & 1.96 & & & 39.0 & & & \\
5 & 4 & 0.43 & & & 36.5 & & &
\end{tabular}

\begin{tabular}{rrrrr}
7 & 2 & 1.39 & & 38.1 \\
\hline 9 & 4 & 0.12 & 36.0 & \\
10 & 4 & -0.12 & 35.6 & \\
11 & 2 & 1.10 & 37.6 \\
12 & 4 & 0.12 & 36.0
\end{tabular}

\begin{tabular}{lllll}
13 & 4 & 0.00 & 35.8 & \\
\hline 15 & 1 & 1.96 & & 39.0 \\
19 & 3 & 0.66 & & 36.9 \\
23 & 2 & -1.04 & 34.1 & \\
24 & 2 & -1.41 & & 33.5
\end{tabular}

25

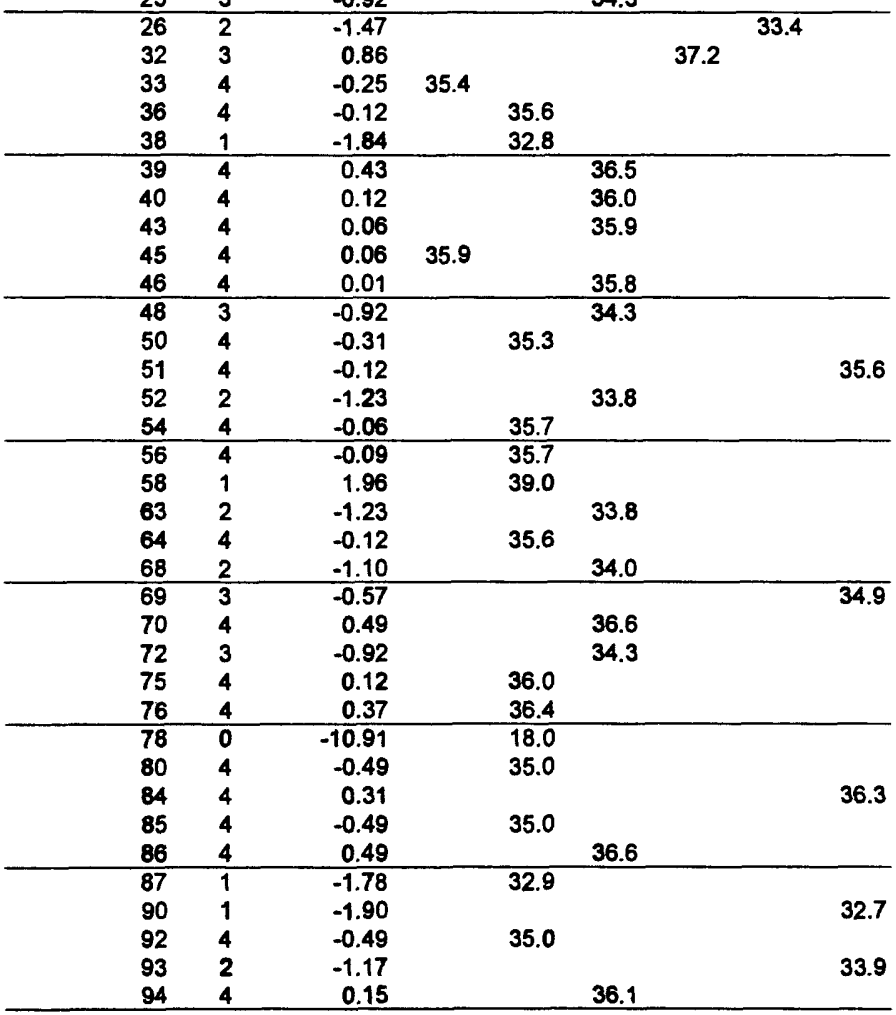

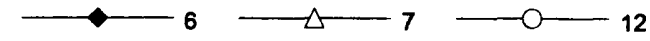

MPV $=\quad 35.8$

F-pseudosigma $=1.6$

$\mathbf{N}=93$

$\mathrm{Hu}=36.5$

$H I=34.3$

\begin{tabular}{|c|c|c|c|c|c|c|c|c|}
\hline Lab & Rating & Z-value & 0 & 1 & 4 & 6 & 7 & 12 \\
\hline 97 & 3 & -0.80 & & 34.5 & & & & \\
\hline 101 & 4 & 0.12 & & 36.0 & & & & \\
\hline 102 & 1 & -1.90 & & & 32.7 & & & \\
\hline 103 & 4 & 0.12 & & & 36.0 & & & \\
\hline 105 & 3 & 0.86 & & & 37.2 & & & \\
\hline 107 & 2 & -1.41 & & 33.5 & & & & \\
\hline 109 & 2 & -1.07 & & 34.1 & & & & \\
\hline 111 & 3 & -0.55 & & 34.9 & & & & \\
\hline 113 & 4 & 0.37 & & 36.4 & & & & \\
\hline 114 & 4 & -0.18 & & 35.5 & & & & \\
\hline 116 & 4 & 0.06 & & & 35.9 & & & \\
\hline 119 & 4 & 0.12 & & & 36.0 & & & \\
\hline 121 & 4 & 0.12 & & & 36.0 & & & \\
\hline 122 & 3 & 0.61 & & 36.8 & & & & \\
\hline 127 & 4 & -0.12 & & & 35.6 & & & \\
\hline 128 & 4 & 0.20 & & & 36.1 & & & \\
\hline 129 & 3 & 0.74 & & 37.0 & & & & \\
\hline 134 & 4 & 0.12 & & 36.0 & & & & \\
\hline 136 & 3 & 0.74 & & 37.0 & & & & \\
\hline 138 & 3 & 0.67 & & & 36.9 & & & \\
\hline 140 & 4 & 0.12 & & 36.0 & & & & \\
\hline 141 & 2 & 1.23 & & & 37.8 & & & \\
\hline 142 & 3 & 0.74 & & & 37.0 & & & \\
\hline 145 & 3 & -0.65 & & & 34.7 & & & \\
\hline 146 & 2 & -1.35 & & & 33.6 & & & \\
\hline 149 & 4 & -0.49 & & 35.0 & & & & \\
\hline 151 & 4 & -0.25 & & 35.4 & & & & \\
\hline 153 & 0 & 10.91 & & & & & 53.6 & \\
\hline 179 & 3 & 0.98 & & 37.4 & & & & \\
\hline 180 & 3 & -0.55 & & & 34.9 & & & \\
\hline 190 & 2 & 1.29 & & & & & 37.9 & \\
\hline 191 & 4 & 0.00 & & & & 35.8 & & \\
\hline 194 & 2 & 1.10 & & & 37.6 & & & \\
\hline 196 & 3 & 0.74 & 37.0 & & & & & \\
\hline 204 & 2 & -1.41 & & & & & & 33.5 \\
\hline 209 & 0 & -3.50 & & & & & & 30.1 \\
\hline 210 & 0 & -19.54 & & & 3.9 & & & \\
\hline 211 & 4 & 0.12 & & & 36.0 & & & \\
\hline 220 & 2 & -1.10 & & 34.0 & & & & \\
\hline 221 & 3 & -0.55 & & 34.9 & & & & \\
\hline 224 & 0 & -11.56 & & & 16.9 & & & \\
\hline 225 & 0 & 6.25 & & & 46.0 & & & \\
\hline 230 & 2 & 1.35 & & & & & 38.0 & \\
\hline
\end{tabular}


Table 11. -Statistical summary of reported data for standard reference water sample M-130 (major constituents)--Continued total P (total Phosohorus) $\quad \mathrm{m}$ g/L
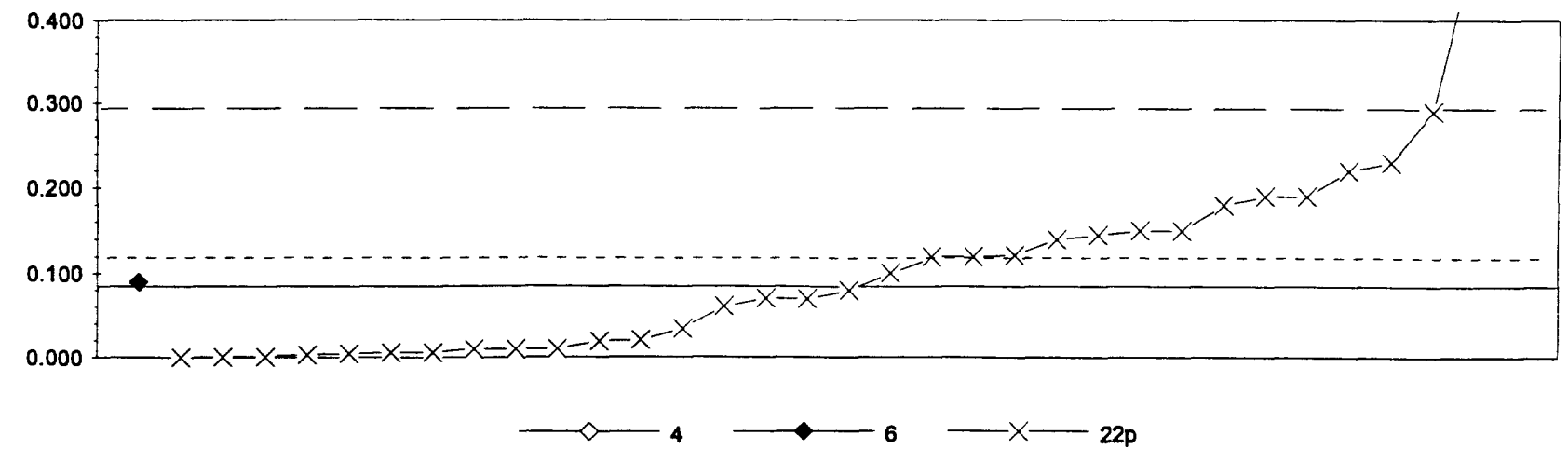

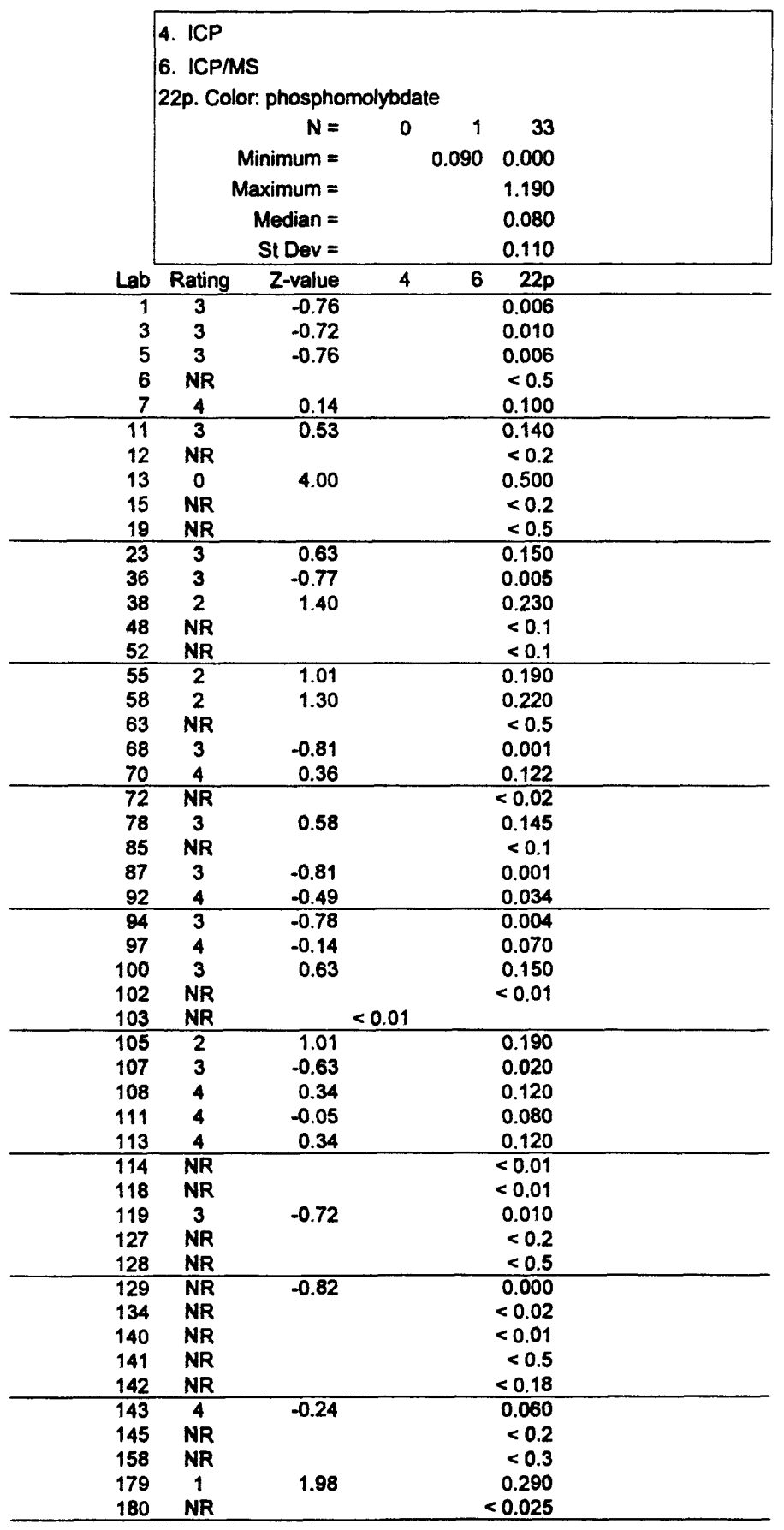

\begin{tabular}{|c|c|c|c|c|}
\hline Lab & Rating & Z-value & 46 & $22 p$ \\
\hline 191 & 4 & 0.05 & 0.090 & \\
\hline 194 & NR & & & $<0.1$ \\
\hline 203 & 3 & -0.64 & & 0.019 \\
\hline 204 & 4 & -0.14 & & 0.070 \\
\hline 210 & NR & & & $<0.1$ \\
\hline 211 & 0 & 10.65 & & 1.190 \\
\hline 213 & NR & & & $<0.2$ \\
\hline 221 & 3 & -0.72 & & 0.010 \\
\hline 224 & 3 & 0.92 & & 0.180 \\
\hline
\end{tabular}


Table 11. -Statistical summary of reported data for standard reference water sample $\mathbf{M - 1 3 0}$ (major constituents)--Continued pH

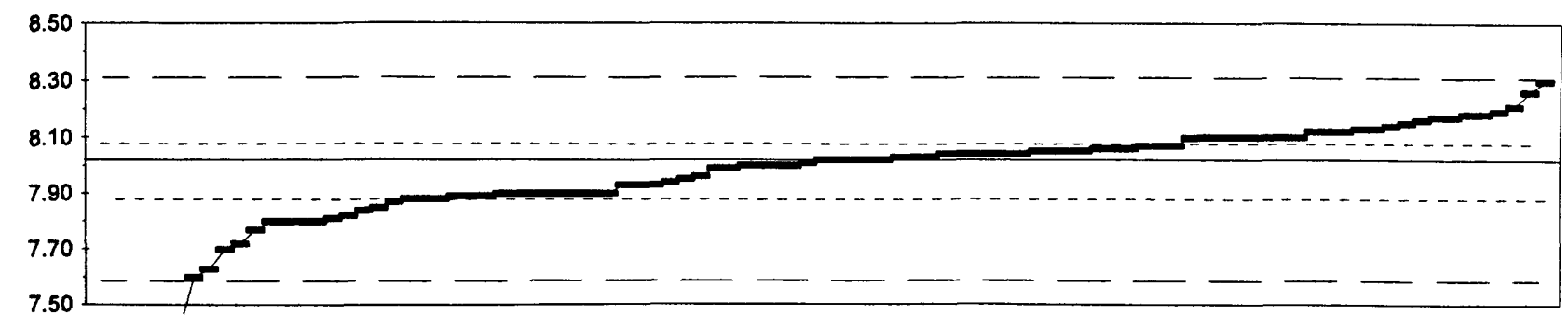

$\longrightarrow-\square 1$

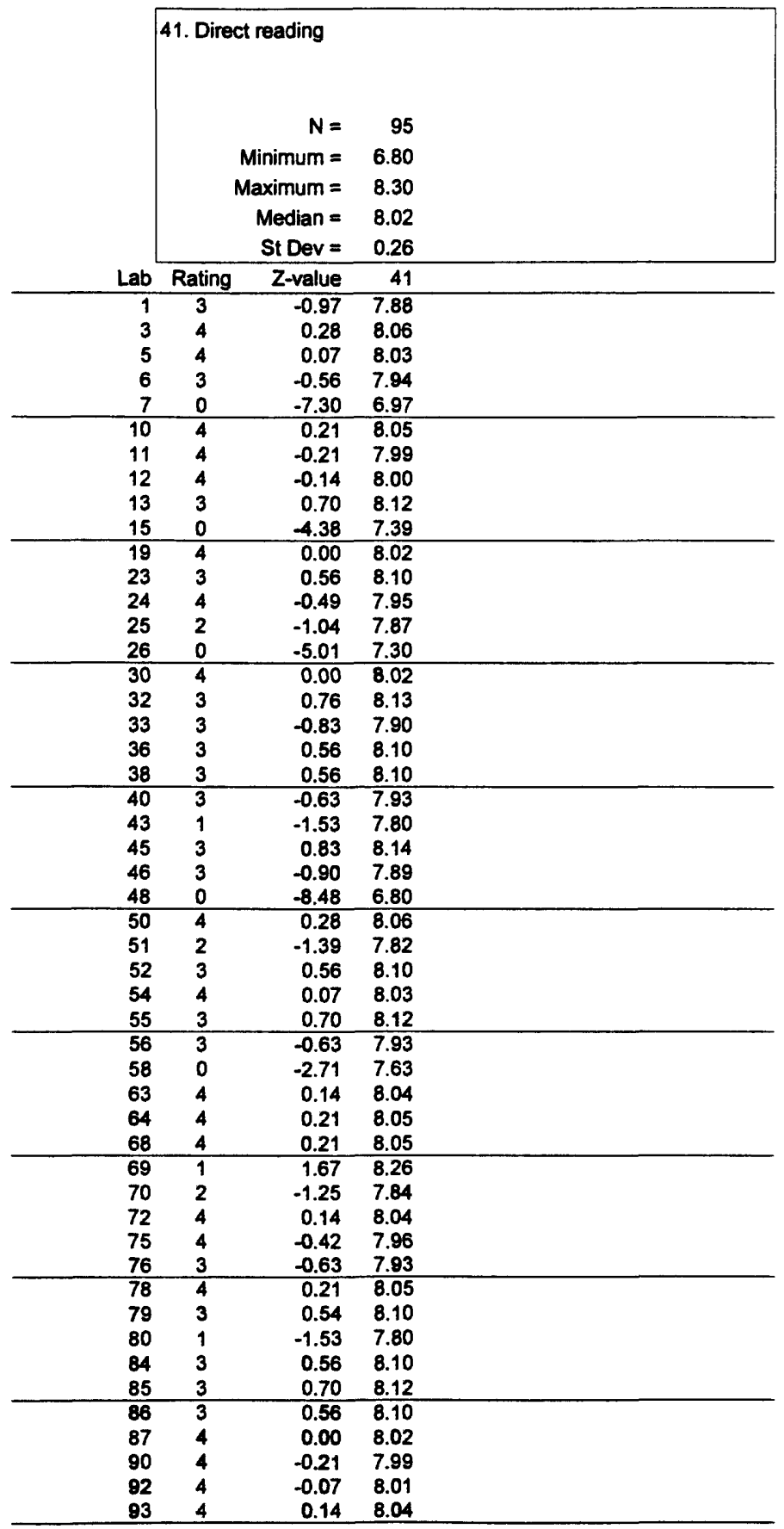

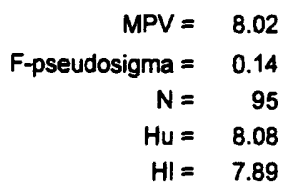

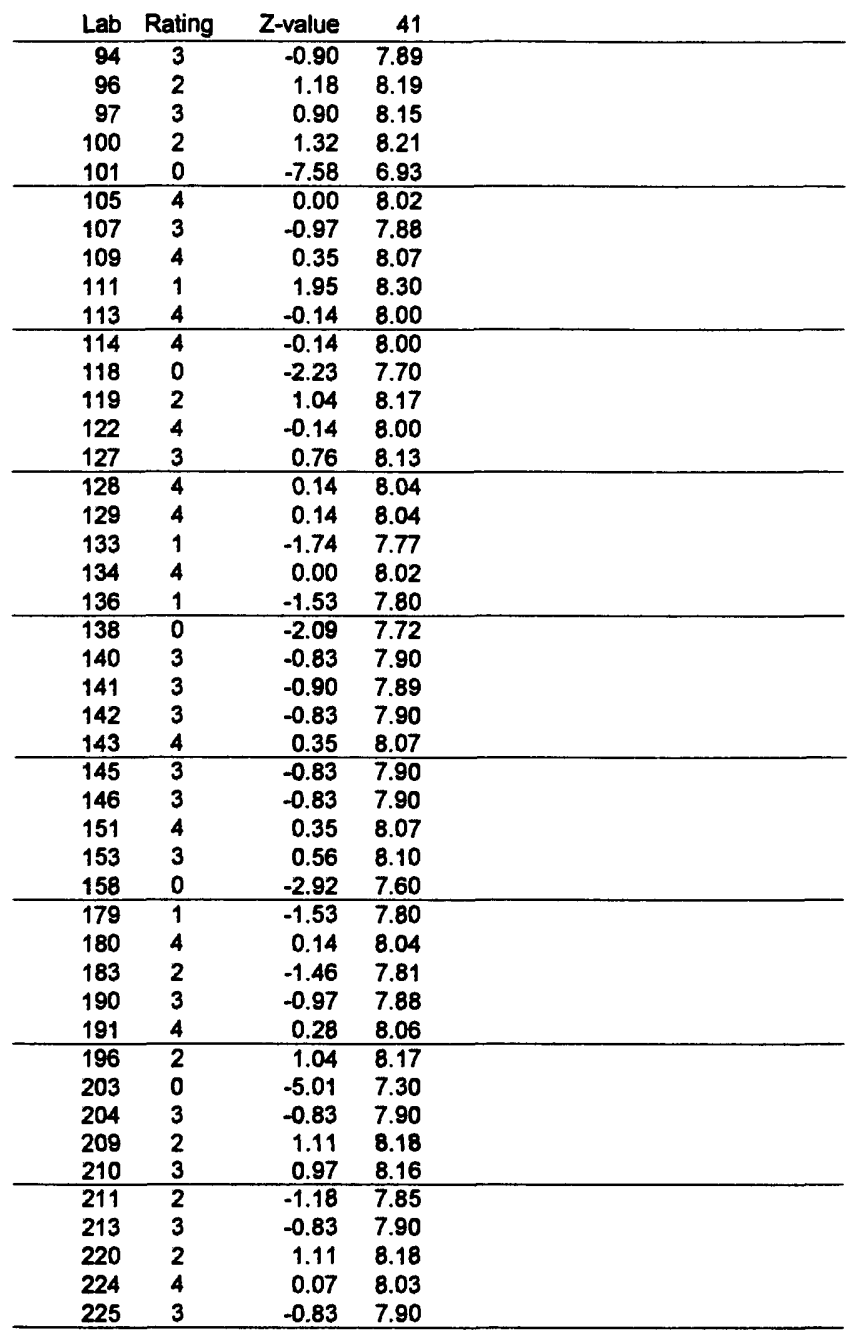


Table 11. -Statistical summary of reported data for standard reference water sample $M-130$ (major constituents)-Continued SiO2 (Silica) $\mathrm{m} \mathrm{g} / \mathrm{L}$

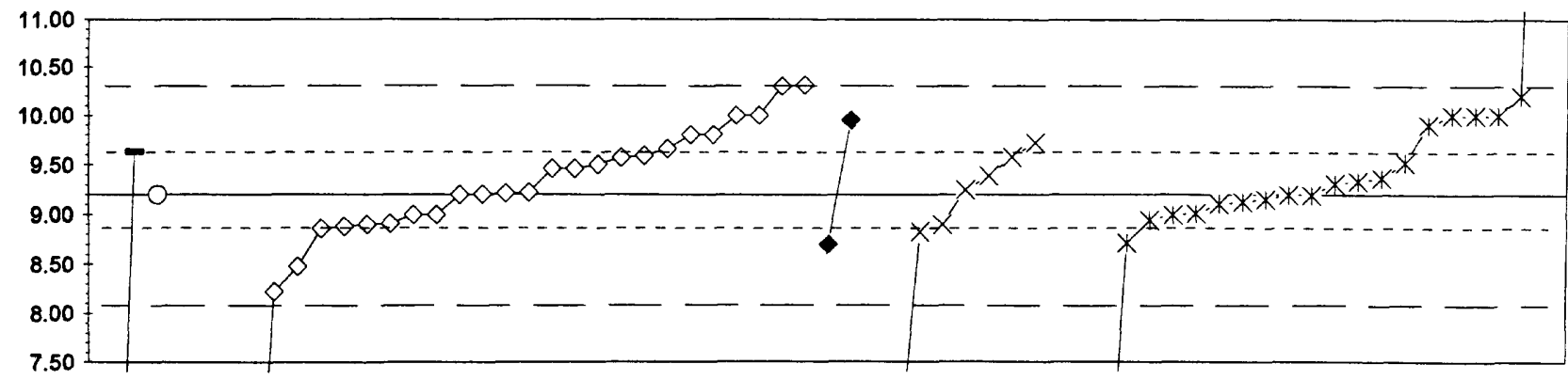

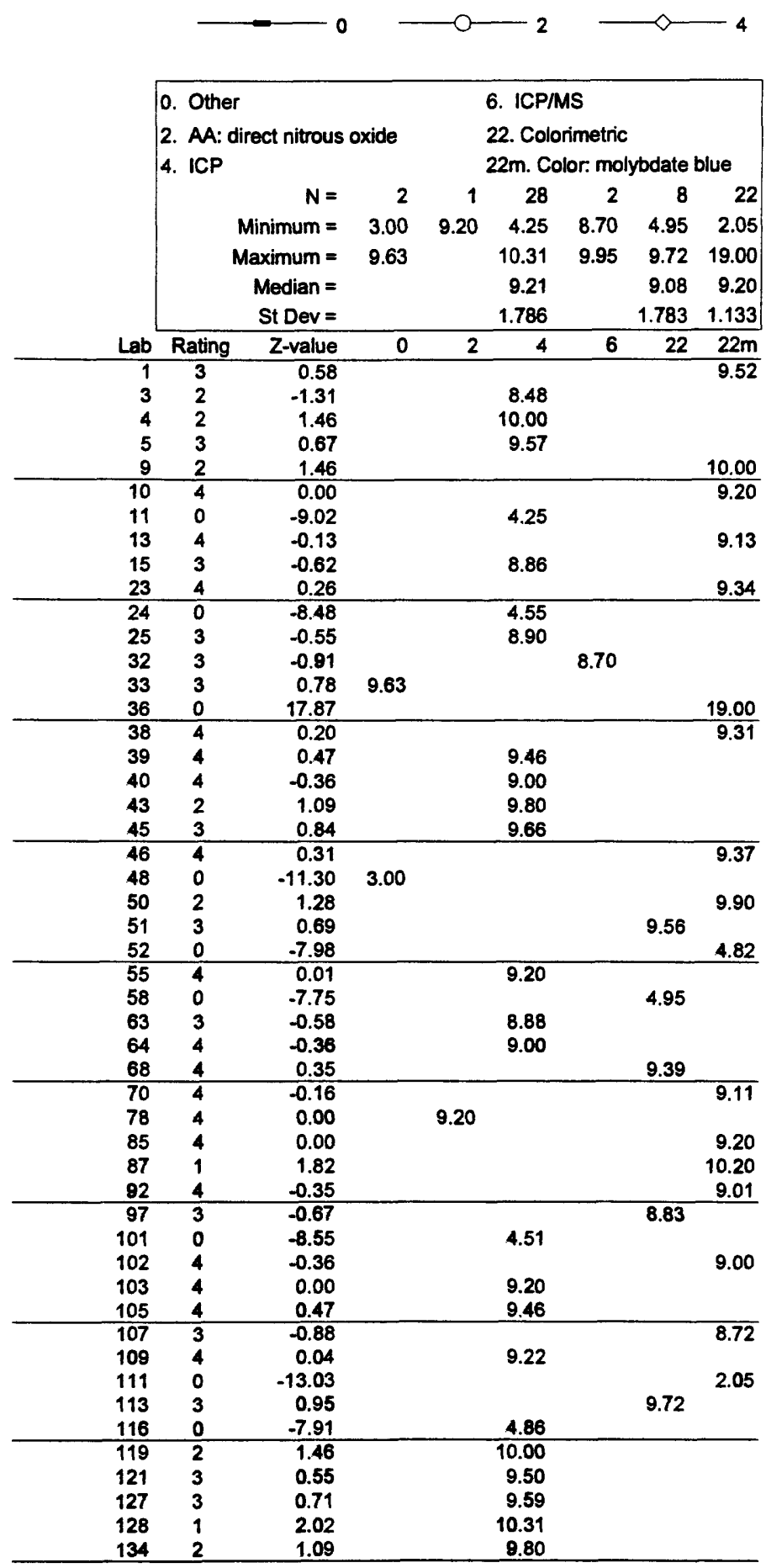

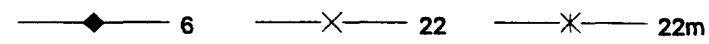

\begin{tabular}{|c|c|c|c|c|c|c|c|c|}
\hline Lab & Rating & Z-value & 0 & 2 & 4 & 6 & 22 & $22 \mathrm{~m}$ \\
\hline 138 & 2 & 1.46 & & & & & & 10.00 \\
\hline 140 & 4 & 0.09 & & & & & 9.25 & \\
\hline 141 & 4 & -0.47 & & & & & & 8.94 \\
\hline 142 & 4 & 0.03 & & & 9.22 & & & \\
\hline 145 & 3 & -0.53 & & & 8.91 & & & \\
\hline 146 & 1 & -1.79 & & & 8.22 & & & \\
\hline 191 & 2 & 1.37 & & & & 9.95 & & \\
\hline 203 & 4 & -0.07 & & & & & & 9.16 \\
\hline 204 & 2 & 1.46 & & & & & & 10.00 \\
\hline 209 & 3 & -0.55 & & & & & 8.90 & \\
\hline 210 & 1 & 2.01 & & & 10.30 & & & \\
\hline 211 & 0 & -5.58 & & & & & 6.14 & \\
\hline 224 & 0 & -9.72 & & & & & & 3.87 \\
\hline
\end{tabular}


Table 11. -Statistical summary of reported data for standard reference water sample M-130 (major constituents)-Continued SO4 (Sulfate) $\mathrm{m} \mathbf{g} / \mathbf{L}$

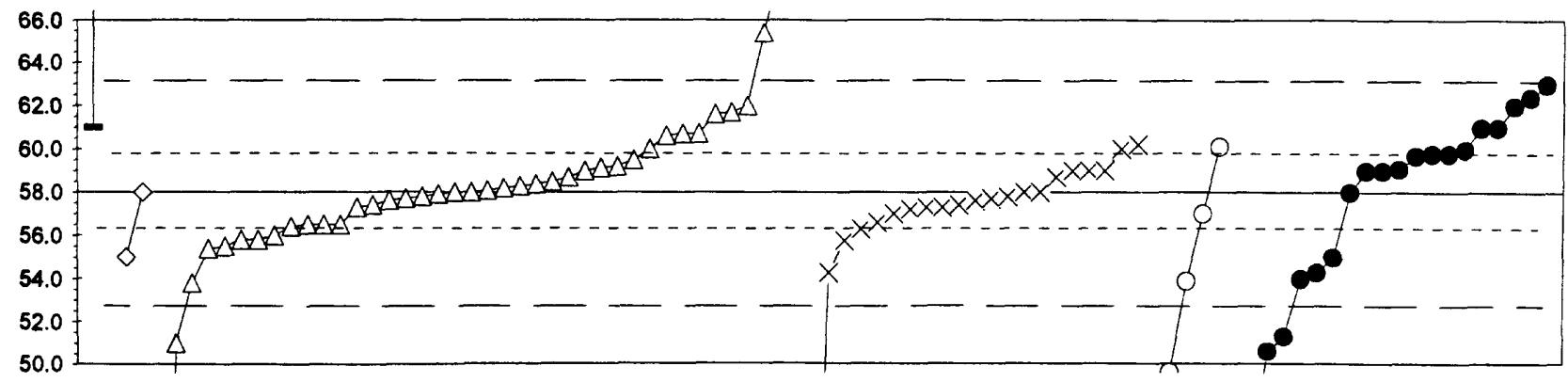

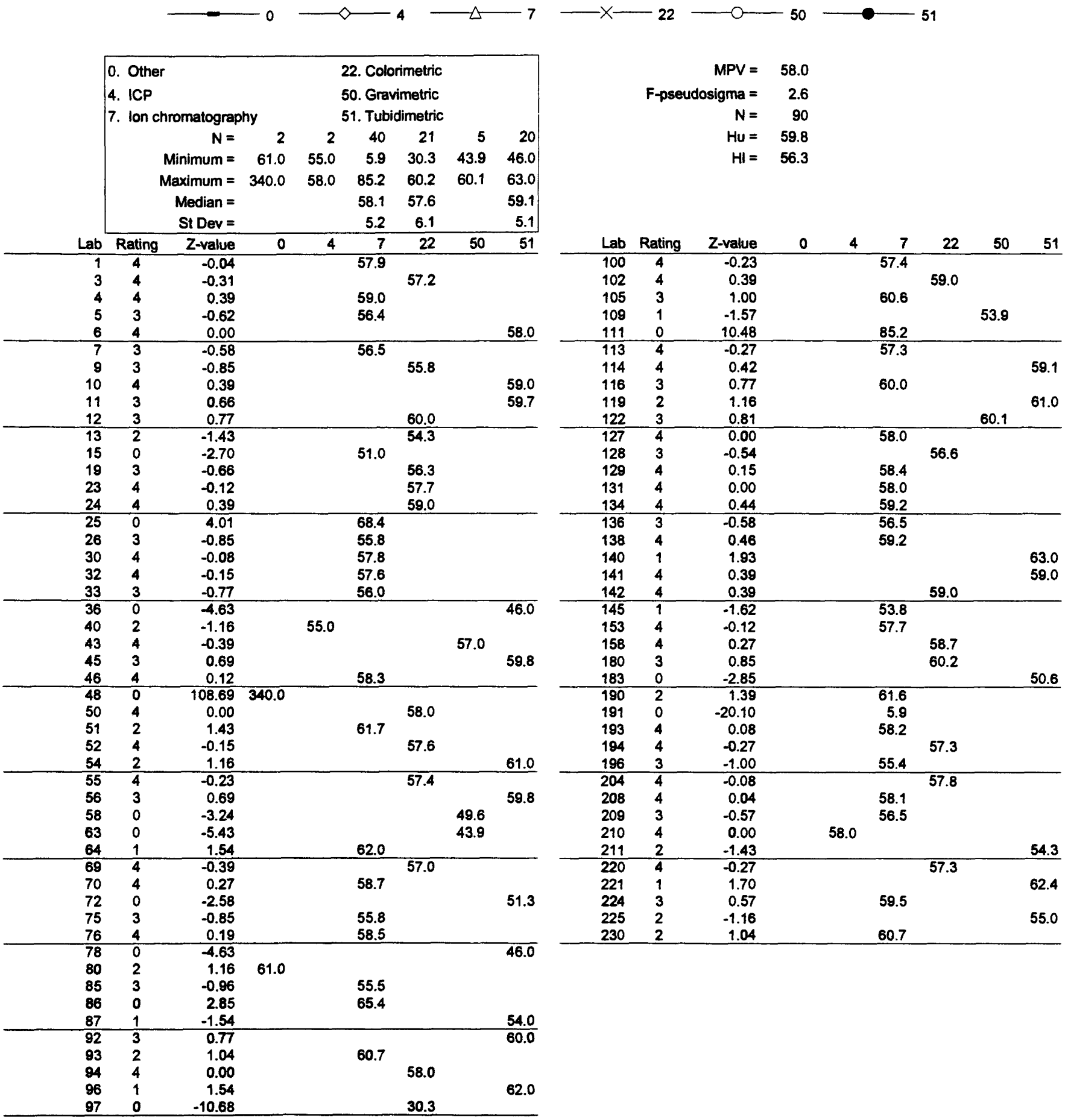


Table 11. -Statistical summary of reported data for standard reference water sample $M-130$ (major constituents)--Continued Sp Cond (Specific Conductance)

$\mu \mathrm{S} / \mathrm{cm}$
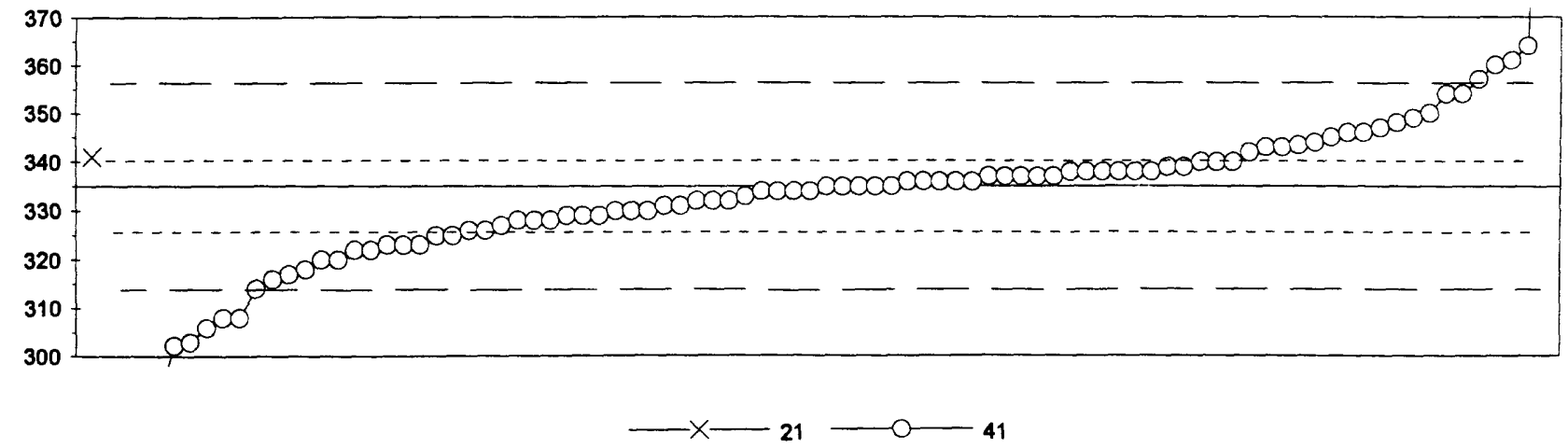

\begin{tabular}{|c|c|c|c|c|}
\hline \multirow[b]{3}{*}{ Lab } & \multicolumn{4}{|c|}{$\begin{array}{l}\text { 21. Titrate: electrometric } \\
\text { 41. Direct reading }\end{array}$} \\
\hline & \multicolumn{3}{|c|}{$\begin{array}{r}N= \\
\text { Minimum = } \\
\text { Maximum = } \\
\text { Median = } \\
\text { St Dev }=\end{array}$} & $\begin{array}{r}89 \\
37 \\
436 \\
335 \\
20\end{array}$ \\
\hline & Rating & Z-value & 21 & 41 \\
\hline $\begin{array}{l}1 \\
3 \\
5 \\
6 \\
7 \\
\end{array}$ & $\begin{array}{l}4 \\
4 \\
0 \\
2 \\
3\end{array}$ & $\begin{array}{r}0.10 \\
0.39 \\
-28.69 \\
1.45 \\
-0.58 \\
\end{array}$ & & $\begin{array}{r}336 \\
339 \\
37 \\
350 \\
329 \\
\end{array}$ \\
\hline 9 & 0 & -2.60 & & 308 \\
\hline 10 & 4 & 0.19 & & 337 \\
\hline 11 & 4 & -0.29 & & 332 \\
\hline 12 & 3 & 0.67 & & 342 \\
\hline 13 & 4 & 0.19 & & 337 \\
\hline 15 & 2 & 1.06 & & 346 \\
\hline 19 & 1 & -2.02 & & 314 \\
\hline 23 & 4 & 0.10 & & 336 \\
\hline 24 & 3 & -0.58 & & 329 \\
\hline 25 & 3 & 0.77 & & 343 \\
\hline 26 & 1 & 1.83 & & 354 \\
\hline 32 & 0 & -2.60 & & 308 \\
\hline 33 & 1 & -1.64 & & 318 \\
\hline 36 & 3 & 0.77 & & 343 \\
\hline 38 & 3 & 0.81 & & 343 \\
\hline 40 & 4 & 0.29 & & 338 \\
\hline 43 & 4 & 0.29 & & 338 \\
\hline 45 & 2 & 1.25 & & 348 \\
\hline 46 & 4 & -0.29 & & 332 \\
\hline 50 & 4 & 0.19 & & 337 \\
\hline 51 & 3 & $-0 . \overline{77}$ & & 327 \\
\hline 52 & 1 & -1.83 & & 316 \\
\hline 54 & 3 & -0.67 & & 328 \\
\hline 55 & 2 & 1.35 & & 349 \\
\hline 56 & 3 & -0.67 & & 328 \\
\hline 58 & 0 & -2.79 & & 306 \\
\hline 63 & 0 & -5.32 & & 280 \\
\hline 64 & 2 & 1.06 & & 346 \\
\hline 68 & 4 & 0.10 & & 336 \\
\hline 69 & 3 & 0.96 & & 345 \\
\hline 70 & 2 & -1.25 & & 322 \\
\hline 75 & 4 & 0.29 & & 338 \\
\hline 76 & 4 & -0.10 & & 334 \\
\hline 78 & 0 & -3.08 & & 303 \\
\hline 79 & 4 & -0.48 & & 330 \\
\hline 80 & 4 & -0.19 & & 333 \\
\hline 84 & 4 & 0.48 & & 340 \\
\hline 85 & 4 & -0.39 & & 331 \\
\hline 86 & 3 & -0.87 & & 326 \\
\hline 87 & 1 & -1.73 & & 317 \\
\hline 90 & 2 & -1.45 & & 320 \\
\hline 92 & 0 & 2.79 & & 364 \\
\hline 93 & 0 & -3.16 & & 302 \\
\hline 94 & 4 & 0.10 & & 336 \\
\hline 96 & 1 & 1.83 & & 354 \\
\hline
\end{tabular}

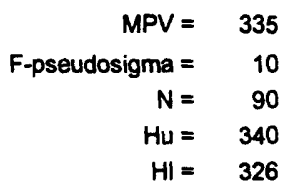

\begin{tabular}{|c|c|c|c|c|}
\hline Lab & Rating & Z-value & 21 & 41 \\
\hline 97 & 4 & 0.48 & & 340 \\
\hline 100 & 4 & -0.39 & & 331 \\
\hline 101 & 2 & -1.16 & & 323 \\
\hline 102 & 0 & 2.51 & & 361 \\
\hline 105 & 3 & 0.58 & 341 & \\
\hline 107 & 4 & 0.29 & & 338 \\
\hline 109 & 4 & 0.19 & & 337 \\
\hline 111 & 3 & -0.67 & & 328 \\
\hline 113 & 3 & 0.87 & & 344 \\
\hline 114 & 4 & 0.48 & & 340 \\
\hline 118 & 3 & -0.87 & & 326 \\
\hline 119 & 4 & -0.48 & & 330 \\
\hline 122 & 2 & -1.16 & & 323 \\
\hline 127 & 3 & -0.58 & & 329 \\
\hline 128 & 0 & 2.12 & & 357 \\
\hline 129 & 4 & 0.00 & & 335 \\
\hline 134 & 4 & 0.19 & & 337 \\
\hline 136 & 4 & 0.00 & & 335 \\
\hline 140 & 0 & -7.13 & & 261 \\
\hline 141 & 0 & 2.41 & & 360 \\
\hline 142 & 4 & -0.29 & & 332 \\
\hline 143 & 4 & 0.10 & & 336 \\
\hline 145 & 4 & -0.10 & & 334 \\
\hline 146 & 0 & -4.34 & & 290 \\
\hline 151 & 4 & 0.29 & & 338 \\
\hline 153 & 4 & 0.39 & & 339 \\
\hline 158 & 2 & 1.16 & & 347 \\
\hline 179 & 4 & 0.00 & & 335 \\
\hline 180 & 4 & -0.10 & & 334 \\
\hline 183 & 3 & -0.96 & & 325 \\
\hline 190 & 4 & 0.29 & & 336 \\
\hline 193 & 4 & 0.00 & & 335 \\
\hline 194 & 2 & -1.45 & & 320 \\
\hline 196 & 0 & 9.73 & & 436 \\
\hline 203 & 2 & -1.25 & & 322 \\
\hline 210 & 2 & -1.16 & & 323 \\
\hline 211 & 4 & 0.00 & & 335 \\
\hline 220 & 4 & -0.10 & & 334 \\
\hline 224 & 3 & -0.96 & & 325 \\
\hline 225 & 4 & -0.48 & & 330 \\
\hline
\end{tabular}


Table 11. -Statistical summary of reported data for standard reference water sample $M-130$ (major constituents)--Continued $\mathrm{Sr}$ (Strontium) $\mu \mathrm{g} / \mathrm{L}$

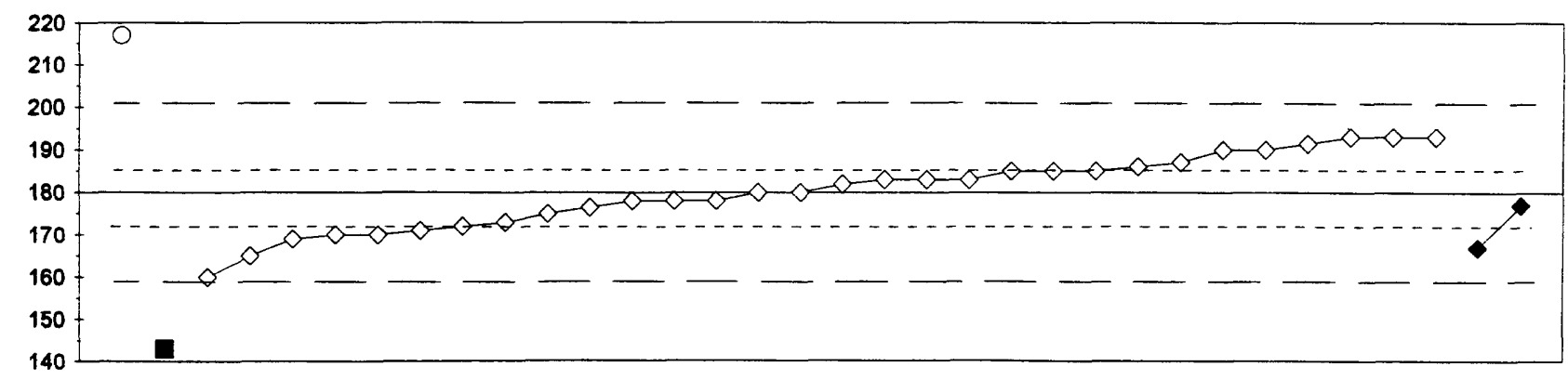

\begin{tabular}{|c|c|c|c|c|c|c|}
\hline \multirow[b]{2}{*}{ Lab } & $\begin{array}{l}\text { 2. AA: O } \\
\text { 3. AA: } \\
\text { 4. ICP }\end{array}$ & $\begin{array}{r}\text { lirect nitrous } \\
\text { graphite fum } \\
\qquad \begin{array}{r}N \\
\text { Minimum }\end{array}= \\
\text { Maximum }= \\
\text { Median }= \\
\text { StDev }=\end{array}$ & $\begin{array}{l}\text { xide } \\
\text { ce } \\
1 \\
217\end{array}$ & $\begin{array}{r}1 \\
143\end{array}$ & $\begin{array}{r}30 \\
160 \\
193 \\
181 \\
8.7\end{array}$ & $\begin{array}{r}2 \\
167 \\
177\end{array}$ \\
\hline & Rating & Z-value & 2 & 3 & 4 & 6 \\
\hline $\begin{array}{l}1 \\
3 \\
4 \\
5 \\
7\end{array}$ & $\begin{array}{l}4 \\
4 \\
2 \\
2 \\
3\end{array}$ & $\begin{array}{r}-0.34 \\
0.29 \\
1.25 \\
1.25 \\
0.58\end{array}$ & & & $\begin{array}{l}177 \\
183 \\
193 \\
193 \\
186\end{array}$ & \\
\hline $\begin{array}{l}11 \\
15 \\
24 \\
25 \\
32\end{array}$ & $\begin{array}{l}3 \\
3 \\
3 \\
4 \\
4\end{array}$ & $\begin{array}{l}0.96 \\
-0.87 \\
-0.67 \\
-0.48 \\
-0.29\end{array}$ & & & $\begin{array}{l}190 \\
171 \\
173 \\
175\end{array}$ & 177 \\
\hline $\begin{array}{l}39 \\
40 \\
46 \\
52 \\
55\end{array}$ & $\begin{array}{l}4 \\
3 \\
4 \\
1 \\
3\end{array}$ & $\begin{array}{r}0.48 \\
-0.96 \\
0.29 \\
-1.93 \\
-0.77\end{array}$ & & & $\begin{array}{l}185 \\
170 \\
183 \\
160 \\
172\end{array}$ & \\
\hline $\begin{array}{l}68 \\
70 \\
85 \\
94 \\
97\end{array}$ & $\begin{array}{l}3 \\
3 \\
4 \\
4 \\
0\end{array}$ & $\begin{array}{r}-0.96 \\
0.67 \\
0.00 \\
-0.19 \\
-3.57\end{array}$ & & 143 & $\begin{array}{l}170 \\
187 \\
180 \\
178\end{array}$ & \\
\hline $\begin{array}{l}102 \\
103 \\
105 \\
113 \\
116\end{array}$ & $\begin{array}{l}4 \\
4 \\
2 \\
0 \\
4\end{array}$ & $\begin{array}{r}0.19 \\
0.48 \\
1.25 \\
3.57 \\
-0.19\end{array}$ & 217 & & $\begin{array}{l}182 \\
185 \\
193 \\
\\
178\end{array}$ & \\
\hline $\begin{array}{l}121 \\
127 \\
134 \\
138 \\
142\end{array}$ & $\begin{array}{l}4 \\
4 \\
3 \\
4 \\
2\end{array}$ & $\begin{array}{l}-0.19 \\
0.29 \\
0.96 \\
0.48 \\
1.11\end{array}$ & & & $\begin{array}{l}178 \\
183 \\
190 \\
185 \\
192\end{array}$ & \\
\hline $\begin{array}{l}145 \\
146 \\
191 \\
194 \\
210\end{array}$ & \begin{tabular}{|c|}
2 \\
2 \\
2 \\
4 \\
NR
\end{tabular} & $\begin{array}{r}-1.06 \\
-1.45 \\
-1.25 \\
0.00\end{array}$ & & & $\begin{array}{r}169 \\
165 \\
\\
180 \\
<500 \\
\end{array}$ & 167 \\
\hline
\end{tabular}




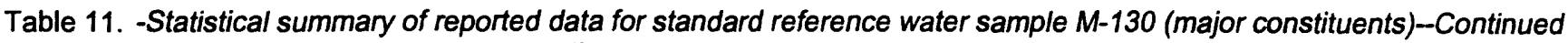
V (Vanadium) $\mu \mathrm{g} / \mathrm{L}$

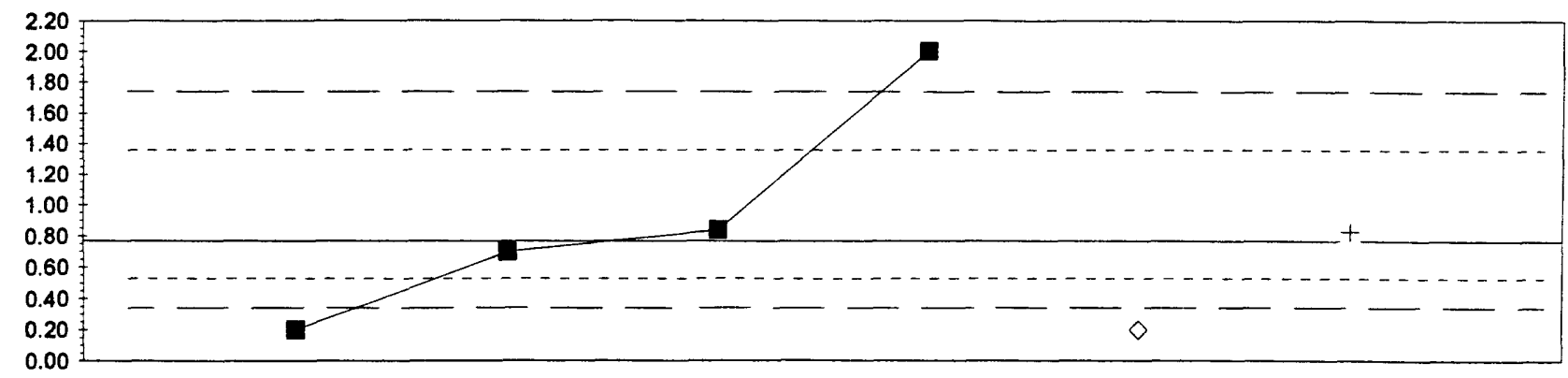

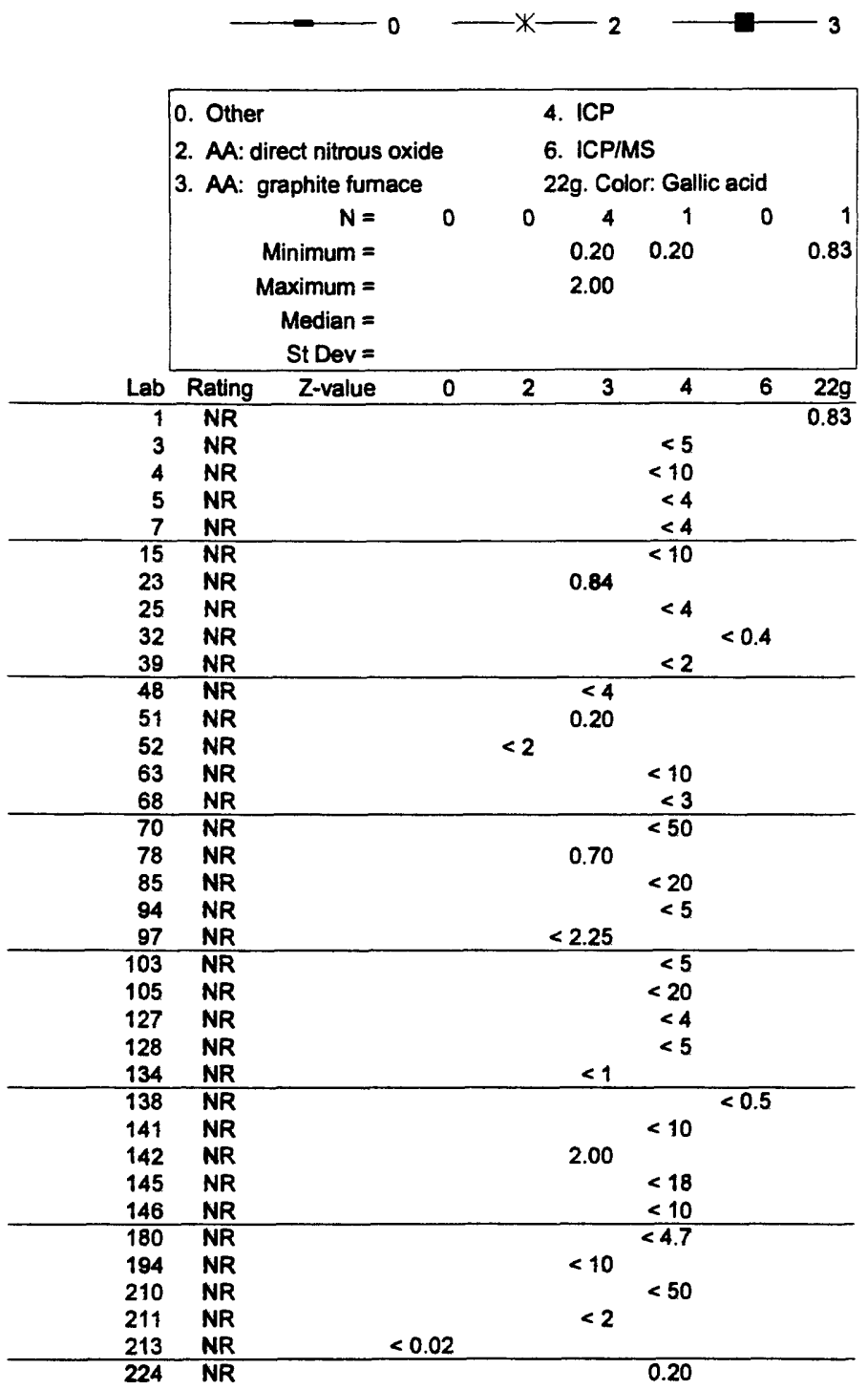


Table 12. -Statistical summary of reported data for standard reference water sample N-42 (nutrients)

Definition of analytical methods, abbreviations, and symbols.

Analytical methods

0. Other/Not reported

4. ICP

5. DCP

inductively coupled plasma

7. IC

direct current plasma

20. Titrate: color

ion chromatography

22: Color

titration: colorimetric [color reagent specified]

40. Ion selective electrode

colorimetric [color reagent specified]

\begin{tabular}{|c|c|c|}
\hline Abbreviations and & \multicolumn{2}{|c|}{$\begin{aligned} \mathrm{N} & =\text { number of samples } \\
\text { Stdev } & =\text { traditional standard deviation } \\
\mathrm{MPV} & =\text { most probable value } \\
\text { F-pseudosigma } & =\text { nonparametric statistic deviation } \\
H u & =\text { upper hinge value } \\
H I & =\text { lower hinge value } \\
\mathrm{mg} / \mathrm{L} & =\text { milligrams per liter } \\
\mathrm{Lab} & =\text { Laboratory code number } \\
\mathrm{NR} & =\text { not rated, less than value reported } \\
< & =\text { less than }\end{aligned}$} \\
\hline Constituent & & page \\
\hline $\mathrm{NH3}$ as $\mathrm{N}$ & Ammonia as nitrogen & 77 \\
\hline $\mathrm{NH} 3+\mathrm{Org} \mathrm{N}$ as $\mathrm{N}$ & Ammonia plus organic nitrogen as nitroge & 79 \\
\hline $\mathrm{NO} 3+\mathrm{NO} 2$ as $\mathrm{N}$ & Nitrate plus nitrite as nitrogen & 81 \\
\hline total $P$ as $P$ & total phosphorus as phosphorus & 83 \\
\hline $\mathrm{PO} 4$ as $\mathrm{P}$ & orthophosphate as phosphorus & 85 \\
\hline
\end{tabular}


Table 12. -Statistical summary of reported data for standard reference water sample N-42 (preserved nutrient)-Continued NH3 as N (Ammonia) m g/L

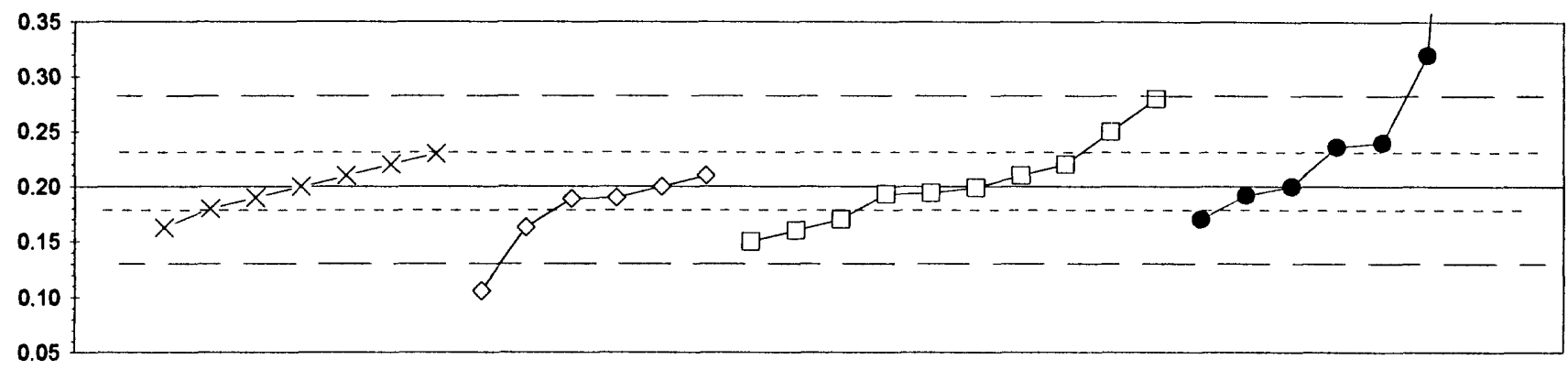

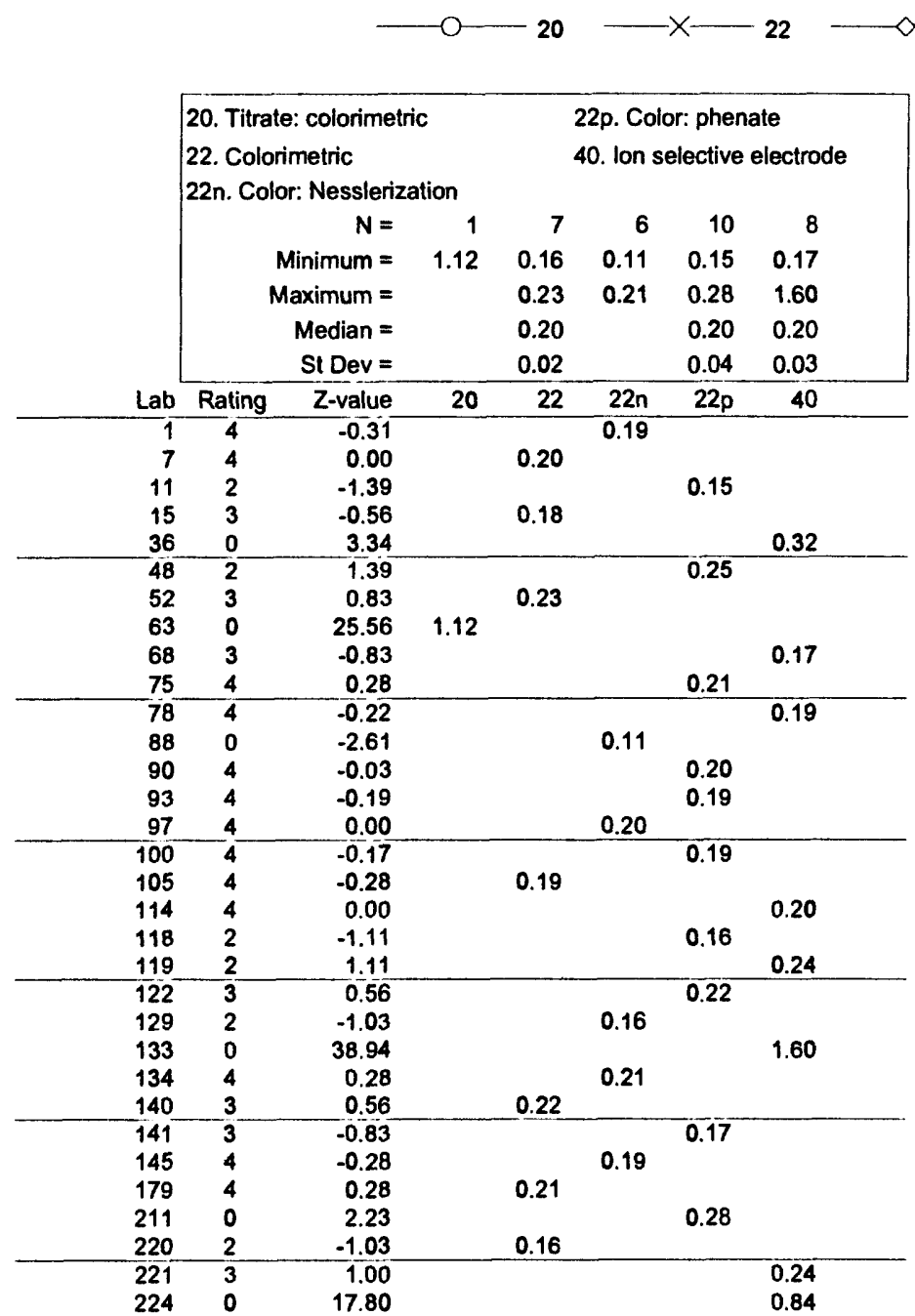


Table 12. -Statistical summary of reported data for standard reference water sample $\mathrm{N}-42$ (nonpreserved nutrient)--Continued NH3 as N (Ammonia)

$\mathrm{m} \mathrm{g/L}$

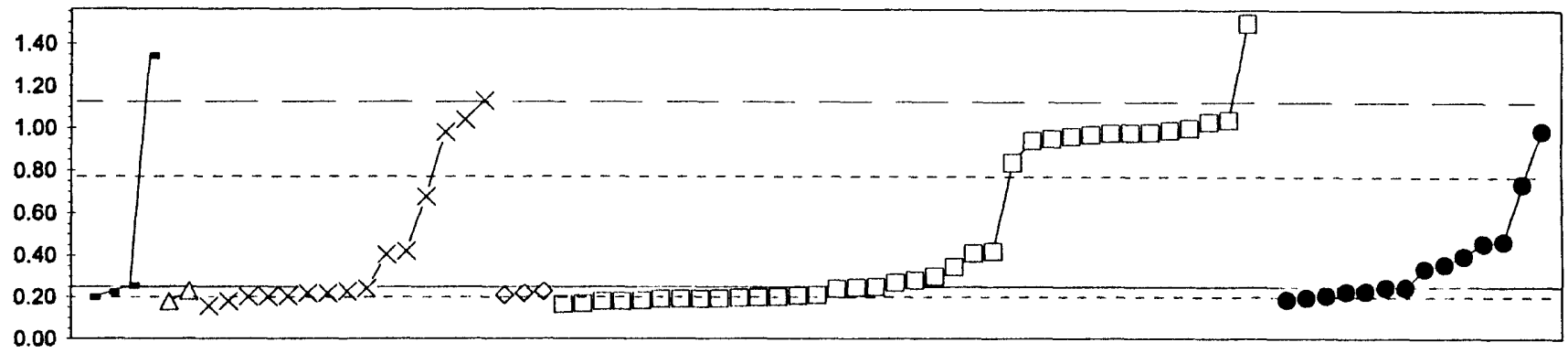

\begin{tabular}{|c|c|c|c|c|c|c|c|c|}
\hline & \multicolumn{3}{|c|}{$\begin{array}{l}\text { 0. Other } \\
\text { 7. Ion chromatography } \\
\text { 22. Colorimetric }\end{array}$} & \multicolumn{5}{|c|}{$\begin{array}{l}\text { 22n. Color: Nesslenization } \\
\text { 22p. Color: phenate } \\
\text { 40. Ion selective electrode }\end{array}$} \\
\hline \multirow[b]{6}{*}{ Lab } & & $\mathbf{N}=$ & 4 & 2 & 15 & 3 & 36 & 14 \\
\hline & & Minimum $=$ & 0.20 & 0.18 & 0.16 & 0.21 & 0.16 & 0.19 \\
\hline & & Maximum = & 1.34 & 0.23 & 1.13 & 0.23 & 1.50 & 0.99 \\
\hline & & Median $=$ & & & 0.23 & & 0.27 & 0.30 \\
\hline & & St Dev = & & & 0.35 & & 0.36 & 0.23 \\
\hline & Rating & Z-value & 0 & 7 & 22 & $22 n$ & $22 p$ & 40 \\
\hline 3 & 4 & 0.36 & & & 0.41 & & & \\
\hline 5 & 1 & 1.67 & & & & & 0.98 & \\
\hline 6 & 2 & 1.13 & & & & & & 0.74 \\
\hline 9 & 4 & 0.07 & & & & & 0.28 & \\
\hline 10 & 4 & -0.04 & & & & & & 0.23 \\
\hline 11 & 4 & -0.02 & & & & & 0.24 & \\
\hline 12 & NR & & & & & & $<0.2$ & \\
\hline 13 & 4 & 0.37 & & & & & 0.41 & \\
\hline 15 & 4 & -0.21 & & & 0.16 & & & \\
\hline 19 & 4 & 0.00 & & & & & 0.25 & \\
\hline 23 & 4 & -0.18 & & & & & 0.17 & \\
\hline 25 & 4 & 0.21 & & & & & & 0.34 \\
\hline 26 & 4 & -0.11 & & & 0.20 & & & \\
\hline 32 & 4 & -0.04 & 0.20 & & & & & \\
\hline 33 & 1 & 1.81 & & & 1.04 & & & \\
\hline 36 & 4 & 0.26 & & & & & & 0.36 \\
\hline 38 & 4 & -0.10 & & & & & 0.21 & \\
\hline 46 & 2 & 1.35 & & & & & 0.84 & \\
\hline 51 & 4 & -0.09 & & & & & & 0.21 \\
\hline 52 & 4 & 0.39 & & & 0.42 & & & \\
\hline 54 & 1 & 1.70 & & & & & & 0.99 \\
\hline 58 & 4 & 0.00 & & & & & & 0.25 \\
\hline 59 & 4 & -0.13 & & & & & 0.19 & \\
\hline 61 & 4 & -0.07 & & & 0.22 & & & \\
\hline 63 & 4 & -0.06 & & 0.18 & & & & \\
\hline 64 & 4 & 0.05 & & & & & 0.27 & \\
\hline 68 & 4 & 0.35 & & & & & & 0.40 \\
\hline 70 & 4 & -0.11 & & & 0.20 & & & \\
\hline 72 & 4 & -0.07 & & & 0.22 & & & \\
\hline 75 & 1 & 1.63 & & & & & 0.96 & \\
\hline 76 & 1 & 1.61 & & & & & 0.95 & \\
\hline 78 & 4 & -0.04 & & & & & & 0.23 \\
\hline 60 & 1 & 2.02 & & & 1.13 & & & \\
\hline 61 & 4 & -0.04 & & & 0.23 & & & \\
\hline 84 & 4 & 0.12 & & & & & 0.30 & \\
\hline 85 & 1 & 1.72 & & & & & 1.00 & \\
\hline 67 & 4 & 0.39 & & & & & 0.42 & \\
\hline 86 & 4 & -0.19 & & & & & 0.16 & \\
\hline 91 & 4 & -0.13 & & & & & 0.19 & \\
\hline 92 & 4 & -0.11 & & & & & & 0.20 \\
\hline 94 & 1 & 1.79 & & & & & 1.03 & \\
\hline 96 & 1 & 1.68 & & & 0.98 & & & \\
\hline 97 & 4 & -0.09 & & & & 0.21 & & \\
\hline 100 & 1 & 1.67 & & & & & 0.98 & \\
\hline 102 & 0 & 2.87 & & & & & 1.50 & \\
\hline 107 & 4 & -0.15 & & & & & 0.18 & \\
\hline 108 & 4 & 0.48 & & & & & & 0.46 \\
\hline 111 & 1 & 1.70 & & & & & 0.99 & \\
\hline 114 & 3 & 0.51 & & & & & & 0.47 \\
\hline 116 & 4 & -0.16 & & & & & 0.18 & \\
\hline
\end{tabular}

\begin{tabular}{|c|c|c|c|c|c|c|c|c|}
\hline$\checkmark$ & $-22 n$ & $-[-$ & $-22 p$ & 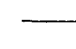 & 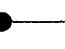 & 40 & & \\
\hline & & MPV $=$ & 0.25 & & & & & \\
\hline & F-pseu & osigma $=$ & 0.44 & & & & & \\
\hline & & $N=$ & 74 & & & & & \\
\hline & & $\mathrm{Hu}=$ & 0.79 & & & & & \\
\hline & & $H I=$ & 0.20 & & & & & \\
\hline Lab & Rating & Z-value & 0 & 7 & 22 & $22 n$ & $22 p$ & 40 \\
\hline 119 & 4 & 0.00 & & & & & & 0.25 \\
\hline 122 & 4 & -0.16 & & & & & 0.18 & \\
\hline 127 & 4 & 0.22 & & & & & 0.35 & \\
\hline 128 & 1 & 1.81 & & & & & 1.04 & \\
\hline 129 & 4 & -0.04 & & & & 0.23 & & \\
\hline 134 & 4 & -0.09 & & & & & 0.21 & \\
\hline 136 & 4 & -0.16 & 0.18 & & & & & \\
\hline 138 & 4 & -0.11 & & & & & 0.20 & \\
\hline 142 & 4 & -0.01 & & & 0.24 & & & \\
\hline 143 & 4 & -0.13 & & & & & 0.19 & \\
\hline 145 & 1 & 1.59 & & & & & 0.94 & \\
\hline 158 & 1 & 1.68 & & & & & 0.98 & \\
\hline 179 & 3 & 0.99 & & & 0.68 & & & \\
\hline 180 & 4 & -0.12 & & & & & 0.20 & \\
\hline 190 & 4 & 0.00 & & & & & 0.25 & \\
\hline 194 & 4 & -0.16 & & & 0.18 & & & \\
\hline 197 & 4 & -0.12 & & & & & 0.20 & \\
\hline 203 & 4 & -0.07 & & & & 0.22 & & \\
\hline 204 & 4 & -0.13 & & & & & 0.19 & \\
\hline 210 & 4 & -0.11 & 0.20 & & & & & \\
\hline 211 & 1 & 1.65 & & & & & 0.97 & \\
\hline 213 & 4 & 0.01 & 0.25 & & & & & \\
\hline 220 & 4 & -0.10 & & & 0.20 & & & \\
\hline 224 & 4 & -0.13 & & & & & & 0.19 \\
\hline 225 & 0 & 2.50 & & 1.34 & & & & \\
\hline
\end{tabular}


Table 12. -Statistical summary of reported data for standard reference water sample N-42 (preserved nutrient)-Continued $\mathrm{NH} 3+\operatorname{Org} \mathrm{N}$ as $\mathrm{N}$ (Ammonia + Organic N) $\mathrm{m} \mathrm{g} / \mathrm{L}$

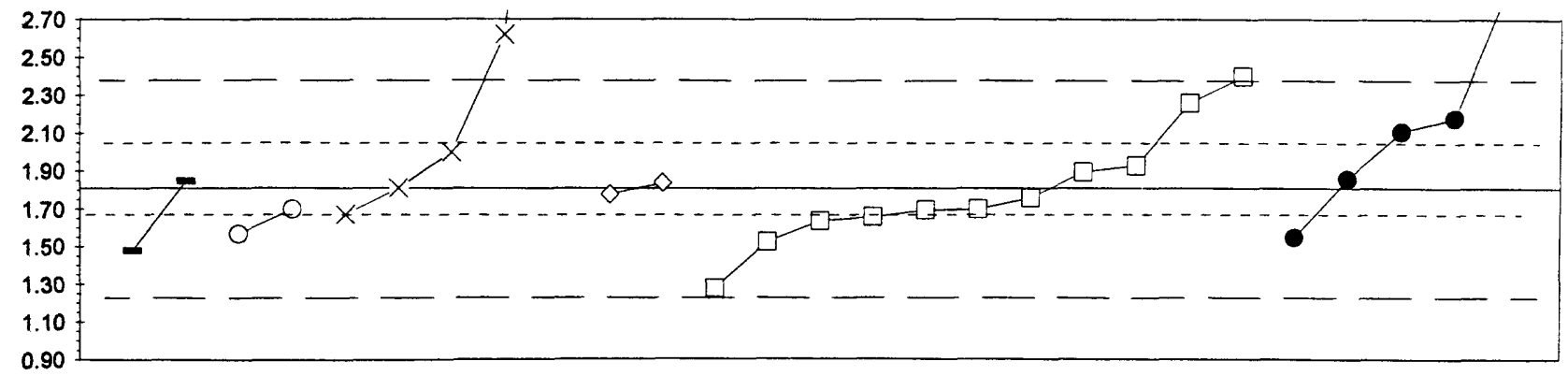

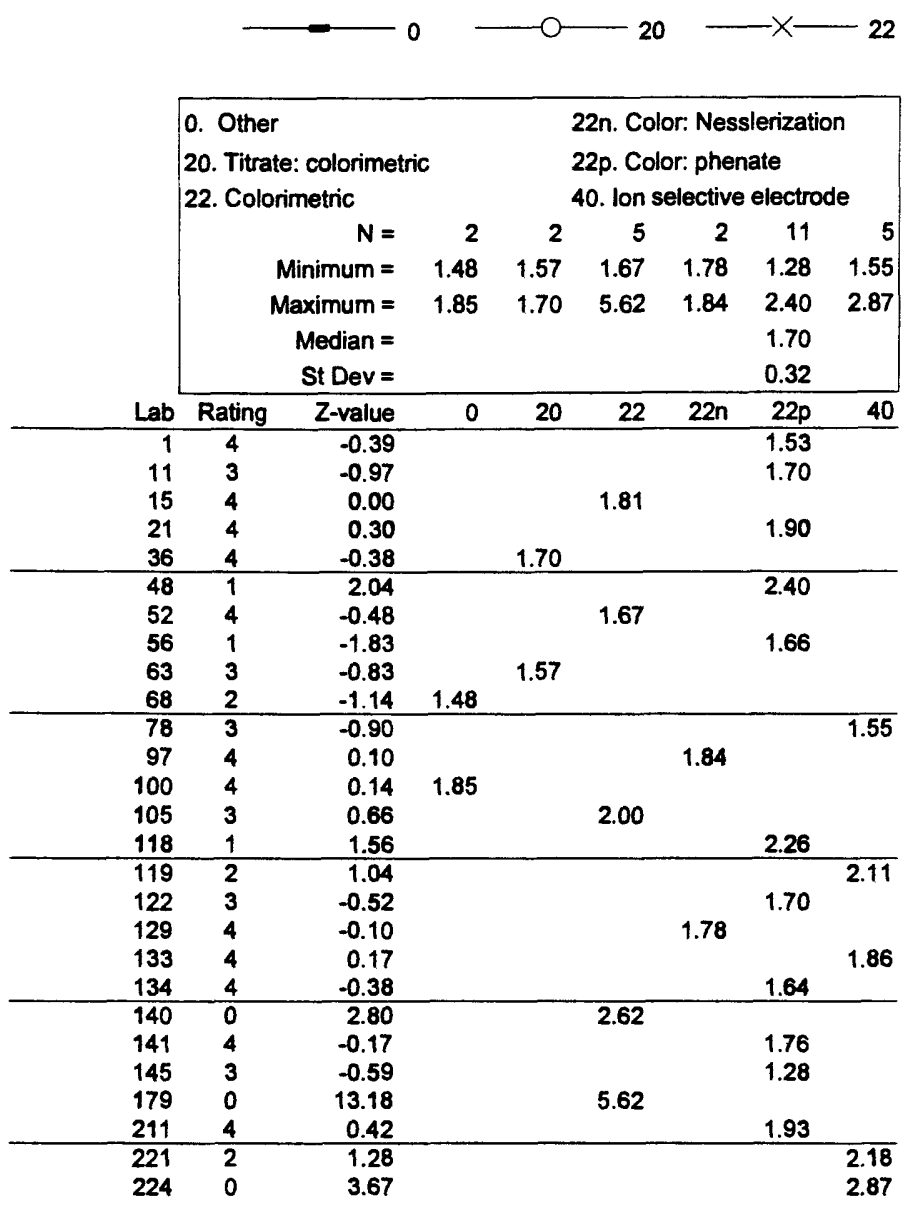


Table 12. -Statistical summary of reported data for standard reference water sample $\mathrm{N}-42$ (nonpreserved nutrient)--Continued NH3 + Org N as N (Ammonia + Organic N) $\mathrm{m} \mathbf{g} / \mathrm{L}$

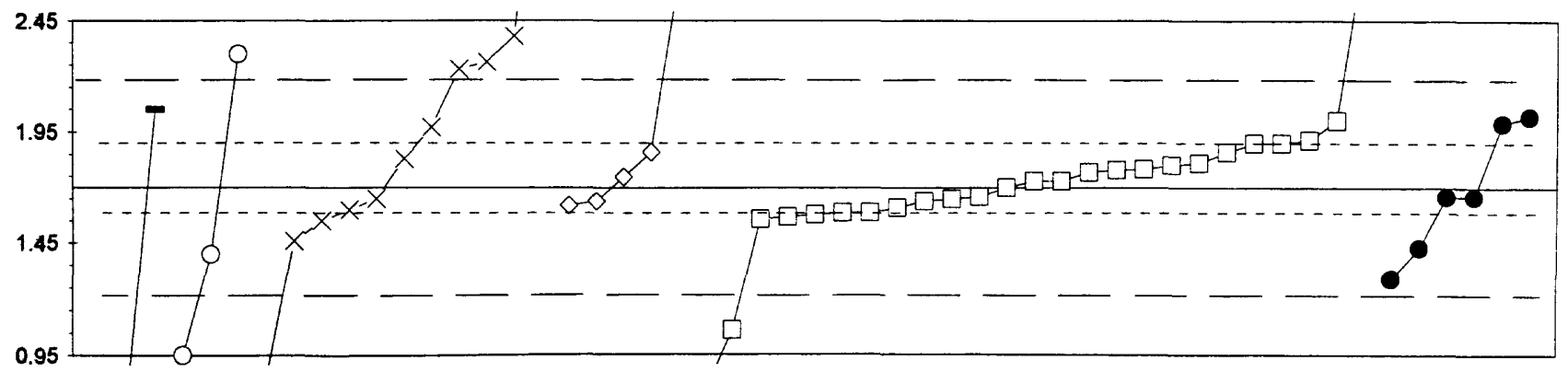

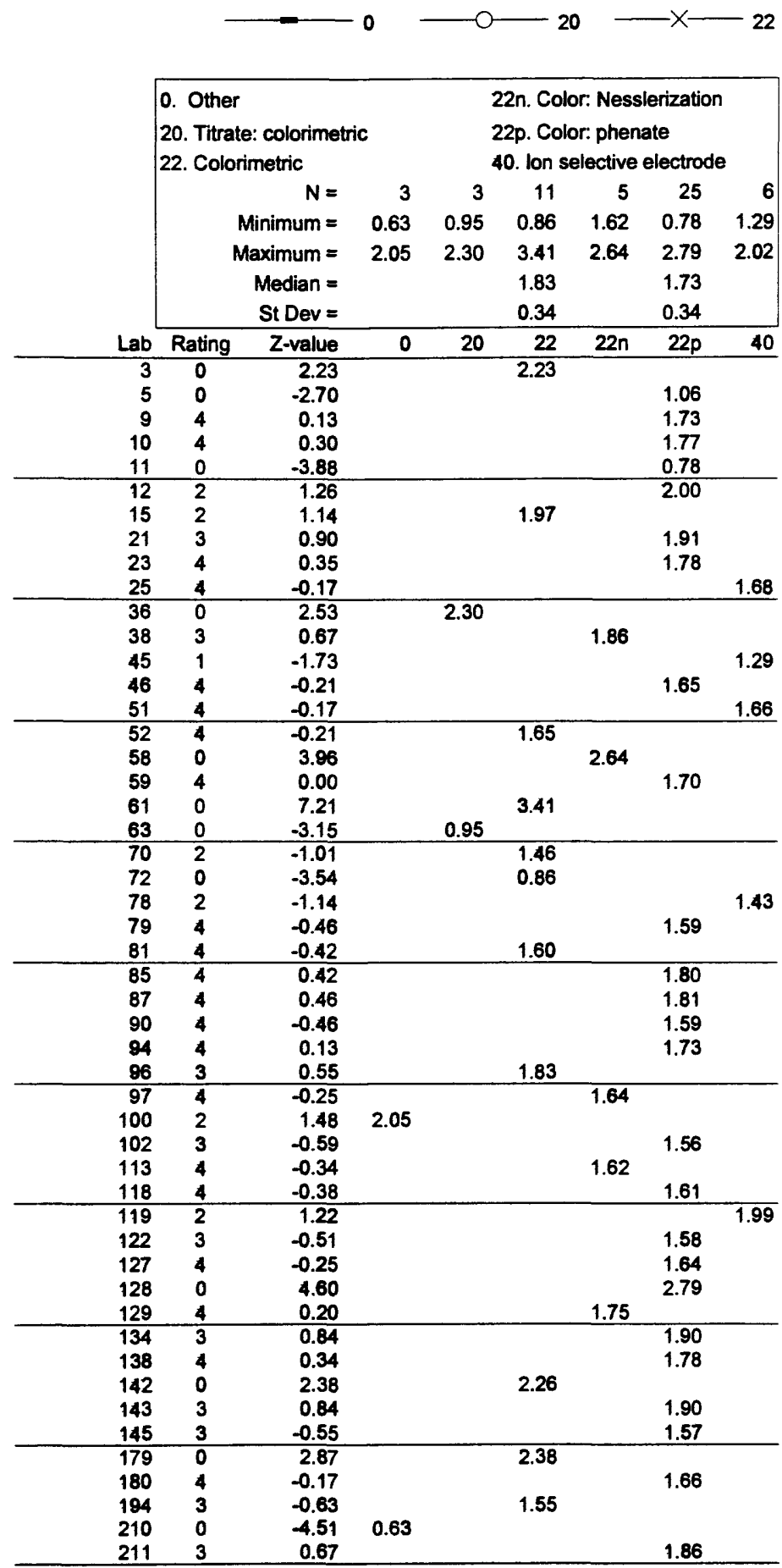

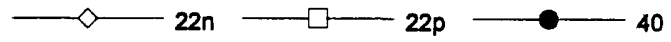

\begin{tabular}{rrrrrrrrr} 
Lab & Rating & Z-value & 0 & 20 & 22 & $22 n$ & $22 p$ & 40 \\
\hline 213 & 0 & -3.78 & 0.80 & & & & & \\
224 & 2 & 1.35 & & & & & & 2.02 \\
225 & 2 & -1.26 & & 1.40 & & & &
\end{tabular}


Table 12. -Statistical summary of reported data for standard reference water sample N-42 (preserved nutrient)-Continued $\mathrm{NO} 3+\mathrm{NO} 2$ as N (Nitrate + Nitrite)

$\mathrm{m}$ g/L

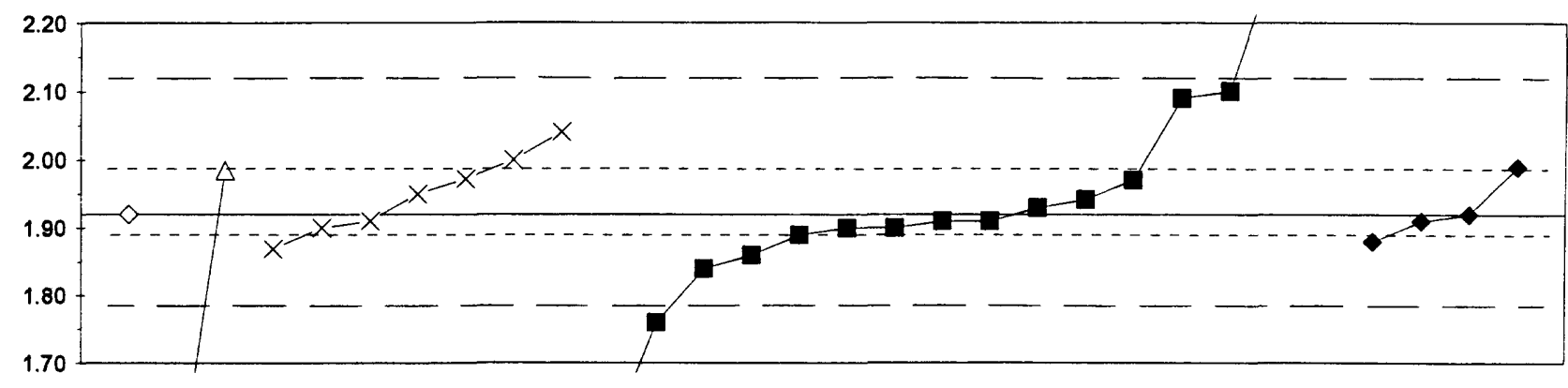

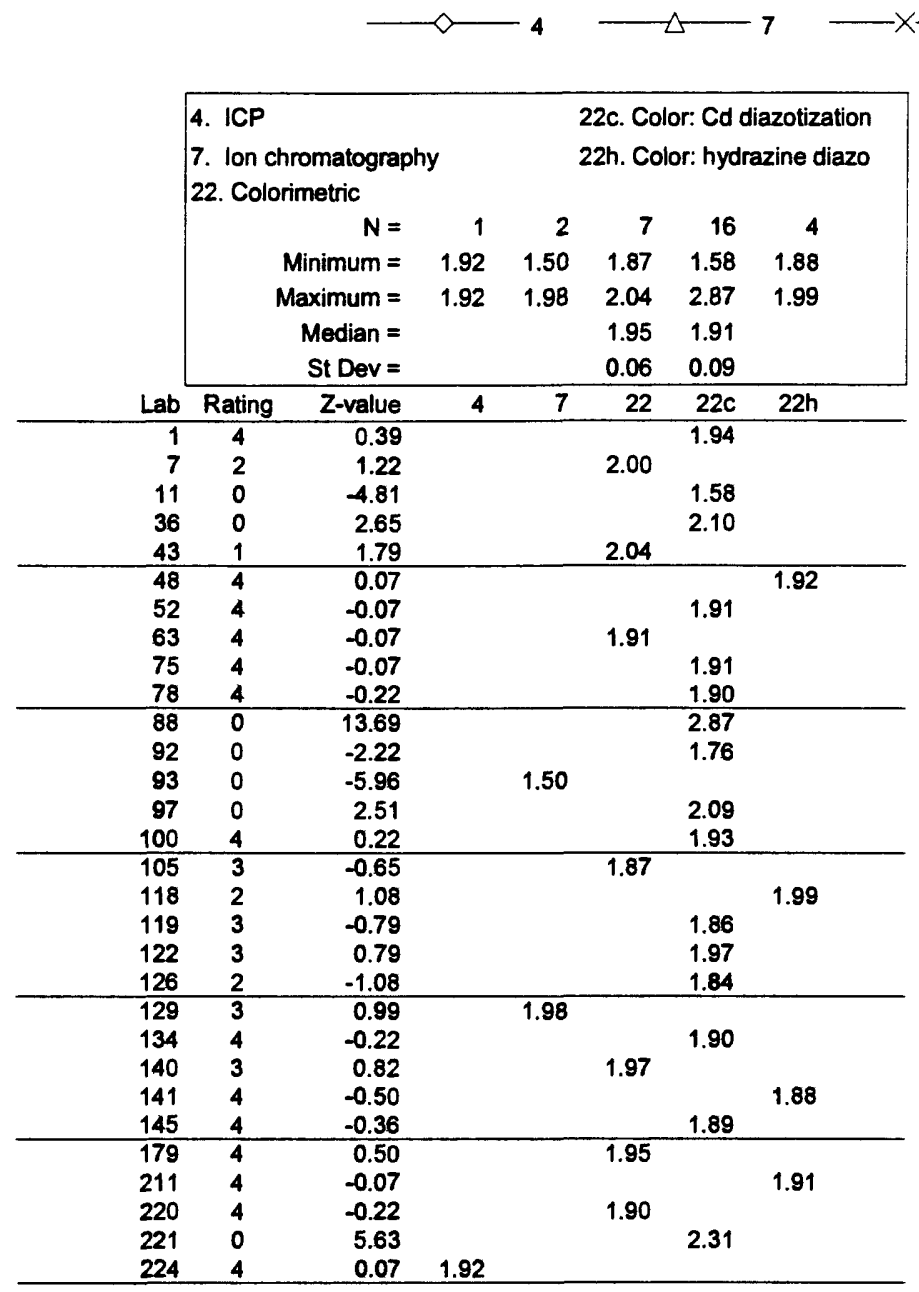


Table 12. -Statistical summary of reported data for standard reference water sample $\mathrm{N}-42$ (nonpreserved nutrient)-Continued $\mathrm{NO} 3$ + NO2 as N (Nitrate + Nitrite) $\mathrm{m}$ g/L

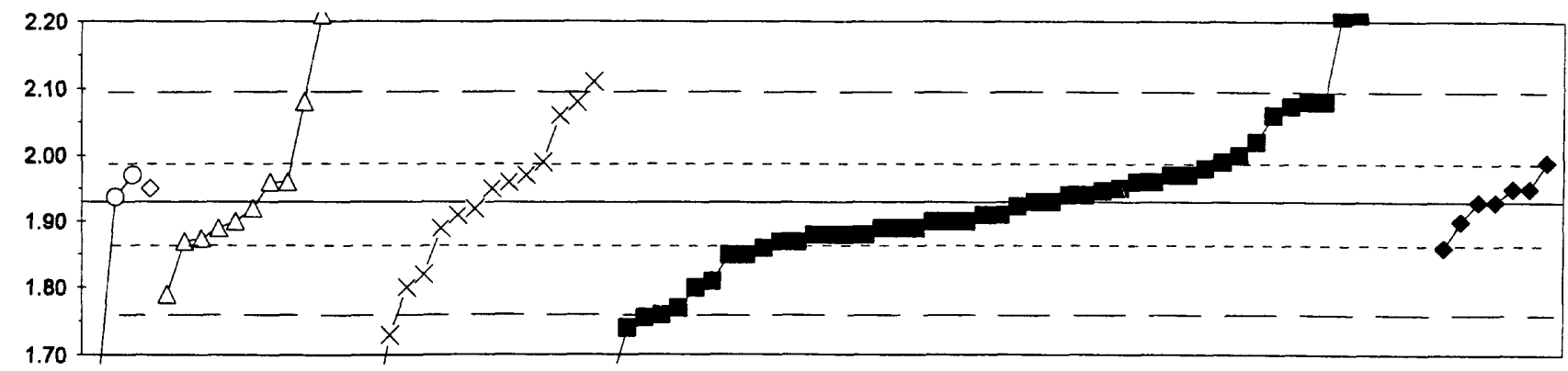

\begin{tabular}{|c|c|c|c|c|c|c|c|c|c|c|c|c|c|c|c|c|c|}
\hline \multirow[b]{9}{*}{ Lab } & \multicolumn{3}{|l|}{ o. Other } & \multicolumn{5}{|c|}{ 22. Colorimetric } & & & MPV $=$ & 1.93 & & & & & \\
\hline & \multicolumn{3}{|l|}{ 4. ICP } & \multicolumn{5}{|c|}{ 22c. Color: Cd diazotization } & \multicolumn{3}{|c|}{ F-pseudosigma = } & 0.08 & & & & & \\
\hline & \multicolumn{3}{|c|}{ 7. Ion chromatography } & \multicolumn{5}{|c|}{ 22h. Color: hydrazine diazo } & \multirow[b]{7}{*}{ Lab } & \multirow[b]{7}{*}{ Rating } & $N=$ & 86 & & & & & \\
\hline & & $N=$ & 3 & 1 & 11 & 15 & 49 & 7 & & & $\mathrm{Hu}=$ & 1.98 & & & & & \\
\hline & & Minimum = & 1.65 & 1.95 & 1.79 & 1.40 & 1.65 & 1.86 & & & $\mathrm{HI}=$ & 1.87 & & & & & \\
\hline & & Maximum = & 1.97 & 1.95 & 2.68 & 2.11 & 3.16 & 1.99 & & & & & & & & & \\
\hline & & Median = & & & 1.90 & 1.95 & 1.91 & 1.93 & & & & & & & & & \\
\hline & & St Dev = & & & 0.08 & 0.10 & 0.08 & 0.04 & & & & & & & & & \\
\hline & Rating & Z-value & 0 & 4 & 7 & 22 & $22 c$ & $22 \mathrm{~h}$ & & & Z-value & 0 & 4 & 7 & 22 & $22 c$ & $22 h$ \\
\hline 3 & 3 & 0.76 & & & & 1.99 & & & 108 & 0 & -2.55 & & & & 1.73 & & \\
\hline 5 & 4 & 0.00 & & & & & & 1.93 & 113 & 1 & -1.53 & & & & & 1.81 & \\
\hline 6 & 0 & 15.65 & & & & & 3.16 & & 114 & 2 & 1.15 & & & & & 2.02 & \\
\hline 9 & 4 & 0.25 & & & & & 1.95 & & 118 & 4 & 0.25 & & & & & & 1.95 \\
\hline 10 & 4 & 0.13 & & & & & 1.94 & & 119 & 3 & -0.89 & & & & & 1.86 & \\
\hline 11 & 3 & -0.51 & & & & & 1.89 & & 122 & 3 & 0.89 & & & & & 2.00 & \\
\hline 12 & 3 & 0.64 & & & & & 1.98 & & 127 & 4 & -0.25 & & & & & 1.91 & \\
\hline 13 & 4 & 0.00 & & & & & 1.93 & & 128 & 4 & 0.00 & & & & & & 1.93 \\
\hline 15 & 4 & 0.38 & & & & 1.96 & & & 129 & 3 & -0.71 & & & 1.87 & & & \\
\hline 19 & 1 & 1.65 & & & & & 2.06 & & 133 & 1 & 1.91 & & & & & 2.08 & \\
\hline 23 & 4 & -0.25 & & & & & 1.91 & & 134 & 4 & -0.38 & & & & & 1.90 & \\
\hline 25 & 0 & 9.54 & & & 2.68 & & & & 136 & 1 & 1.91 & & & 2.08 & & & \\
\hline 26 & 2 & -1.40 & & & & 1.82 & & & 138 & 3 & -0.76 & & & & & 1.87 & \\
\hline 32 & 4 & 0.38 & & & 1.96 & & & & 142 & 0 & -6.75 & & & & 1.40 & & \\
\hline 36 & 4 & -0.38 & & & & & 1.90 & & 143 & 3 & 0.51 & & & & & 1.97 & \\
\hline 38 & 2 & -1.02 & & & & & 1.85 & & 145 & 3 & -0.51 & & & & & 1.89 & \\
\hline 45 & 3 & 0.76 & & & & & 1.99 & & 146 & 0 & -2.16 & & & & & 1.76 & \\
\hline 46 & 4 & -0.09 & & & & & 1.92 & & 158 & 4 & 0.25 & & & & & & 1.95 \\
\hline 51 & 0 & 3.56 & & & 2.21 & & & & 179 & 3 & 0.51 & & & & 1.97 & & \\
\hline 52 & 3 & -0.64 & & & & & 1.88 & & 180 & 4 & 0.00 & & & & & 1.93 & \\
\hline 53 & 0 & 3.53 & & & & & 2.21 & & 183 & 0 & -3.56 & 1.65 & & & & & \\
\hline 55 & 4 & 0.38 & & & & & 1.96 & & 190 & 0 & 3.92 & & & & & 2.24 & \\
\hline 56 & 0 & -2.42 & & & & & 1.74 & & 191 & 3 & -0.51 & & & 1.89 & & & \\
\hline 58 & 1 & -1.78 & & & 1.79 & & & & 193 & 4 & 0.38 & & & 1.96 & & & \\
\hline 59 & 3 & -0.64 & & & & & 1.88 & & 194 & 0 & 2.29 & & & & 2.11 & & \\
\hline 61 & 4 & 0.25 & & & & 1.95 & & & 196 & 3 & -0.76 & & & 1.87 & & & \\
\hline 63 & 1 & -1.65 & & & & 1.80 & & & 197 & 4 & 0.20 & & & & & 1.95 & \\
\hline 64 & 3 & 0.51 & & & & & 1.97 & & 203 & 0 & -3.56 & & & & & 1.65 & \\
\hline 68 & 3 & 0.51 & 1.97 & & & & & & 204 & 4 & 0.38 & & & & & 1.96 & \\
\hline 69 & 3 & -0.64 & & & & & 1.88 & & 205 & 0 & 8.78 & & & & & 2.62 & \\
\hline 70 & 3 & -0.51 & & & & 1.89 & & & 208 & 4 & -0.13 & & & 1.92 & & & \\
\hline 72 & 1 & 1.91 & & & & 2.08 & & & 210 & 4 & 0.09 & 1.94 & & & & & \\
\hline 75 & 3 & -0.51 & & & & & 1.89 & & 211 & 4 & -0.38 & & & & & & 1.90 \\
\hline 76 & 4 & -0.38 & & & 1.90 & & & & 220 & 1 & 1.65 & & & & 2.06 & & \\
\hline 78 & 1 & -1.65 & & & & & 1.80 & & 224 & 4 & 0.25 & & 1.95 & & & & \\
\hline 80 & 0 & -4.45 & & & & 1.58 & & & 225 & 0 & 3.56 & & & & & 2.21 & \\
\hline 81 & 4 & -0.25 & & & & 1.91 & & & & & & & & & & & \\
\hline 84 & $i$ & -2.04 & & & & & 1.77 & & & & & & & & & & \\
\hline 85 & 4 & -0.38 & & & & & 1.90 & & & & & & & & & & \\
\hline 87 & 3 & -0.76 & & & & & 1.87 & & & & & & & & & & \\
\hline 88 & 0 & 11.21 & & & & & 2.81 & & & & & & & & & & \\
\hline 90 & 3 & 0.76 & & & & & & 1.99 & & & & & & & & & \\
\hline 91 & 3 & -0.89 & & & & & & 1.86 & & & & & & & & & \\
\hline 92 & 0 & -2.23 & & & & & 1.76 & & & & & & & & & & \\
\hline 94 & 2 & -1.02 & & & & & 1.85 & & & & & & & & & & \\
\hline 96 & 4 & -0.13 & & & & 1.92 & & & & & & & & & & & \\
\hline 97 & 1 & 1.91 & & & & & 2.08 & & & & & & & & & & \\
\hline 100 & 4 & 0.13 & & & & & 1.94 & & & & & & & & & & \\
\hline 102 & 3 & -0.64 & & & & & 1.88 & & & & & & & & & & \\
\hline 107 & 1 & 1.82 & & & & & 2.07 & & & & & & & & & & \\
\hline
\end{tabular}


Table 12. -Statistical summary of reported data for standard reference water sample N-42 (preserved nutrient)-Continued total P as P (total Phosphorus)

m g/L
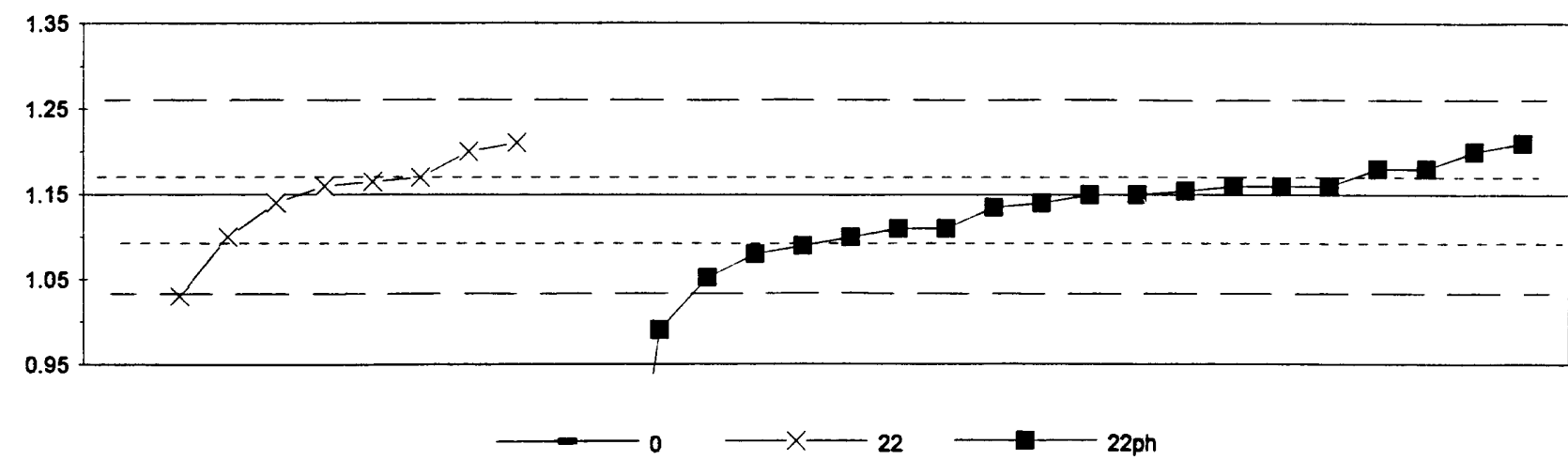

\begin{tabular}{|c|c|c|c|c|c|}
\hline \multirow[b]{7}{*}{ Lab } & \multicolumn{5}{|c|}{$\begin{array}{l}\text { 0. Other } \\
\text { 22. Colorimetric } \\
\text { 22ph. Color: phosphomolybdate }\end{array}$} \\
\hline & & $\mathbf{N}=$ & 1 & 8 & 21 \\
\hline & & Minimum = & 0.05 & 1.03 & 0.59 \\
\hline & \multicolumn{3}{|c|}{ Maximum = } & 1.21 & 1.21 \\
\hline & \multicolumn{3}{|c|}{ Median = } & 1.16 & 1.15 \\
\hline & \multicolumn{3}{|c|}{ St Dev = } & 0.06 & 0.05 \\
\hline & Rating & Z-value & 0 & 22 & 22ph \\
\hline 1 & 4 & 0.16 & & & 1.15 \\
\hline 7 & 4 & 0.45 & & 1.17 & \\
\hline 11 & 0 & -9.80 & & & 0.60 \\
\hline 15 & 3 & -0.81 & & 1.10 & \\
\hline 36 & 0 & -2.79 & & & 0.99 \\
\hline 48 & 3 & -0.81 & & & 1.10 \\
\hline 52 & 4 & 0.09 & & & 1.15 \\
\hline 56 & 1 & -1.67 & & & 1.05 \\
\hline 63 & 3 & 0.99 & & 1.20 & \\
\hline 68 & 0 & -19.73 & 0.05 & & \\
\hline 75 & 4 & -0.09 & & & 1.14 \\
\hline 78 & 2 & 1.17 & & & 1.21 \\
\hline 92 & 0 & -9.96 & & & 0.59 \\
\hline 97 & 3 & -0.63 & & & 1.11 \\
\hline 100 & 3 & -0.63 & & & 1.11 \\
\hline 105 & 4 & 0.27 & & 1.16 & \\
\hline 114 & 4 & 0.27 & & & 1.16 \\
\hline 118 & 4 & 0.09 & & & 1.15 \\
\hline 119 & 3 & 0.63 & & & 1.18 \\
\hline 122 & 2 & -1.17 & & & 1.08 \\
\hline 129 & 4 & -0.18 & & & 1.14 \\
\hline 134 & 3 & 0.99 & & & 1.20 \\
\hline 140 & 4 & 0.36 & & 1.17 & \\
\hline 141 & 3 & -0.99 & & & 1.09 \\
\hline 145 & 3 & 0.63 & & & 1.18 \\
\hline 179 & 4 & -0.09 & & 1.14 & \\
\hline 211 & 4 & 0.27 & & & 1.16 \\
\hline 220 & 2 & 1.17 & & 1.21 & \\
\hline 221 & 4 & 0.27 & & & 1.16 \\
\hline 224 & 0 & -2.07 & & 1.03 & \\
\hline
\end{tabular}


Table 12. -Statistical summary of reported data for standard reference water sample $\mathrm{N}-42$ (nonpreserved nutrient)--Continued total P as P (total Phosphorus) $\mathrm{m} \mathbf{g} / \mathbf{L}$

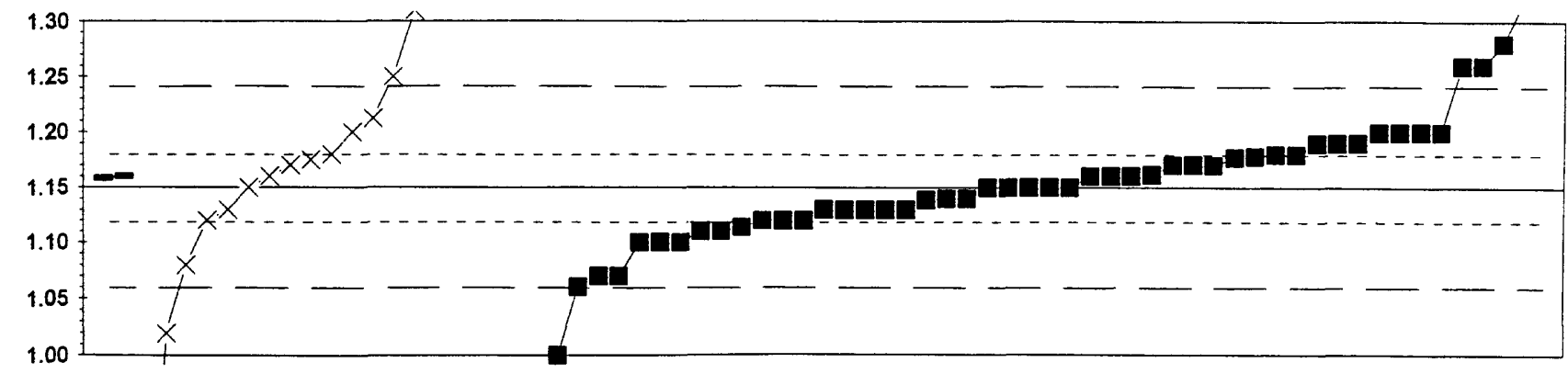

$\longrightarrow 0-x-22-22 \mathrm{ph}$

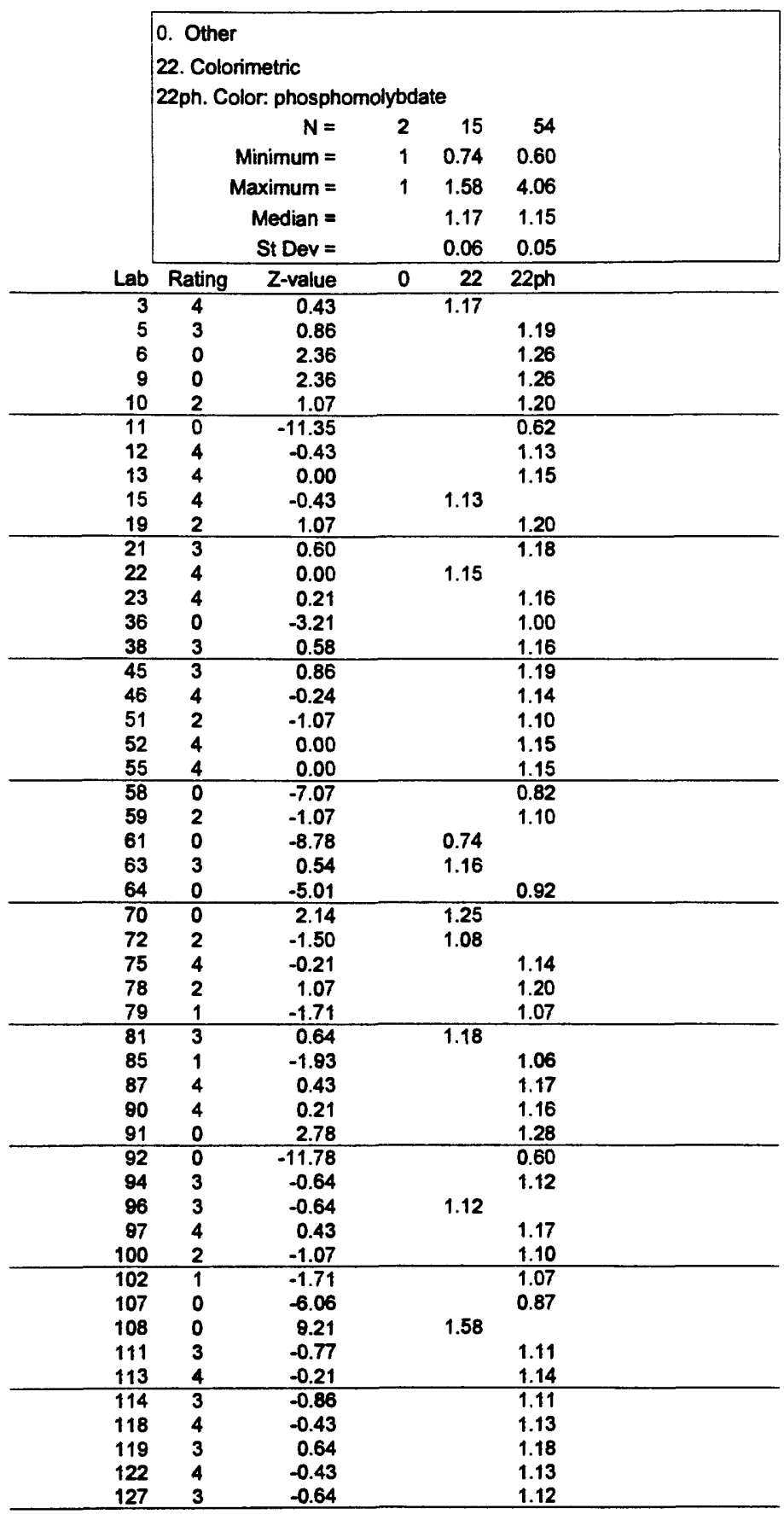

\begin{tabular}{rrrrrr} 
Lab & Rating & Z-value & 0 & 22 & 22 ph \\
\hline 128 & 3 & 0.86 & & & 1.19 \\
129 & 4 & 0.24 & & 1.16 \\
133 & 4 & -0.43 & & & 1.13 \\
134 & 2 & 1.07 & & 1.20 \\
138 & 3 & -0.64 & & 1.12 \\
\hline 142 & 2 & 1.33 & & 1.21 & \\
143 & 4 & -0.43 & & & 1.13 \\
145 & 4 & 0.00 & & & 1.15 \\
158 & 4 & 0.43 & & 1.16 & \\
179 & 4 & 0.21 & & 1.17 \\
180 & 3 & -0.86 & & 1.31 & 1.11 \\
194 & 0 & 3.43 & & & 4.06 \\
203 & 0 & 62.31 & & & 1.16 \\
204 & 4 & 0.21 & & & \\
210 & 4 & 0.17 & 1.16 & & 1.18 \\
211 & 3 & 0.64 & & & \\
213 & 4 & 0.21 & 1.16 & 1.20 & \\
220 & 2 & 1.07 & & 1.02 & \\
224 & 0 & -2.78 & & & 1.32 \\
225 & 0 & 3.64 & & & \\
\hline 227 & 4 & 0.00 & & & 1.15
\end{tabular}


Table 12. -Statistical summary of reported data for standard reference water sample N-42 (preserved nutrient)--Continued PO4 as P (Orthophosphate) $\quad \mathrm{m} \mathrm{g} / \mathrm{L}$

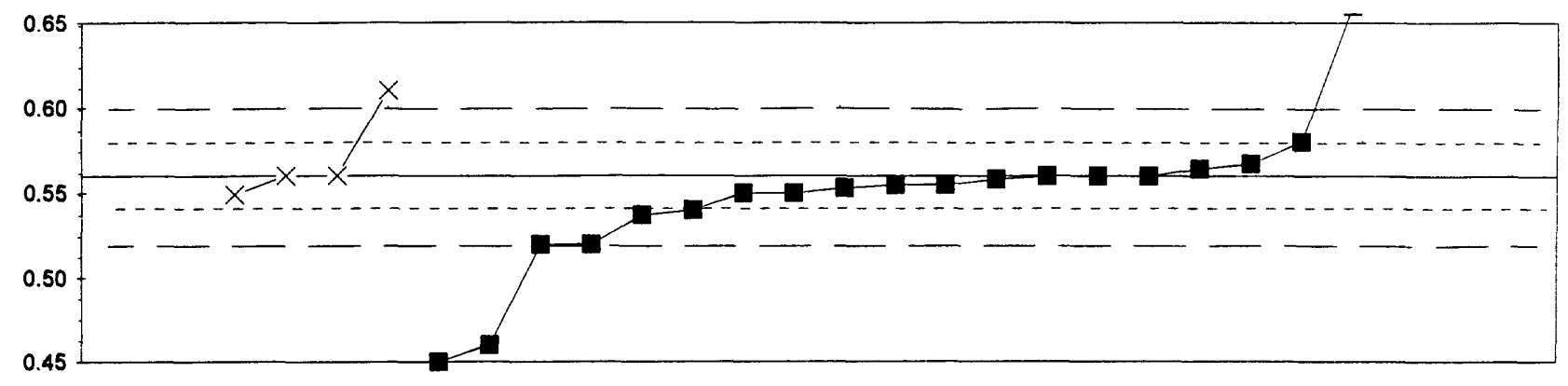

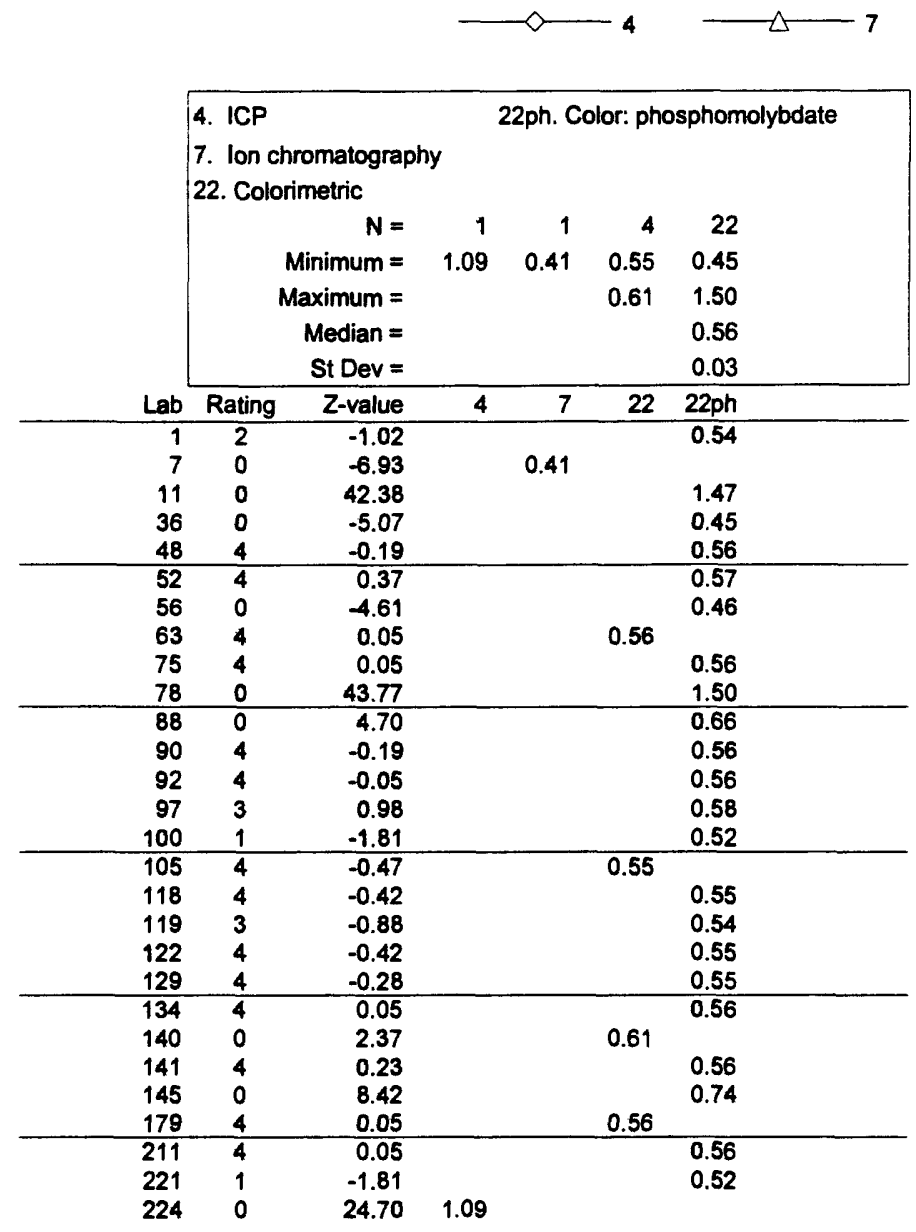


Table 12. -Statistical summary of reported data for standard reference water sample N-42 (nonpreserved nutrient)--Continued PO4 as P (Orthophosphate) $\mathrm{m} \mathrm{g} / \mathrm{L}$

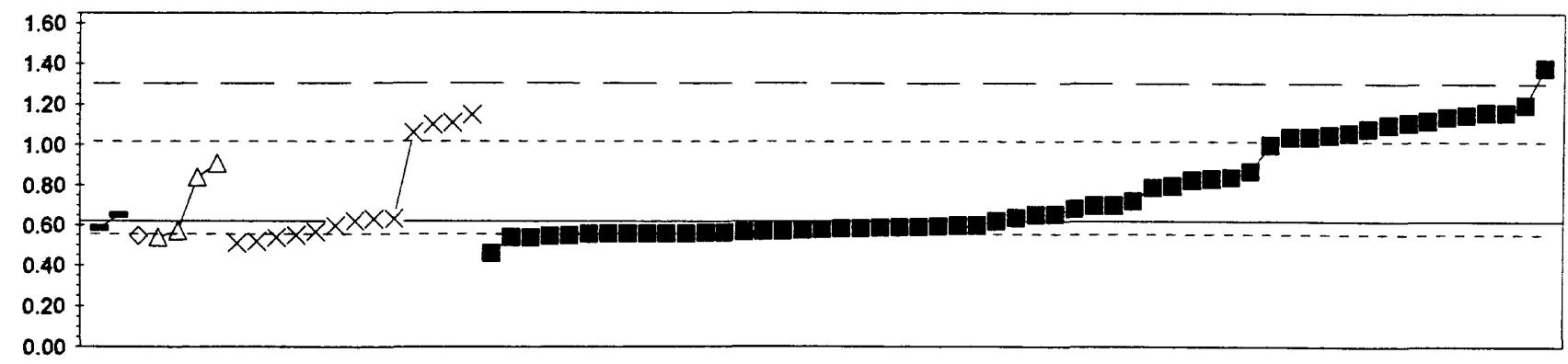

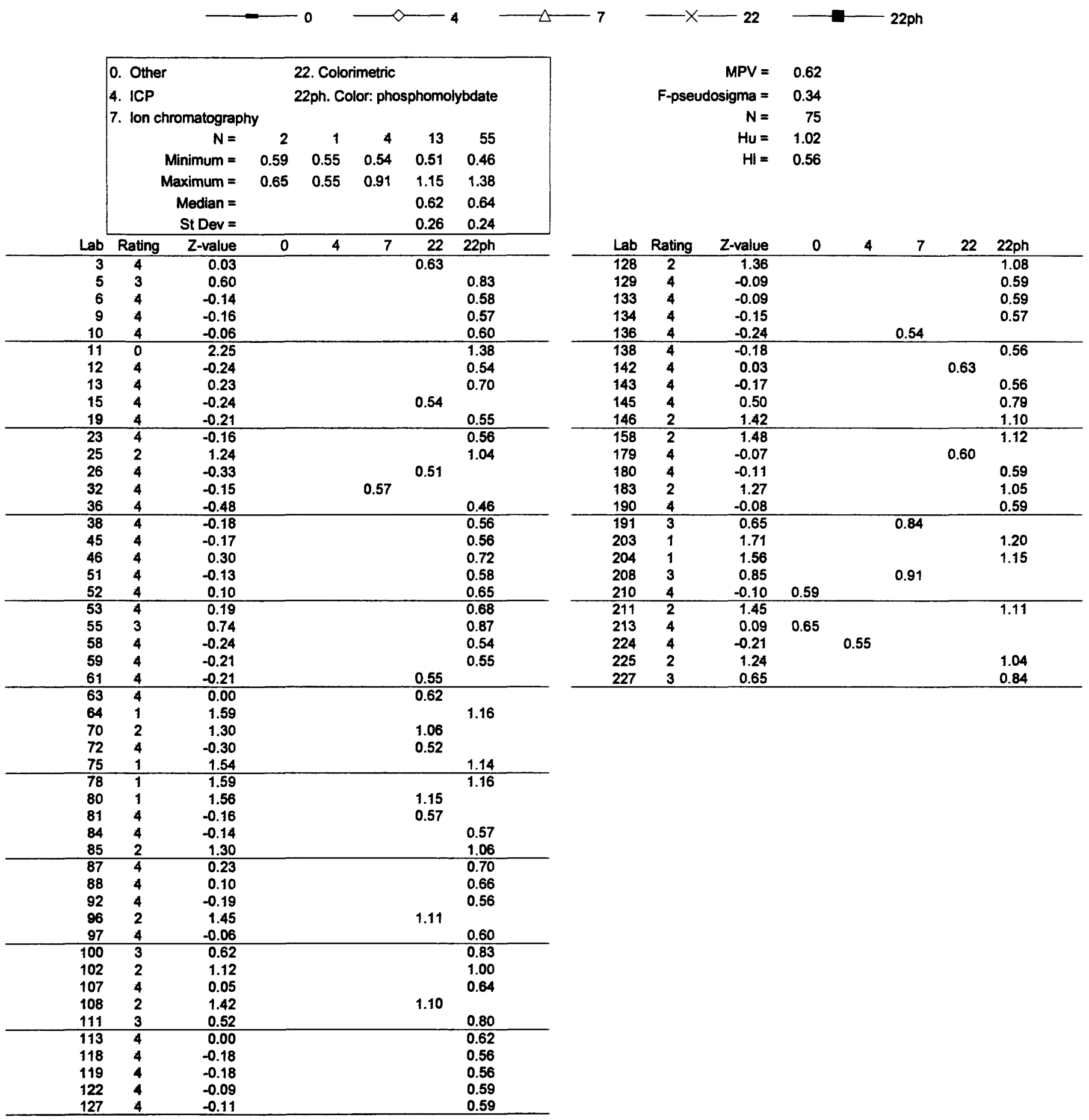


Table 13. -Statistical summary of reported data for standard reference water sample P-22 (low ionic strength)

Definition of analytical methods, abbreviations, and symbols.

Analytical methods

0. Other/Not reported

1. AA: direct air

atomic absorption: direct, air

2. AA: direct $\mathrm{N} 2 \mathrm{O}$

atomic absorption: direct, nitrous oxide

4. ICP

inductively coupled plasma

5. DCP

direct current plasma

6. ICPMS

inductively coupled plasma/mass spectrometry

7. IC

ion chromatography

12. Flame emission

20. Titrate: color

titration: colorimetric [color reagent specified]

21. Titrate: electro

titration: electrometric

22: Color

colorimetric [color reagent specified]

40. Ion selective electrode

41. Electro

electrometric [direct reading instrument]

50. Gravimetric

gravimetric [precipitate specified]

51. Turbidimetric

Abbreviations and symbols

$$
\begin{aligned}
N & =\text { number of samples } \\
\text { Stdev } & =\text { traditional standard deviation } \\
\text { MPV } & =\text { most probable value }
\end{aligned}
$$

F-pseudosigma $=$ nonparametric statistic deviation

$\mathrm{Hu}=$ upper hinge value

$\mathrm{HI}=$ lower hinge value

$\mu \mathrm{S} / \mathrm{cm}=$ microsiemens per centimeter at 25 degrees $\mathrm{C}$

$\mathrm{mg} / \mathrm{L}=$ milligrams per liter

Lab $=$ Laboratory code number

$N R=$ not rated, less than value reported

$$
<=\text { less than }
$$

\begin{tabular}{llr}
\hline Constituent & & pege \\
Acid & Acidity as $\mathrm{CaCO} 3$ & 88 \\
$\mathrm{Ca}$ & Calcium & 89 \\
$\mathrm{Cl}$ & Chloride & 90 \\
$\mathrm{~F}$ & Fluoride & 91 \\
$\mathrm{~K}$ & Potassium & 92 \\
$\mathrm{Mg}$ & Magnesium & 93 \\
$\mathrm{Na}$ & Sodium & 94 \\
$\mathrm{PH}$ & & 95 \\
$\mathrm{PO}$ as P & Orthophosphate & 96 \\
SO4 & Sulfate & 97 \\
$\mathrm{SpCond}$ & Specific Conductance & 98 \\
\hline
\end{tabular}


Table 13. -Statistical summary of reported data for standard reference water sample P-22 (low ionic strength)--Continued Acid as $\mathrm{CaCO} 3$ (Acidity) $\mathrm{m}$ g/L
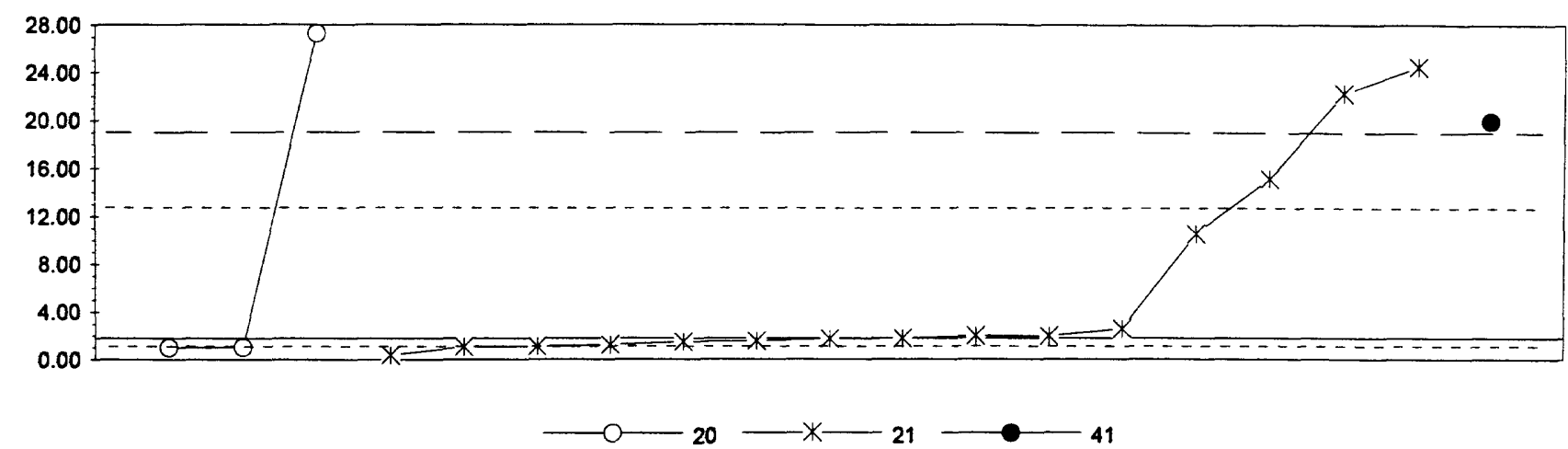

\begin{tabular}{|c|c|c|c|c|c|}
\hline \multirow[b]{2}{*}{ Lab } & $\begin{array}{l}\text { 20. Titra } \\
\text { 21. Titra } \\
\text { 41. Direc }\end{array}$ & $\begin{array}{l}\text { Ite: colorimetr } \\
\text { te: electrome } \\
\text { ct reading } \\
\mathrm{N}= \\
\text { Minimum = } \\
\text { Maximum = } \\
\text { Median = } \\
\text { St Dev = }\end{array}$ & $\begin{array}{l}\text { ic } \\
\text { tric } \\
3 \\
1.00 \\
27.30\end{array}$ & $\begin{array}{r}15 \\
0.40 \\
24.50 \\
1.79 \\
8.17\end{array}$ & $\begin{array}{r}1 \\
20.00\end{array}$ \\
\hline & Rating & Z-value & 20 & 21 & 41 \\
\hline $\begin{array}{r}1 \\
3 \\
11 \\
15 \\
23 \\
\end{array}$ & $\begin{array}{c}4 \\
\text { NR } \\
4 \\
4 \\
4 \\
\end{array}$ & $\begin{array}{r}-0.08 \\
-0.06 \\
0.00 \\
-0.03\end{array}$ & & $\begin{array}{l}1.12 \\
<10 \\
1.26 \\
1.76 \\
1.57 \\
\end{array}$ & \\
\hline $\begin{array}{l}25 \\
36 \\
38 \\
58 \\
61\end{array}$ & $\begin{array}{l}4 \\
4 \\
4 \\
2 \\
4 \\
\end{array}$ & $\begin{array}{r}0.09 \\
-0.08 \\
0.00 \\
1.02 \\
-0.16\end{array}$ & & $\begin{array}{r}2.60 \\
1.10 \\
1.79 \\
10.60 \\
0.40 \\
\end{array}$ & \\
\hline $\begin{array}{r}63 \\
78 \\
94 \\
136 \\
141 \\
\end{array}$ & $\begin{array}{l}4 \\
4 \\
0 \\
1 \\
0\end{array}$ & $\begin{array}{r}-0.09 \\
0.02 \\
2.62 \\
1.55 \\
2.36\end{array}$ & 1.00 & $\begin{array}{r}2.00 \\
24.50 \\
15.20 \\
22.30 \\
\end{array}$ & \\
\hline $\begin{array}{l}145 \\
180 \\
196 \\
210 \\
224\end{array}$ & $\begin{array}{c}4 \\
4 \\
4 \\
N R \\
0\end{array}$ & $\begin{array}{r}0.02 \\
-0.03 \\
-0.09 \\
\\
2.94\end{array}$ & $\begin{array}{r}1.05 \\
27.30\end{array}$ & $\begin{array}{l}2.00 \\
1.50 \\
<2\end{array}$ & \\
\hline 225 & 0 & 2.10 & & & 20.00 \\
\hline
\end{tabular}


Table 13. -Statistical summary of reported data for standard reference water sample P-22 (low ionic strength)--Continued Ca (Calcium)

$\mathrm{m} \mathbf{g} / \mathrm{L}$

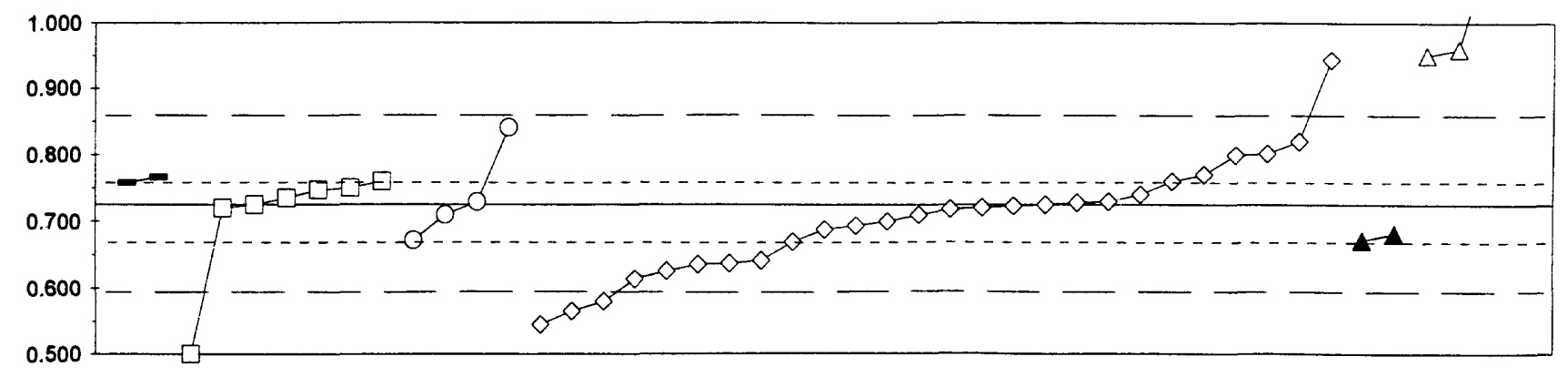

0

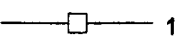

$\longrightarrow \mathrm{O}-2$

\begin{tabular}{|c|c|c|c|c|c|c|c|c|}
\hline \multirow[b]{7}{*}{ Lab } & $\begin{array}{l}\text { 0. Other } \\
\text { 1. AA: di } \\
\text { 2. AA: di }\end{array}$ & direct air & oxide & & $\begin{array}{l}\text { 4. ICP } \\
\text { 5. } D C P \\
\text { 7. IC }\end{array}$ & & & \\
\hline & & $\mathbf{N}=$ & 2 & 7 & 4 & 26 & 2 & 4 \\
\hline & & Minimum $=$ & 0.758 & 0.500 & 0.672 & 0.545 & 0.672 & 0.950 \\
\hline & & Maximum $=$ & 0.767 & 0.760 & 0.840 & 0.943 & 0.682 & 1.148 \\
\hline & & Median = & & 0.735 & & 0.715 & & \\
\hline & & St Dev = & & 0.092 & & 0.087 & & \\
\hline & Rating & Z-value & 0 & 1 & 2 & 4 & 5 & 7 \\
\hline 1 & 4 & 0.08 & & & & 0.730 & & \\
\hline 2 & 0 & 6.48 & & & & & & 1.148 \\
\hline 3 & 0 & -2.22 & & & & 0.580 & & \\
\hline 5 & 4 & -0.09 & & & & 0.719 & & \\
\hline 11 & 3 & 0.69 & & & & 0.770 & & \\
\hline 15 & 2 & 1.18 & & & & 0.802 & & \\
\hline 23 & 3 & 0.64 & 0.767 & & & & & \\
\hline 25 & 2 & -1.36 & & & & 0.636 & & \\
\hline 26 & 0 & 3.60 & & & & & & 0.960 \\
\hline 33 & 3 & -0.66 & & & & & 0.682 & \\
\hline 36 & 3 & -0.81 & & & 0.672 & & & \\
\hline 38 & 4 & 0.08 & & & 0.730 & & & \\
\hline 39 & 3 & -0.57 & & & & 0.688 & & \\
\hline 44 & 4 & 0.15 & & 0.735 & & & & \\
\hline 46 & 4 & -0.03 & & & & 0.723 & & \\
\hline 48 & 2 & 1.46 & & & & 0.820 & & \\
\hline 52 & 4 & -0.06 & & & & 0.721 & & \\
\hline 58 & 0 & -3.45 & & 0.500 & & & & \\
\hline 61 & 1 & -1.52 & & & & 0.626 & & \\
\hline 63 & 0 & 3.34 & & & & 0.943 & & \\
\hline 64 & 3 & -0.84 & & & & 0.670 & & \\
\hline 78 & 4 & 0.38 & & 0.750 & & & & \\
\hline 93 & 2 & -1.33 & & & & 0.638 & & \\
\hline 94 & 4 & 0.00 & & & & 0.725 & & \\
\hline 101 & 4 & 0.34 & & 0.747 & & & & \\
\hline 102 & 4 & -0.23 & & & & 0.710 & & \\
\hline 107 & 4 & -0.08 & & 0.720 & & & & \\
\hline 110 & 3 & 0.54 & & 0.760 & & & & \\
\hline 111 & 4 & -0.23 & & & 0.710 & & & \\
\hline 112 & 3 & -0.81 & & & & & 0.672 & \\
\hline 113 & NR & & & $<1$ & & & & \\
\hline 134 & 3 & 0.54 & & & & 0.760 & & \\
\hline 136 & 1 & 1.76 & & & 0.840 & & & \\
\hline 138 & 4 & 0.23 & & & & 0.740 & & \\
\hline 140 & 4 & 0.00 & & 0.725 & & & & \\
\hline 141 & 1 & -1.70 & & & & 0.614 & & \\
\hline 145 & 4 & -0.38 & & & & 0.700 & & \\
\hline 158 & 0 & 3.45 & & & & & & 0.950 \\
\hline 180 & 2 & -1.27 & & & & 0.642 & & \\
\hline 190 & 0 & 6.06 & & & & & & 1.120 \\
\hline 194 & NR & & & & & $<5$ & & \\
\hline 196 & 3 & 0.51 & 0.758 & & & & & \\
\hline 204 & 0 & -2.45 & & & & 0.565 & & \\
\hline 209 & 4 & -0.48 & & & & 0.694 & & \\
\hline 210 & 0 & -2.76 & & & & 0.545 & & \\
\hline $\begin{array}{l}224 \\
225\end{array}$ & $\begin{array}{l}4 \\
2\end{array}$ & $\begin{array}{l}0.05 \\
1.15\end{array}$ & & & & $\begin{array}{l}0.728 \\
0.800\end{array}$ & & \\
\hline
\end{tabular}

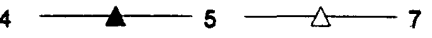

$M P V=0.725$

F-pseudosigma $=0.065$

$N=\quad 45$

$H u=0.760$

$H I=0.672$ 
Table 13. -Statistical summary of reported data for standard reference water sample P-22 (low ionic strength)--Continued Cl (Chloride) $\mathrm{m} \mathrm{g} / \mathbf{L}$

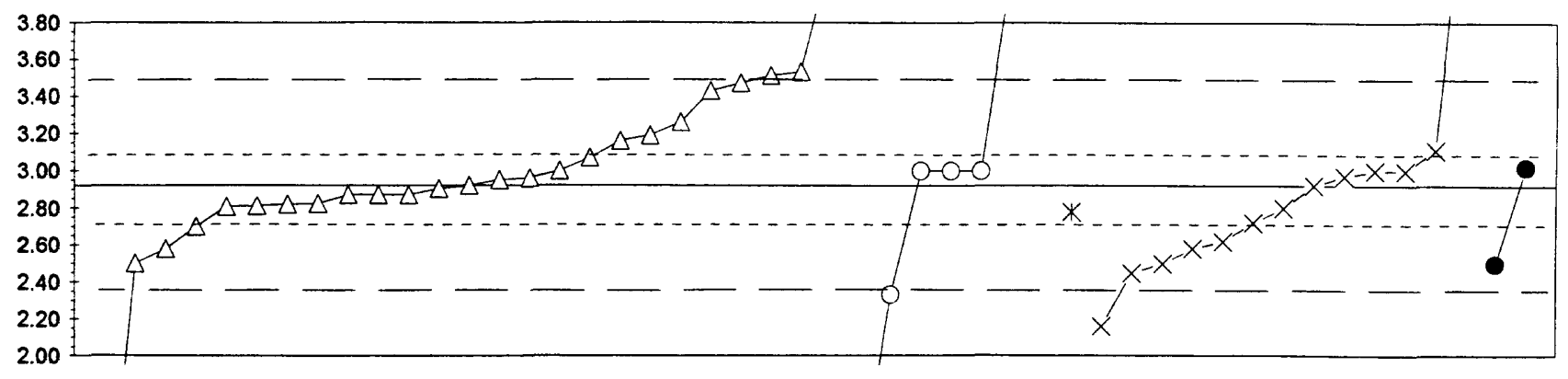

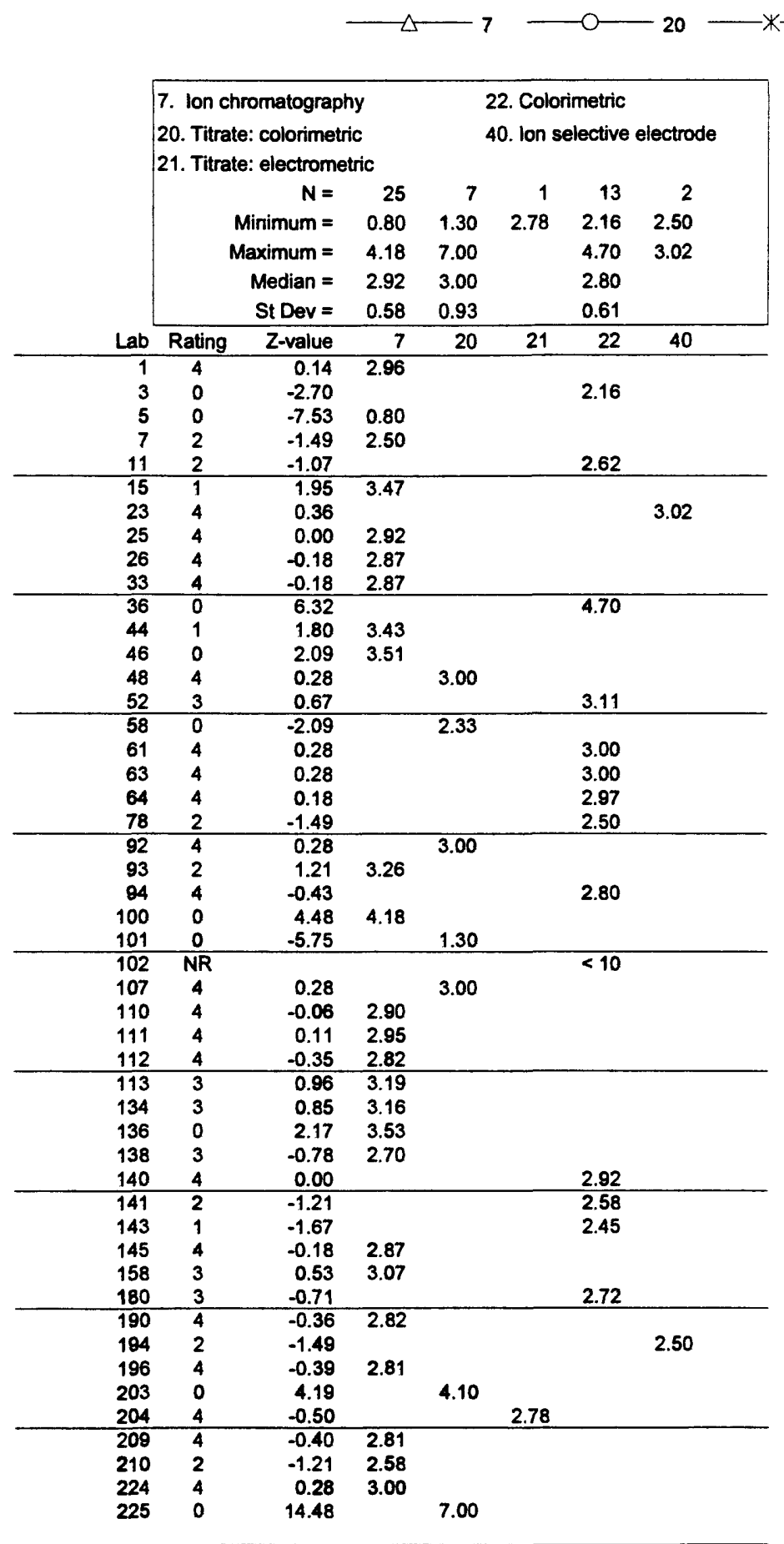


Table 13. -Statistical summary of reported data for standard reference water sample P-22 (low ionic strength)--Continued F (Fluoride) $\mathrm{m}$ g/L

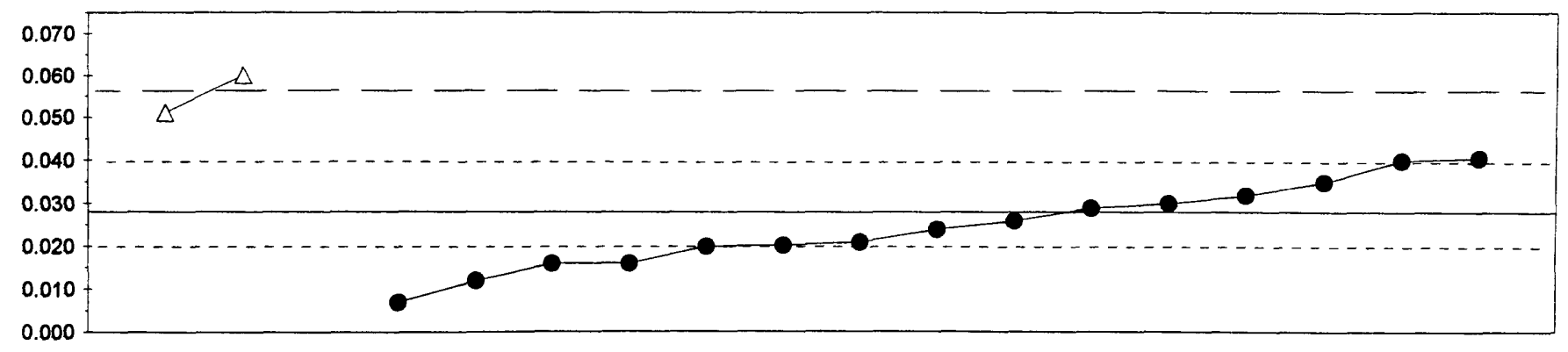

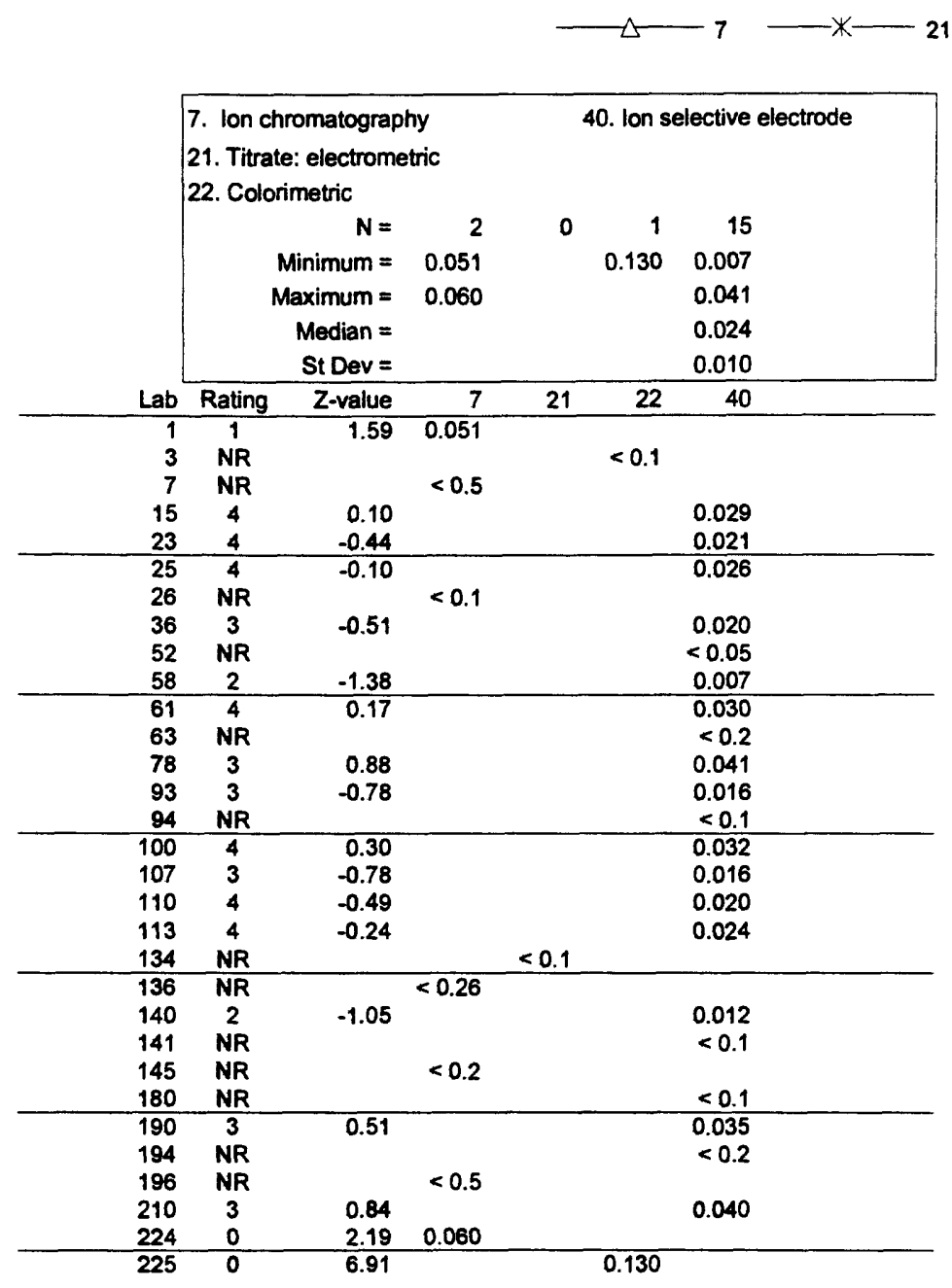


Table 13. -Statistical summary of reported data for standard reference water sample P-22 (low ionic strength)--Continued K (Potassium) $\mathrm{m} \mathrm{g} / \mathrm{L}$

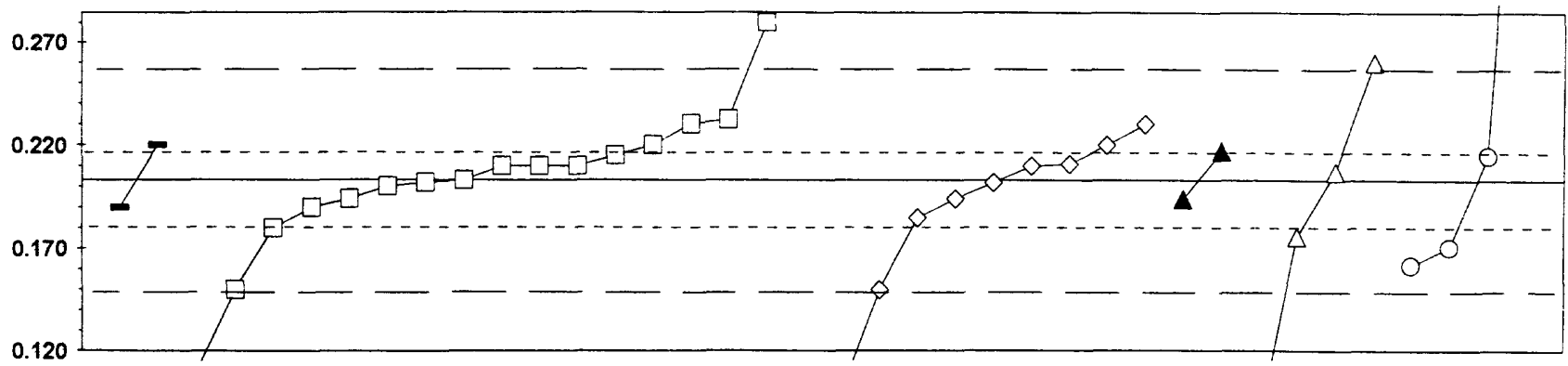

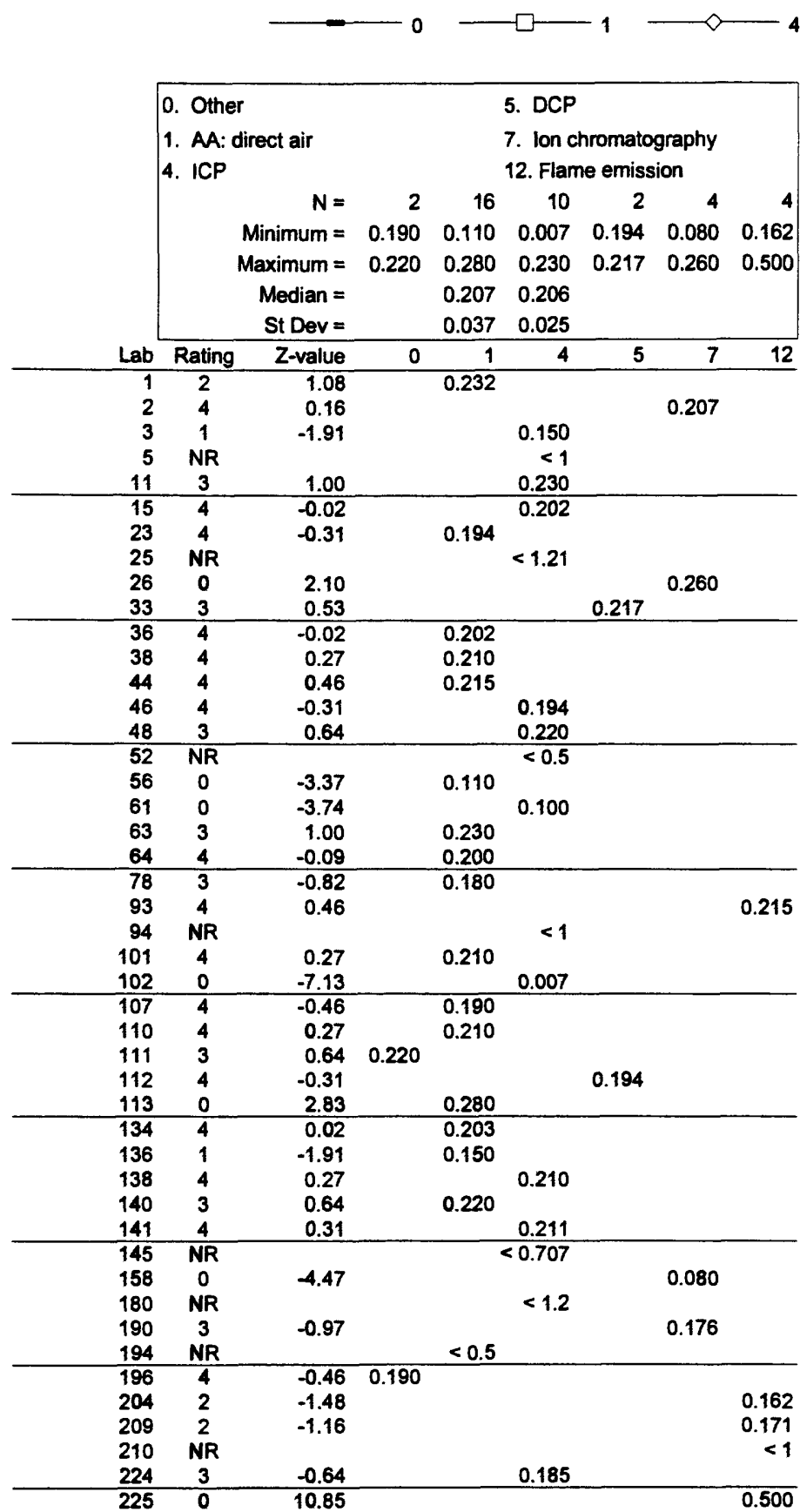


Table 13. -Statistical summary of reported data for standard reference water sample P-22 (low ionic strength)--Continued Mg (Magnesium)

$\mathbf{m} \mathbf{g} / \mathbf{L}$

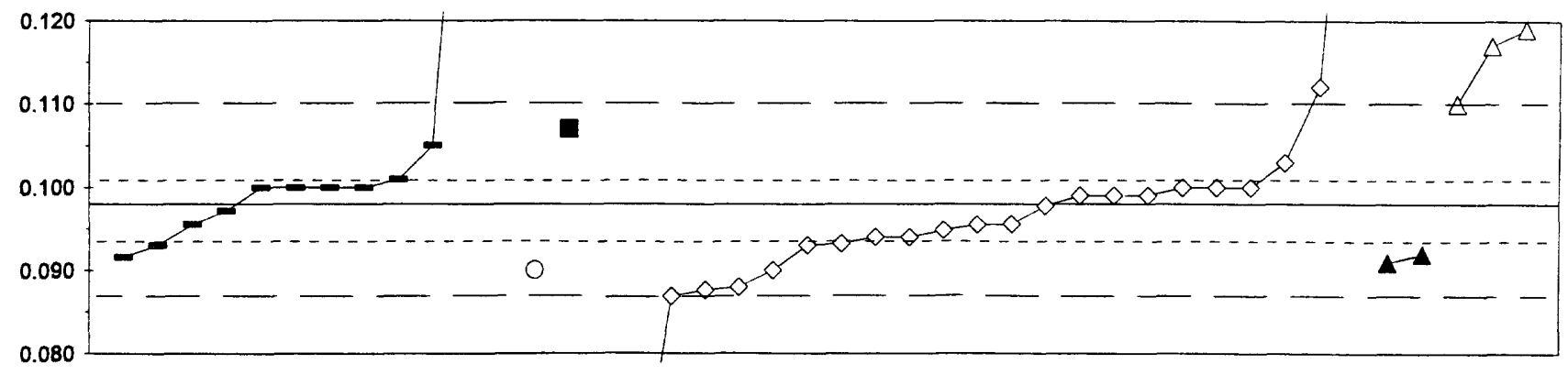

\begin{tabular}{|c|c|c|c|c|c|c|c|c|}
\hline \multirow[b]{7}{*}{ Lab } & \multicolumn{3}{|c|}{$\begin{array}{l}\text { 1. AA: direct air } \\
\text { 2. AA: direct nitrous oxide } \\
\text { 3. AA: graphite fumace }\end{array}$} & \multicolumn{3}{|c|}{$\begin{array}{l}\text { 4. ICP } \\
\text { 5. DCP } \\
\text { 7. IC }\end{array}$} & \multirow{6}{*}{$\begin{array}{r}2 \\
0.091 \\
0.092\end{array}$} & \multirow{6}{*}{$\begin{array}{r}3 \\
0.110 \\
0.119\end{array}$} \\
\hline & & $N=$ & 12 & 1 & 1 & 23 & & \\
\hline & & Minimum = & 0.092 & 0.090 & 0.107 & 0.060 & & \\
\hline & & Maximum = & 0.168 & & & 0.163 & & \\
\hline & & Median = & 0.100 & & & 0.096 & & \\
\hline & & St Dev = & 0.004 & & & 0.006 & & \\
\hline & Rating & Z-value & 1 & 2 & 3 & 4 & 5 & 7 \\
\hline 1 & 4 & 0.10 & & & & 0.099 & & \\
\hline 2 & 0 & 3.14 & & & & & & 0.117 \\
\hline 3 & 3 & -0.74 & & & & 0.094 & & \\
\hline 5 & 4 & 0.27 & & & & 0.100 & & \\
\hline 11 & 4 & 0.27 & & & & 0.100 & & \\
\hline 15 & 4 & -0.10 & & & & 0.098 & & \\
\hline 23 & 4 & -0.49 & 0.096 & & & & & \\
\hline 25 & 1 & -1.75 & & & & 0.088 & & \\
\hline 26 & 1 & 1.96 & & & & & & 0.110 \\
\hline 33 & 2 & -1.08 & & & & & 0.092 & \\
\hline 36 & 0 & 10.39 & 0.160 & & & & & \\
\hline 38 & 4 & 0.44 & 0.101 & & & & & \\
\hline 39 & 4 & 0.10 & & & & 0.099 & & \\
\hline 44 & 4 & -0.20 & 0.097 & & & & & \\
\hline 46 & 4 & -0.49 & & & & 0.096 & & \\
\hline 48 & 0 & -6.48 & & & & 0.060 & & \\
\hline 52 & 0 & 2.29 & & & & 0.112 & & \\
\hline 58 & 3 & -0.91 & 0.093 & & & & & \\
\hline 61 & 3 & -0.88 & & & & 0.093 & & \\
\hline 63 & 0 & 10.89 & & & & 0.163 & & \\
\hline 64 & 4 & 0.27 & 0.100 & & & & & \\
\hline 78 & 4 & 0.27 & 0.100 & & & & & \\
\hline 93 & 0 & -6.48 & & & & 0.060 & & \\
\hline 94 & 3 & 0.78 & & & & 0.103 & & \\
\hline 101 & 2 & 1.11 & 0.105 & & & & & \\
\hline 102 & 1 & -1.92 & & & & 0.087 & & \\
\hline 107 & 4 & 0.27 & 0.100 & & & & & \\
\hline 110 & 4 & 0.27 & 0.100 & & & & & \\
\hline 111 & 2 & -1.42 & & 0.090 & & & & \\
\hline 112 & 2 & -1.25 & & & & & 0.091 & \\
\hline 113 & NR & & $<0.2$ & & & & & \\
\hline 134 & 2 & -1.42 & & & & 0.090 & & \\
\hline 136 & 0 & 11.74 & 0.168 & & & & & \\
\hline 138 & 4 & 0.10 & & & & 0.099 & & \\
\hline 140 & 2 & -1.15 & 0.092 & & & & & \\
\hline 141 & 3 & -0.74 & & & & 0.094 & & \\
\hline 145 & NR & & & & & $<0.19$ & & \\
\hline 180 & 3 & -0.91 & & & & 0.093 & & \\
\hline 190 & 0 & 3.47 & & & & & & 0.119 \\
\hline 194 & NR & & & & & $<1$ & & \\
\hline 196 & 2 & 1.45 & & & 0.107 & & & \\
\hline 204 & 4 & 0.27 & & & & 0.100 & & \\
\hline 209 & 4 & -0.47 & & & & 0.096 & & \\
\hline 210 & 3 & -0.61 & & & & 0.095 & & \\
\hline 224 & 1 & -1.80 & & & & 0.088 & & \\
\hline
\end{tabular}


Table 13. -Statistical summary of reported data for standard reference water sample P-22 (low ionic strength)--Continued $\mathrm{Na}$ (Sodium) $\mathrm{m} \mathrm{g} / \mathrm{L}$

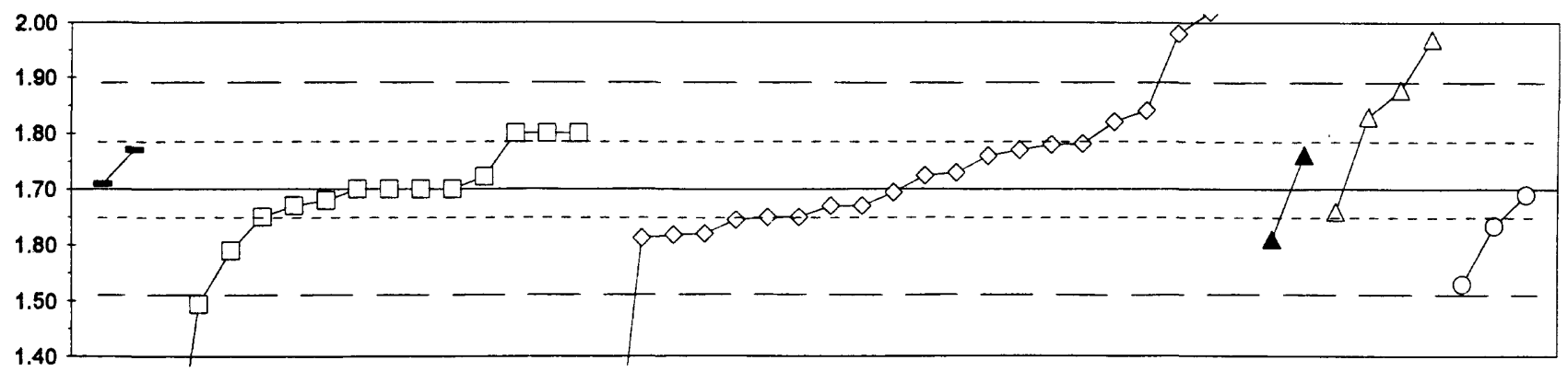

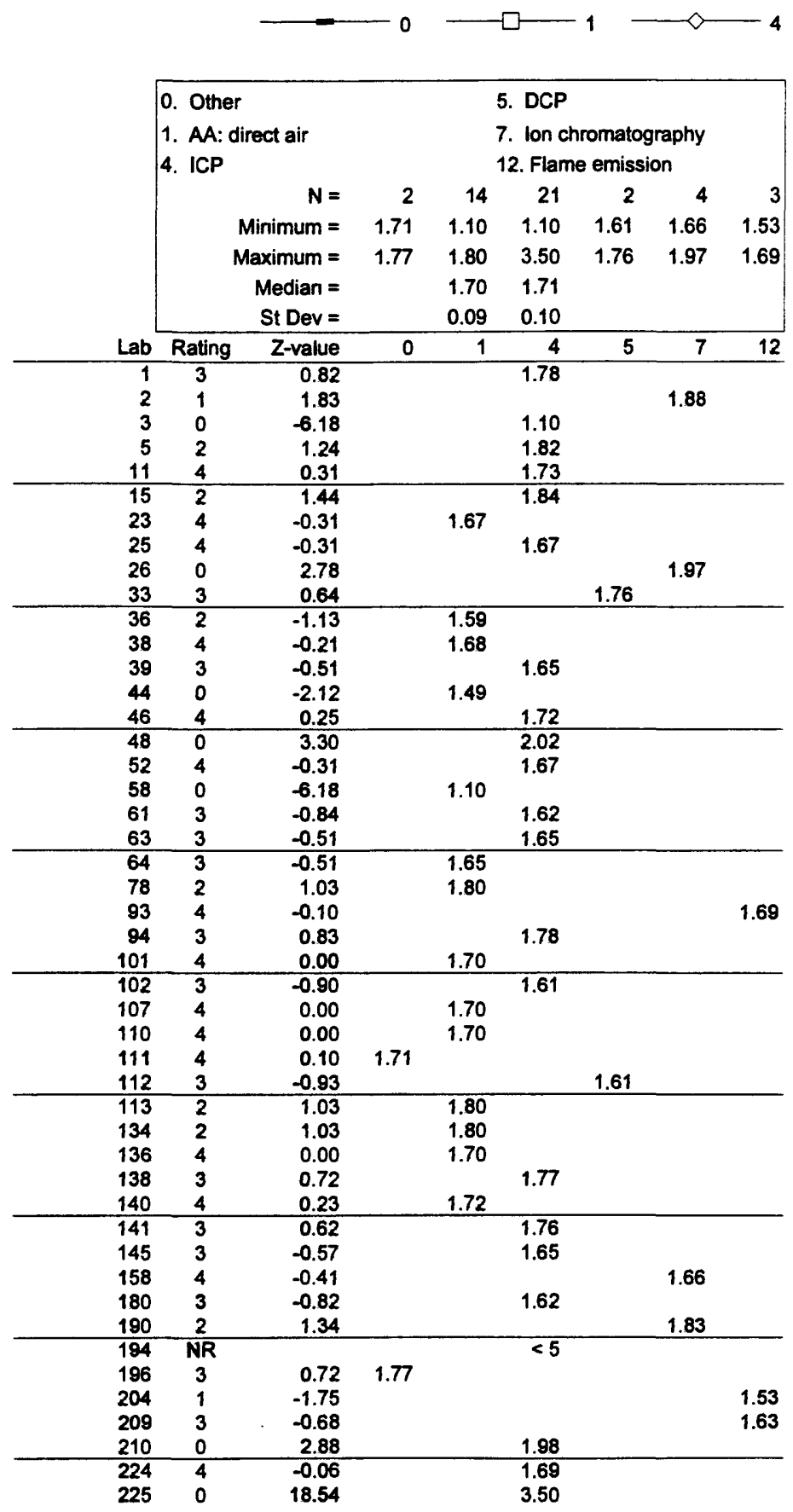


Table 13. -Statistical summary of reported data for standard reference water sample P-22 (low ionic strength)-Continued pH

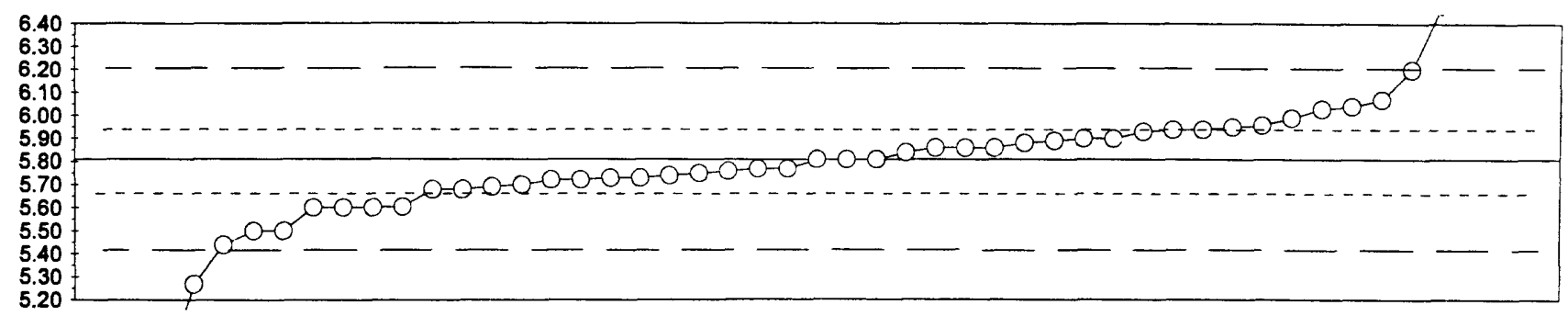

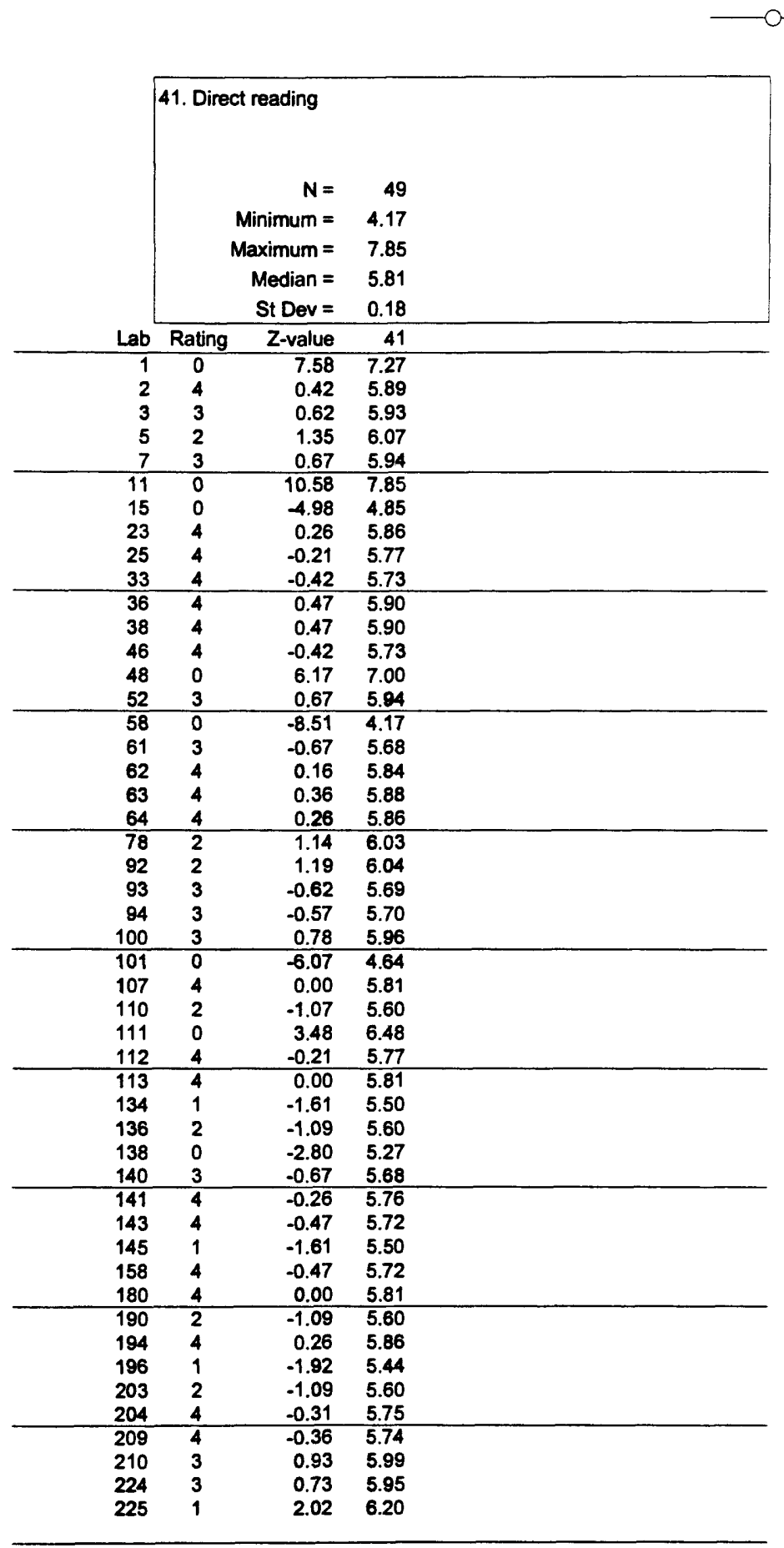


Table 13. -Statistical summary of reported data for standard reference water sample P-22 (low ionic strength)--Continued PO4 as P (Orthophosphate) $\quad \mathrm{m} \mathrm{g} / \mathrm{L}$

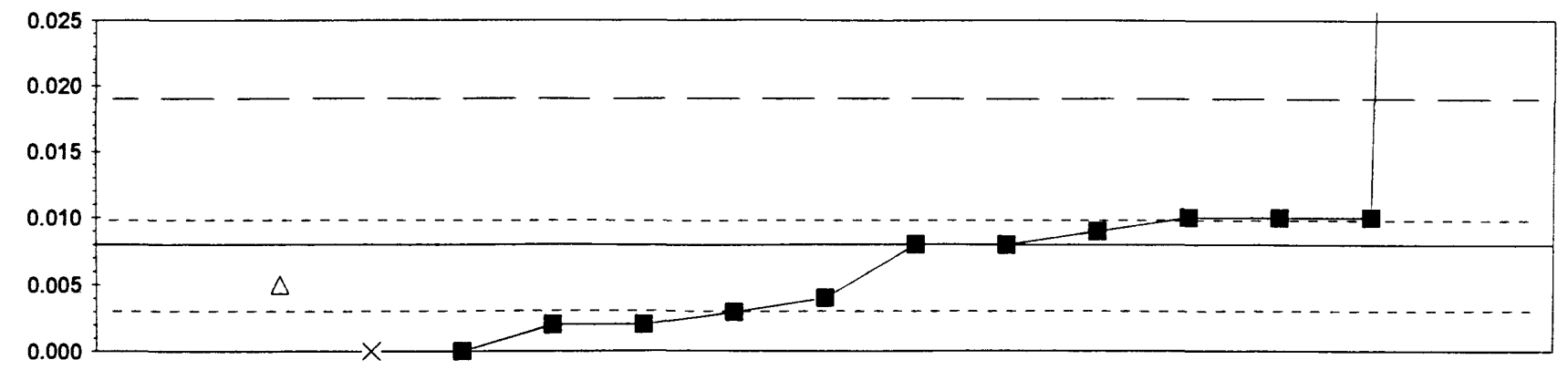

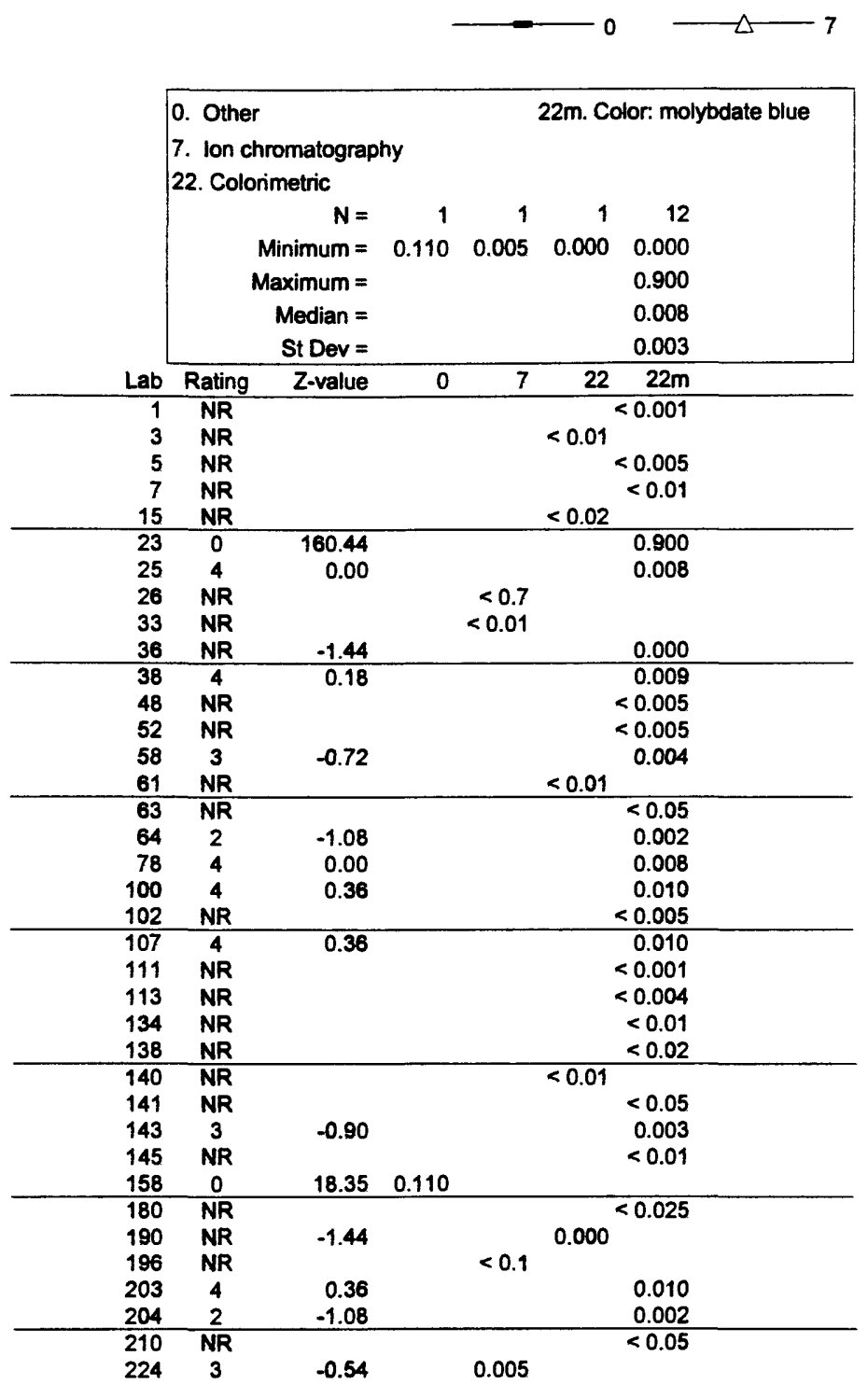


Table 13. -Statistical summary of reported data for standard reference water sample P-22 (low ionic strength)-Continued SO4 (Sulfate) m g/l

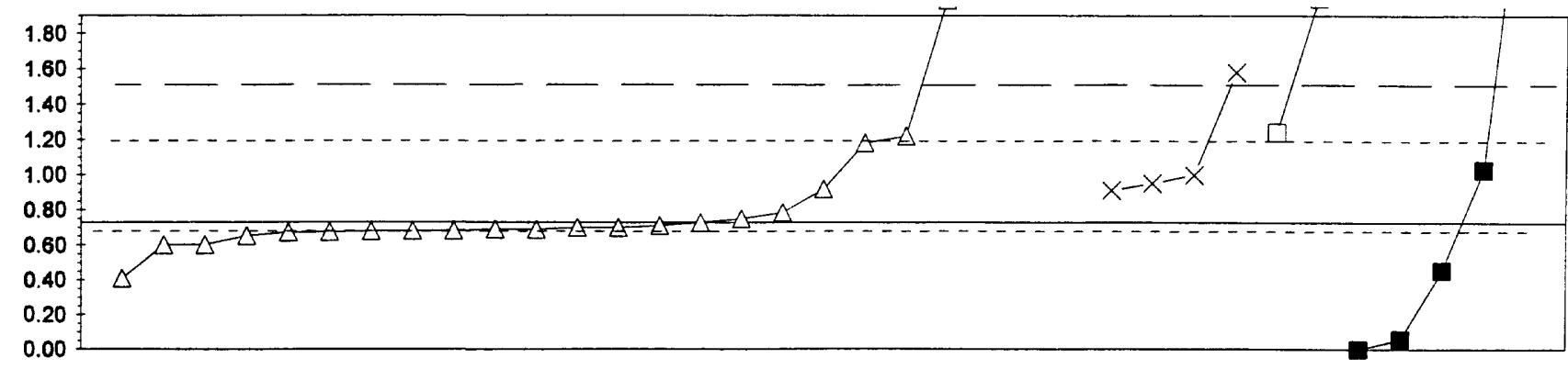

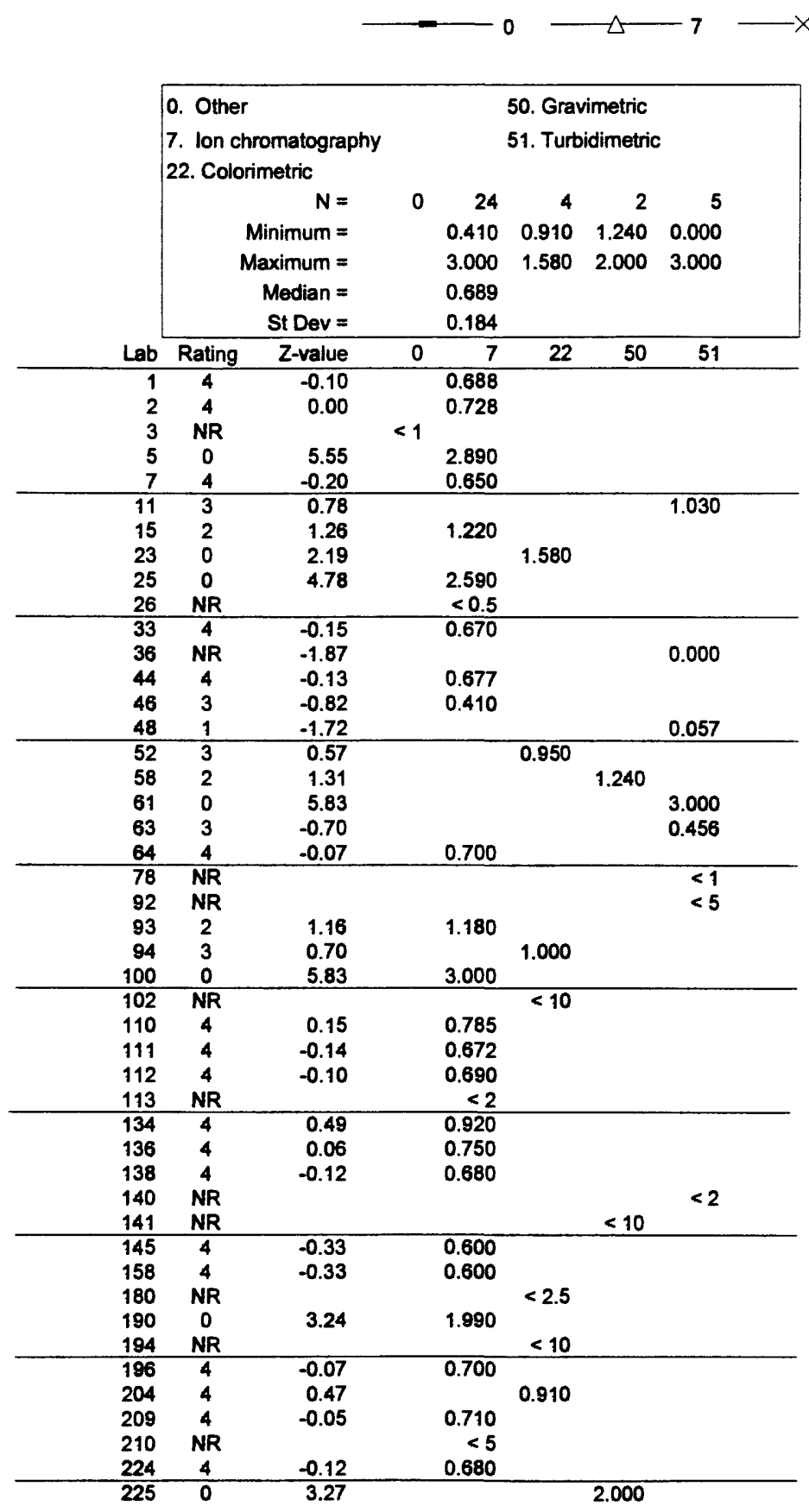


Table 13. -Statistical summary of reported data for standard reference water sample P-22 (low ionic strength)-Continued Sp Cond (Specific Conductance)
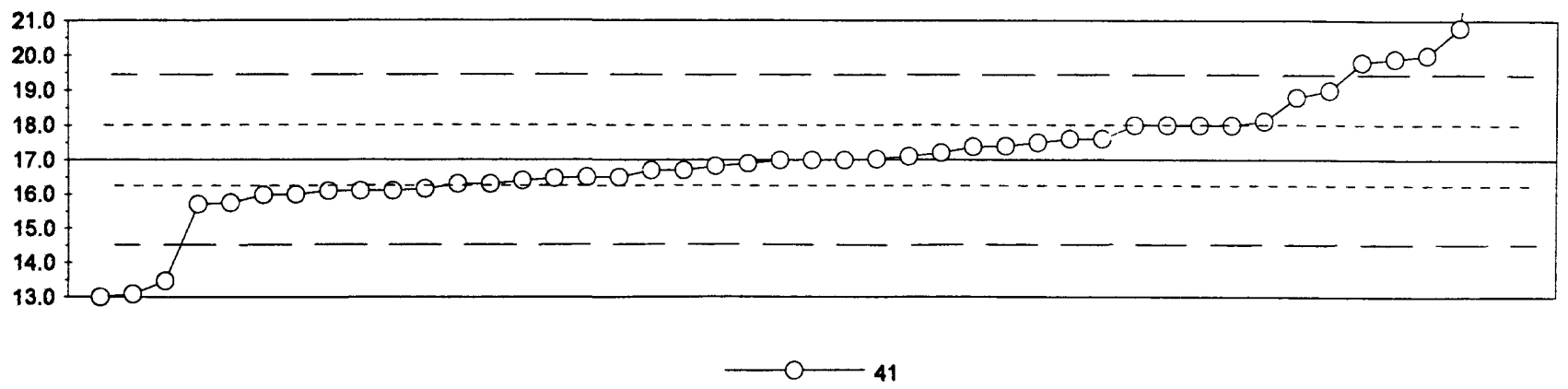

\begin{tabular}{|c|c|c|c|}
\hline \multirow[b]{3}{*}{ Lab } & \multicolumn{3}{|c|}{ 41. Direct reading } \\
\hline & 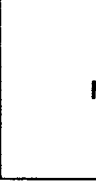 & $\begin{array}{r}\mathrm{N}= \\
\text { Minimum }= \\
\text { Maximum }= \\
\text { Median = } \\
\text { St Dev }=\end{array}$ & $\begin{array}{r}45 \\
13.0 \\
33.5 \\
17.0 \\
1.3 \\
\end{array}$ \\
\hline & Rating & Z-value & 41 \\
\hline $\begin{array}{l}1 \\
2 \\
3 \\
5 \\
7\end{array}$ & $\begin{array}{l}4 \\
0 \\
4 \\
0 \\
3\end{array}$ & $\begin{array}{r}0.40 \\
-2.80 \\
-0.24 \\
2.30 \\
-0.71\end{array}$ & $\begin{array}{l}17.5 \\
13.5 \\
16.7 \\
19.9 \\
16.1\end{array}$ \\
\hline $\begin{array}{l}11 \\
15 \\
23 \\
25 \\
33 \\
\end{array}$ & $\begin{array}{l}3 \\
2 \\
3 \\
1 \\
4\end{array}$ & $\begin{array}{r}-0.56 \\
1.43 \\
-0.56 \\
1.59 \\
-0.40 \\
\end{array}$ & $\begin{array}{l}16.3 \\
18.8 \\
16.3 \\
19.0 \\
16.5 \\
\end{array}$ \\
\hline $\begin{array}{l}36 \\
38 \\
46 \\
48 \\
52\end{array}$ & $\begin{array}{l}0 \\
4 \\
4 \\
4 \\
4\end{array}$ & $\begin{array}{r}2.22 \\
0.16 \\
0.08 \\
-0.43 \\
-0.48 \\
\end{array}$ & $\begin{array}{l}19.8 \\
17.2 \\
17.1 \\
16.5 \\
16.4\end{array}$ \\
\hline $\begin{array}{l}58 \\
61 \\
62 \\
63 \\
64\end{array}$ & $\begin{array}{l}4 \\
4 \\
3 \\
2 \\
4\end{array}$ & $\begin{array}{r}-0.24 \\
0.48 \\
-0.81 \\
-1.02 \\
0.02 \\
\end{array}$ & $\begin{array}{l}16.7 \\
17.6 \\
16.0 \\
15.7 \\
17.0\end{array}$ \\
\hline $\begin{array}{r}78 \\
93 \\
94 \\
100 \\
101 \\
\end{array}$ & $\begin{array}{l}0 \\
3 \\
4 \\
3 \\
4\end{array}$ & $\begin{array}{r}8.73 \\
-0.99 \\
-0.14 \\
0.87 \\
0.00 \\
\end{array}$ & $\begin{array}{l}28.0 \\
15.8 \\
16.8 \\
18.1 \\
17.0 \\
\end{array}$ \\
\hline $\begin{array}{l}102 \\
107 \\
110 \\
111 \\
113\end{array}$ & $\begin{array}{l}0 \\
4 \\
4 \\
3 \\
4\end{array}$ & $\begin{array}{r}-3.17 \\
-0.08 \\
0.00 \\
-0.71 \\
0.32 \\
\end{array}$ & $\begin{array}{l}13.0 \\
16.9 \\
17.0 \\
16.1 \\
17.4 \\
\end{array}$ \\
\hline $\begin{array}{l}134 \\
136 \\
140 \\
141 \\
143\end{array}$ & $\begin{array}{l}4 \\
3 \\
0 \\
0 \\
4\end{array}$ & $\begin{array}{r}0.48 \\
0.79 \\
-3.09 \\
13.09 \\
-0.40 \\
\end{array}$ & $\begin{array}{l}17.6 \\
18.0 \\
13.1 \\
33.5 \\
16.5\end{array}$ \\
\hline $\begin{array}{l}145 \\
158 \\
180 \\
190 \\
194\end{array}$ & $\begin{array}{l}3 \\
4 \\
0 \\
3 \\
3\end{array}$ & $\begin{array}{r}0.79 \\
0.30 \\
2.38 \\
0.79 \\
-0.79 \\
\end{array}$ & $\begin{array}{l}18.0 \\
17.4 \\
20.0 \\
18.0 \\
16.0 \\
\end{array}$ \\
\hline $\begin{array}{l}196 \\
203 \\
210 \\
224 \\
225 \\
\end{array}$ & $\begin{array}{l}0 \\
3 \\
3 \\
3 \\
4\end{array}$ & $\begin{array}{r}3.02 \\
-0.71 \\
-0.67 \\
0.79 \\
0.00 \\
\end{array}$ & $\begin{array}{l}20.8 \\
16.1 \\
16.2 \\
18.0 \\
17.0 \\
\end{array}$ \\
\hline
\end{tabular}


Table 14. -Statistical summary of reported data for standard reference water sample $\mathrm{Hg}$-18 (mercury)

Definition of analytical methods, abbreviations, and symbols.

Analytical methods

D. Other/Not reported

3. AA: graphite furnace atomic absorption: graphite furnace

11. AA: cold vapor atomic absorption: cold vapor

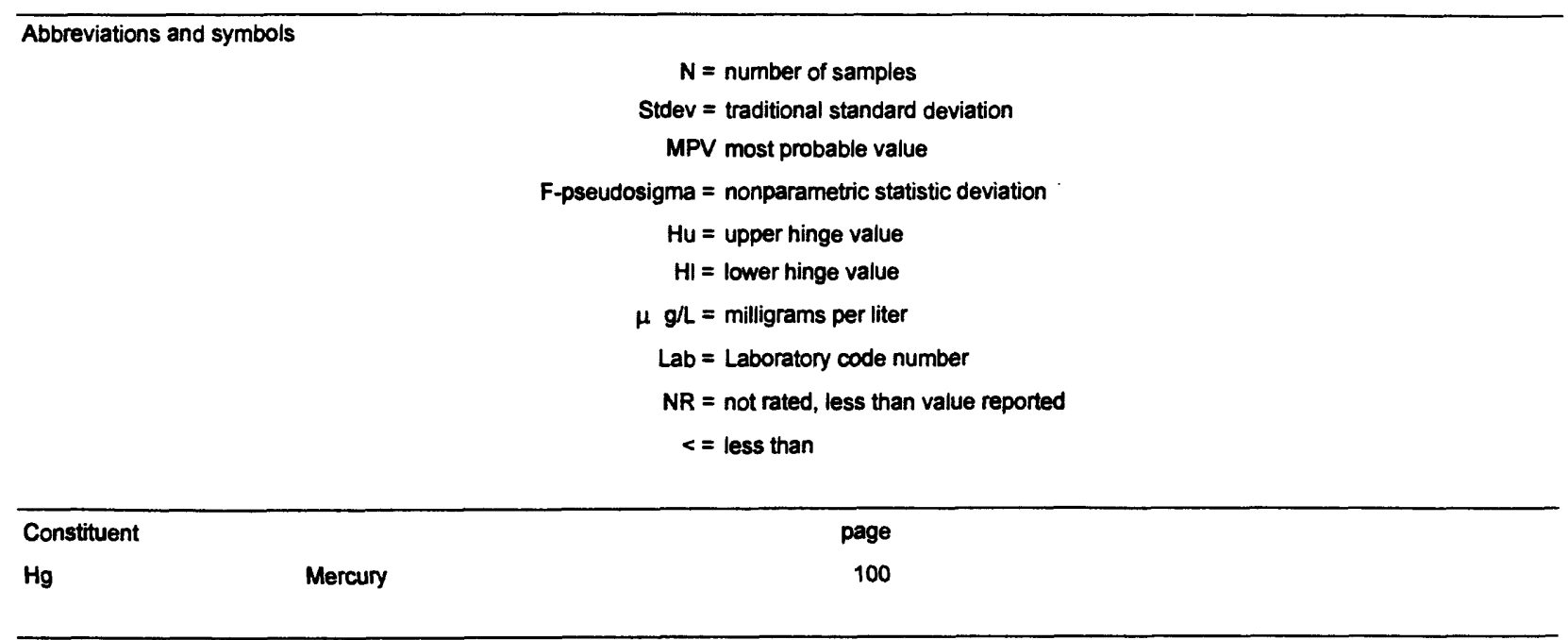


Table 14. -Statistical summary of reported data for standard reference water sample $\mathrm{Hg}-18$ (mercury)-Continued $\mathrm{Hg}$ (Mercury) $\mu \mathrm{g} / \mathrm{L}$
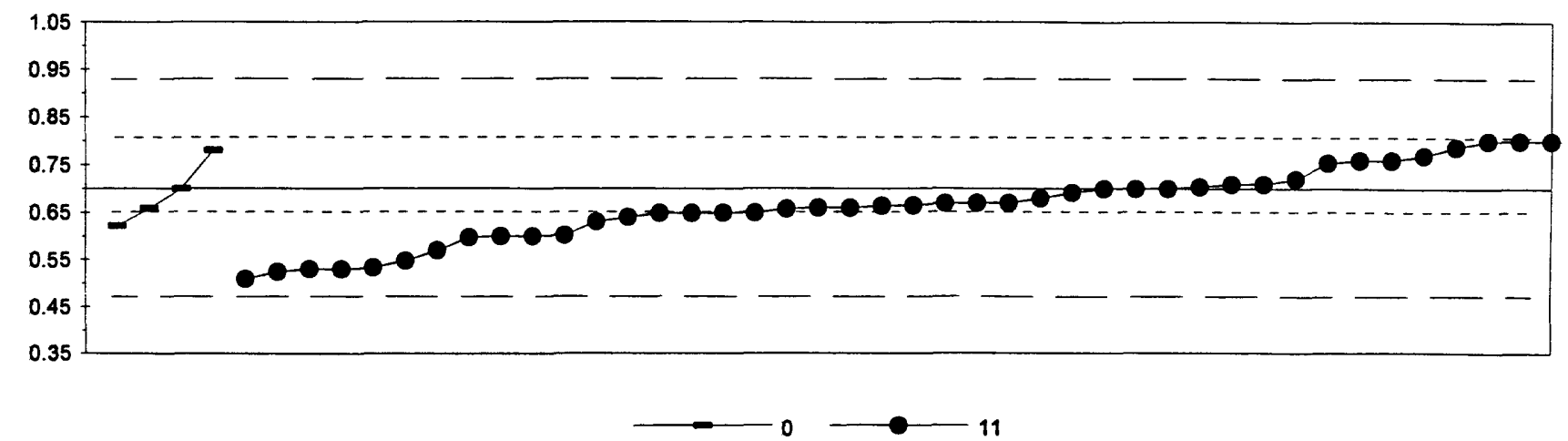

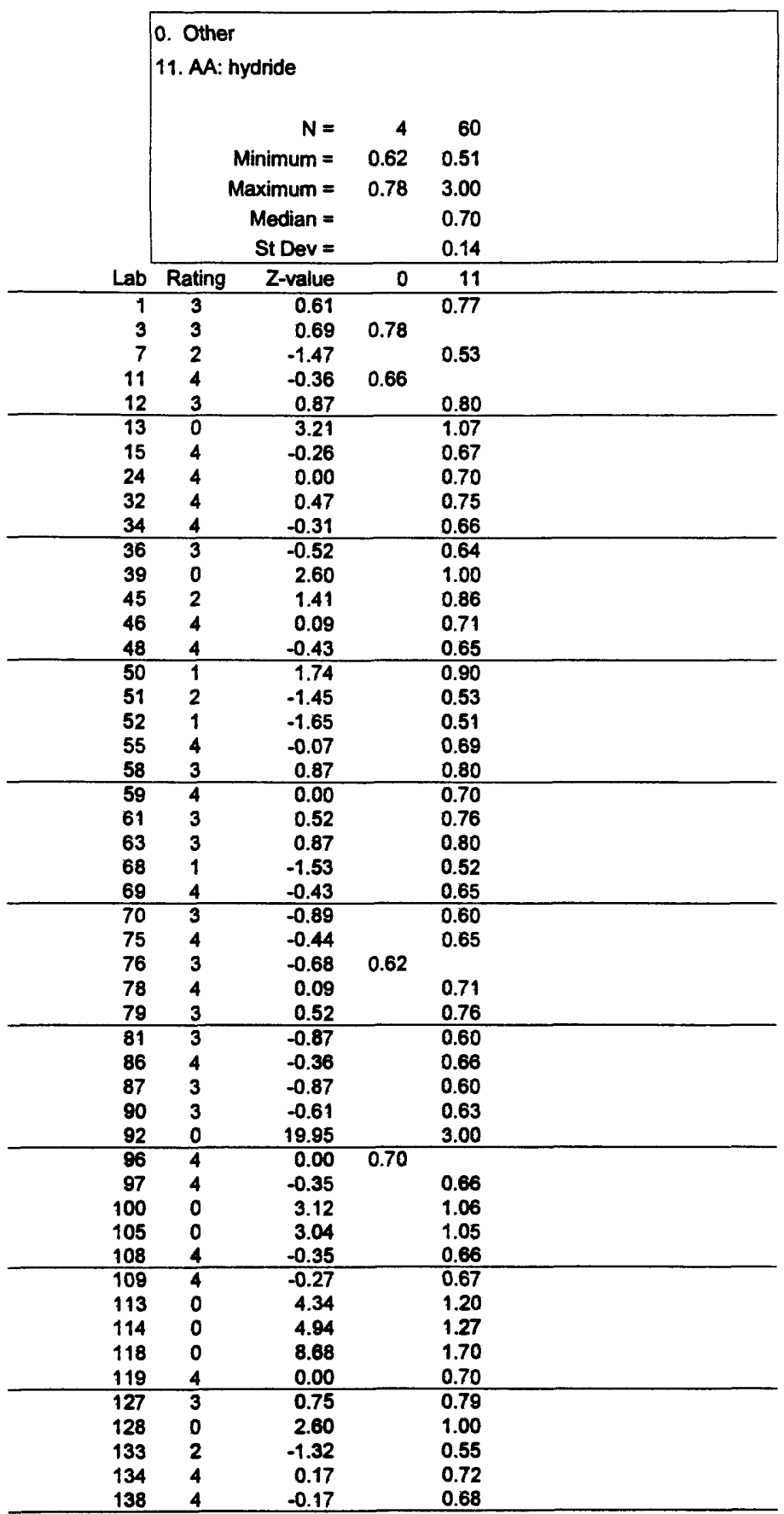

$\begin{array}{rr}\text { MPV } & =0.70 \\ \text { F-pseudosigma } & =0.12 \\ N= & 64 \\ H u= & 0.81 \\ H I= & 0.65\end{array}$

\begin{tabular}{|c|c|c|c|}
\hline Lab & Rating & Z-value & 11 \\
\hline 141 & 4 & 0.03 & 0.70 \\
\hline 142 & 2 & 1.23 & 0.84 \\
\hline 145 & 2 & -1.47 & 0.53 \\
\hline 146 & 4 & -0.31 & 0.66 \\
\hline 149 & 4 & -0.44 & 0.65 \\
\hline 151 & 4 & -0.26 & 0.67 \\
\hline 180 & 3 & -0.85 & 0.60 \\
\hline 194 & 3 & 0.87 & 0.80 \\
\hline 198 & 2 & 1.13 & 0.83 \\
\hline 204 & 3 & 0.87 & 0.80 \\
\hline 210 & 3 & $\begin{array}{l}0.95 \\
<03\end{array}$ & $\begin{array}{r}0.81 \\
\end{array}$ \\
\hline 220 & 2 & -1.13 & 0.57 \\
\hline 221 & 1 & 1.74 & 0.90 \\
\hline 225 & 0 & 11.28 & 2.00 \\
\hline
\end{tabular}


Table 15. -Most probable values for constituents and properties in standard reference samples distributed in April 1994

[MPV, most probable value; ug/L, microgram per liter; mg/L, milligram per liter; uS/cm, microsiemen per centimeter at 25 degrees Celsius]

T-129 (trace constituents)

$\begin{array}{lrr}\text { Analyte } & \text { MPV } & \text { F-pseudosigma } \\ \text { Ag } & 0.37 \mu \mathrm{g} / \mathrm{L} & 1.40 \\ \mathrm{Al} & 50.0 \mu \mathrm{g} / \mathrm{L} & 11.9 \\ \mathrm{As} & 0.55 \mu \mathrm{g} / \mathrm{L} & 1.14 \\ \mathrm{~B} & 11.6 \mu \mathrm{g} / \mathrm{L} & 2.8 \\ \mathrm{Ba} & 34.0 \mu \mathrm{g} / \mathrm{L} & 1.9 \\ \mathrm{Be} & 0.12 \mu \mathrm{g} / \mathrm{L} & 0.13 \\ \mathrm{Ca} & 21.1 \mathrm{~m} \mathrm{~g} / \mathrm{L} & 1.0 \\ \mathrm{Cd} & 0.34 \mu \mathrm{g} / \mathrm{L} & 0.1 \\ \mathrm{Co} & 0.74 \mu \mathrm{g} / \mathrm{L} & 1.20 \\ \mathrm{Cr} & 0.68 \mu \mathrm{g} / \mathrm{L} & 1.3 \\ \mathrm{Cu} & 2.7 \mu \mathrm{g} / \mathrm{L} & 1.4 \\ \mathrm{Fe} & 10.4 \mu \mathrm{g} / \mathrm{L} & 8.2 \\ \mathrm{~K} & 3.00 \mathrm{~m} \mathrm{~g} / \mathrm{L} & 0.20\end{array}$

\begin{tabular}{lrrr}
\multicolumn{1}{r}{ Analyte } & MPV & F-pseudosigma \\
$\mathrm{Li}$ & $18.0 \mu \mathrm{g} / \mathrm{L}$ & 2.6 \\
$\mathrm{Mg}$ & $5.83 \mathrm{~m} \mathrm{~g} / \mathrm{L}$ & 0.25 \\
$\mathrm{Mn}$ & $25.2 \mu \mathrm{g} / \mathrm{L}$ & 2.2 \\
$\mathrm{Mo}$ & $20.3 \mu \mathrm{g} / \mathrm{L}$ & 2.1 \\
$\mathrm{Na}$ & $35.5 \mathrm{~m} \mathrm{~g} / \mathrm{L}$ & 1.5 \\
$\mathrm{Ni}$ & $1.7 \mu \mathrm{g} / \mathrm{L}$ & 1.7 \\
$\mathrm{~Pb}$ & $1.00 \mu \mathrm{g} / \mathrm{L}$ & 1.37 \\
$\mathrm{Sb}$ & $0.55 \mu \mathrm{g} / \mathrm{L}$ & 0.87 \\
$\mathrm{Se}$ & $1.60 \mu \mathrm{g} / \mathrm{L}$ & 1.59 \\
$\mathrm{SiO}$ & $9.15 \mathrm{mg} / \mathrm{L}$ & 0.83 \\
$\mathrm{Sr}$ & $181 \mu \mathrm{g} / \mathrm{L}$ & 11 \\
$\mathrm{~V}$ & $1.0 \mu \mathrm{g} / \mathrm{L}$ & 2.4 \\
$\mathrm{Zn}$ & $72.0 \mu \mathrm{g} / \mathrm{L}$ & 4.8 \\
\hline
\end{tabular}

M-130 (major constituents)

$\begin{array}{llr}\text { Analyte } & \text { MPV } & \text { F-pseudosigma } \\ \text { Alkalinity } & 60.0 \mathrm{~m} \mathrm{~g} / \mathrm{L} & 1.9 \\ \mathrm{~B} & 8.87 \mu \mathrm{g} / \mathrm{L} & 8.23 \\ \mathrm{Ca} & 21.2 \mathrm{~m} \mathrm{~g} / \mathrm{L} & 1.0 \\ \mathrm{CI} & 21.4 \mathrm{~m} \mathrm{~g} / \mathrm{L} & 1.9 \\ \text { DSRD } & 200 \mathrm{~m} \mathrm{~g} / \mathrm{L} & 13 \\ \mathrm{~F} & 1.23 \mathrm{~m} \mathrm{~g} / \mathrm{L} & 0.09 \\ \mathrm{~K} & 3.00 \mathrm{~m} \mathrm{~g} / \mathrm{L} & 0.23 \\ \mathrm{Mg} & 5.90 \mathrm{~m} \mathrm{~g} / \mathrm{L} & 0.27\end{array}$

N-42 (preserved nutrients)

Analyte
$\mathrm{NH} 3$ as N
$\mathrm{NH} 3+$ Org N as N
$\mathrm{NO3}+\mathrm{NO2}$ as N
total P as P
PO4 as P

MPV F-pseudosigma

N-42 (nonpreserved nutrients)

\begin{tabular}{lcrr}
\multicolumn{1}{r}{ Analyte } & MPV & F-pseudosigma \\
$\mathrm{Na}$ & $35.8 \mathrm{~m} \mathrm{~g} / \mathrm{L}$ & 1.6 \\
total P & $0.085 \mathrm{~m} \mathrm{~g} / \mathrm{L}$ & 0.104 \\
$\mathrm{pH}$ & 8.02 & 0.14 \\
$\mathrm{SiO2}$ & $9.20 \mathrm{~m} \mathrm{~g} / \mathrm{L}$ & 0.55 \\
$\mathrm{SO} 4$ & $58.0 \mathrm{~m} \mathrm{~g} / \mathrm{L}$ & 2.6 \\
$\mathrm{SpCond}$ & $335 \mu \mathrm{S} / \mathrm{cm}$ & 10 \\
$\mathrm{Sr}$ & $180 \mu \mathrm{g} / \mathrm{L}$ & 10 \\
$\mathrm{~V}$ & insufficient data &
\end{tabular}

P-22 (low ionic strength)

\begin{tabular}{lrr} 
Analyte & \multicolumn{1}{c}{ MPV } & F-pseudosigma \\
Acidity & $1.79 \mathrm{~m} \mathrm{~g} / \mathrm{L}$ & 8.68 \\
$\mathrm{Ca}$ & $0.725 \mathrm{~m} \mathrm{~g} / \mathrm{L}$ & 0.065 \\
$\mathrm{Cl}$ & $2.92 \mathrm{~m} \mathrm{~g} / \mathrm{L}$ & 0.28 \\
$\mathrm{~F}$ & $0.028 \mathrm{~m} \mathrm{~g} / \mathrm{L}$ & 0.015 \\
$\mathrm{~K}$ & $0.203 \mathrm{~m} \mathrm{~g} / \mathrm{L}$ & 0.027 \\
$\mathrm{Mg}$ & $0.098 \mathrm{~m} \mathrm{~g} / \mathrm{L}$ & 0.006
\end{tabular}

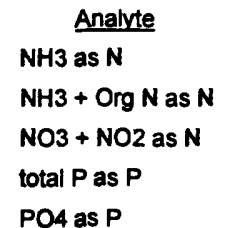

MPV

F-pseudosigma

$0.20 \mathrm{~m} \mathrm{~g} / \mathrm{L} \quad 0.04$

$1.81 \mathrm{~m} \mathrm{~g} / \mathrm{L}$

0.26

$1.92 \mathrm{~m} \mathrm{~g} / \mathrm{L}$

0.07

$1.15 \mathrm{~m} \mathrm{~g} / \mathrm{L}$

0.06

$\mathrm{PO} 4$ as $\mathrm{P}$

$0.25 \mathrm{~m} \mathrm{~g} / \mathrm{L}$

0.44

$1.70 \mathrm{~m} \mathrm{~g} / \mathrm{L} \quad 0.24$

$1.93 \mathrm{~m} \mathrm{~g} / \mathrm{L} \quad 0.08$

$1.15 \mathrm{~m} \mathrm{~g} / \mathrm{L} \quad 0.05$

0.02

$0.62 \mathrm{~m} \mathrm{~g} / \mathrm{L}$

0.34

\begin{tabular}{rlrr} 
& Analyte & MPV & F-pseudosigma \\
8.68 & $\mathrm{Na}$ & $1.70 \mathrm{~m} \mathrm{~g} / \mathrm{L}$ & 0.10 \\
0.065 & $\mathrm{pH}$ & 5.81 & 0.19 \\
0.28 & $\mathrm{PO}$ as $\mathrm{P}$ & $0.008 \mathrm{~m} \mathrm{~g} / \mathrm{L}$ & 0.006 \\
0.015 & $\mathrm{SO}$ & $0.728 \mathrm{~m} \mathrm{~g} / \mathrm{L}$ & 0.390 \\
0.027 & SpCond & $17.0 \mu \mathrm{S} / \mathrm{cm}$ & 1.3 \\
0.006 & & & \\
\hline
\end{tabular}

$\mathrm{Hg}-18$ (mercury)

Analyte MPV F-pseudosigma

$\mathrm{Hg} \quad 0.70 \mu \mathrm{g} / \mathrm{L}$

0.12 